\title{
Strategy for the synthesis of pyridazine heterocycles and its derivatives
}

Hassen Bel Abed, ${ }^{\S}$ Oscar Mammoliti, ${ }^{\circledR}$ Omprakash Bande, ${ }^{\S}$ Guy Van Lommen ${ }^{\llbracket}$ and Piet Herdewijn ${ }^{\S}{ }^{*}$ ${ }^{\S}$ Rega Institute for Medical Research, Laboratory of Medicinal Chemistry, Katholieke Universiteit Leuven, Minderbroedersstraat 10, B-3000 Leuven, Belgium

${ }^{\top}$ Galapagos, Laboratory of Medicinal Chemistry, Generaal De Wittelaan L11 A3, B-2800 Mechelen, Belgium

*Corresponding author : e-mail : piet.herdewijn@,rega.kuleuven.be; Tel. : +32 (0)16/33.73.87 ; Fax : $+32(0) 16 / 33.73 .40$

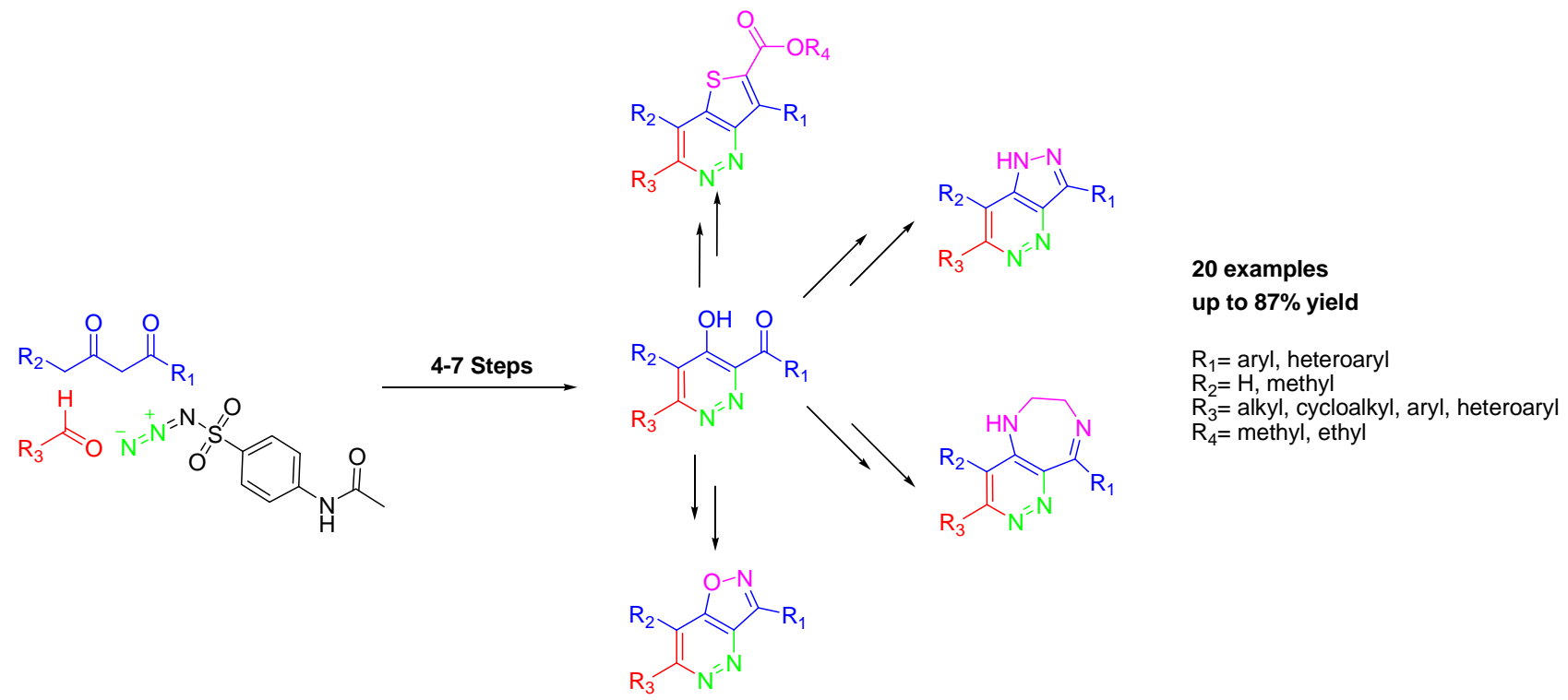

\begin{abstract}
The first synthesis of novel fused pyridazines has been realized starting from 1,3-diketones involving a Diaza-Wittig reaction as a key-step. A convenient strategy was elaborated to access versatile pyridazine derivatives allowing the variation of substituents at position 6 of the heterocyclic ring. In a first part, pyridazines bearing an ester group were synthesized as a model to evaluate the methodology. In a second part, an improved procedure has been used for the synthesis of pyridazines bearing a ketone group and different methods of cyclization were carried out, leading to several hitherto unknown biheterocyclic compounds. This reaction scheme represents an attractive methodology for the synthesis of novel fused pyridazine derivatives.
\end{abstract}




\section{Introduction}

Recently, pyridazines have been considered by GlaxoSmithKline as one of the "most developable" heteroaromatic rings for drug design. ${ }^{1}$ Pyridazine analogues proved to be useful ligands for different targets and have been proposed as "privileged structure" for drug discovery. ${ }^{2}$ Several compounds with pyridazine rings demonstrate biological activity (1-4) and many examples of pyridazine structures are naturally occurring. ${ }^{3}$ Pyridazines were recognized as selective GABA-A receptor antagonists, such as minaprine $1 .{ }^{4}$ Volonterio et al. developed the synthesis of pyridazine-based scaffolds such as $\mathbf{2}$ to target protein/protein interaction as $\alpha$-helix mimetics. ${ }^{5}$ 3-Amino-6-aryl-pyridazines have been considered as an interesting pharmacophore in drug discovery. Some compounds show biological activity ranging from obesity ${ }^{6}$ or neurodegenerative diseases ${ }^{7}$ to inflammatory pain such as the selective $\mathrm{CB}_{2}$ agonist 3 . $^{8}$ Among kinase inhibitors, different compounds containing the diazine pyridazine have been identified. For example, compound $\mathbf{4}$ is based on a pyridazinone ring and has been identified as a potent p38 MAP inhibitor $^{9}$ (Figure 1).

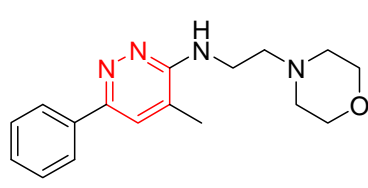
Antidepressant

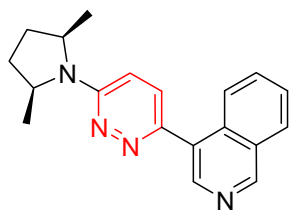

3

Selective $\mathrm{CB}_{2}$ agonist

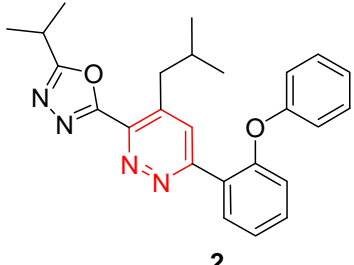

$\alpha$-Helix mimetics

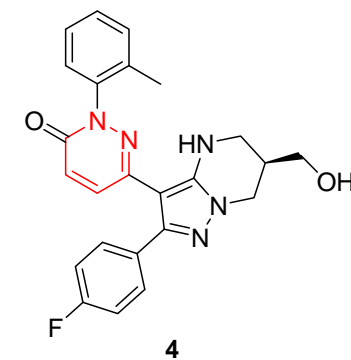

p38 MAP kinase inhibitor

Figure 1. Biologically active pyridazine derivatives.

The nitrogen containing heterocycle pyridazine is a key intermediate in the synthesis of several fused heterocycles used in drug discovery. ${ }^{10}$ Fused pyridazines can be distinguished into two classes. The first one having a bridgehead nitrogen atom. Some of them can be obtained from substituted 3amino-pyridazine which is easily available from substituted 3-chloro-pyridazine, and further cyclized to a bi-heterocyclic structure. Generally rings fused to pyridazines are 5-membered rings. Triazolo[4,3- 
b]pyridazine such as PIM-1 inhibitor $5^{11}$ can be considered as an example of this class of fused pyridazines. These derivatives were obtained after hydrazinolysis and cyclization of substituted 3chloro-pyridazines. Another example of this class of compounds is a VEGFR2 kinase inhibitor based on a imidazo[1,2-b]pyridazine 6 scaffold, ${ }^{12}$ which can be obtained by condensing substituted 3 -aminopyridazines with 2-chloro-acetaldehyde.

The second class represents pyridazines fused to a 6- or 5-membered ring, without a bridgehead nitrogen atom. Examples of this class of compounds are the phosphodiesterase $10 \mathrm{~A}$ inhibitors $7^{13}$ and the melanin-concentrating hormone 1 antagonist ${ }^{14} 8$ (Figure 2).

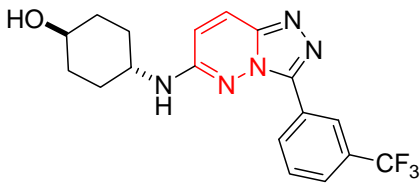

5 Pim-1 kinase inhibitor

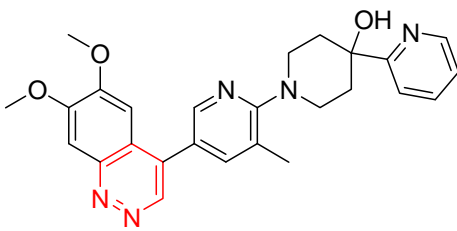

PDE10A inhibitor

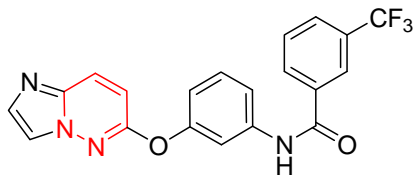

6

VEGFR2 kinase inhibitor

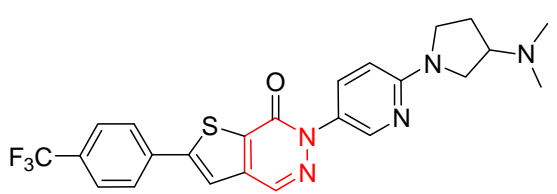

Melanin-concentrating hormone receptor 1 antagonist

Figure 2. Biologically active fused pyridazine derivatives.

As part of an ongoing project on the synthesis of small heterocycles of pharmaceutical interest, ${ }^{15} \mathrm{a}$ strategy for the synthesis of functionalized pyridazines by a Diaza-Wittig ${ }^{16}$ reaction has been developed. In search for operationally simple synthetic processes, we have now developed a convenient and safe method for the synthesis of 6-substituted-4-hydroxy-3-methoxycarbonyl pyridazines starting from methyl acetoacetate. We described herein the synthesis of a whole series of existing and new biheterocyclic scaffolds such as pyrazolo[4,3-c]pyridazines, thieno[3,2-c]pyridazines, isoxazolo[4,5c]pyridazines and $5 \mathrm{H}, 6 \mathrm{H}, 7 \mathrm{H}$-pyridazino[4,3-e][1,4]diazepine starting from $\alpha$-diazo-1,3-diketone precursors. 


\section{Results and discussion}

In order to explore novel heterocycles for drug design projects, we became interested in developing convenient methods for the synthesis of the pyridazine ring. ${ }^{17}$ The synthesis of 6 -substituted-4hydroxy-3-methoxycarbonyl pyridazine derivatives was first envisioned as starting material. This compound can be obtained by the method described by Zalesov et al. using furan-4,5-dione as an intermediate. ${ }^{18}$ However, this method has several limitations and only aryl substituted pyridazines are accessible. A method allowing the introduction of various substituents at the position 6 such as alkyls, cycloalkyls, aryls and heteroaryls could be more valuable. Moreover, a strategy which allows modifications in a site-selective way could be appealing. The starting material chosen is the readily available methyl acetoacetate 9 which has been subjected to a diazo transfer reaction ${ }^{19}$ with $p$ acetamido benzene sulfonyl azide ( $p$-ABSA) in acetonitrile for 2 hours at room temperature to obtain the $\alpha$-diazo- $\beta$-ketoester 10 in good yields. Following the titanium aldol method developed by the Calter group ${ }^{20}$ the diazo derivative $\mathbf{1 0}$ was converted to the aldol $\mathbf{1 1}$ in moderate to good yields. Mild oxidation of 11 with IBX (1-hydroxy-1,2-benziodoxol-3(1H)-one 1-oxide) ${ }^{21}$ led to the $\alpha$-diazo- $\beta$ ketoester which was used without further purification. The final step to obtain the pyridazine derivatives is the Diaza-Wittig reaction. Formation of the phosphazine intermediate and the subsequent Diaza-Wittig reaction occur as a tandem process with HMPT (hexamethyl phosphorus triamide) in dichloromethane at room temperature for 16 hours to afford the pyridazine $\mathbf{1 2}$ in good yields (Scheme $1)$.

Scheme 1. Synthesis of 6-substituted-4-hydroxy-3-methoxycarbonyl pyridazines.

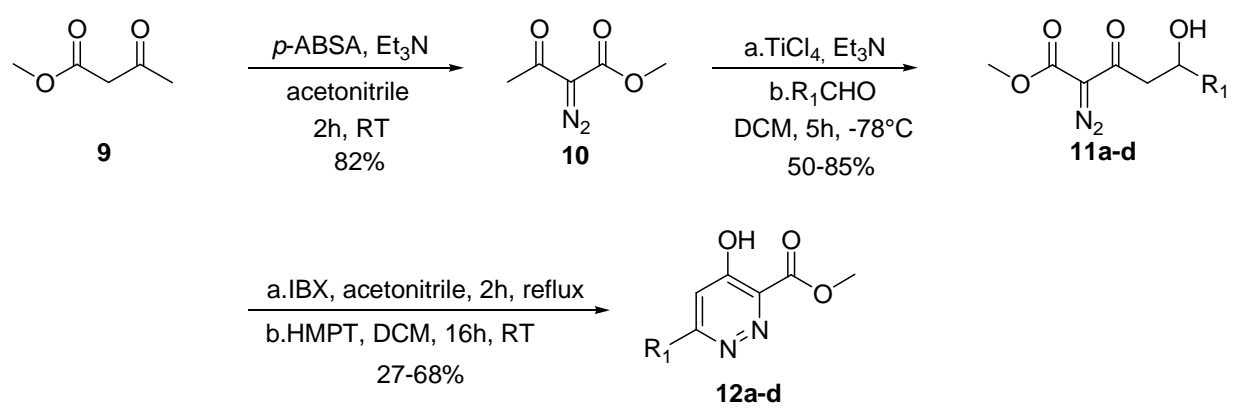

Due to the toxicity ${ }^{22}$ of HMPT used for the Diaza-Wittig reaction, an improvement of the method to synthesize pyridazines has been investigated. Different phosphines have been screened to convert the derivative 11c into the pyridazine 12c after oxidation with IBX. A first attempt to replace HMPT by triphenyl phosphine was not successful. In fact, after two days of reaction in diethyl ether at room temperature, no $\alpha$-diazo- $\beta$-ketoester was converted into the corresponding pyridazine (Table 1 , entry 
2). An increase of the temperature to $60^{\circ} \mathrm{C}$ by using tetrahydrofuran as a solvent led to the same result (Table 1, entry 3). By using triethyl phosphite, the pyridazine was obtained in a lower yield than with HMPT (Table 1, entry 4). Triethyl phosphine allowed the formation of the pyridazine in 5 hours in dichloromethane (Table 1, entry 5). Due to the toxicity and facile oxidation of triethyl phosphine,$^{23}$ the tributyl phosphine was explored and the pyridazine was obtained in less than 1 hour at room temperature (Table 1, entry 6) in good yields. Diisopropyl ether $\left(i-\operatorname{Pr}_{2} \mathrm{O}\right)$ proved to be the best solvent for this reaction, allowing to obtain the pyridazine 12c as precipitate in less than 30 minutes in high yields (Table 1, entry 8). This modification represents an improved and safe method for the synthesis of the pyridazine derivatives 12 .

Table 1. Influence of phosphines and solvents on the Diaza-Wittig reaction. ${ }^{a}$

\begin{tabular}{|c|c|c|c|c|}
\hline entry & phosphine & solvent & conditions & yield of $12 \mathrm{c}(\%)^{b}$ \\
\hline 1 & HMPT & $\mathrm{CH}_{2} \mathrm{Cl}_{2}$ & $16 \mathrm{~h}, \mathrm{RT}$ & $60^{c}$ \\
\hline 2 & $\mathrm{P}(\mathrm{Ph})_{3}$ & $\mathrm{Et}_{2} \mathrm{O}$ & 48 h, RT & $-d$ \\
\hline 3 & $\mathrm{P}(\mathrm{Ph})_{3}$ & THF & $16 \mathrm{~h}, 60^{\circ} \mathrm{C}$ & $-d$ \\
\hline 4 & $\mathrm{P}(\mathrm{OEt})_{3}$ & $\mathrm{CH}_{2} \mathrm{Cl}_{2}$ & 16 h, RT & $37^{c}$ \\
\hline 5 & $\mathrm{P}(\mathrm{Et})_{3}$ & $\mathrm{CH}_{2} \mathrm{Cl}_{2}$ & $5 \mathrm{~h}, \mathrm{RT}$ & $52^{c}$ \\
\hline 6 & $\mathrm{P}(n-\mathrm{Bu})_{3}$ & $\mathrm{CH}_{2} \mathrm{Cl}_{2}$ & $1 \mathrm{~h}, \mathrm{RT}$ & $58^{c}$ \\
\hline 7 & $\mathrm{P}(n-\mathrm{Bu})_{3}$ & $\mathrm{Et}_{2} \mathrm{O}$ & $30 \mathrm{~min}, \mathrm{RT}$ & $53^{c}$ \\
\hline 8 & $\mathbf{P}(n-\mathbf{B u})_{3}$ & $i-\operatorname{Pr}_{2} \mathrm{O}$ & $30 \mathrm{~min}, \mathrm{RT}$ & $70^{e}$ \\
\hline
\end{tabular}

${ }^{a}$ All reactions were performed using $1 \mathrm{mmol}$ of 11c and $364 \mathrm{mg}$ of IBX in refluxing acetonitrile for 2 hours, followed by a filtration to obtain the $\alpha$-diazo- $\beta$-ketoester. The crude compound was stirred with $183 \mu \mathrm{L}$ of phosphine following the conditions described above. ${ }^{b}$ Isolated yield. ${ }^{c}$ Purification by flash column chromatography on silica gel. ${ }^{d} \alpha$-diazo- $\beta$ ketoester recovered. ${ }^{e}$ Precipitation of 12c.

The selection of appropriate aldehydes $\left(\mathrm{R}_{1} \mathrm{CHO}\right.$, scheme 1$)$ allows the synthesis of pyridazine with different substituents at position 6 such as alkyl (Table 2, entry 1), cycloalkyl (Table 2, entry 2), aryl (Table 2, entry 3) and heteroaryl (Table 2, entry 4). The yield of the Diaza-Wittig reaction was higher with alkyl and cycloalkyl groups than aryl and heteroaryl groups. Invariable, the use of $\mathrm{P}(n-\mathrm{Bu})_{3}$ gives higher yields of pyridazines than while using HMPT. Some of the derivatives were obtained as precipitate (Table 2, entry 1-3) in less than 30 minutes of reaction. 
Table 2. Synthesis of 6-substituted-4-hydroxy-3-methoxycarbonyl pyridazines. ${ }^{a}$

aldehyde
derivative

${ }^{a}$ All reactions were performed using $1 \mathrm{mmol}$ of $\mathbf{1 1}$ and $364 \mathrm{mg}$ of IBX in refluxing acetonitrile for 2 hours, followed by a filtration to obtain the corresponding $\alpha$-diazo- $\beta$-ketoester. The crude compound was stirred with $183 \mu \mathrm{L}$ of HMPT in dichloromethane for 16 hours (HMPT method) or with $250 \mu \mathrm{L}$ of $\mathrm{P}(n-\mathrm{Bu})_{3}$ in $i-\mathrm{Pr}_{2} \mathrm{O}$ for 30 minutes $\left(\mathrm{P}(n-\mathrm{Bu})_{3}\right.$ method). ${ }^{b}$ Isolated yield. ${ }^{c}$ Purification by flash column chromatography on silica gel. ${ }^{d}$ Precipitation of $\mathbf{1 2 .}$

For the building of pyridazines bearing a 3-keto group, starting material of type $\mathbf{1 3}$ is needed. This approach will, likewise, allow us to obtain pyridazine derivatives, useful for the formation of new bicyclic heterocycles.

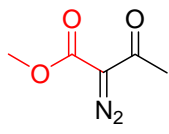

10
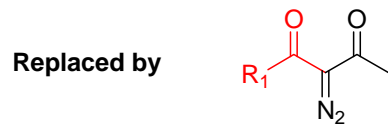

13

$\mathrm{R}_{1}=$ aryl, heteroaryl

Figure 3. Diazo derivatives.

The reaction scheme starts with the synthesis of $\beta$-hydroxy ketone. In order to avoid any side reaction during the synthetic route, $\mathrm{R}_{1}$ has been chosen to be an aryl or heteroaryl group (Figure 3 ). Different methods for the synthesis of 1,3-diketone have been described in the past. ${ }^{24} \mathrm{We}$ have followed two different approaches. The first one is based on L-proline organocatalysis discovered by List et al.. ${ }^{25}$ This strategy was applied on aldehydes bearing an electron withdrawing group (nitro group 
and cyano group) 14 to afford $\beta$-hydroxy ketone 15. The final $\alpha$-diazo-1,3-diketone 16 was obtained by mild oxidation with IBX, followed by a diazo transfer reaction with $p$-ABSA. However, when using other aldehydes, the reaction becomes slow and at least one day of reaction is required to afford the corresponding $\beta$-hydroxy ketone. ${ }^{26}$

Scheme 2. Synthesis of $\alpha$-diazo-1,3-diketone.

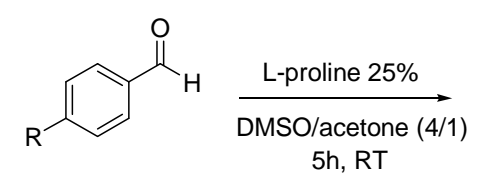

14a, $\mathrm{R}=\mathrm{NO}_{2} \quad \mathrm{R}=\mathrm{NO}_{2}, 57 \%$ 14b, R=CN $\quad \mathrm{R}=\mathrm{CN}, 55 \%$

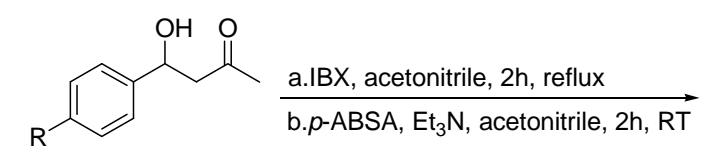

15a, $\mathrm{R}=\mathrm{NO}_{2}$ $15 \mathrm{a}, \mathrm{R}=\mathrm{NO}_{2}$
$15 \mathrm{~b}, \mathrm{R}=\mathrm{CN}$
$=\mathrm{NO}_{2}, 85 \%$ $\mathrm{R}=\mathrm{CN}, 77 \%$<smiles>[R]c1ccc(C(=O)C(C)=O)cc1</smiles>

$16 \mathrm{a}, \mathrm{R}=\mathrm{NO}_{2}$ $16 \mathrm{~b}, \mathrm{R}=\mathrm{CN}$

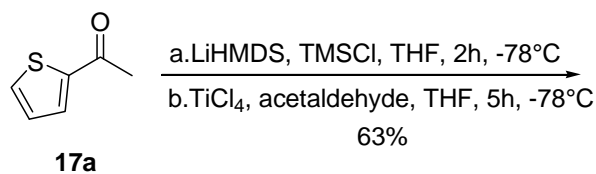

$17 a$

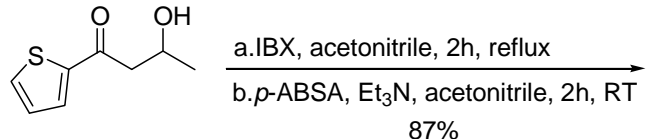

$18 \mathrm{a}$

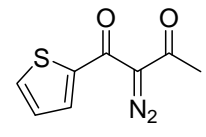

$19 \mathrm{a}$<smiles>CCC(=O)C(=N)C(=O)c1ccc(Cl)cc1</smiles>
$17 \mathrm{~b}$

$68 \%$

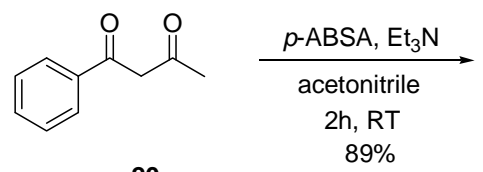

20<smiles>CC(=O)C(=N)C(=O)c1ccccc1</smiles>

21

Therefore, for the other $\alpha$-diazo-1,3-diketone derivatives, a strategy based on the Mukaiyama aldol reaction ${ }^{27}$ has been used. The acetophenone derivative 17 was converted to the corresponding silyl enol ether at $-78{ }^{\circ} \mathrm{C}$ with LiHMDS and trimethylsilyl chloride. The silyl enol ether from 17 reacted with acetaldehyde or propionaldehyde in the presence of titanium tetrachloride to afford 18. The desired diazo compound 19 was obtained after oxidation with IBX and diazo transfer reaction with $p$-ABSA.

A commercially available reagent $\mathbf{2 0}$ was used to synthesize the $\alpha$-diazo-1,3-diketone $\mathbf{2 1}$ by a Regitz reaction with $p$-ABSA. Five different $\alpha$-diazo-1,3-diketones 16a, 16b, 19a, 19b and 21 became available in this way.

The $\alpha$-diazo-1,3-diketone derivatives were used for the synthesis of pyridazines. The first step was the titanium aldol reaction to afford the $\beta$-hydroxy- $\alpha$-diazo diketone 22 . It should be noted that these aldol products 22 are easily degraded (water elimination) ${ }^{28}$ to give the corresponding enone 23 
(complete degradation was observed during purification by flash column chromatography on silica gel).

Derivative 22 was oxidized with IBX in refluxing acetonitrile. The crude product was used without further purification in the Diaza-Wittig reaction. Treatment with tributyl phosphine in diisopropyl ether for 30 minutes led to the desired pyridazine 24 bearing a ketone group at position 3 .

Scheme 3. Synthesis of pyridazine derivatives bearing a ketone group.

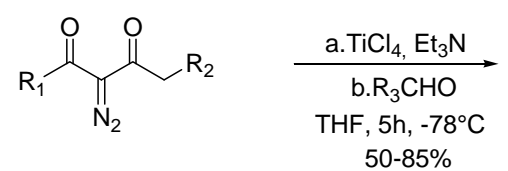

$16 a, 16 b, 19 a, 19 b, 21$<smiles>[R]C(=O)C(=N)C(=O)C([R2])C([R3])O</smiles>

22b-j<smiles>[R]C(=O)c1nnc([R])c(O)c1[R]</smiles>

24b-j

$\mathrm{R}_{3}=$ alkyl, cycloalkyl, aryl, heteroaryl

Table 3. Synthesis of 6-substituted-4-hydroxy pyridazines bearing a ketone group on position $3 .^{a}$

entry


7<smiles>CC(=O)C(C)=NC(=O)c1ccccc1</smiles><smiles>CC(=O)C(=N)C(=O)c1ccccc1</smiles><smiles>CC(=O)C(=N)C(=O)c1ccccc1</smiles>

21<smiles>O=Cc1ccccc1</smiles>

21<smiles>O=CC1CCCCC1</smiles>

21<smiles>O=C(c1ccccc1)c1nnc(-c2ccccc2)cc1O</smiles>

24h $48^{d}$<smiles>O=C(c1ccccc1)c1nnc(C2CCCCC2)cc1O</smiles>

$24 i$ $52^{d}$<smiles>CC(C)c1cc(O)c(C(=O)c2ccccc2)nn1</smiles>

${ }^{a}$ All reactions were performed using $1 \mathrm{mmol}$ of 22 and $364 \mathrm{mg}$ of IBX in refluxing acetonitrile for $2 \mathrm{~h}$, followed by a filtration to obtain the corresponding $\alpha$-diazo- $\beta$-ketoester. The crude compound was stirred with $250 \mu \mathrm{L}$ of $\mathrm{P}(n-\mathrm{Bu})_{3}$ in $i$ $\mathrm{Pr}_{2} \mathrm{O}$ for $30 \mathrm{~min}$ at room temperature. ${ }^{b}$ Isolated yield. ${ }^{\text {P Precipitation of } 24 .}{ }^{d}$ Purification by flash column chromatography on silica gel.

This strategy led to the synthesis of nine novel pyridazine derivatives covering different substitutions, such as several aryl ketones (Table 3, entry 1-3, 5-9) and a heteroaryl ketone (Table 3, entry 4). On the pyridazine ring, it was possible to introduce various $\mathrm{R}_{3}$ groups such as alkyl (Table 3, entry $1,2,4,5,9$ ), cycloalkyl (Table 3, entry 8), aryl (Table 3, entry 3, 7) and heteroaryl (Table 3, entry 6). The use of 19b allowed the synthesis of a tetrasubstituted pyridazine (Table 3, entry 5) with $\mathrm{R}_{2}$ being a methyl group. The pyridazine analogues $\mathbf{2 4 b}$-j have been subjected to different cyclization methods to obtain various fused pyridazines. 
Scheme 4. Synthesis of fused pyridazines by 4 different methods (A, B, C, D).

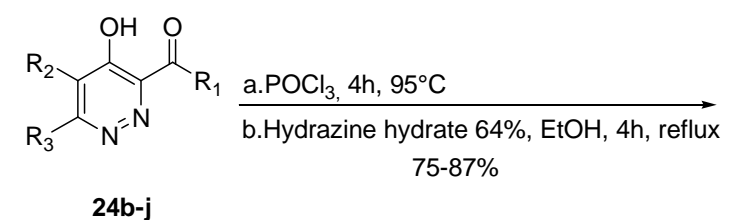<smiles>[R]c1nnc2c([R])n[nH]c2c1[R]</smiles>

25a-f

Method A
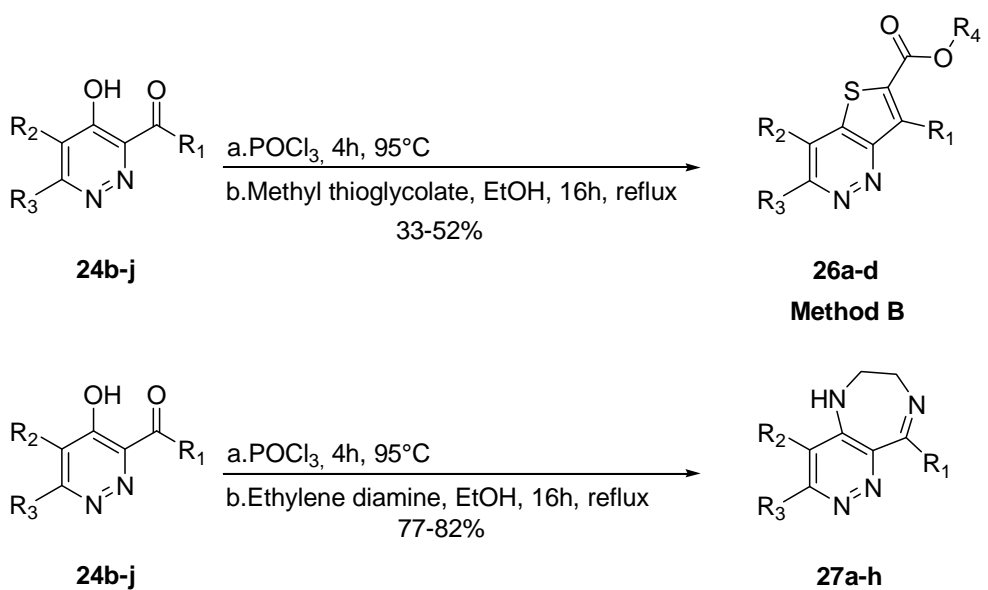

27a-h

Method C

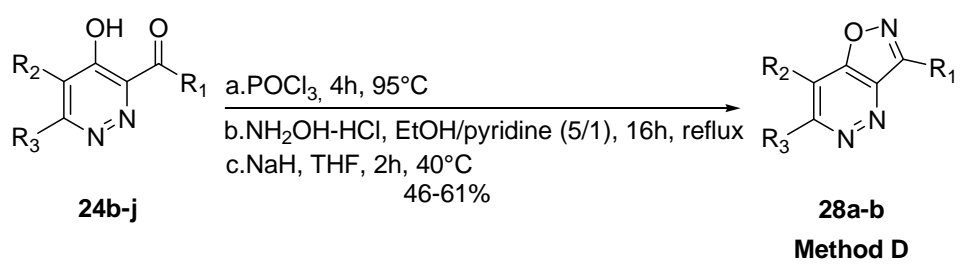

$$
\begin{aligned}
& \mathrm{R}_{1}=\text { aryl, heteroaryl } \\
& \mathrm{R}_{2}=\mathrm{H} \text {, methyl } \\
& \mathrm{R}_{3}=\text { alkyl, cycloalkyl, aryl, heteroaryl } \\
& \mathrm{R}_{4}=\text { methyl, ethyl }
\end{aligned}
$$

The first scaffold investigated was the pyrazolo[4,3-c]pyridazine 25. After chlorination of $\mathbf{2 4}$ with phosphorous oxychloride, the crude product was refluxed with hydrazine hydrate in ethanol for 4 hours to lead to the desired product 25 (method A). A condensation of the methyl thioglycolate on the chlorinated pyridazine in refluxing ethanol for 16 hours led to the thieno[3,2-c]pyridazine 26 (method B), only 26d was containing a methyl ester group, the other derivatives synthesized (26a, 26b and 26c) were bearing an ethyl ester group due to possible transesterification with ethanol during the reaction.

In our search for novel scaffolds, we described the first synthesis of 5H,6H,7H-pyridazino[4,3e] $[1,4]$ diazepine 27. Treatment of $\mathbf{2 4}$ with $\mathrm{POCl}_{3}$, followed by a cyclization with ethylene diamine in refluxing ethanol for 16 hours, led to the fused pyridazine 27 (method C). Another novel scaffold that was synthesized with this strategy, is isoxazolo[4,5-c]pyridazine 28. The ketone of the chlorinated 
pyridazine was converted into an oxime with hydroxylamine hydrochloride and finally cyclized by heating at $40{ }^{\circ} \mathrm{C}$ for 2 hours with sodium hydride in tetrahydrofuran to afford $\mathbf{2 8}$ (method D).

Table 4. Synthesis of fused pyridazine analogues.

\begin{tabular}{|c|c|c|c|c|c|c|}
\hline entry & pyridazine & & method & fused & & yield $(\%)^{a}$ \\
\hline 1 & & $24 b$ & $\mathrm{~B}^{b}$ & & 26a & 52 \\
\hline 2 & & $24 b$ & $\mathrm{C}^{c}$ & & $27 a$ & 77 \\
\hline 3 & & $24 c$ & $\mathrm{~A}^{d}$ & & $25 a$ & 83 \\
\hline 4 & & 24d & $\mathrm{A}^{d}$ & & $25 b$ & 79 \\
\hline 5 & & 24d & $\mathrm{C}^{c}$ & & 27b & 79 \\
\hline 6 & & $24 \mathrm{e}$ & $\mathrm{B}^{b}$ & & $26 b$ & 38 \\
\hline 7 & & $24 e$ & $\mathrm{C}^{c}$ & & $27 c$ & 81 \\
\hline 8 & & $24 f$ & $\mathrm{~A}^{d}$ & & $25 c$ & 87 \\
\hline 9 & & $24 f$ & $\mathrm{C}^{c}$ & & $27 d$ & 82 \\
\hline
\end{tabular}


10<smiles>CCc1nnc(C(=O)c2ccc(Cl)cc2)c(O)c1C</smiles>

$24 f$

$\mathrm{D}^{e}$

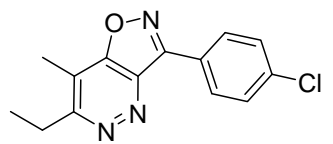

$28 a$

61

11<smiles>O=C(c1ccccc1)c1nnc(-c2cccs2)cc1O</smiles>

24g $\quad \mathrm{A}^{d}$

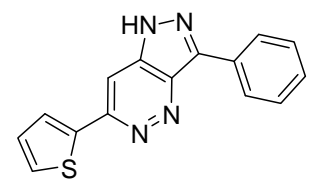

25d

81

12<smiles>O=C(c1ccccc1)c1nnc(-c2cccs2)cc1O</smiles>

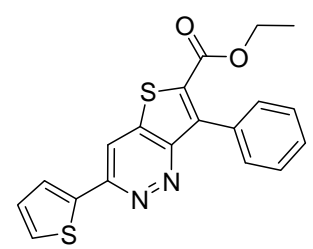

26c

33

13<smiles>O=C(c1ccccc1)c1nnc(-c2cccs2)cc1O</smiles>

24g

$\mathrm{C}^{c}$<smiles>c1ccc(C2=NCCNc3cc(-c4cccs4)nnc32)cc1</smiles>

27e

79

14<smiles>O=C(c1ccccc1)c1nnc(-c2cccs2)cc1O</smiles><smiles>c1ccc(-c2noc3cc(-c4cccs4)nnc23)cc1</smiles>

28b

46

15<smiles>O=C(c1ccccc1)c1nnc(-c2ccccc2)cc1O</smiles><smiles>c1ccc(-c2cc3[nH]nc(-c4ccccc4)c3nn2)cc1</smiles>

25e

79

16<smiles>O=C(c1ccccc1)c1nnc(-c2ccccc2)cc1O</smiles>

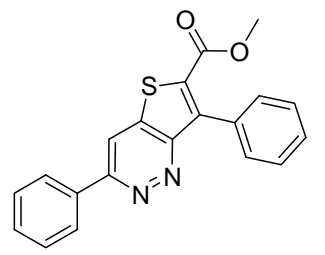

26d

41

17<smiles>O=C(c1ccccc1)c1nnc(-c2ccccc2)cc1O</smiles><smiles>c1ccc(C2=NCCNc3cc(-c4ccccc4)nnc32)cc1</smiles>

27f

77

18<smiles>O=C(c1ccccc1)c1nnc(C2CCCCC2)cc1O</smiles>

$24 i$

$\mathrm{C}^{c}$<smiles>c1ccc(C2=NCCNc3cc(C4CCCCC4)nnc32)cc1</smiles>

$27 g$

79

19<smiles>CC(C)c1cc(O)c(C(=O)c2ccccc2)nn1</smiles>

24j

$\mathrm{A}^{d}$

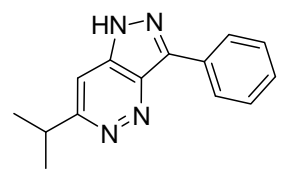

$25 f$

75

12 


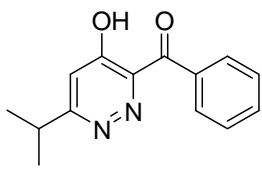

${ }^{a}$ Isolated yield. ${ }^{b}$ Synthesis of thieno[3,2-c]pyridazine 26 with method B, see the experimental section for details. ${ }^{c}$ Synthesis of $5 \mathrm{H}, 6 \mathrm{H}, 7 \mathrm{H}$-pyridazino[4,3-e][1,4]diazepine 27 with method $\mathrm{C}$, see the experimental section for details. ${ }^{d}$ Synthesis of pyrazolo[4,3-c]pyridazine 25 with method A, see the experimental section for details. ${ }^{e}$ Synthesis of isoxazolo[4,5c]pyridazine 28 with method D, see the experimental section for details.

\section{Conclusion}

In conclusion, we have developed a synthetic scheme for the synthesis of pyridazine derivatives bearing an ester group 12 starting from methyl acetoacetate 9. The method has been improved by replacing HMPT by $\mathrm{P}(n-\mathrm{Bu})_{3}$ to perform the Diaza-Wittig reaction in a shorter reaction time and with better yields. Based on this methodology and starting from 1,3-diketones 13, it was possible to synthesize novel pyridazines bearing a ketone group 24 with different substituents at position 6 . These pyridazine analogues were used for the development of a 20-member library of novel biheterocyclic compounds such as pyrazolo[4,3-c]pyridazine 25 and thieno[3,2-c]pyridazine 26. It is also the first report dealing with the synthesis of $5 \mathrm{H}, 6 \mathrm{H}, 7 \mathrm{H}$-pyridazino[4,3-e][1,4]diazepine 27 and isoxazolo[4,5c]pyridazine 28. 


\section{Experimental section}

For all reactions, analytical grade solvents were used. All moisture-sensitive reactions were carried out in oven-dried glassware $\left(135^{\circ} \mathrm{C}\right)$ under a nitrogen or argon atmosphere. Reaction temperatures are reported as bath temperature. Precoated aluminum sheets were used for TLC. Compounds were visualized with UV light $(\lambda=254 \mathrm{~nm})$. Products were purified by flash chromatography on silica gel 63-200, 60 A. Melting points were obtained on a melting point apparatus with open capillary tubes. ${ }^{1} \mathrm{H}$ and ${ }^{13} \mathrm{C}$ NMR spectra were recorded on $300 \mathrm{MHz}, 500 \mathrm{MHz}$ and $600 \mathrm{MHz}$ spectrometer using $\mathrm{CDCl}_{3}$ and DMSO- $\mathrm{d}_{6}$ as the solvent. The ${ }^{1} \mathrm{H}$ and ${ }^{13} \mathrm{C}$ chemical shifts were referenced to residual solvent signals at $\delta{ }^{1} \mathrm{H} /{ }^{13} \mathrm{C} 7.26 / 77.00\left(\mathrm{CDCl}_{3}\right)$ and 2.50/39.50 (DMSO-d6) relative to TMS as internal standard. Coupling constants $J[\mathrm{~Hz}]$ were directly taken from the spectra. Splitting patterns are designated as $\mathrm{s}$ (singlet), d (doublet), t (triplet), q (quartet), m (multiplet) and br (broad). High resolution mass spectra were acquired on a quadrupole orthogonal acceleration time-of-flight mass spectrometer. Samples were infused at $3 \mu \mathrm{L} / \mathrm{min}$ and spectra were obtained in positive (or negative) ionization mode with a resolution of 15000 (FWHM) using leucine enkephalin as lock mass. Electrospray MS spectra were obtained on a LC/MS spectrometer. All the LC/MS analysis methods are using $\mathrm{MeCN} / \mathrm{H}_{2} \mathrm{O}$ gradients. Water contains either $0.1 \%$ TFA or $0.1 \% \mathrm{NH}_{3}$.

\section{Methyl 2-diazo-3-oxobutanoate (10)}

To a solution of compound 9 ( $5 \mathrm{~g}, 43.06 \mathrm{mmol})$ in $30 \mathrm{~mL}$ of acetonitrile under argon at $0{ }^{\circ} \mathrm{C}$ was added successively triethylamine $(7.8 \mathrm{~mL}, 55.98 \mathrm{mmol})$ and $p$-acetamido benzene sulfonyl azide ( $p$-ABSA) (10.4 g, $43.06 \mathrm{mmol}$ ). The mixture was stirred for 2 hours and was allowed to warm to room temperature and was diluted with $100 \mathrm{~mL}$ of $\mathrm{Et}_{2} \mathrm{O} / n$-Hexane $(1: 1)$ and then filtered. The filtrate was concentrated and the residue purified by flash column chromatography on silica gel (EtOAc/n-Hexane $=1: 2)$ to afford the desired compound $\mathbf{1 0}(5 \mathrm{~g}, 35.18 \mathrm{mmol}, 82 \%$ yield $)$ as yellow oil. Data for $\mathbf{1 0}:{ }^{1} \mathrm{H}$ NMR (500 MHz, DMSO-d6) $\delta 3.65$ (s, 3H), 2.18 (s, 3H); ${ }^{13} \mathrm{C}$ NMR (125 MHz, DMSO-d6) $\delta 189.3$, $161.5,52.3,27.9$.

\section{General procedure for the preparation of 11a-d by titanium aldol reaction:}

To a solution of $10(1 \mathrm{~g}, 7.04 \mathrm{mmol})$ in $50 \mathrm{~mL}$ of $\mathrm{CH}_{2} \mathrm{Cl}_{2}$ under argon at $-78{ }^{\circ} \mathrm{C}$ was added dropwise $\mathrm{TiCl}_{4}(849 \mu \mathrm{L}, 7.74 \mathrm{mmol})$ followed by $\mathrm{Et}_{3} \mathrm{~N}$ (1.08 $\left.\mathrm{mL}, 7.74 \mathrm{mmol}\right)$. The resulting red solution was stirred at $-78{ }^{\circ} \mathrm{C}$ for 1 hour, after which a solution of aldehyde $(6.34 \mathrm{mmol})$ in $\mathrm{CH}_{2} \mathrm{Cl}_{2}$ was slowly added. The reaction mixture was stirred at $-78{ }^{\circ} \mathrm{C}$ for 4 hours and then the reaction was quenched with 
$50 \mathrm{~mL}$ of saturated aqueous $\mathrm{NH}_{4} \mathrm{Cl}$ and warmed to room temperature. The organic layer was separated and then washed with $40 \mathrm{~mL}$ of saturated aqueous $\mathrm{NaHCO}_{3}$. The aqueous layers were extracted with 50 $\mathrm{mL}$ of $\mathrm{CH}_{2} \mathrm{Cl}_{2}$. The combined organic layers were dried over $\mathrm{Na}_{2} \mathrm{SO}_{4}$ and concentrated in vacuo. The product was purified by flash column chromatography on silica gel (EtOAc/n-Hexane = 1:3) to afford the aldol product 11.

\section{Methyl 2-diazo-5-hydroxy-3-oxoheptanoate (11a)}

Yellow oil, 1.2 g (85\% yield); Synthesized from propionaldehyde. Spectral and analytical data were in agreement with previous reports. ${ }^{15(b)}$

\section{Methyl 5-cyclohexyl-2-diazo-5-hydroxy-3-oxopentanoate (11b)}

Yellow oil, 1.3 g (73\% yield); Synthesized from cyclohexane carboxaldehyde. Spectral and analytical data were in agreement with previous reports. ${ }^{15(\mathrm{~b})}$

\section{Methyl 2-diazo-5-hydroxy-3-oxo-5-phenylpentanoate (11c)}

Yellow oil, 1.3 g (75\% yield); Synthesized from benzaldehyde. Spectral and analytical data were in agreement with previous reports. ${ }^{15(b)}$

\section{Methyl 2-diazo-5-(furan-2-yl)-5-hydroxy-3-oxopentanoate (11d)}

Yellow oil, $840 \mathrm{mg}$ (50\% yield); Synthesized from 2-furfural. Spectral and analytical data were in agreement with previous reports. ${ }^{15(b)}$

\section{HMPT method: preparation of pyridazine derivatives 12a-d:}

To a solution of 11 ( $1 \mathrm{mmol})$ in $10 \mathrm{~mL}$ of acetonitrile under argon was added IBX (1-hydroxy-1,2benziodoxol-3(1H)-one 1-oxide) (364 mg, $1.3 \mathrm{mmol})$. The mixture was refluxed for 2 hours and was allowed to warm to room temperature and then filtered. The filtrate was concentrated to afford the $\alpha$ diazo- $\beta$-ketoester used directly without further purification for the next step.

To a solution of $\alpha$-diazo- $\beta$-ketoester in $10 \mathrm{~mL}$ of $\mathrm{CH}_{2} \mathrm{Cl}_{2}$ was added HMPT (hexamethylphosphorous triamide) $(183 \mu \mathrm{L}, 1 \mathrm{mmol})$. The reaction mixture was stirred under argon at room temperature for 16 hours. After completion of the reaction (monitored by TLC), the reaction mixture was quenched with the addition of water. The organic layer was washed with water $(2 \times 10 \mathrm{~mL})$, dried over $\mathrm{Na}_{2} \mathrm{SO}_{4}$ and 
concentrated in vacuo. A purification by flash column chromatography on silica gel (EtOAc/n-Hexane =9:1) afforded the desired pyridazine 12 .

\section{$\mathbf{P}(n-B u)_{3}$ method: preparation of pyridazine derivatives 12a-d:}

To a solution of 11 ( $1 \mathrm{mmol})$ in $10 \mathrm{~mL}$ of acetonitrile under argon was added IBX (1-hydroxy-1,2benziodoxol-3(1H)-one 1-oxide) (364 mg, $1.3 \mathrm{mmol})$. The mixture was refluxed for 2 hours and was allowed to warm to room temperature and then filtered. The filtrate was concentrated to afford the $\alpha$ diazo- $\beta$-ketoester used directly without further purification for the next step.

To a solution of $\alpha$-diazo- $\beta$-ketoester in $20 \mathrm{~mL}$ of $i-\operatorname{Pr}_{2} \mathrm{O}$ was added $\mathrm{P}(n-\mathrm{Bu})_{3}(250 \mu \mathrm{L}, 1 \mathrm{mmol})$. The reaction mixture was stirred under argon at room temperature for 30 minutes, which after this time a precipitate was formed. The suspension was filtered, washed with $i-\operatorname{Pr}_{2} \mathrm{O}$ and dried to afford 12 .

\section{Methyl 6-ethyl-4-hydroxypyridazine-3-carboxylate (12a)}

White solid, $124 \mathrm{mg}$ (68\% yield with HMPT method) and $155 \mathrm{mg}\left(85 \%\right.$ yield with $\mathrm{P}(n-\mathrm{Bu})_{3}$ method); mp 179-182 ${ }^{\circ} \mathrm{C}$. Spectral and analytical data were in agreement with previous reports. ${ }^{15(b)}$

\section{Methyl 6-cyclohexyl-4-hydroxypyridazine-3-carboxylate (12b)}

Beige solid, $146 \mathrm{mg}$ (62\% yield with HMPT method) and $170 \mathrm{mg}\left(72 \%\right.$ yield with $\mathrm{P}(n-\mathrm{Bu})_{3}$ method); $\mathrm{mp} 215-217^{\circ} \mathrm{C}$. Spectral and analytical data were in agreement with previous reports. ${ }^{15(b)}$

\section{Methyl 4-hydroxy-6-phenylpyridazine-3-carboxylate (12c)}

Pale yellow solid, $138 \mathrm{mg}$ (60\% yield with HMPT method) and $161 \mathrm{mg}$ (70\% yield with $\mathrm{P}(n-\mathrm{Bu})_{3}$ method); $\mathrm{mp} 211-212^{\circ} \mathrm{C}$. Spectral and analytical data were in agreement with previous reports..$^{15(b)}$

\section{Methyl 6-(furan-2-yl)-4-hydroxypyridazine-3-carboxylate (12d)}

Brown solid, $59 \mathrm{mg}$ (27\% yield with HMPT method) and $77 \mathrm{mg}$ (35\% yield with $\mathrm{P}(\mathrm{n}-\mathrm{Bu})_{3}$ method, a purification by flash column chromatography on silica gel (EtOAc/n-Hexane $=9: 1$ ) afforded the desired pyridazine 12d); mp 192-195 ${ }^{\circ} \mathrm{C}$. Spectral and analytical data were in agreement with previous reports. ${ }^{15(b)}$ 


\section{General procedure for the preparation of $\beta$-hydroxy ketone derivatives 15a-b by organocatalysis:}

To a solution of aldehyde $14(1 \mathrm{mmol})$ in a mixture of DMSO $(4 \mathrm{~mL})$ and acetone $(1 \mathrm{~mL})$ was added Lproline $(29 \mathrm{mg}, 0.25 \mathrm{mmol})$. The resulting mixture was stirred under argon at room temperature for 5 hours. The reaction mixture was treated with $10 \mathrm{~mL}$ of saturated aqueous $\mathrm{NH}_{4} \mathrm{Cl}$, the layers were separated, and the aqueous layer was extracted several times with EtOAc, dried over $\mathrm{Na}_{2} \mathrm{SO}_{4}$, and concentrated in vacuo. A purification by flash column chromatography on silica gel (EtOAc/n-Hexane $=1: 2$ ) afforded the desired aldol product 15.

\section{4-Hydroxy-4-(4-nitrophenyl)butan-2-one (15a)}

Pink solid, $119 \mathrm{mg}$ (57\% yield); mp 65-68 ${ }^{\circ} \mathrm{C}$; Data for 15a: ${ }^{1} \mathrm{H}$ NMR (500 MHz, DMSO-d6) $\delta 8.19(\mathrm{~d}$, $J=8.7 \mathrm{~Hz}, 2 \mathrm{H}), 7.64(\mathrm{~d}, J=8.7 \mathrm{~Hz}, 2 \mathrm{H}), 5.70(\mathrm{~d}, J=4.8 \mathrm{~Hz}, 1 \mathrm{H}), 5.13-5.16(\mathrm{~m}, 1 \mathrm{H}), 2.75(\mathrm{~d}, J=6.6$ $\mathrm{Hz}, 2 \mathrm{H}), 2.14$ (s, 1H); ${ }^{13} \mathrm{C}$ NMR (125 MHz, DMSO-d 6 ) $\delta$ 206.3, 153.3, 146.5, 127.0, 123.4, 68.1, 52.4, 30.4; HRMS calcd for $\mathrm{C}_{10} \mathrm{H}_{11} \mathrm{NO}_{4}[\mathrm{M}+\mathrm{Na}]^{+} 232.0581$, found 232.0584.

\section{4-(1-Hydroxy-3-oxobutyl)benzonitrile (15b)}

Beige solid, $104 \mathrm{mg}$ (55\% yield); mp 83-88 ${ }^{\circ} \mathrm{C}$; Data for 15b: ${ }^{1} \mathrm{H}$ NMR (500 MHz, DMSO-d 6 ) $\delta 7.82$ $(\mathrm{d}, J=8.1 \mathrm{~Hz}, 2 \mathrm{H}), 7.59(\mathrm{~d}, J=8.0 \mathrm{~Hz}, 2 \mathrm{H}), 5.65(\mathrm{~d}, J=4.7 \mathrm{~Hz}, 1 \mathrm{H}), 5.10-5.13(\mathrm{~m}, 1 \mathrm{H}), 2.76(\mathrm{~d}, J=$ $5.8 \mathrm{~Hz}, 2 \mathrm{H}), 2.16$ (s, 3H); ${ }^{13} \mathrm{C}$ NMR (125 MHz, DMSO-d6) $\delta 151.1,132.2,126.8,119.0,109.7,68.3$, 52.4, 30.4; HRMS calcd for $\mathrm{C}_{11} \mathrm{H}_{11} \mathrm{NO}_{2}[\mathrm{M}+\mathrm{Na}]^{+} 212.0682$, found 212.0677 .

\section{General procedure for the preparation of $\beta$-hydroxy ketone derivatives 18a-b by Mukaiyama aldol reaction:}

To a solution of ketone $\mathbf{1 7}(1 \mathrm{mmol})$ in $10 \mathrm{~mL}$ of THF under argon at $-78{ }^{\circ} \mathrm{C}$ was slowly added a solution of $1 \mathrm{M}$ LiHMDS $(1.2 \mathrm{~mL}, 1.2 \mathrm{mmol})$ and the reaction mixture was stirred for 1 hour at $-78^{\circ} \mathrm{C}$, prior to the addition of chlorotrimethylsilane $(153 \mu \mathrm{L}, 1.2 \mathrm{mmol})$ in THF. The mixture was further stirred for 1 hour, then allowed to warm up to room temperature. The reaction was quenched with 10 $\mathrm{mL}$ of saturated aqueous $\mathrm{NH}_{4} \mathrm{Cl}$, after addition of EtOAc, the layers were separated, and the aqueous layer was extracted several times with EtOAc, dried over $\mathrm{Na}_{2} \mathrm{SO}_{4}$, and concentrated in vacuo to afford the corresponding silyl enol ether used directly without further purification for the next step.

To a solution of silyl enol ether in $10 \mathrm{~mL}$ of $\mathrm{CH}_{2} \mathrm{Cl}_{2}$ under argon at $-78{ }^{\circ} \mathrm{C}$ was added acetaldehyde (85 $\mu \mathrm{L}, 1.5 \mathrm{mmol})$ for $\mathbf{1 8 a}$ or propionaldehyde for $\mathbf{1 8 b}(110 \mu \mathrm{L}, 1.5 \mathrm{mmol})$ followed by a slow addition of $\mathrm{TiCl}_{4}(121 \mu \mathrm{L}, 1.1 \mathrm{mmol})$. The resulting red solution was stirred at $-78{ }^{\circ} \mathrm{C}$ for 5 hours and then the 
reaction was quenched with $10 \mathrm{~mL}$ of saturated aqueous $\mathrm{NH}_{4} \mathrm{Cl}$ and warmed to room temperature. The organic layer was separated and then washed with $15 \mathrm{~mL}$ of saturated aqueous $\mathrm{NaHCO}_{3}$. The aqueous layers were extracted with $20 \mathrm{~mL}$ of $\mathrm{CH}_{2} \mathrm{Cl}_{2}$. The combined organic layers were dried over $\mathrm{Na}_{2} \mathrm{SO}_{4}$ and concentrated in vacuo. The product was purified by flash column chromatography on silica gel $($ EtOAc/n-Hexane $=1: 3)$ to obtain the desired $\beta$-hydroxy ketone 18.

\section{3-Hydroxy-1-(thiophen-2-yl)butan-1-one (18a)}

Colorless oil, $107 \mathrm{mg}$ (63\% yield); Data for 18a: ${ }^{1} \mathrm{H}$ NMR $\left(500 \mathrm{MHz}, \mathrm{DMSO}-\mathrm{d}_{6}\right) \delta 7.98$ (d, $J=4.9 \mathrm{~Hz}$, 1H), $7.94(\mathrm{~d}, J=3.8 \mathrm{~Hz}, 1 \mathrm{H}), 7.24(\mathrm{t}, J=4.8 \mathrm{~Hz}, 1 \mathrm{H}), 4.70(\mathrm{~d}, J=4.9 \mathrm{~Hz}, 1 \mathrm{H}), 4.15-4.17(\mathrm{~m}, 1 \mathrm{H})$, 2.85-3.09 (m, 2H), $1.14(\mathrm{~d}, J=6.2 \mathrm{~Hz}, 3 \mathrm{H}) ;{ }^{13} \mathrm{C}$ NMR (125 MHz, DMSO-d 6$) \delta 192.1,144.7,134.9$, 133.6, 128.8, 63.8, 48.4, 23.8; HRMS calcd for $\mathrm{C}_{8} \mathrm{H}_{10} \mathrm{O}_{2} \mathrm{~S}[\mathrm{M}+\mathrm{Na}]^{+}$193.0294, found 193.0297.

\section{1-(4-Chlorophenyl)-3-hydroxypentan-1-one (18b)}

Yellow oil, $145 \mathrm{mg}$ (68\% yield); Data for 18b: ${ }^{1} \mathrm{H}$ NMR (500 MHz, DMSO-d6) $\delta 7.97$ (d, $J=8.5 \mathrm{~Hz}$, 2H), $7.58(\mathrm{~d}, J=8.5 \mathrm{~Hz}, 2 \mathrm{H}), 4.62$ (d, $J=5.5 \mathrm{~Hz}, 1 \mathrm{H}), 3.89-3.95(\mathrm{~m}, 1 \mathrm{H}), 3.90-3.94(\mathrm{~m}, 1 \mathrm{H}), 2.94-3.10$ (m, 2H), 1.40-1.48 (m, 2H), 0.89 (t, $J=7.4$ Hz, 3H); ${ }^{13} \mathrm{C}$ NMR (125 MHz, DMSO-d 6 ) $\delta$ 198.5, 138.0, 136.0, 130.1, 128.8, 68.6, 45.9, 30.2, 10.0; HRMS calcd for $\mathrm{C}_{11} \mathrm{H}_{13} \mathrm{ClO}_{2}[\mathrm{M}+\mathrm{Na}]^{+}$235.0497, found 235.0502 .

\section{General procedure for the preparation of $\alpha$-diazo-1,3-diketone 16a, 16b, 19a and 19b:}

To a solution of $\beta$-hydroxy ketone (15a, 15b, 18a, 18b) $(1 \mathrm{mmol})$ in $10 \mathrm{~mL}$ of acetonitrile under argon at $0{ }^{\circ} \mathrm{C}$ was added IBX (1-hydroxy-1,2-benziodoxol-3(1H)-one 1-oxide) (364 mg, $1.3 \mathrm{mmol}$ ). The mixture was refluxed for 2 hours and was allowed to warm to room temperature and then filtered. The filtrate was concentrated to afford the 1,3-diketone used directly without further purification for the next step.

To a solution of 1,3-diketone in $15 \mathrm{~mL}$ of acetonitrile under argon at $0{ }^{\circ} \mathrm{C}$ was added successively triethylamine $(181 \mu \mathrm{L}, 1.3 \mathrm{mmol})$ and $p$-acetamido benzene sulfonyl azide ( $p$-ABSA) (240 mg, 1 mmol). The mixture was stirred for 2 hours and was allowed to warm to room temperature and was diluted with a mixture of diethyl ether/hexane $(1 / 1,30 \mathrm{~mL})$ and then filtered. The filtrate was concentrated and the residue purified by flash column chromatography on silica gel (EtOAc/n-Hexane $=1: 4)$ to afford the $\alpha$-diazo-1,3-diketone $\mathbf{1 6 a}, \mathbf{1 6} \mathbf{b}, \mathbf{1 9 a}$ and $\mathbf{1 9} \mathbf{b}$. 


\section{2-Diazo-1-(4-nitrophenyl)butane-1,3-dione (16a)}

Yellow solid, $198 \mathrm{mg}$ (85\% yield); mp 84-87 ${ }^{\circ} \mathrm{C}$; Data for 16a: ${ }^{1} \mathrm{H}$ NMR (500 MHz, DMSO-d 6$) \delta 8.34$ $(\mathrm{d}, J=8.7 \mathrm{~Hz}, 2 \mathrm{H}), 7.95$ (d, $J=8.7 \mathrm{~Hz}, 2 \mathrm{H}), 2.46(\mathrm{~s}, 3 \mathrm{H}) ;{ }^{13} \mathrm{C}$ NMR $\left(125 \mathrm{MHz}, \mathrm{DMSO}-\mathrm{d}_{6}\right) \delta 188.8$, $184.0,149.3,142.9,129.1,123.9,84.8,28.7$.

\section{4-(2-Diazo-3-oxobutanoyl)benzonitrile (16b)}

Beige solid, $164 \mathrm{mg}$ (77\% yield); mp 106-110 ${ }^{\circ} \mathrm{C}$; Data for 16b: ${ }^{1} \mathrm{H}$ NMR (500 MHz, DMSO-d6) $\delta 8.01$ (d, $J=8.2 \mathrm{~Hz}, 2 \mathrm{H}), 7.87$ (d, $J=8.3 \mathrm{~Hz}, 2 \mathrm{H}), 2.45$ (s, 3H); ${ }^{13} \mathrm{C}$ NMR (125 MHz, DMSO-d 6$) \delta$ 189.0, $184.2,141.4,132.8,128.4,118.2,114.4,84.6,28.7$.

\section{2-Diazo-1-(thiophen-2-yl)butane-1,3-dione (19a)}

Yellow oil, $169 \mathrm{mg}$ (87\% yield); Data for 19a: ${ }^{1} \mathrm{H} \mathrm{NMR}\left(600 \mathrm{MHz}, \mathrm{CDCl}_{3}\right) \delta$ 7.69-7.70 (m, 1H), 7.60$7.61(\mathrm{~m}, 1 \mathrm{H}), 7.15-7.16(\mathrm{~m}, 1 \mathrm{H}), 2.60(\mathrm{~s}, 3 \mathrm{H}) ;{ }^{13} \mathrm{C} \mathrm{NMR}\left(150 \mathrm{MHz}, \mathrm{CDCl}_{3}\right) \delta 190.8,175.1,141.9$, $133.5,130.4,127.9,81.7,29.3$.

\section{1-(4-Chlorophenyl)-2-diazopentane-1,3-dione (19b)}

Pale yellow solid, $192 \mathrm{mg}$ (81\% yield); mp 88-92 ${ }^{\circ} \mathrm{C}$; Data for 19b: ${ }^{1} \mathrm{H}$ NMR (500 MHz, DMSO-d6) $\delta 7.75(\mathrm{~d}, J=8.5 \mathrm{~Hz}, 2 \mathrm{H}), 7.59$ (d, $J=8.5 \mathrm{~Hz}, 2 \mathrm{H}), 2.86$ (q, $J=7.3 \mathrm{~Hz}, 2 \mathrm{H}), 1.05$ (t, $J=7.3 \mathrm{~Hz}, 3 \mathrm{H})$;

${ }^{13} \mathrm{C}$ NMR (125 MHz, DMSO-d6) $\delta$ 192.6, 184.1, 137.2, 136.4, 129.6, 128.8, 83.2, 33.8, 8.2.

\section{2-Diazo-1-phenylbutane-1,3-dione (21)}

To a solution of compound $20(3 \mathrm{~g}, 18.50 \mathrm{mmol})$ in $50 \mathrm{~mL}$ of acetonitrile under argon at $0{ }^{\circ} \mathrm{C}$ was added successively triethylamine $(3.3 \mathrm{~mL}, 24.05 \mathrm{mmol})$ and $p$-acetamido benzene sulfonyl azide ( $p$ ABSA) (4.4 g, $18.50 \mathrm{mmol}$ ). The mixture was stirred for 2 hours and was allowed to warm to room temperature and was diluted with $100 \mathrm{~mL}$ of $\mathrm{Et}_{2} \mathrm{O} / \mathrm{n}$-Hexane $(1: 1)$ and then filtered. The filtrate was concentrated and the residue purified by flash column chromatography on silica gel (EtOAc/n-Hexane $=1: 3)$ to afford the desired compound 21 ( $3.1 \mathrm{~g}, 16.45 \mathrm{mmol}, 89 \%$ yield) as yellow solid; $\mathrm{mp} 78-81{ }^{\circ} \mathrm{C}$; Data for 21: ${ }^{1} \mathrm{H}$ NMR (500 MHz, DMSO-d6) $\delta 7.73$ (d, $\left.J=7.2 \mathrm{~Hz}, 2 \mathrm{H}\right), 7.62$ (t, $\left.J=7.4 \mathrm{~Hz}, 1 \mathrm{H}\right), 7.52-$ 7.55 (m, 2H), 2.46 (s, 3H); ${ }^{13} \mathrm{C}$ NMR (125 MHz, DMSO-d6) $\delta 189.8,185.0,137.5,132.5,128.8,127.6$, 83.6, 28.8. 


\section{General procedure for the preparation of $22 \mathrm{~b}-\mathrm{j}$ by titanium aldol reaction:}

To a solution of $\alpha$-diazo-1,3-diketone (16a, 16b, 19a, 19b and 21) (1 mmol) in $10 \mathrm{~mL}$ of $\mathrm{CH}_{2} \mathrm{Cl}_{2}$ under argon at $-78{ }^{\circ} \mathrm{C}$ was added dropwise $\mathrm{TiCl}_{4}(121 \mu \mathrm{L}, 1.1 \mathrm{mmol})$ followed by $\mathrm{Et}_{3} \mathrm{~N}(153 \mu \mathrm{L}, 1.1 \mathrm{mmol})$. The resulting red solution was stirred at $-78^{\circ} \mathrm{C}$ for 1 hour, after which a solution of aldehyde $(0.9$ mmol) in $\mathrm{CH}_{2} \mathrm{Cl}_{2}$ was slowly added. The reaction mixture was stirred at $-78{ }^{\circ} \mathrm{C}$ for 4 hours and then the reaction was quenched with $10 \mathrm{~mL}$ of saturated aqueous $\mathrm{NH}_{4} \mathrm{Cl}$ and warmed to room temperature. The organic layer was separated and then washed with $15 \mathrm{~mL}$ of saturated aqueous $\mathrm{NaHCO}_{3}$. The aqueous layers were extracted with $20 \mathrm{~mL}$ of $\mathrm{CH}_{2} \mathrm{Cl}_{2}$. The combined organic layers were dried over $\mathrm{Na}_{2} \mathrm{SO}_{4}$ and concentrated in vacuo. The product was purified by flash column chromatography on silica gel $($ EtOAc/n-Hexane $=1: 3)$ to afford the aldol product 22.

\section{2-Diazo-5-hydroxy-1-(4-nitrophenyl)heptane-1,3-dione (22b)}

Yellow oil, $201 \mathrm{mg}$ (69\% yield); Data for 22b: ${ }^{1} \mathrm{H}$ NMR (300 MHz, DMSO-d6) $\delta 8.33$ (d, J=8.1 Hz, 2H), $7.92(\mathrm{~d}, J=8.4 \mathrm{~Hz}, 2 \mathrm{H}), 4.75-4.77(\mathrm{~m}, 1 \mathrm{H}), 3.82-3.87(\mathrm{~m}, 1 \mathrm{H}), 2.86-2.91(\mathrm{~m}, 2 \mathrm{H}), 1.37-1.47$ (m, 2H), $0.88(\mathrm{t}, J=7.5 \mathrm{~Hz}, 3 \mathrm{H}) ;{ }^{13} \mathrm{C}$ NMR (75 MHz, DMSO-d 6$) \delta 190.0,184.3,149.3,143.1,129.1$, 123.8, 85.2, 68.4, 47.9, 30.0, 10.0; HRMS calcd for $\mathrm{C}_{13} \mathrm{H}_{13} \mathrm{~N}_{3} \mathrm{O}_{5}[\mathrm{M}+\mathrm{Na}]^{+} 314.0748$, found 314.0752.

\section{4-(2-Diazo-5-hydroxy-3-oxoheptanoyl)benzonitrile (22c)}

Yellow oil, $220 \mathrm{mg}$ (81\% yield); Data for 22c: ${ }^{1} \mathrm{H}$ NMR (600 MHz, DMSO-d6) $\delta 7.99$ (d, J=8.1 Hz, 2H), $7.84(\mathrm{~d}, J=8.5 \mathrm{~Hz}, 2 \mathrm{H}), 4.73-4.75(\mathrm{~m}, 1 \mathrm{H}), 3.85-3.87(\mathrm{~m}, 1 \mathrm{H}), 2.83-2.93(\mathrm{~m}, 2 \mathrm{H}), 1.30-1.46(\mathrm{~m}$, 2H), 0.87 (t, $J=7.4 \mathrm{~Hz}, 3 \mathrm{H}) ;{ }^{13} \mathrm{C}$ NMR (150 MHz, DMSO-d6) $\delta 190.1,184.5,141.5,132.7,128.4$, 118.2, 114.4, 84.8, 68.4, 47.9, 30.0, 10.0; HRMS calcd for $\mathrm{C}_{14} \mathrm{H}_{13} \mathrm{~N}_{3} \mathrm{O}_{3}[\mathrm{M}+\mathrm{Na}]^{+} 294.0849$, found 294.0849 .

\section{4-[2-Diazo-5-(2-fluorophenyl)-5-hydroxy-3-oxopentanoyl]benzonitrile (22d)}

Yellow oil, $169 \mathrm{mg}$ (50\% yield); Data for 22d: ${ }^{1} \mathrm{H}$ NMR $(500 \mathrm{MHz}, \mathrm{DMSO}-\mathrm{d} 6) \delta 7.99$ (d, J=8.6 Hz, 2H), $7.83(\mathrm{~d}, J=8.6 \mathrm{~Hz}, 2 \mathrm{H}), 7.52-7.56(\mathrm{~m}, 1 \mathrm{H}), 7.29-7.34(\mathrm{~m}, 1 \mathrm{H}), 7.20-7.23(\mathrm{~m}, 1 \mathrm{H})$, 7.12-7.16 (m, $1 \mathrm{H}), 5.63(\mathrm{~d}, J=4.9 \mathrm{~Hz}, 1 \mathrm{H}), 5.34-5.38(\mathrm{~m}, 1 \mathrm{H}), 3.36(\mathrm{~m}, 1 \mathrm{H}), 3.01(\mathrm{~m}, 1 \mathrm{H}) ;{ }^{13} \mathrm{C} \mathrm{NMR}(125 \mathrm{MHz}$, DMSO-d6) $\delta 189.0,184.4,160.0,158.0,141.3,132.8,131.7,129.0,128.4,127.9,124.5,118.2,115.1$, 114.4, 84.8, 62.8, 48.5; HRMS calcd for $\mathrm{C}_{18} \mathrm{H}_{12} \mathrm{FN}_{3} \mathrm{O}_{3}[\mathrm{M}+\mathrm{Na}]^{+}$360.0755, found 360.0761. 


\section{2-Diazo-5-hydroxy-1-(thiophen-2-yl)heptane-1,3-dione (22e)}

Yellow oil, $194 \mathrm{mg}$ (77\% yield); Data for 22e: ${ }^{1} \mathrm{H}$ NMR (500 MHz, DMSO-d6) $\delta$ 8.04-8.05 (m, 1H), 7.92-7.93 (m, 1H), 7.23-7.25 (m, 1H), 4.66 (d, $J=5.7 \mathrm{~Hz}, 1 \mathrm{H}), 3.84-3.88(\mathrm{~m}, 1 \mathrm{H}), 2.91-2.98(\mathrm{~m}, 2 \mathrm{H})$, 1.33-1.48 (m, 2H), $0.87(\mathrm{t}, J=7.4 \mathrm{~Hz}, 3 \mathrm{H}) ;{ }^{13} \mathrm{C}$ NMR (125 MHz, DMSO-d 6$) \delta 175.4,141.8,134.6$, 132.0, 128.4, 82.4, 68.3, 48.0, 30.0, 10.0; HRMS calcd for $\mathrm{C}_{11} \mathrm{H}_{12} \mathrm{~N}_{2} \mathrm{O}_{3} \mathrm{~S}[\mathrm{M}+\mathrm{Na}]^{+}$275.0461, found 275.0468 .

\section{1-(4-Chlorophenyl)-2-diazo-5-hydroxy-4-methylheptane-1,3-dione (22f)}

Yellow oil, $186 \mathrm{mg}$ (63\% yield); Inseparable diastereomeric mixture; Data for $22 \mathrm{f}$ (major isomer): ${ }^{1} \mathrm{H}$ NMR (300 MHz, $\left.\mathrm{CDCl}_{3}\right) \delta 8.01(\mathrm{~d}, J=8.7 \mathrm{~Hz}, 2 \mathrm{H}), 7.47(\mathrm{~d}, J=8.7 \mathrm{~Hz}, 2 \mathrm{H}), 4.60-4.67(\mathrm{~m}, 1 \mathrm{H}), 4.18$ $(\mathrm{d}, J=4.8 \mathrm{~Hz}, 1 \mathrm{H}), 3.10-3.16(\mathrm{~m}, 1 \mathrm{H}), 1.63-1.72(\mathrm{~m}, 2 \mathrm{H}), 0.98-1.20(\mathrm{~m}, 6 \mathrm{H}) ;{ }^{13} \mathrm{C} \mathrm{NMR}(75 \mathrm{MHz}$, $\left.\mathrm{CDCl}_{3}\right) \delta 191.3,171.6,140.3,133.5,130.5,128.8,84.3,57.4,36.3,23.0,13.2,10.0$; HRMS calcd for $\mathrm{C}_{14} \mathrm{H}_{15} \mathrm{ClN}_{2} \mathrm{O}_{3}[\mathrm{M}+\mathrm{Na}]^{+}$317.0664, found 317.0657 .

\section{2-Diazo-5-hydroxy-1-phenyl-5-(thiophen-2-yl)pentane-1,3-dione (22g)}

Yellow oil, $171 \mathrm{mg}$ (57\% yield); Data for 22g: ${ }^{1} \mathrm{H}$ NMR (300 MHz, DMSO-d6) $\delta 7.73$ (m, 2H), 7.60 (m, 1H), $7.51(\mathrm{t}, J=7.7 \mathrm{~Hz}, 2 \mathrm{H}), 7.41(\mathrm{dd}, J=4.9,1.3 \mathrm{~Hz}, 1 \mathrm{H}), 6.97-7.02(\mathrm{~m}, 2 \mathrm{H}), 5.88-5.90(\mathrm{~m}, 1 \mathrm{H})$, 5.38-5.40 (m, 1H), 3.24-3.46 (m, 2H); ${ }^{13} \mathrm{C}$ NMR (75 MHz, DMSO-d6) $\delta 189.7,185.1,149.7,137.5$, 132.6, 128.8, 127.7, 126.8, 124.5, 123.2, 83.9, 65.2, 50.4; HRMS calcd for $\mathrm{C}_{15} \mathrm{H}_{12} \mathrm{~N}_{2} \mathrm{O}_{3} \mathrm{~S}[\mathrm{M}+\mathrm{Na}]^{+}$ 323.0461, found 323.0460 .

\section{2-Diazo-5-hydroxy-1,5-diphenylpentane-1,3-dione (22h)}

Yellow oil, $153 \mathrm{mg}$ (52\% yield); Data for 22h: ${ }^{1} \mathrm{H}$ NMR (300 MHz, DMSO-d $) \delta$ 7.46-7.60 (m, 3H), 7.4-7.44 (m, 4H), 7.28-7.42 (m, 3H), $5.28(\mathrm{~m}, 1 \mathrm{H}), 3.56(\mathrm{~m}, 1 \mathrm{H}), 3.33-3.44(\mathrm{~m}, 2 \mathrm{H}) ;{ }^{13} \mathrm{C}$ NMR $(125$ MHz, DMSO-d6) $\delta 192.4,184.7,142.6,136.7,132.6,128.7,128.2,127.4,127.1,125.5,83.6,70.3$, 49.4; HRMS calcd for $\mathrm{C}_{17} \mathrm{H}_{14} \mathrm{~N}_{2} \mathrm{O}_{3}[\mathrm{M}+\mathrm{Na}]^{+} 317.0897$, found 317.0901 .

\section{5-Cyclohexyl-2-diazo-5-hydroxy-1-phenylpentane-1,3-dione (22i)}

Yellow oil, $237 \mathrm{mg}$ (79\% yield); Data for 22i: ${ }^{1} \mathrm{H}$ NMR (500 MHz, DMSO-d6) $\delta 7.71$ (d, J=7.3 Hz, 2H), $7.62(\mathrm{t}, J=7.4 \mathrm{~Hz}, 1 \mathrm{H}), 7.52(\mathrm{t}, J=7.6 \mathrm{~Hz}, 2 \mathrm{H}), 4.61$ (d, $J=5.8 \mathrm{~Hz}, 1 \mathrm{H}), 3.74-3.78(\mathrm{~m}, 1 \mathrm{H}), 2.90$ $(\mathrm{d}, J=5.7 \mathrm{~Hz}, 2 \mathrm{H}), 1.59-1.76(\mathrm{~m}, 5 \mathrm{H}), 0.95-1.28(\mathrm{~m}, 6 \mathrm{H}) ;{ }^{13} \mathrm{C}$ NMR $\left(125 \mathrm{MHz}, \mathrm{DMSO}-\mathrm{d}_{6}\right) \delta 191.4$, 
185.2, 137.6, 132.5, 128.7, 127.7, 83.6, 71.0, 45.5, 43.7, 28.8, 27.6, 26.2, 25.9, 25.8; HRMS calcd for $\mathrm{C}_{17} \mathrm{H}_{20} \mathrm{~N}_{2} \mathrm{O}_{3}[\mathrm{M}+\mathrm{Na}]^{+}$323.1366, found 323.1363.

\section{2-Diazo-5-hydroxy-6-methyl-1-phenylheptane-1,3-dione (22j)}

Yellow oil, $221 \mathrm{mg}$ (85\% yield); Data for 22j: ${ }^{1} \mathrm{H}$ NMR (500 MHz, DMSO-d6) $\delta 7.71$ (d, J=7.3 Hz, 2H), 7.62 (t, $J=7.5 \mathrm{~Hz}, 1 \mathrm{H}), 7.52$ (t, $J=7.6 \mathrm{~Hz}, 2 \mathrm{H}), 4.65$ (br. s, 1H), 3.76-3.78 (m, 1H), 2.85-2.95 (m, 2H), 1.60-1.64 (m, 1H), $0.85(\mathrm{~m}, 6 \mathrm{H}) ;{ }^{13} \mathrm{C}$ NMR (125 MHz, DMSO-d 6$) \delta$ 191.3, 185.2, 137.6, 132.5, 128.7, 127.7, 83.7, 71.5, 45.4, 33.6, 18.7, 17.5; HRMS calcd for $\mathrm{C}_{14} \mathrm{H}_{16} \mathrm{~N}_{2} \mathrm{O}_{3}[\mathrm{M}+\mathrm{Na}]^{+} 283.1053$, found 283.1052.

\section{General procedure for the preparation of pyridazine derivatives $24 \mathrm{~b}-\mathrm{j}$ :}

To a solution of $22(1 \mathrm{mmol})$ in $10 \mathrm{~mL}$ of acetonitrile under argon was added IBX (1-hydroxy-1,2benziodoxol-3(1H)-one 1-oxide) (364 mg, $1.3 \mathrm{mmol})$. The mixture was refluxed for 2 hours and was allowed to warm to room temperature and then filtered. The filtrate was concentrated and used directly without further purification for the next step.

To the previously prepared crude in $15 \mathrm{~mL}$ of $i-\operatorname{Pr}_{2} \mathrm{O}$ was added $\mathrm{P}(n-\mathrm{Bu})_{3}(250 \mu \mathrm{L}, 1.0 \mathrm{mmol})$. The reaction mixture was stirred under argon at room temperature for 30 minutes, which after this time a precipitate was formed. The suspension was filtered, washed with $i-\operatorname{Pr}_{2} \mathrm{O}$ and dried to afford $\mathbf{2 4 b}, \mathbf{2 4 c}$, 24e, 24f and 24j. 24d, 24g, 24h and 24i were purified by flash column chromatography on silica gel $($ EtOAc/n-Hexane $=8: 2)$ due to no formation of precipitate.

\section{6-Ethyl-3-(4-nitrobenzoyl)pyridazin-4-ol (24b)}

Brown solid, $164 \mathrm{mg}$ (60\% yield); mp 241-244 ${ }^{\circ} \mathrm{C}$; Data for $24 \mathbf{b}:{ }^{1} \mathrm{H}$ NMR (500 MHz, DMSO-d6) $\delta$ 13.54 (br. s, 1H), 8.34 (d, $J=8.5 \mathrm{~Hz}, 2 \mathrm{H}), 8.05$ (d, $J=8.4 \mathrm{~Hz}, 2 \mathrm{H}), 6.47$ (s, 1H), 2.63 (q, $J=7.4 \mathrm{~Hz}$, 2H), $1.24(\mathrm{t}, J=7.6 \mathrm{~Hz}, 3 \mathrm{H}) ;{ }^{13} \mathrm{C}$ NMR (125 MHz, DMSO-d 6$) \delta 157.0,152.7,150.4,140.2,130.6$, 124.1, 116.5, 24.3, 12.5; HRMS calcd for $\mathrm{C}_{13} \mathrm{H}_{11} \mathrm{~N}_{3} \mathrm{O}_{4}[\mathrm{M}+\mathrm{H}]^{+}$274.0822, found 274.0829.

\section{4-(6-Ethyl-4-hydroxypyridazine-3-carbonyl)benzonitrile (24c)}

Yellow solid, $144 \mathrm{mg}$ (57\% yield); mp 233-235 ${ }^{\circ} \mathrm{C}$; Data for $24 \mathrm{c}:{ }^{1} \mathrm{H}$ NMR $(600 \mathrm{MHz}$, DMSO-d 6$) \delta$ 13.55 (br. s, 1H), 8.02 (d, $J=8.3 \mathrm{~Hz}, 2 \mathrm{H}), 7.96$ (d, $J=8.3 \mathrm{~Hz}, 2 \mathrm{H}), 6.46$ (s, 1H), 2.62 (q, $J=7.6 \mathrm{~Hz}$, 2H), $1.24(\mathrm{t}, J=7.6 \mathrm{~Hz}, 3 \mathrm{H}) ;{ }^{13} \mathrm{C}$ NMR (150 MHz, DMSO-d 6$) \delta 191.9,169.1,156.8,152.8,138.7$, 
133.0, 129.8, 118.1, 116.3, 115.9, 24.1, 12.5; HRMS calcd for $\mathrm{C}_{14} \mathrm{H}_{11} \mathrm{~N}_{3} \mathrm{O}_{2}[\mathrm{M}+\mathrm{H}]^{+} 254.0924$, found 254.0928.

\section{4-[6-(4-Fluorophenyl)-4-hydroxypyridazine-3-carbonyl]benzonitrile (24d)}

Beige solid, $144 \mathrm{mg}$ (45\% yield); mp 237-240 ${ }^{\circ} \mathrm{C}$; Data for $24 \mathrm{~d}:{ }^{1} \mathrm{H}$ NMR (600 MHz, DMSO-d6) $\delta$ 13.94 (br. s, 1H), 8.05 (m, 4H), 7.73-7.76 (m, 1H), 7.66-7.69 (m, 1H), 7.46-7.49 (m, 1H), 7.43-7.45 (m, 1H), $6.78(\mathrm{~s}, 1 \mathrm{H}) ;{ }^{13} \mathrm{C}$ NMR (150 MHz, DMSO-d 6$) \delta 191.7,159.9,158.3,152.9,138.7,133.4,133.3$, 133.0, 130.9, 130.0, 125.9, 118.9, 118.2, 116.6, 116.5, 116.0; HRMS calcd for $\mathrm{C}_{18} \mathrm{H}_{10} \mathrm{FN}_{3} \mathrm{O}_{2}[\mathrm{M}+\mathrm{H}]^{+}$ 320.0830 , found 320.0831 .

\section{6-Ethyl-3-(thiophene-2-carbonyl)pyridazin-4-ol (24e)}

Orange solid, $129 \mathrm{mg}$ (55\% yield); mp 236-239 ${ }^{\circ} \mathrm{C}$; Data for 24e: ${ }^{1} \mathrm{H}$ NMR (600 MHz, DMSO-d6) $\delta 13.41$ (br. s, $1 \mathrm{H}), 8.13-8.14(\mathrm{~m}, 1 \mathrm{H}), 7.69-7.70(\mathrm{~m}, 1 \mathrm{H}), 7.24-7.26(\mathrm{~m}, 1 \mathrm{H}), 6.42(\mathrm{~s}, 1 \mathrm{H}), 2.61(\mathrm{q}, J=$ $7.6 \mathrm{~Hz}, 2 \mathrm{H}), 1.23$ (t, $J=7.7 \mathrm{~Hz}, 3 \mathrm{H}) ;{ }^{13} \mathrm{C}$ NMR (150 MHz, DMSO-d 6$) \delta 184.0,169.1,156.5,153.0$, 142.2, 136.8, 136.5, 129.1, 116.0, 24.1, 12.5; HRMS calcd for $\mathrm{C}_{11} \mathrm{H}_{10} \mathrm{~N}_{2} \mathrm{O}_{2} \mathrm{~S}[\mathrm{M}+\mathrm{H}]^{+}$235.0536, found 235.0540 .

\section{3-(4-Chlorobenzoyl)-6-ethyl-5-methylpyridazin-4-ol (24f)}

Colorless oil, $158 \mathrm{mg}$ (57\% yield); Data for 24f: ${ }^{1} \mathrm{H}$ NMR (600 MHz, DMSO-d6) $\delta 13.37$ (br. s, 1H), $7.81(\mathrm{~d}, J=8.4 \mathrm{~Hz}, 2 \mathrm{H}), 7.61(\mathrm{~d}, J=8.5 \mathrm{~Hz}, 2 \mathrm{H}), 2.69(\mathrm{q}, J=7.3 \mathrm{~Hz}, 2 \mathrm{H}), 1.95(\mathrm{~s}, 3 \mathrm{H}), 1.22(\mathrm{t}, J=7.5$ $\mathrm{Hz}, 3 \mathrm{H}) ;{ }^{13} \mathrm{C}$ NMR (150 MHz, DMSO-d6) $\delta 191.9,168.6,153.1,150.6,139.0,134.4,131.2,129.1$, 125.5, 22.6, 12.7, 9.1; HRMS calcd for $\mathrm{C}_{14} \mathrm{H}_{13} \mathrm{ClN}_{2} \mathrm{O}_{2}[\mathrm{M}+\mathrm{H}]^{+}$277.0738, found 277.0739.

\section{3-Benzoyl-6-(thiophen-2-yl)pyridazin-4-ol (24g)}

Orange solid, $150 \mathrm{mg}$ (53\% yield); mp 206-209 ${ }^{\circ} \mathrm{C}$; Data for 24g: ${ }^{1} \mathrm{H}$ NMR (600 MHz, DMSO-d6) $\delta$ 7.88-7.92 (m, 4H), $7.72(\mathrm{t}, J=7.4 \mathrm{~Hz}, 1 \mathrm{H}), 7.57(\mathrm{t}, J=7.7 \mathrm{~Hz}, 2 \mathrm{H}), 7.29-7.31(\mathrm{~m}, 1 \mathrm{H}), 6.94$ (br. s, $1 \mathrm{H}) ;{ }^{13} \mathrm{C}$ NMR (150 MHz, DMSO-d 6$) \delta 192.4,135.5,134.3,131.0,129.6$, 129.0, 128.9; HRMS calcd for $\mathrm{C}_{15} \mathrm{H}_{10} \mathrm{~N}_{2} \mathrm{O}_{2} \mathrm{~S}[\mathrm{M}+\mathrm{H}]^{+}$283.0536, found 283.0534.

\section{3-Benzoyl-6-phenylpyridazin-4-ol (24h)}

Brown solid, $133 \mathrm{mg}$ (48\% yield); mp 201-204 ${ }^{\circ} \mathrm{C}$; Data for 24h: ${ }^{1} \mathrm{H}$ NMR (600 MHz, DMSO-d6) $\delta$ $7.89(\mathrm{~d}, J=8.4 \mathrm{~Hz}, 2 \mathrm{H}), 7.84(\mathrm{~d}, J=7.8 \mathrm{~Hz}, 2 \mathrm{H}), 7.72(\mathrm{t}, J=7.4 \mathrm{~Hz}, 1 \mathrm{H}), 7.56-7.62(\mathrm{~m}, 5 \mathrm{H}), 6.85$ (s, 
$1 \mathrm{H}) ;{ }^{13} \mathrm{C}$ NMR (150 MHz, DMSO-d 6$) \delta$ 192.6, 135.4, 134.3, 131.2, 129.5, 129.3, 129.0, 127.4, 115.3; HRMS calcd for $\mathrm{C}_{17} \mathrm{H}_{12} \mathrm{~N}_{2} \mathrm{O}_{2}[\mathrm{M}+\mathrm{H}]^{+} 277.0971$, found 277.0978 .

\section{3-Benzoyl-6-cyclohexylpyridazin-4-ol (24i)}

Beige solid, $147 \mathrm{mg}$ (52\% yield); mp 233-235 ${ }^{\circ} \mathrm{C}$; Data for 24i: ${ }^{1} \mathrm{H}$ NMR (600 MHz, DMSO-d 6$) \delta$ 13.36 (br. s, 1H), 7.81 (d, $J=8.3 \mathrm{~Hz}, 2 \mathrm{H}), 7.69$ (t, $J=7.4 \mathrm{~Hz}, 1 \mathrm{H}), 7.55$ (t, $J=8.1 \mathrm{~Hz}, 2 \mathrm{H}), 6.40$ (s, $1 \mathrm{H}), 2.54-2.59(\mathrm{~m}, 1 \mathrm{H}), 1.69-1.90(\mathrm{~m}, 5 \mathrm{H}), 1.33-1.52(\mathrm{~m}, 5 \mathrm{H}) ;{ }^{13} \mathrm{C}$ NMR $\left(150 \mathrm{MHz}, \mathrm{DMSO}-\mathrm{d}_{6}\right)$ $\delta 192.7,159.5,154.1,135.4,134.3,129.4,129.0,114.2,40.1,31.0,25.7,25.1$; HRMS calcd for $\mathrm{C}_{17} \mathrm{H}_{18} \mathrm{~N}_{2} \mathrm{O}_{2}[\mathrm{M}+\mathrm{H}]^{+} 283.1441$, found 283.1440.

\section{3-Benzoyl-6-(propan-2-yl)pyridazin-4-ol (24j)}

Beige solid, $133 \mathrm{mg}$ (55\% yield); mp 228-231 ${ }^{\circ} \mathrm{C}$; Data for $24 \mathrm{j}:{ }^{1} \mathrm{H}$ NMR (600 MHz, DMSO-d6) $\delta$ 13.41 (br. s, 1H), 7.81 (d, $J=8.2 \mathrm{~Hz}, 2 \mathrm{H}), 7.70$ (t, $J=7.4 \mathrm{~Hz}, 1 \mathrm{H}), 7.55$ (t, $J=7.9 \mathrm{~Hz}, 2 \mathrm{H}), 6.43$ (s, 1H), 2.88-2.93 (m, 1H), $1.27(\mathrm{~d}, J=7.1 \mathrm{~Hz}, 6 \mathrm{H}) ;{ }^{13} \mathrm{C}$ NMR (150 MHz, DMSO-d6) $\delta 192.7,169.3$, $160.5,154.2,135.4,134.3,129.4,129.0,113.8,30.3,21.1$; HRMS calcd for $\mathrm{C}_{14} \mathrm{H}_{14} \mathrm{~N}_{2} \mathrm{O}_{2}[\mathrm{M}+\mathrm{H}]^{+}$ 243.1128 , found 243.1130 .

\section{Method A: preparation of pyrazolo[4,3-c]pyridazine analogues 25a-f:}

A solution of $24(1 \mathrm{mmol})$ in $5 \mathrm{~mL}$ of $\mathrm{POCl}_{3}$ was heated at $95{ }^{\circ} \mathrm{C}$ for 4 hours, after which the reaction mixture was cooled down to room temperature. $\mathrm{POCl}_{3}$ was evaporated and the residue was dissolved in EtOAc and washed 2 times with $20 \mathrm{~mL}$ of saturated aqueous $\mathrm{NaHCO}_{3}$. The organic layer was dried over $\mathrm{Na}_{2} \mathrm{SO}_{4}$ and concentrated in vacuo to afford the chlorinated pyridazine used directly without further purification for the next step.

To a solution of chlorinated pyridazine in $5 \mathrm{~mL}$ of EtOH was added hydrazine hydrate $64 \%(63 \mu \mathrm{L}, 1.3$ mmol) followed by $\mathrm{Et}_{3} \mathrm{~N}(181 \mu \mathrm{L}, 1.3 \mathrm{mmol})$ and refluxed for 4 hours. After completion of the reaction (monitored by TLC), the reaction mixture was allowed to cool to room temperature. $\mathrm{CH}_{2} \mathrm{Cl}_{2}$ was added and the organic layer was then washed 2 times with $10 \mathrm{~mL}$ of saturated aqueous $\mathrm{NaHCO}_{3}$, and 1 time with $10 \mathrm{~mL}$ of saturated aqueous $\mathrm{NaCl}$. The organic layer was dried over $\mathrm{Na}_{2} \mathrm{SO}_{4}$ and concentrated in vacuo, the residue was purified by flash column chromatography on silica gel (EtOAc) to obtain the desired pyrazolo[4,3-c]pyridazine 25. 


\section{4-\{6-Ethyl-1H-pyrazolo[4,3-c]pyridazin-3-yl\}benzonitrile (25a)}

Beige solid, $207 \mathrm{mg}$ (83\% yield); $\mathrm{mp} 336-338^{\circ} \mathrm{C}$; Data for 25a: ${ }^{1} \mathrm{H}$ NMR (600 MHz, DMSO-d $) \delta 8.76$ $(\mathrm{d}, J=8.5 \mathrm{~Hz}, 2 \mathrm{H}), 8.05(\mathrm{~d}, J=8.5 \mathrm{~Hz}, 2 \mathrm{H}), 7.80(\mathrm{~s}, 1 \mathrm{H}), 3.16(\mathrm{q}, J=7.6 \mathrm{~Hz}, 2 \mathrm{H}), 1.38(\mathrm{t}, J=7.4 \mathrm{~Hz}$, $3 \mathrm{H}) ;{ }^{13} \mathrm{C}$ NMR (150 MHz, DMSO-d6) $\delta$ 159.0, 144.1, 141.0, 135.9, 133.8, 133.0, 127.3, 118.9, 111.1 , 105.2, 28.9, 14.6; HRMS calcd for $\mathrm{C}_{14} \mathrm{H}_{11} \mathrm{~N}_{5}[\mathrm{M}+\mathrm{H}]^{+}$250.1087, found 250.1089.

\section{4-[6-(2-Fluorophenyl)-1H-pyrazolo[4,3-c]pyridazin-3-yl]benzonitrile (25b)}

White solid, $249 \mathrm{mg}$ (79\% yield); mp 390-393 ${ }^{\circ} \mathrm{C}$; Data for $25 \mathbf{b}:{ }^{1} \mathrm{H}$ NMR (300 MHz, DMSO-d6) $\delta$ 14.25 (br. s, $1 \mathrm{H}), 8.78$ (d, $J=8.3 \mathrm{~Hz}, 2 \mathrm{H}), 8.25$ (s, 1H), 8.05-8.14 (m, 3H), 7.59-7.61 (m, 1H), 7.45 (t, $J$ $=8.4 \mathrm{~Hz}, 2 \mathrm{H}) ;{ }^{13} \mathrm{C}$ NMR $(75 \mathrm{MHz}$, DMSO-d 6$) \delta 161.7,158.4,149.6,144.2,141.3,135.7,133.3,133.1$, 131.7, 127.4, 125.1, 118.9, 116.7, 116.4, 111.4, 107.8; HRMS calcd for $\mathrm{C}_{18} \mathrm{H}_{10} \mathrm{FN} 5[\mathrm{M}+\mathrm{H}]^{+} 316.0993$, found 316.0989 .

\section{3-(4-Chlorophenyl)-6-ethyl-7-methyl-1H-pyrazolo[4,3-c]pyridazine (25c)}

Beige solid, $237 \mathrm{mg}$ (87\% yield); mp 323-327 ${ }^{\circ} \mathrm{C}$; Data for 25c: ${ }^{1} \mathrm{H}$ NMR (500 MHz, DMSO-d 6 ) $\delta 8.64$ $(\mathrm{d}, J=8.6 \mathrm{~Hz}, 2 \mathrm{H}), 7.68(\mathrm{~d}, J=8.6 \mathrm{~Hz}, 2 \mathrm{H}), 3.17$ (q, $J=7.5 \mathrm{~Hz}, 2 \mathrm{H}), 2.61(\mathrm{~s}, 3 \mathrm{H}), 1.37$ (t, $J=7.6 \mathrm{~Hz}$, $3 \mathrm{H}) ;{ }^{13} \mathrm{C}$ NMR (125 MHz, DMSO-d 6$) \delta 157.2,143.6,141.6,134.7,133.5,130.6,129.1,128.4,115.9$, 25.6, 13.9, 12.0; HRMS calcd for $\mathrm{C}_{14} \mathrm{H}_{13} \mathrm{ClN}_{4}[\mathrm{M}+\mathrm{H}]^{+} 273.0901$, found 273.0905.

\section{3-Phenyl-6-(thiophen-2-yl)-1H-pyrazolo[4,3-c]pyridazine (25d)}

Pale yellow solid, $225 \mathrm{mg}$ (81\% yield); mp 345-348 ${ }^{\circ} \mathrm{C}$; Data for 25d: ${ }^{1} \mathrm{H}$ NMR (600 MHz, DMSO-d6) $\delta 13.94$ (br. s, 1H), 8.59 (d, $J=8.4 \mathrm{~Hz}, 2 \mathrm{H}), 8.35$ (s, 1H), 8.06 (d, $J=3.7 \mathrm{~Hz}, 1 \mathrm{H}), 7.76$ (d, $J=5.0 \mathrm{~Hz}$, 1H), $7.60(\mathrm{t}, J=7.9 \mathrm{~Hz}, 2 \mathrm{H}), 7.50(\mathrm{~m}, 1 \mathrm{H}), 7.26(\mathrm{~m}, 1 \mathrm{H}) ;{ }^{13} \mathrm{C}$ NMR (150 MHz, DMSO-d6) $\delta$ 148.9, $144.5,143.2$, 141.2, 133.5, 131.3, 129.4, 129.2, 129.0, 128.5, 127.0, 126.5, 101.3; HRMS calcd for $\mathrm{C}_{15} \mathrm{H}_{10} \mathrm{~N}_{4} \mathrm{~S}[\mathrm{M}+\mathrm{H}]^{+}$279.0699, found 279.0701.

\section{3,6-Diphenyl-1H-pyrazolo[4,3-c]pyridazine (25e)}

Beige solid, $215 \mathrm{mg}$ (79\% yield); mp 332-335 ${ }^{\circ} \mathrm{C}$; Data for 25e: ${ }^{1} \mathrm{H}$ NMR (600 MHz, DMSO-d6) $\delta$ 13.96 (br. s, 1H), 8.63 (d, J=7.3 Hz, 2H), 8.34 (s, 1H), 8.30 (d, $J=7.4 \mathrm{~Hz}, 2 \mathrm{H}), 7.59-7.62$ (m, 4H), 7.49-7.56 (m, 2H); ${ }^{13} \mathrm{C}$ NMR (150 MHz, DMSO-d 6$) \delta 152.7,144.6,143.0,137.0,133.8,131.4,129.6$, 129.2, 129.1, 129.0, 127.5, 127.0, 103.7; HRMS calcd for $\mathrm{C}_{17} \mathrm{H}_{12} \mathrm{~N}_{4}[\mathrm{M}+\mathrm{H}]^{+} 273.1135$, found 273.1133. 


\section{3-Phenyl-6-(propan-2-yl)-1H-pyrazolo[4,3-c]pyridazine (25f)}

Beige solid, $179 \mathrm{mg}$ (75\% yield); mp 272-275 ${ }^{\circ} \mathrm{C}$; Data for $25 f:{ }^{1} \mathrm{H}$ NMR (600 MHz, DMSO-d 6$) \delta$ 13.70 (br. s, 1H), 8.58 (d, $J=8.3 \mathrm{~Hz}, 2 \mathrm{H}), 7.71$ (s, 1H), 7.58 (t, $J=7.4 \mathrm{~Hz}, 2 \mathrm{H}), 7.48$ (t, J = 7.4 Hz, $1 \mathrm{H}), 3.46-3.51(\mathrm{~m}, 1 \mathrm{H}), 1.41(\mathrm{~d}, J=6.9 \mathrm{~Hz}, 6 \mathrm{H}) ;{ }^{13} \mathrm{C}$ NMR $\left(150 \mathrm{MHz}, \mathrm{DMSO}-\mathrm{d}_{6}\right) \delta 162.2,144.3$, $142.7,133.6,131.6,129.0,128.9,126.9,103.5,34.4,22.9$; HRMS calcd for $\mathrm{C}_{14} \mathrm{H}_{14} \mathrm{~N}_{4}[\mathrm{M}+\mathrm{H}]^{+}$ 239.1291 , found 239.1293 .

\section{Method B: preparation of thieno[3,2-c]pyridazine analogues 26a-d:}

A solution of $24(1 \mathrm{mmol})$ in $5 \mathrm{~mL}$ of $\mathrm{POCl}_{3}$ was heated at $95{ }^{\circ} \mathrm{C}$ for 4 hours, after which the reaction mixture was cooled down to room temperature. $\mathrm{POCl}_{3}$ was evaporated and the residue was dissolved in EtOAc and washed 2 times with $20 \mathrm{~mL}$ of saturated aqueous $\mathrm{NaHCO}_{3}$. The organic layer was dried over $\mathrm{Na}_{2} \mathrm{SO}_{4}$ and concentrated in vacuo to afford the chlorinated pyridazine used directly without further purification for the next step.

To a solution of chlorinated pyridazine in $5 \mathrm{~mL}$ of EtOH was added methyl thioglycolate $(134 \mu \mathrm{L}, 1.5$ mmol) followed by $\mathrm{Et}_{3} \mathrm{~N}(417 \mu \mathrm{L}, 3 \mathrm{mmol})$ and refluxed for 16 hours. After completion of the reaction (monitored by TLC), the reaction mixture was allowed to cool to room temperature. $\mathrm{CH}_{2} \mathrm{Cl}_{2}$ was added and the organic layer was then washed 2 times with $10 \mathrm{~mL}$ of saturated aqueous $\mathrm{NaHCO}_{3}$, and 1 time with $10 \mathrm{~mL}$ of saturated aqueous $\mathrm{NaCl}$. The organic layer was dried over $\mathrm{Na}_{2} \mathrm{SO}_{4}$ and concentrated in vacuo, the residue was purified by flash column chromatography on silica gel $($ EtOAc $/ n-H e x a n e=1: 9)$ to obtain the desired thieno[3,2-c]pyridazine 26.

\section{Ethyl 3-ethyl-7-(4-nitrophenyl)thieno[3,2-c]pyridazine-6-carboxylate (26a)}

Brown solid, $185 \mathrm{mg}$ (52\% yield); mp 143-145 ${ }^{\circ} \mathrm{C}$; Data for 26a: ${ }^{1} \mathrm{H}$ NMR (500 MHz, DMSO-d 6$) \delta$ $8.47(\mathrm{~s}, 1 \mathrm{H}), 8.36$ (d, $J=8.8 \mathrm{~Hz}, 2 \mathrm{H}), 7.89$ (d, $J=8.9 \mathrm{~Hz}, 2 \mathrm{H}), 4.25$ (q, $J=7.0 \mathrm{~Hz}, 2 \mathrm{H}), 3.12$ (q, $J=$ $7.6 \mathrm{~Hz}, 2 \mathrm{H}), 1.36(\mathrm{t}, J=7.6 \mathrm{~Hz}, 3 \mathrm{H}), 1.15(\mathrm{t}, J=7.1 \mathrm{~Hz}, 3 \mathrm{H}) ;{ }^{13} \mathrm{C}$ NMR (125 MHz, DMSO-d 6$)$ $\delta 161.1,159.6,156.4,147.5,139.4,139.0,138.0,134.4,132.3,122.7,119.6,62.3,28.8,14.2,13.8$; HRMS calcd for $\mathrm{C}_{17} \mathrm{H}_{15} \mathrm{~N}_{3} \mathrm{O}_{4} \mathrm{~S}[\mathrm{M}+\mathrm{H}]^{+}$358.0856, found 358.0860.

\section{Ethyl 3-ethyl-7-(thiophen-2-yl)thieno[3,2-c]pyridazine-6-carboxylate (26b)}

Beige solid, $121 \mathrm{mg}$ (38\% yield); mp 139-142 ${ }^{\circ} \mathrm{C}$; Data for 26b: ${ }^{1} \mathrm{H}$ NMR $\left(600 \mathrm{MHz}, \mathrm{CDCl}_{3}\right) \delta 7.81$ $7.83(\mathrm{~m}, 2 \mathrm{H}), 7.58-7.59(\mathrm{~m}, 1 \mathrm{H}), 7.19-7.21(\mathrm{~m}, 1 \mathrm{H}), 4.39$ (q, $J=7.1 \mathrm{~Hz}, 2 \mathrm{H}), 3.20$ (q, $J=7.7 \mathrm{~Hz}, 2 \mathrm{H})$, $1.47(\mathrm{t}, J=7.7 \mathrm{~Hz}, 3 \mathrm{H}), 1.35(\mathrm{t}, J=7.1 \mathrm{~Hz}, 3 \mathrm{H}) ;{ }^{13} \mathrm{C}$ NMR (150 MHz, DMSO-d 6$) \delta 161.8,159.6$, 
$156.3,137.9,134.5,131.7,131.5,131.0,128.7,126.5,118.1,62.3,29.4,14.0$; HRMS calcd for $\mathrm{C}_{15} \mathrm{H}_{14} \mathrm{~N}_{2} \mathrm{O}_{2} \mathrm{~S}_{2}[\mathrm{M}+\mathrm{H}]^{+} 319.0569$, found 319.0579 .

\section{Ethyl 7-phenyl-3-(thiophen-2-yl)thieno[3,2-c]pyridazine-6-carboxylate (26c)}

Yellow solid, $120 \mathrm{mg}$ (33\% yield); mp 157-161 ${ }^{\circ} \mathrm{C}$; Data for 26c: ${ }^{1} \mathrm{H}$ NMR (300 MHz, DMSO-d6) $\delta$ $9.03(\mathrm{~s}, 1 \mathrm{H}), 7.97(\mathrm{~d}, J=3.4 \mathrm{~Hz}, 1 \mathrm{H}), 7.81(\mathrm{~d}, J=4.8 \mathrm{~Hz}, 1 \mathrm{H}), 7.59-7.61(\mathrm{~m}, 2 \mathrm{H}), 7.51-7.53(\mathrm{~m}, 3 \mathrm{H})$, $7.29(\mathrm{~d}, J=4.6 \mathrm{~Hz}, 1 \mathrm{H}), 4.25(\mathrm{q}, J=7.0 \mathrm{~Hz}, 2 \mathrm{H}), 1.15(\mathrm{t}, J=7.1 \mathrm{~Hz}, 3 \mathrm{H}) ;{ }^{13} \mathrm{C}$ NMR $(75 \mathrm{MHz}, \mathrm{DMSO}-$ d6) $\delta 161.4,156.9,149.5,141.5,140.4,138.4,133.5,132.0,130.8,130.4,128.9,128.7,127.7,127.3$, 115.8, 62.1, 13.8; HRMS calcd for $\mathrm{C}_{19} \mathrm{H}_{14} \mathrm{~N}_{2} \mathrm{O}_{2} \mathrm{~S}_{2}[\mathrm{M}+\mathrm{H}]^{+} 367.0569$, found 367.0570 .

\section{Methyl 3,7-diphenylthieno[3,2-c]pyridazine-6-carboxylate (26d)}

Brown solid, $148 \mathrm{mg}$ (41\% yield); mp 140-144 ${ }^{\circ} \mathrm{C}$; Data for 26d: ${ }^{1} \mathrm{H}$ NMR (500 MHz, DMSO-d 6$) \delta$ $9.09(\mathrm{~s}, 1 \mathrm{H}), 8.25(\mathrm{~d}, J=7.3 \mathrm{~Hz}, 2 \mathrm{H}), 7.60-7.64(\mathrm{~m}, 4 \mathrm{H}), 7.53-7.57(\mathrm{~m}, 4 \mathrm{H}), 3.81(\mathrm{~s}, 3 \mathrm{H}) ;{ }^{13} \mathrm{C}$ NMR (125 MHz, DMSO-d6) $\delta 161.7,157.0,153.2,141.6,138.5,136.1,133.1,132.0,130.8,130.2,129.3$, 128.7, 127.7, 127.3, 117.9, 53.1; HRMS calcd for $\mathrm{C}_{20} \mathrm{H}_{14} \mathrm{~N}_{2} \mathrm{O}_{2} \mathrm{~S}[\mathrm{M}+\mathrm{H}]^{+} 347.0849$, found 347.0846.

\section{Method C: preparation of 5H,6H,7H-pyridazino[4,3-e][1,4]diazepine analogues 27a-h:}

A solution of $24(1 \mathrm{mmol})$ in $5 \mathrm{~mL}$ of $\mathrm{POCl}_{3}$ was heated at $95^{\circ} \mathrm{C}$ for 4 hours, after which the reaction mixture was cooled down to room temperature. $\mathrm{POCl}_{3}$ was evaporated and the residue was dissolved in EtOAc and washed 2 times with $20 \mathrm{~mL}$ of saturated aqueous $\mathrm{NaHCO}_{3}$. The organic layer was dried over $\mathrm{Na}_{2} \mathrm{SO}_{4}$ and concentrated in vacuo to afford the chlorinated pyridazine used directly without further purification for the next step.

To a solution of chlorinated pyridazine in $5 \mathrm{~mL}$ of EtOH was added ethylene diamine (87 $\mu \mathrm{L}, 1.3$ $\mathrm{mmol})$ followed by $\mathrm{Et}_{3} \mathrm{~N}(181 \mu \mathrm{L}, 1.3 \mathrm{mmol})$ and refluxed for 16 hours. After completion of the reaction (monitored by TLC), the reaction mixture was allowed to cool to room temperature. $\mathrm{CH}_{2} \mathrm{Cl}_{2}$ was added and the organic layer was then washed 2 times with $10 \mathrm{~mL}$ of saturated aqueous $\mathrm{NaHCO}_{3}$, and 1 time with $10 \mathrm{~mL}$ of saturated aqueous $\mathrm{NaCl}$. The organic layer was dried over $\mathrm{Na}_{2} \mathrm{SO}_{4}$ and concentrated in vacuo, the residue was purified by flash column chromatography on silica gel $(\mathrm{MeOH} / \mathrm{EtOAc}=1: 9)$ to obtain the desired 5H,6H,7H-pyridazino[4,3-e][1,4]diazepine 27. 


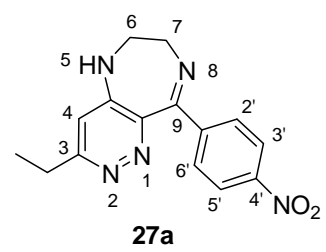

\section{3-Ethyl-9-(4-nitrophenyl)-5H,6H,7H-pyridazino[4,3-e][1,4]diazepine (27a)}

Pale yellow solid, $229 \mathrm{mg}$ (77\% yield); mp 213-215 ${ }^{\circ} \mathrm{C}$; Data for 27a: ${ }^{1} \mathrm{H}$ NMR (500 MHz, DMSO-d6) $\delta 8.21$ (d, $J=8.8$ Hz, 2H, H3', H5'), 7.65 (m, 3H, NH, H2', H6'), 6.72 (s, 1H, H4), 4.10-4.11 (m, 2H, H7), 3.51-3.52 (m, 2H, H6), 2.75 (q, $\left.J=7.6 \mathrm{~Hz}, 2 \mathrm{H}, \mathrm{CH}_{2}\right), 1.23\left(\mathrm{t}, J=7.6 \mathrm{~Hz}, 3 \mathrm{H}, \mathrm{CH}_{3}\right) ;{ }^{13} \mathrm{C} \mathrm{NMR}$ (125 MHz, DMSO-d6) $\delta 168.0$ (C9), 161.7 (C3), 148.6 (C4'), 147.3 (C), 146.6 (C), 139.8 (C1'), 130.3 (C2', C6'), 122.7 (C3', C5'), 109.9 (C4), 53.3 (C6), 47.5 (C7), $28.0\left(\mathrm{CH}_{2}\right), 13.4\left(\mathrm{CH}_{3}\right)$; HRMS calcd for $\mathrm{C}_{15} \mathrm{H}_{15} \mathrm{~N}_{5} \mathrm{O}_{2}[\mathrm{M}+\mathrm{H}]^{+} 298.1298$, found 298.1306.

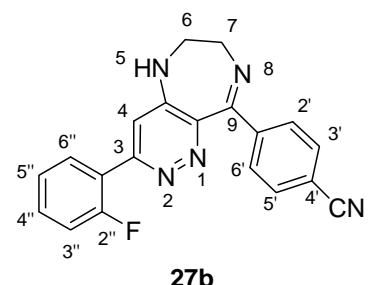

27b

\section{4-[3-(2-Fluorophenyl)-5H,6H,7H-pyridazino[4,3-e][1,4]diazepin-9-yl]benzonitrile (27b)}

White solid, $271 \mathrm{mg}$ (79\% yield); mp 251-255 ${ }^{\circ} \mathrm{C}$; Data for $27 \mathbf{b}:{ }^{1} \mathrm{H}$ NMR (500 MHz, DMSO-d 6$) \delta$ 8.02-8.05 (m, 1H, H6”), 7.96-7.97 (m, 1H, NH), 7.84 (d, J=8.2 Hz, 2H, H2', H6'), 7.64 (d, J = 7.8 Hz, 2H, H3', H5'), 7.53-7.57 (m, 1H, H4”), 7.36-7.41 (m, 2H, H3”, H5”), 7.32 (s, 1H, H4), 4.16-4.17 (m, 2H, H7), 3.56-3.57 (m, 2H, H6); ${ }^{13} \mathrm{C}$ NMR (125 MHz, DMSO-d6) $\delta 168.0$ (C9), 161.1 (C3), 159.1 (C2”), 151.8 (C), 146.5 (C), 139.8 (C1'), 131.7 (C4”), 131.5 (C3', C5'), 130.7 (C6”), 130.0 (C2', C6'), 125.0 (C5”), 124.3 (C1”), 118.9 (C3”), 116.5 (C $\equiv \mathrm{N}), 112.3$ (C4'), 111.0 (C4), 53.4 (C6), 47.6 (C7); HRMS calcd for $\mathrm{C}_{20} \mathrm{H}_{14} \mathrm{FN}_{5}[\mathrm{M}+\mathrm{H}]^{+}$344.1306, found 344.1301.

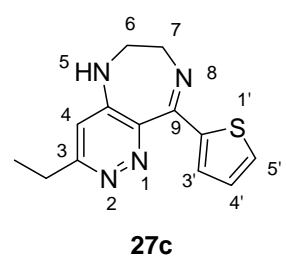

\section{3-Ethyl-9-(thiophen-2-yl)-5H,6H,7H-pyridazino[4,3-e][1,4]diazepine (27c)}

Yellow solid, $209 \mathrm{mg}$ (81\% yield); mp $185-188{ }^{\circ} \mathrm{C}$; Data for $27 \mathrm{c}:{ }^{1} \mathrm{H}$ NMR $(500 \mathrm{MHz}$, DMSO-d 6$) \delta$ 7.58 (d, $\left.J=4.9 \mathrm{~Hz}, 1 \mathrm{H}, \mathrm{H} 5^{\prime}\right), 7.36$ (d, $J=3.0 \mathrm{~Hz}, 1 \mathrm{H}, \mathrm{H} 3$ '), 7.31 (br. s, 1H, NH), 7.05 (t, $J=4.0 \mathrm{~Hz}$, 
1H, H4'), 6.69 (s, 1H, H4), 3.98-3.99 (m, 2H, H7), 3.52-3.53 (m, 2H, H6), 2.78 (q, J = 7.6 Hz, 2H, $\left.\mathrm{CH}_{2}\right), 1.26\left(\mathrm{t}, J=7.6 \mathrm{~Hz}, 3 \mathrm{H}, \mathrm{CH}_{3}\right) ;{ }^{13} \mathrm{C}$ NMR (125 MHz, DMSO-d6) $\delta 162.6$ (C9), 162.1 (C3), 146.0 (C), 145.1 (C), 139.9 (C2'), 130.5 (C3'), 129.2 (C4'), 127.2 (C5'), 110.2 (C4), 51.4 (C6), 48.3 (C7), $28.0\left(\mathrm{CH}_{2}\right), 13.4\left(\mathrm{CH}_{3}\right)$; HRMS calcd for $\mathrm{C}_{13} \mathrm{H}_{14} \mathrm{~N}_{4} \mathrm{~S}[\mathrm{M}+\mathrm{H}]^{+} 259.1012$, found 259.1007 .

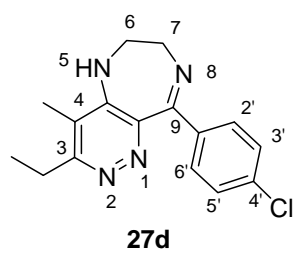

\section{9-(4-Chlorophenyl)-3-ethyl-4-methyl-5H,6H,7H-pyridazino[4,3-e][1,4]diazepine (27d)}

Beige solid, $247 \mathrm{mg}$ (82\% yield); mp 242-247 ${ }^{\circ} \mathrm{C}$; Data for $27 \mathrm{~d}:{ }^{1} \mathrm{H}$ NMR (300 MHz, DMSO-d 6$) \delta$ 7.38-7.39 (m, 4H, H2', H3', H5', H6'), 6.64 (br. s, 1H, NH), 4.01-4.04 (m, 2H, H7), 3.58-3.60 (m, 2H, H6), $2.86\left(\mathrm{q}, J=7.6 \mathrm{~Hz}, 2 \mathrm{H}, \mathrm{CH}_{2}\right), 2.09\left(\mathrm{~s}, 3 \mathrm{H}, \mathrm{CH}_{3}\right), 1.22\left(\mathrm{t}, J=7.5 \mathrm{~Hz}, 3 \mathrm{H}, \mathrm{CH}_{3}\right) ;{ }^{13} \mathrm{C}$ NMR $(75$ MHz, DMSO-d6) $\delta 168.6$ (C9), 159.7 (C3), 144.6 (C), 141.2 (C), 139.7 (C1'), 133.3 (C4'), 130.8 (C2', C6'), 127.5 (C3', C5'), 117.6 (C4), 52.5 (C6), 48.7 (C7), $26.7\left(\mathrm{CH}_{2}\right), 13.3\left(\mathrm{CH}_{3}\right), 11.7\left(\mathrm{CH}_{3}\right)$; HRMS calcd for $\mathrm{C}_{16} \mathrm{H}_{17} \mathrm{ClN}_{4}[\mathrm{M}+\mathrm{H}]^{+}$301.1214, found 301.1217.

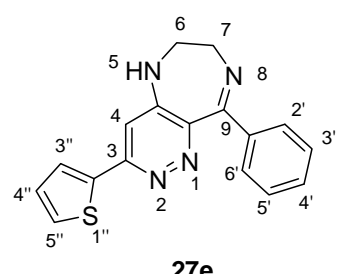

$27 e$

\section{9-Phenyl-3-(thiophen-2-yl)-5H,6H,7H-pyridazino[4,3-e][1,4]diazepine (27e)}

White solid, $242 \mathrm{mg}$ (79\% yield); mp 193-196 ${ }^{\circ} \mathrm{C}$; Data for 27e: ${ }^{1} \mathrm{H}$ NMR (600 MHz, DMSO-d6) $\delta$ 7.73-7.74 (m, 1H, H5”), 7.69-7.70 (m, 1H, H3”), 7.61-7.62 (m, 1H, NH), 7.34-7.44 (m, 5H, H2', H3', H4', H5', H6'), 7.25 (s, 1H, H4), 7.21-7.23 (m, 1H, H4”), 4.07-4.08 (m, 2H, H7), 3.56-3.58 (m, 2H, H6); ${ }^{13} \mathrm{C}$ NMR (150 MHz, DMSO-d6) $\delta 169.1$ (C9), 151.2 (C3), 146.7 (C), 141.8 (C), 141.0 (C2”), 140.5 (C1'), 129.5 (C4'), 129.1 (C2', C6'), 128.7 (C3”), 128.4 (C4”), 127.5 (C3', C5'), 126.1 (C5”), 106.2 (C4), 52.3 (C6), 48.3 (C7); HRMS calcd for $\mathrm{C}_{17} \mathrm{H}_{14} \mathrm{~N}_{4} \mathrm{~S}[\mathrm{M}+\mathrm{H}]^{+}$307.1012, found 307.1016. 


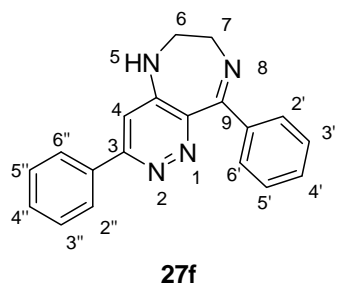

\section{3,9-Diphenyl-5H,6H,7H-pyridazino[4,3-e][1,4]diazepine (27f)}

Yellow solid, $231 \mathrm{mg}$ (77\% yield); mp 186-189 ${ }^{\circ} \mathrm{C}$; Data for $27 f:{ }^{1} \mathrm{H}$ NMR (600 MHz, DMSO-d6) $\delta$ 8.01 (m, 2H, H2', H6'), 7.60-7.61 (m, 1H, NH), 7.35-7.56 (m, 8H, H3', H4', H5', H2”, H3”, H4”, H5”, H6”), 7.31 (s, 1H, H4), 4.09-4.10 (m, 2H, H7), 3.58-3.60 (m, 2H, H6); ${ }^{13} \mathrm{C}$ NMR (150 MHz, DMSOd6) $\delta 169.3$ (C9), 155.3 (C3), 146.8 (C), 141.9 (C), 140.9 (C1'), 136.3 (C1”), 129.8 (C4'), 129.1 (C2', C6’), 129.0 (C3”, C5”), 128.7 (C4”), 127.5 (C3', C5'), 126.7 (C2”, C6”), 108.5 (C4), 52.4 (C6), 48.3 (C7); HRMS calcd for $\mathrm{C}_{19} \mathrm{H}_{16} \mathrm{~N}_{4}[\mathrm{M}+\mathrm{H}]^{+} 301.1448$, found 301.1446 .

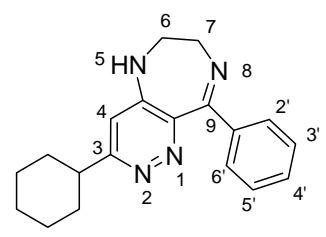

$27 \mathrm{~g}$

\section{3-Cyclohexyl-9-phenyl-5H,6H,7H-pyridazino[4,3-e][1,4]diazepine (27g)}

White solid, $242 \mathrm{mg}$ (79\% yield); mp $244-247{ }^{\circ} \mathrm{C}$; Data for $27 \mathrm{~g}:{ }^{1} \mathrm{H}$ NMR (500 MHz, DMSO-d6) $\delta$ 7.34-7.39 (m, 6H, NH, H2', H3', H4', H5', H6'), 6.67 (s, 1H, H4), 4.01-4.03 (m, 2H, H7), 3.51-3.52 (m, 2H, H6), 2.68-2.72 (m, 1H, CH), 1.70-1.90 (m, 5H, $\left.\mathrm{CH}_{2}, \mathrm{CH}_{2}, \mathrm{CH}\right), 1.24-1.50$ (m, 5H, $\mathrm{CH}_{2}, \mathrm{CH}_{2}$, $\mathrm{CH}) ;{ }^{13} \mathrm{C}$ NMR (125 MHz, DMSO-d6) $\delta 169.4$ (C9), 164.3 (C3), 146.7 (C), 142.0 (C), 140.6 (C1'), 129.1 (C2', C6’), 128.6 (C4'), 127.4 (C3', C5'), 108.9 (C4), 52.3 (C6), 48.3 (C7), 43.3 (CH), 32.2 $\left(2 \mathrm{xCH}_{2}\right), 26.1\left(2 \mathrm{xCH}_{2}\right), 25.8(\mathrm{CH})$; HRMS calcd for $\mathrm{C}_{19} \mathrm{H}_{22} \mathrm{~N}_{4}[\mathrm{M}+\mathrm{H}]^{+} 307.1917$, found 307.1909.

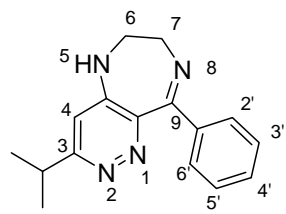

27h

\section{9-Phenyl-3-(propan-2-yl)-5H,6H,7H-pyridazino[4,3-e][1,4]diazepine (27h)}

White solid, $208 \mathrm{mg}$ (78\% yield); mp $208-212{ }^{\circ} \mathrm{C}$; Data for $27 \mathbf{h}:{ }^{1} \mathrm{H}$ NMR (500 MHz, DMSO-d6) $\delta$ 7.34-7.39 (m, 6H, NH, H2', H3', H4', H5', H6'), 6.70 (s, 1H, H4), 4.01-4.02 (m, 2H, H7), 3.52-3.53 (m, 2H, H6), 3.02-3.07 (m, 1H, CH), $1.25\left(\mathrm{~d}, J=6.9 \mathrm{~Hz}, 6 \mathrm{H}, 2 \mathrm{xCH}_{3}\right) ;{ }^{13} \mathrm{C}$ NMR (125 MHz, DMSO-d $)$ 
ઈ 169.4 (C9), 165.2 (C3), 146.7 (C), 142.0 (C), 140.6 (C1'), 129.1 (C2', C6'), 128.6 (C4'), 127.4 (C3', C5'), 108.5 (C4), $52.3(\mathrm{C} 6), 48.3(\mathrm{C} 7), 33.5(\mathrm{CH}), 22.2\left(2 \mathrm{xCH}_{3}\right)$; HRMS calcd for $\mathrm{C}_{16} \mathrm{H}_{18} \mathrm{~N}_{4}[\mathrm{M}+\mathrm{H}]^{+}$ 267.1604 , found 267.1602 .

\section{Method D: preparation of isoxazolo[4,5-c]pyridazine analogues 28a-b:}

A solution of $24(1 \mathrm{mmol})$ in $5 \mathrm{~mL}$ of $\mathrm{POCl}_{3}$ was heated at $95{ }^{\circ} \mathrm{C}$ for 4 hours, after which the reaction mixture was cooled down to room temperature. $\mathrm{POCl}_{3}$ was evaporated and the residue was dissolved in EtOAc and washed 2 times with $20 \mathrm{~mL}$ of saturated aqueous $\mathrm{NaHCO}_{3}$. The organic layer was dried over $\mathrm{Na}_{2} \mathrm{SO}_{4}$ and concentrated in vacuo to afford the chlorinated pyridazine used directly without further purification for the next step.

To a solution of chlorinated pyridazine in $5 \mathrm{~mL}$ of EtOH and $1 \mathrm{~mL}$ of pyridine was added hydroxylamine hydrochloride (208 $\mathrm{mg}, 3 \mathrm{mmol}$ ) and refluxed for 16 hours. After conversion of the ketone into the corresponding oxime (monitored by TLC), the reaction mixture was allowed to cool to room temperature. $\mathrm{CH}_{2} \mathrm{Cl}_{2}$ was added and the organic layer was then washed 2 times with $10 \mathrm{~mL}$ of saturated aqueous $\mathrm{NaHCO}_{3}$, and 1 time with $10 \mathrm{~mL}$ of saturated aqueous $\mathrm{NaCl}$. The organic layer was dried over $\mathrm{Na}_{2} \mathrm{SO}_{4}$ and concentrated in vacuo, to the residue obtained dissolved in THF was slowly added $\mathrm{NaH}$ 60\% (52 mg, $1.3 \mathrm{mmol}$ ) under argon at room temperature. The reaction mixture was heated at $40{ }^{\circ} \mathrm{C}$ for 2 hours, after completion of the reaction (monitored by TLC), the reaction mixture was allowed to cool to room temperature. $\mathrm{CH}_{2} \mathrm{Cl}_{2}$ was added and the organic layer was then washed 2 times with $10 \mathrm{~mL}$ of saturated aqueous $\mathrm{NaHCO}_{3}$, and 1 time with $10 \mathrm{~mL}$ of saturated aqueous $\mathrm{NaCl}$. The organic layer was dried over $\mathrm{Na}_{2} \mathrm{SO}_{4}$ and concentrated in vacuo, the residue was purified by flash column chromatography on silica gel (EtOAc/n-Hexane $=1: 4)$ to obtain the desired isoxazolo[4,5c]pyridazine 28.

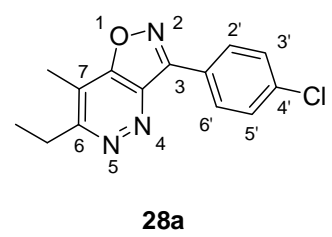

\section{3-(4-Chlorophenyl)-6-ethyl-7-methyl-isoxazolo[4,5-c]pyridazine (28a)}

Beige solid, $167 \mathrm{mg}$ (61\% yield); mp $189-192{ }^{\circ} \mathrm{C}$; Data for 28a: ${ }^{1} \mathrm{H}$ NMR (600 MHz, DMSO-d 6$) \delta 8.55$ (d, $J=8.7 \mathrm{~Hz}, 2 \mathrm{H}, \mathrm{H} 2^{\prime}, \mathrm{H}^{\prime}$ ), 7.78 (d, $J=8.7 \mathrm{~Hz}, 2 \mathrm{H}, \mathrm{H} 3$ ', H5'), 3.20 (q, $J=7.5 \mathrm{~Hz}, 2 \mathrm{H}, \mathrm{CH}_{2}$ ), 2.60 (s, 3H, $\left.\mathrm{CH}_{3}\right), 1.37$ (t, $\left.J=7.5 \mathrm{~Hz}, 3 \mathrm{H}, \mathrm{CH}_{3}\right) ;{ }^{13} \mathrm{C} \mathrm{NMR}\left(150 \mathrm{MHz}, \mathrm{DMSO}-\mathrm{d}_{6}\right) \delta 162.3$ (C), $156.2(\mathrm{C} 3)$, 
154.4 (C6), 144.6 (C1'), 136.9 (C4'), 130.0 (C2', C6’), 129.8 (C3', C5'), 125.2 (C7), 117.3 (C), 25.8 $\left(\mathrm{CH}_{2}\right), 13.3\left(\mathrm{CH}_{3}\right), 10.4\left(\mathrm{CH}_{3}\right)$; HRMS calcd for $\mathrm{C}_{14} \mathrm{H}_{12} \mathrm{ClN}_{3} \mathrm{O}[\mathrm{M}+\mathrm{H}]^{+} 274.0742$, found 274.0743.

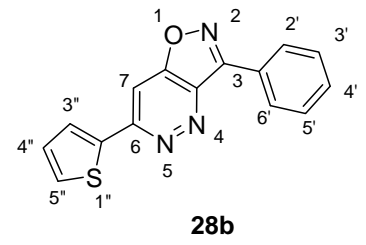

\section{3-Phenyl-6-(thiophen-2-yl)-isoxazolo[4,5-c]pyridazine (28b)}

Orange solid, $128 \mathrm{mg}$ (46\% yield); mp 208-211 ${ }^{\circ} \mathrm{C}$; Data for 28b: ${ }^{1} \mathrm{H}$ NMR (600 MHz, DMSO-d6) $\delta$ 8.80 (s, 1H, H7), 8.52-8.54 (m, 2H, H2', H6'), 8.15 (d, J = 3.7 Hz, 1H, H5”), 7.88 (d, J = 5.0 Hz, 1H, H3”), 7.71-7.72 (m, 3H, H3', H4', H5'), 7.32 (t, $J=5.0 \mathrm{~Hz}, 1 \mathrm{H}, \mathrm{H} 4$ ”); ${ }^{13} \mathrm{C}$ NMR (150 MHz, DMSOd6) $\delta 156.7$ (C), 155.1 (C3), 153.0 (C6), 145.9 (C2”), 139.6 (C1'), 132.1 (C4'), 131.4 (C3”), 129.5 (C2', C6'), 128.9 (C4”), 128.7 (C5”), 128.3 (C3', C5'), 125.9 (C7), 101.8 (C); HRMS calcd for $\mathrm{C}_{15} \mathrm{H}_{9} \mathrm{~N}_{3} \mathrm{OS}[\mathrm{M}+\mathrm{H}]^{+} 280.0539$, found 280.0543 .

\section{Acknowledgments}

The IWT (Agentschap voor Innovatie door Wetenschap en Technologie) and the Galapagos company are acknowledged for providing a PhD-scholarship (Baekeland-project 90704). We are grateful to Prof. Jef Rozenski for recording HRMS spectrums, Luc Baudemprez for NMR experiments and Mrs. Chantal Biernaux for excellent editorial help. We are indebted to Dr. Samia and Dr. Dumbre for helpful discussions.

Supporting Information Available: ${ }^{1} \mathrm{H}$ NMR and ${ }^{13} \mathrm{C}$ NMR spectrums of compounds (10, 15, 16, 18, 19, 21, 22-28), as well as LC/MS analysis of compounds (24b-j, 25a-f, 26a-d, 27a-h, 28a-b). This material is available free of charge via the internet at http://pubs.acs.org.

\section{References}

1 Ritchie, T. J.; Macdonald, S. J. F.; Peace, S.; Pickett, S. D.; Luscombe, C. N. Med. Chem. Commun. 2012, 3, $1062-$ 1069.

2 Wermuth, C. G. Med. Chem. Commun. 2011, 2, 935-941.

3 (a) Boger, D. L. Chem. Rev. 1986, 86, 781-794. (b) Boger, D. L.; Patel, M. Prog. Heterocycl. Chem. 1989, 1, 30 84. (c) Sauer, J. In Comprehensive Heterocyclic Chemistry II; Pergamon: London, 1996; Vol. 6; pp 901-955. (d) Bourguignon, J. J.; Oumouch, S.; Schmitt, M. Curr. Org. Chem. 2006, 10, 277-295. 
4 Wermuth, C. G.; Schlewer, G.; Bourguignon, J.-J.; Maghioros, G.; Bouchet, M.-J.; Moire, C.; Kan, J.-P.; Worms, P.; Biziere, K. J. Med. Chem. 1989, 32, 528-537.

5 Volonterio, A.; Moisan, L.; Rebek, J., Jr Org. Lett. 2007, 9, 3733-3736.

6 Isabel, E.; Powell, D. A.; Black, W. C.; Chan, C.-C.; Crane, S.; Gordon, R.; Guay, J.; Guiral, S.; Huang, Z.; Robichaud, J.; Skorey, K.; Tawa, P.; Xu, L.; Zhang, L.; Oballa, R. Bioorg. Med. Chem. Lett. 2011, 21, 479-483.

7 Wan, Z.; Hall, A.; Jin, Y.; Xiang, J.-N.; Yang, E.; Eatherton, A.; Smith, B.; Yang, G.; Yu, H.; Wang, J.; Ye, L.; Lau, L.-F.; Yang, T.; Mitchell, W.; Cai, W.; Zhang, X.; Sang, Y.; Wang, Y.; Tong, Z.; Cheng, Z.; Hussain, I.; Elliott, J. D.; Matsuoka, Y. Bioorg. Med. Chem. Lett. 2011, 21, 4016-4019.

8 Gleave, R. J.; Beswick, P. J.; Brown, A. J.; Giblin, G. M. P.; Goldsmith, P.; Haslam, C. P.; Mitchell, W. L.; Nicholson, N. H.; Page, L. W.; Patel, S.; Roomans, S.; Slingsby, B. P.; Swarbrick, M. E. Bioorg. Med. Chem. Lett. 2010, 20, 465-468.

9 Asano, T.; Yamazaki, H.; Kasahara, C.; Kubota, H.; Kontani, T.; Harayama, Y.; Ohno, K.; Mizuhara, H.; Yokomoto, M.; Misumi, K.; Kinoshita, T.; Ohta, M.; Takeuchi, M. J. Med. Chem. 2012, 55, 7772-7785.

10 Ellis, G. P. In Synthesis of Fused Heterocycles; John Wiley and Sons Inc.: New York, 1987; pp $226-239$.

11 Grey, R.; Pierce, A. C.; Bemis, G. W.; Jacobs, M. D.; Moody, C. S.; Jajoo, R.; Mohal, N.; Green, J. Bioorg. Med. Chem. Lett. 2009, 19, 3019-3022.

12 Miyamoto, N.; Oguro, Y.; Takagi, T.; Iwata, H.; Miki, H.; Hori, A.; Imamura, S. Bioorg. Med. Chem. 2012, 20, 7051-7058.

13 Hu, E.; Kunz, R. K.; Rumfelt, S.; Chen, N.; Bürli, R.; Li, C.; Andrews, K. L.; Zhang, J.; Chmait, S.; Kogan, J.; Lindstrom, J.; Hitchcock, S. A.; Treanor, J. Bioorg. Med. Chem. Lett. 2012, 22, 2262-2265.

14 Dyck, B.; Markison, S.; Zhao, L.; Tamiya, J.; Grey, J.; Rowbottom, M. W.; Zhang, M.; Vickers, T.; Sorensen, K.; Norton, C.; Wen, J.; Heise, C. E.; Saunders, J.; Conlon, P.; Madan, A.; Schwarz, D.; Goodfellow, V. S. J. Med. Chem. 2006, 49, 3753-3756.

15 (a) Bel Abed, H.; Mammoliti, O.; Van Lommen, G.; Herdewijn, P. Tetrahedron Lett. 2013, 54, 2612-2614. (b) Bel Abed, H.; Mammoliti, O.; Van Lommen, G.; Herdewijn, P. Tetrahedron Lett. 2012, 53, 6489-6491.

16 (a) Galiullina, S. V.; Zakharova, V. M.; Kantin, G. P.; Nikolaev, V. A. Zh. Org. Khim. 2007, 43, 607-614. (b) Supurgibekov, M. B.; Hennig, L.; Schulze, B.; Nikolaev, V. A. Zh. Org. Khim. 2008, 44, 1840-1843. (c) Supurgibekov, M. B.; Yanyuk, N. S.; Nikolaev, V. A. Zh. Org. Khim. 2011, 47, 1252-1255.

17 (a) Draper, T. L.; Bailey, T. R. J. Org. Chem. 1995, 60, 748-750. (b) Helm, M. D.; Moore, J. E.; Plant, A.; Harrity, J. P. A. Angew. Chem. Int. Ed. 2005, 44, 3889-3892. (c) Yang, X.; Knochel, P. Org. Lett. 2006, 9, 1941-1943. (d) Hamasaki, A.; Ducray, R.; Boger, D. L. J. Org. Chem. 2006, 71, 185-193.

18 (a) Zalesov, V. V.; Vyaznikova, N. G.; Andreichikov, Y. S. Zh. Org. Khim. 1996, 32, 705-709. (b) Zalesov, V. V.; Vyaznikova, N. G.; Andreichikov, Y. S. Zh. Org. Khim. 1996, 32, 735-740. (c) Aliev, Z. G.; Vyaznikova N. G.; Zalesov, V. V.; Kataev, S. S.; Andreichikov, Y. S.; Atovmyan, L. O. Russ. Chem. Bull. 1997, 46, 2142-2145. (d) Kutkovaya, N. V.; Vyaznikova, N. G.; Zalesov, V. V. Zh. Org. Khim. 2003, 39, 1713-1717. (e) Kutkovaya, N. V.; Pulina, N. A.; Zalesov, V. V. Zh. Org. Khim. 2004, 40, 1078-1081.

19 (a) Regitz, M.; Maas, G. Diazo Compounds: Properties and Synthesis; Academic Press: London, 1986, 65-198. (b) Padwa, A.; Weingarten, M. D. Chem. Rev. 1996, 96, 223-269. (c) Doyle, M. P.; McKervey, M. A.; Ye, T. Modern Catalytic Methods for Organic Synthesis with Diazo Compounds; Wiley: New York, 1998. (d) Davies, H. M. L.; 
Beckwith, R. E. J. Chem. Rev. 2003, 103, 2861-2903. (e) Zhang, Z.; Wang, J. Tetrahedron 2008, 64, 6577-6605. (f) Maas, G. Angew. Chem. Int. Ed. 2009, 48, 8186-8195. (g) Xu, X.; Hu, W.-H.; Doyle, M. P. Angew. Chem., Int. Ed. 2011, 50, 6392-6395. (h) Zhou, L.; Doyle, M. P. Org. Lett. 2010, 12, 796-799. (i) Yu, L.; Zhang, Y.; Jee, N.; Doyle, M. P. Org. Lett. 2008, 10, 1605-1608. (j) Doyle, M. P.; Kundu, K.; Russell, A. E. Org. Lett. 2005, 7, 5171 5174. (k) Deng, G.; Tian, X.; Qu, Z.; Wang, J. Angew. Chem. Int. Ed. 2002, 41, 2773-2776. (1) Liao, M.; Dong, S.; Deng, G.; Wang, J. Tetrahedron Lett. 2006, 47, 4537-4540. (m) Zhao, Y.; Wang, J. Synlett 2005, 2886-2892. (n) Dong, C.; Mo, F.; Wang, J. J. Org. Chem. 2008, 73, 1971-1974. (o) Dong, C.; Deng, G.; Wang, J. J. Org. Chem. 2006, 71, 5560-5564. (p) Padwa, A.; Zhang, Z. J.; Zhi, L. J. Org. Chem. 2000, 65, 5223-5232.

20 Calter, M. A.; Zhu, C. J. Org. Chem. 1999, 64, 1415-1419.

21 Frigerio, M.; Santagostino, M.; Sputore, S. J. Org. Chem. 1999, 64, 4537-4538.

22 Ashby, J.; Styles, J. A.; Paton, D. Br. J. Cancer 1978, 38, 418-427.

23 Fluck, E. Top. Curr. Chem. 1973, 35.

24 (a) Beck, A. K.; Hoekstra, M. S.; Seebach, D. Tetrahedron Lett. 1977, 18, 1187-1190. (b) Tang, Q.; Sen, S. E. Tetrahedron Lett. 1998, 39, 2249-2252. (c) Katritzky, A. R.; Pastor, A. J. Org. Chem. 2000, 65, 3679-3682. (d) Le Roux, C.; Mandrou, S.; Dubac, J. J. Org. Chem. 1996, 61, 3885-3887. (e) Wiles, C.; Watts, P.; Haswell, S. J.; Pombo-Villar, E. Tetrahedron Lett. 2002, 43, 2945-2948. (f) Kel'in, A. V. Curr. Org. Chem. 2003, 7, 1-21. (g) Ballini, R.; Bartoli, G. Synthesis 1993, 10, 965-967.

25 List, B.; Lerner, R. A.; Barbas III, C. F. J. Am. Chem. Soc. 2000, 122, 2395-2396.

26 Sakthivel, K.; Notz, W.; Bui, T.; Barbas III, C. F. J. Am. Chem. Soc. 2001, 123, 5260-5267.

27 Mukaiyama, T. Angew. Chem., Int. Ed. Engl. 1977, 16, 817-826.

28 Degradation of the crude product 22a (obtained from the titanium aldol reaction between 19a and p-methoxy benzaldehyde) into the enone 23 during the purification by flash chromatography on silica gel.

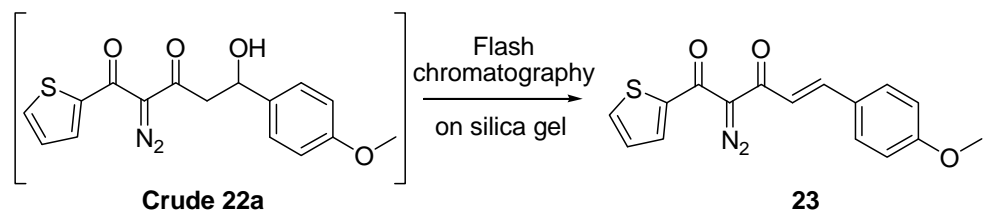

2-Diazo-(4-methoxyphenyl)-1-(thiophen-2-yl)-pent-4-ene-1,3-dione (23)

Yellow solid, $194 \mathrm{mg}\left(62 \%\right.$ yield); $\mathrm{mp} 120-123{ }^{\circ} \mathrm{C}$; Data for 23: ${ }^{1} \mathrm{H}$ NMR $\left(500 \mathrm{MHz}, \mathrm{DMSO}-\mathrm{d}_{6}\right) \delta 8.07(\mathrm{~d}, J=3.9 \mathrm{~Hz}$, $1 \mathrm{H}), 7.97(\mathrm{~d}, J=3.8 \mathrm{~Hz}, 1 \mathrm{H}), 7.56-7.74(\mathrm{~m}, 4 \mathrm{H}), 7.26(\mathrm{t}, J=3.9 \mathrm{~Hz}, 1 \mathrm{H}), 7.02(\mathrm{~d}, J=8.8 \mathrm{~Hz}, 2 \mathrm{H}), 3.82(\mathrm{~s}, 3 \mathrm{H}) ;{ }^{13} \mathrm{C}$ NMR (125 MHz, DMSO-d 6 ) $\delta 181.2,175.6,161.6,142.1,141.8,134.7,132.0,130.6,128.4,126.9,120.5,114.7,82.9$, 55.5 . 


\section{Supporting Information}

\section{Strategy for the synthesis of pyridazine heterocycles and its derivatives}

Hassen Bel Abed, ${ }^{\S}$ Oscar Mammoliti, ${ }^{\mathbb{I}}$ Omprakash Bande, ${ }^{\S}$ Guy Van Lommen ${ }^{\mathbb{I}}$ and Piet Herdewijn ${ }^{\S, *}$

${ }^{\S}$ Rega Institute for Medical Research, Laboratory of Medicinal Chemistry, Katholieke Universiteit Leuven, Minderbroedersstraat 10, B-3000 Leuven, Belgium

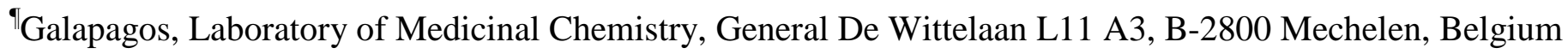

Table of Contents

\section{${ }^{1} \mathrm{H}$ and ${ }^{13} \mathrm{C}$ NMR spectra}

${ }^{1} \mathrm{H}$ and ${ }^{13} \mathrm{C}$ NMR spectra for $\mathbf{1 0}$ S2

${ }^{1} \mathrm{H}$ and ${ }^{13} \mathrm{C}$ NMR spectra for $\mathbf{1 5 a}-\mathbf{b}$ S3-4

${ }^{1} \mathrm{H}$ and ${ }^{13} \mathrm{C}$ NMR spectra for $\mathbf{1 6} \mathbf{a}-\mathbf{b}$ S5-6

${ }^{1} \mathrm{H}$ and ${ }^{13} \mathrm{C}$ NMR spectra for $\mathbf{1 8 a - b}$ S7-8

${ }^{1} \mathrm{H}$ and ${ }^{13} \mathrm{C}$ NMR spectra for $\mathbf{1 9 a - b}$ S9-10

${ }^{1} \mathrm{H}$ and ${ }^{13} \mathrm{C}$ NMR spectra for 21 S11

${ }^{1} \mathrm{H}$ and ${ }^{13} \mathrm{C}$ NMR spectra for $\mathbf{2 2 b}$-j

${ }^{1} \mathrm{H}$ and ${ }^{13} \mathrm{C}$ NMR spectra for $\mathbf{2 3}$

${ }^{1} \mathrm{H}$ and ${ }^{13} \mathrm{C}$ NMR spectra for $\mathbf{2 4 b}-\mathbf{j}$ S22-30

${ }^{1} \mathrm{H}$ and ${ }^{13} \mathrm{C}$ NMR spectra for $\mathbf{2 5 a - f}$ S31-36

${ }^{1} \mathrm{H}$ and ${ }^{13} \mathrm{C}$ NMR spectra for 26a-d S37-40

${ }^{1} \mathrm{H}$ and ${ }^{13} \mathrm{C}$ NMR spectra for $\mathbf{2 7} \mathbf{a}-\mathbf{h}$ S41-48

${ }^{1} \mathrm{H}$ and ${ }^{13} \mathrm{C}$ NMR spectra for $\mathbf{2 8 a}-\mathbf{b}$ S49-50

\section{LC/MS analysis}

LC/MS analysis for $\mathbf{2 4 b}$-j

LC/MS analysis for 25a-f

LC/MS analysis for 26a-d

LC/MS analysis for 27a-h 


$$
\prod_{\substack{\mathrm{N}_{2} \\ 10}}^{O} \mathrm{O}^{-}
$$

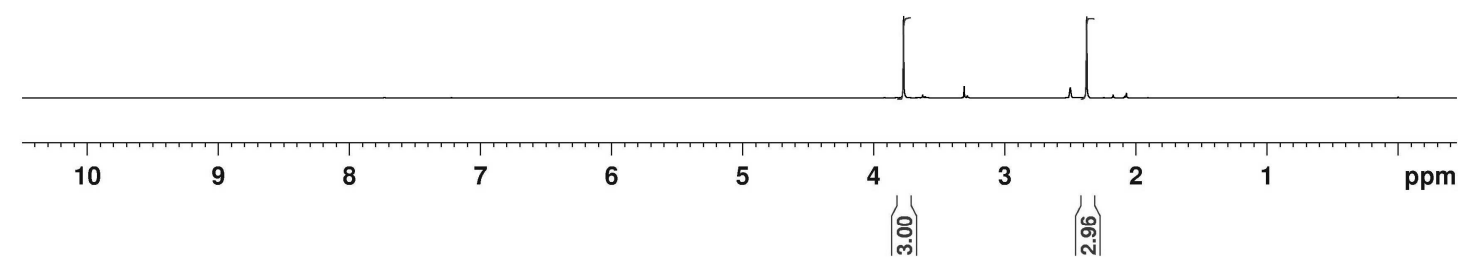

10 carbon spectrum (125 MHz) in DMSO-d6

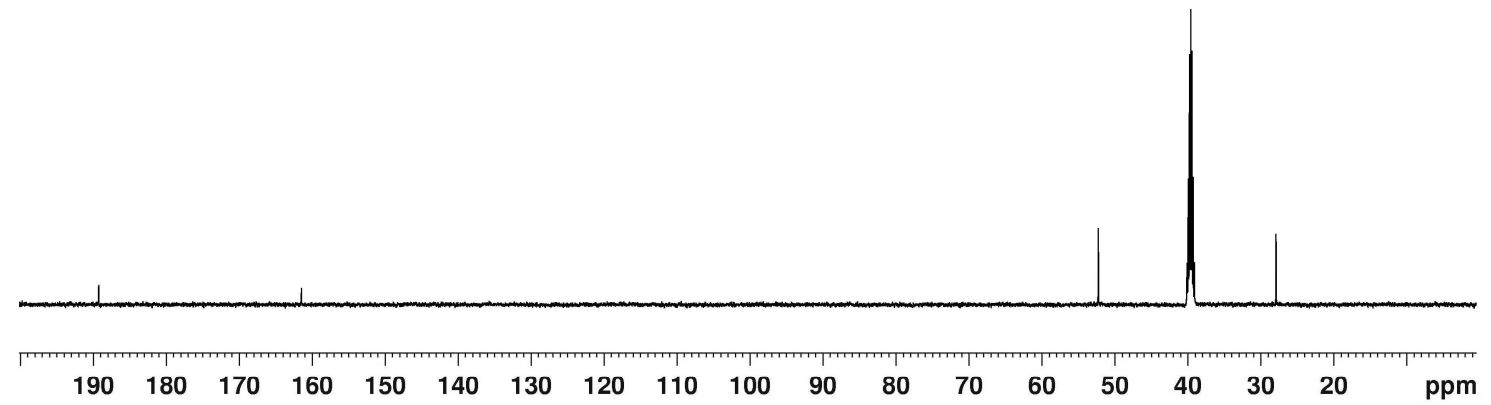




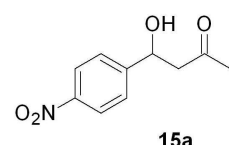

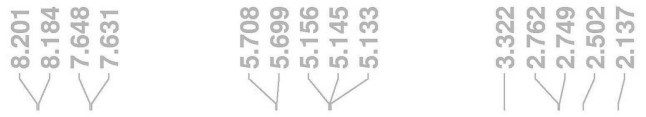

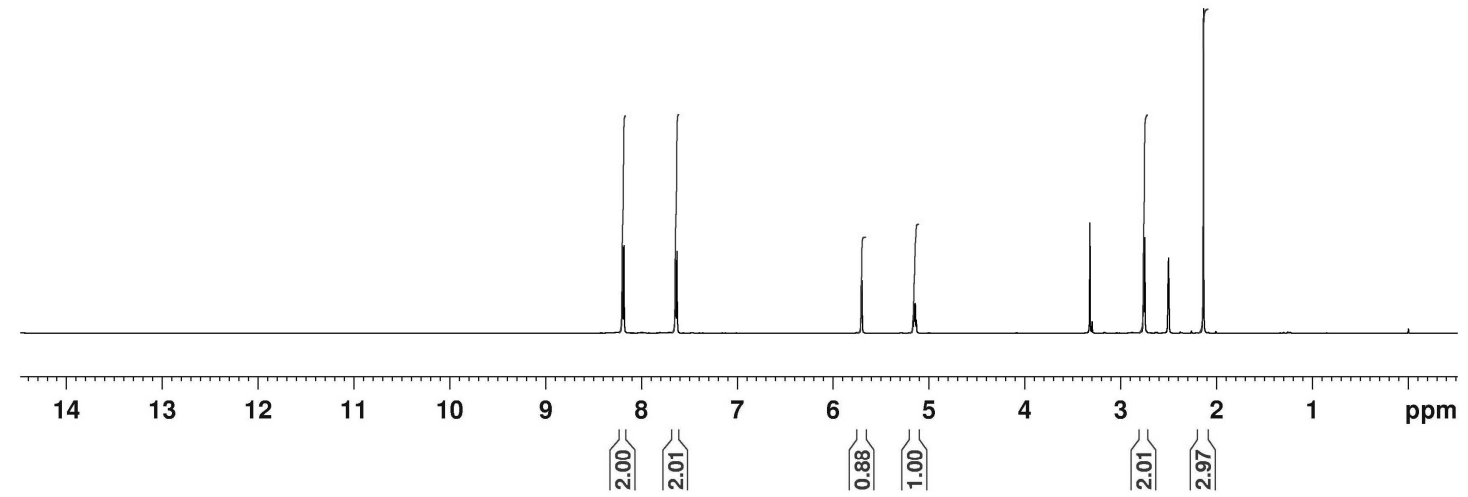

15 a carbon spectrum (125 MHz) in DMSO-d6
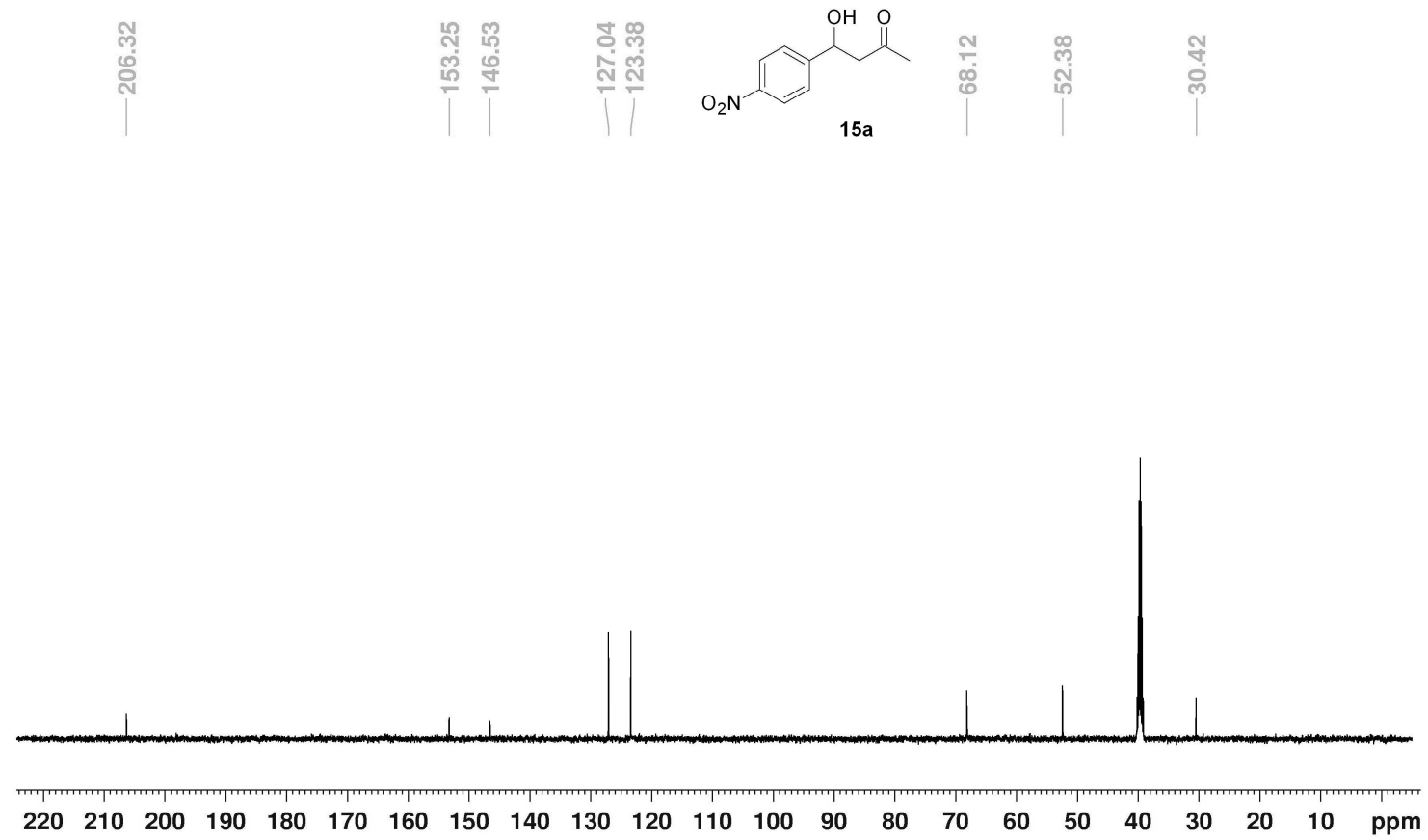
15b proton spectrum ( $500 \mathrm{MHz}$ ) in DMSO-d6
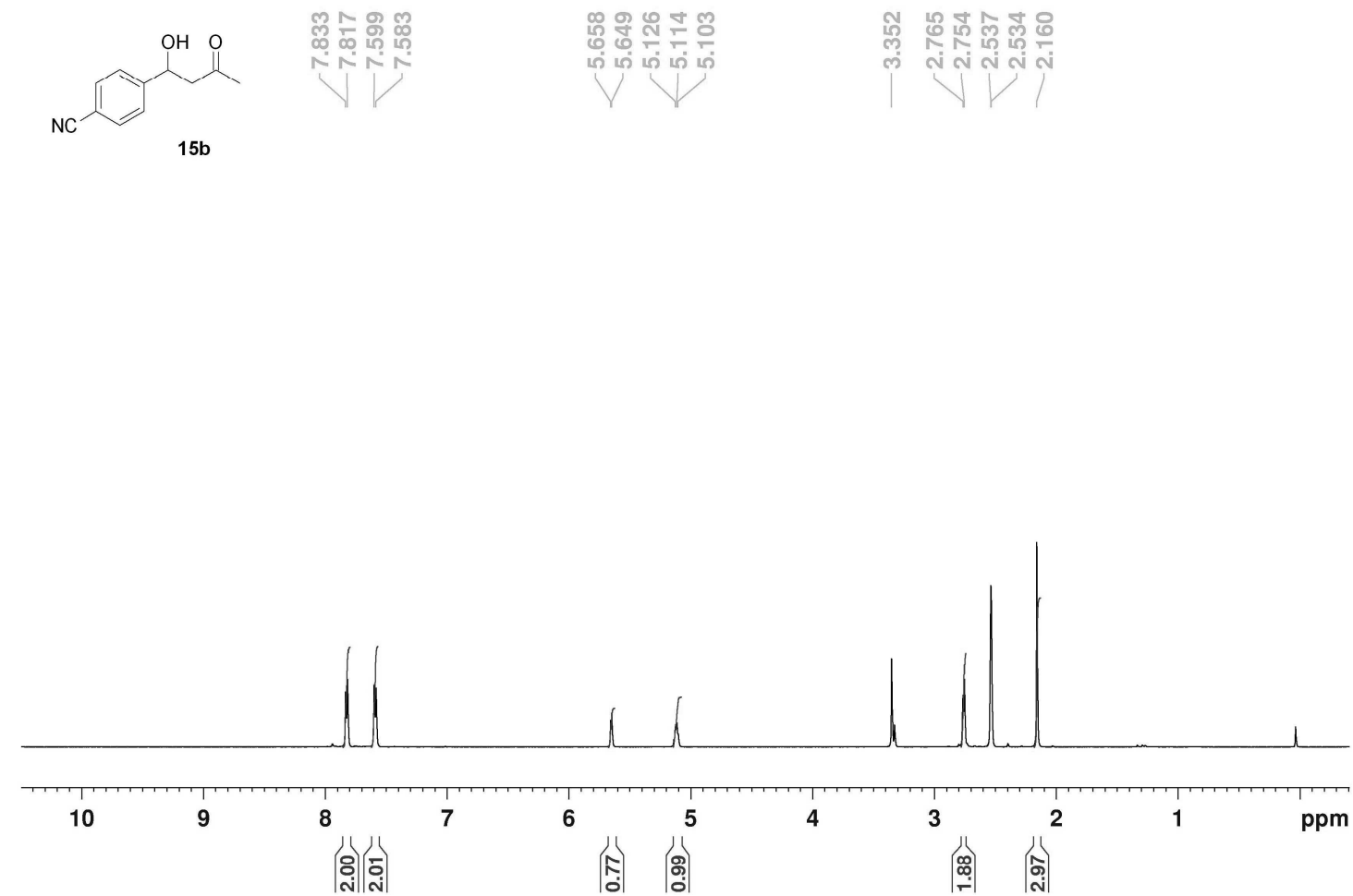

15b carbon spectrum (125 MHz) in DMSO-d6
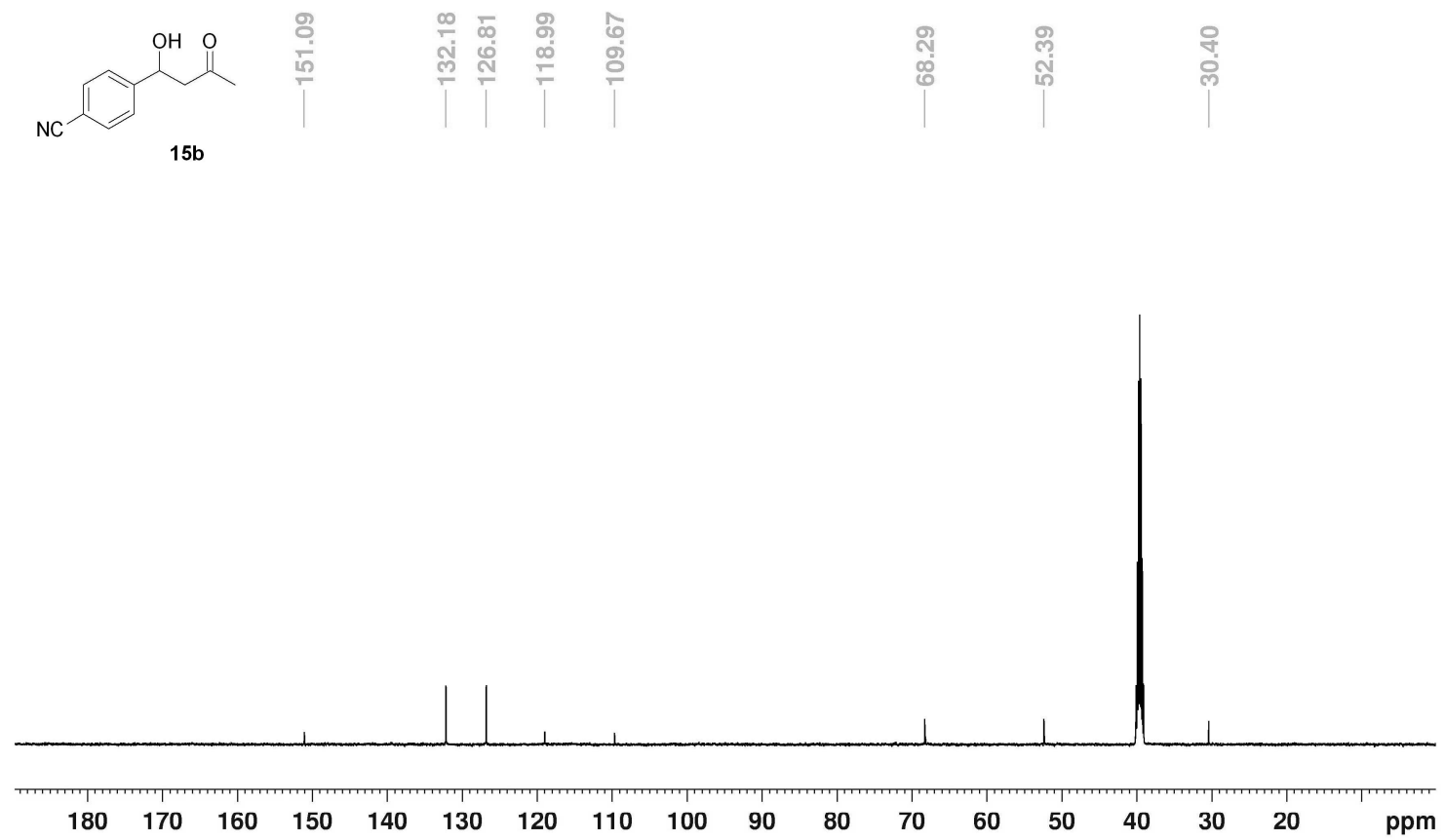


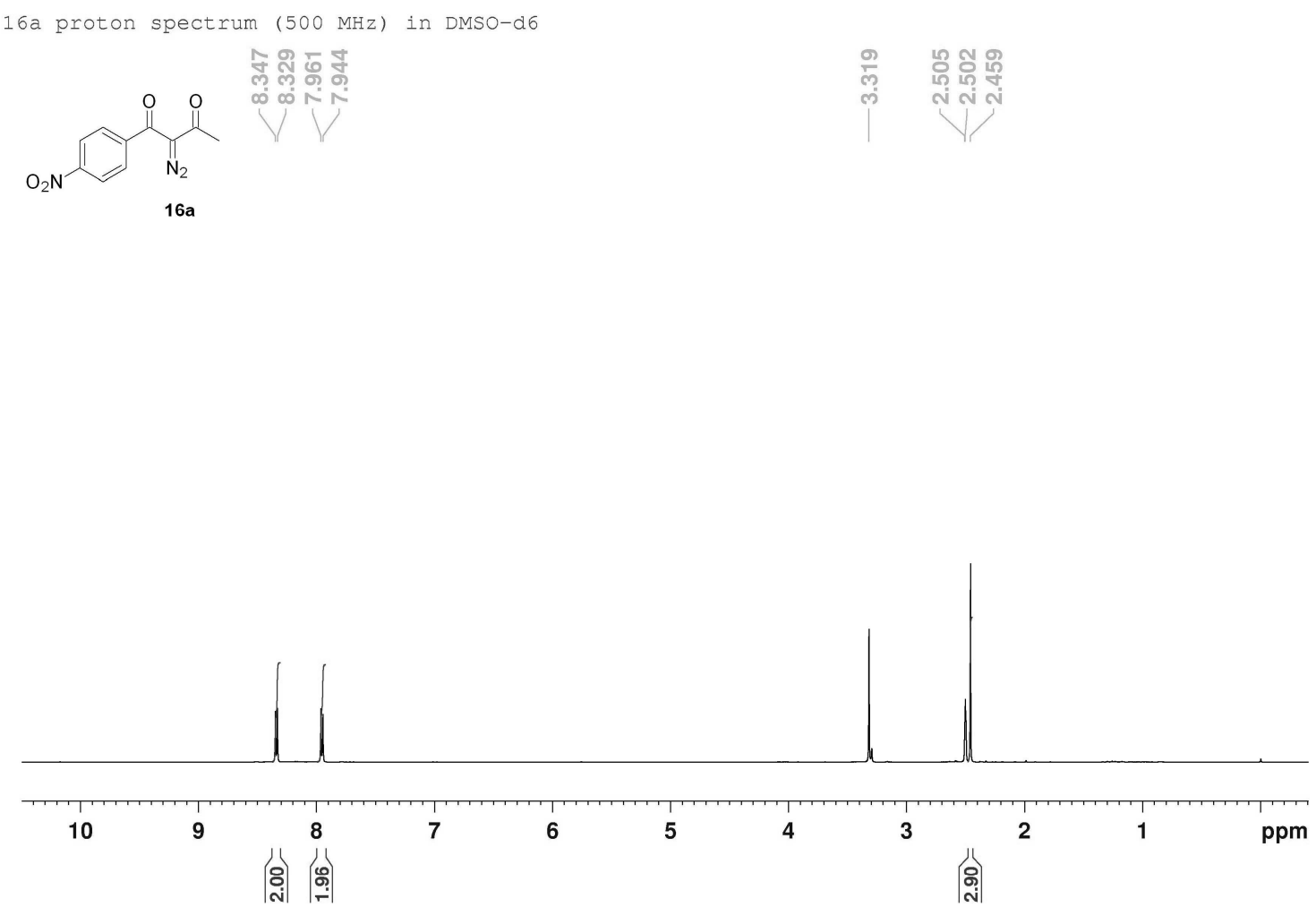

호

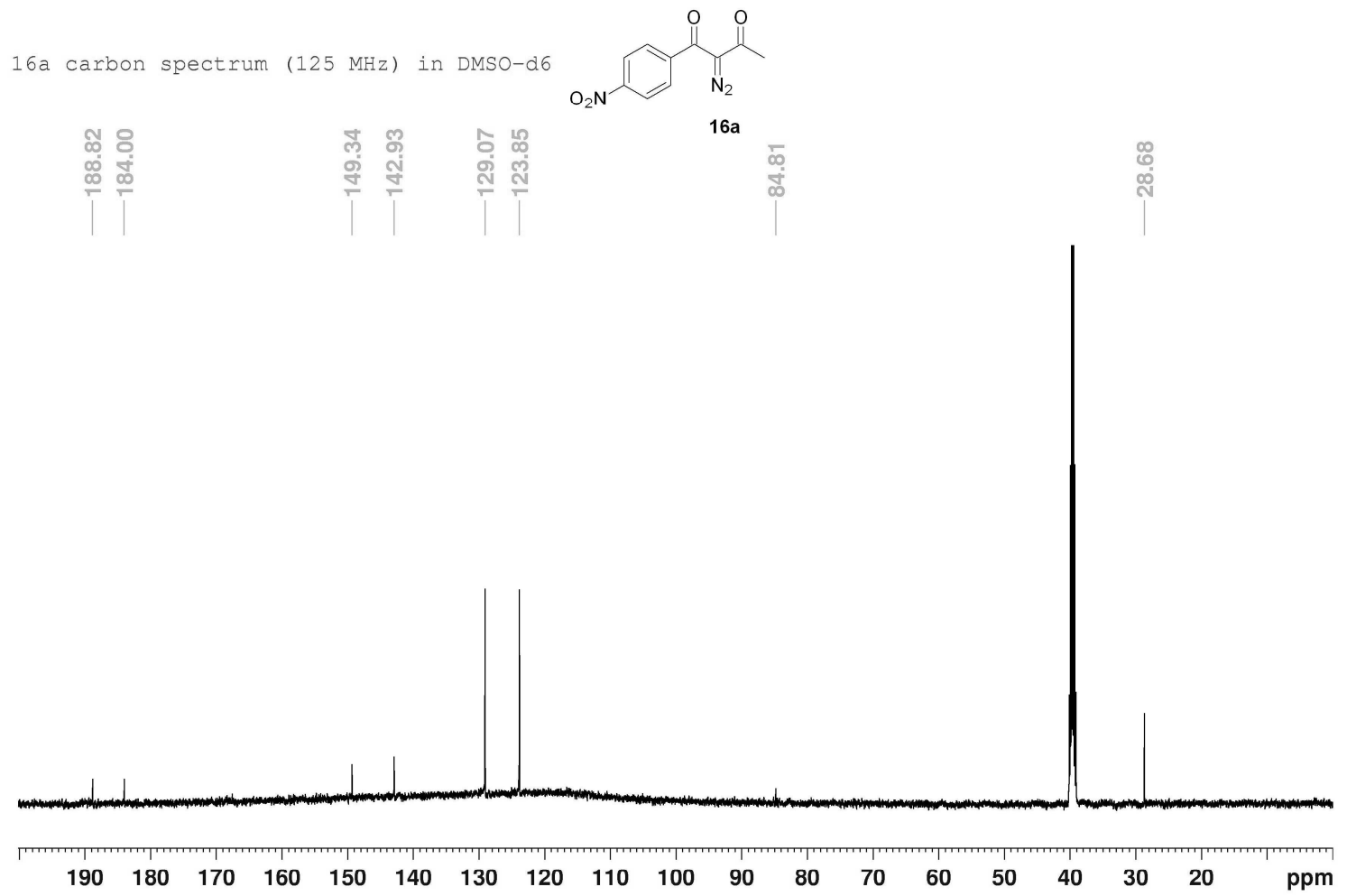


$16 \mathrm{~b}$ proton spectrum ( $500 \mathrm{MHz}$ ) in DMSO-d6

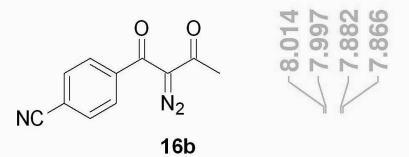

$16 b$

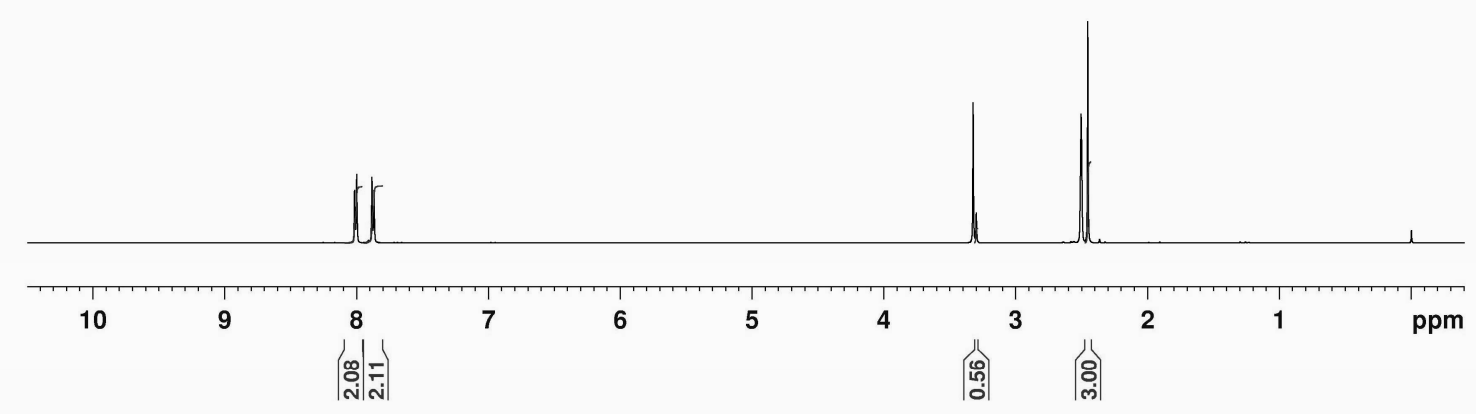

16b carbon spectrum (125 MHz) in DMSO-d6

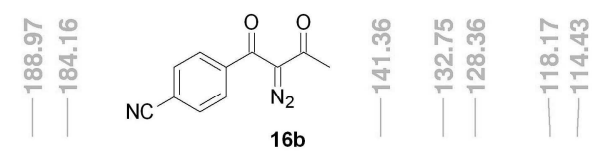

ஜீ

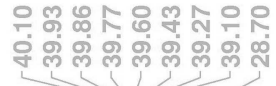

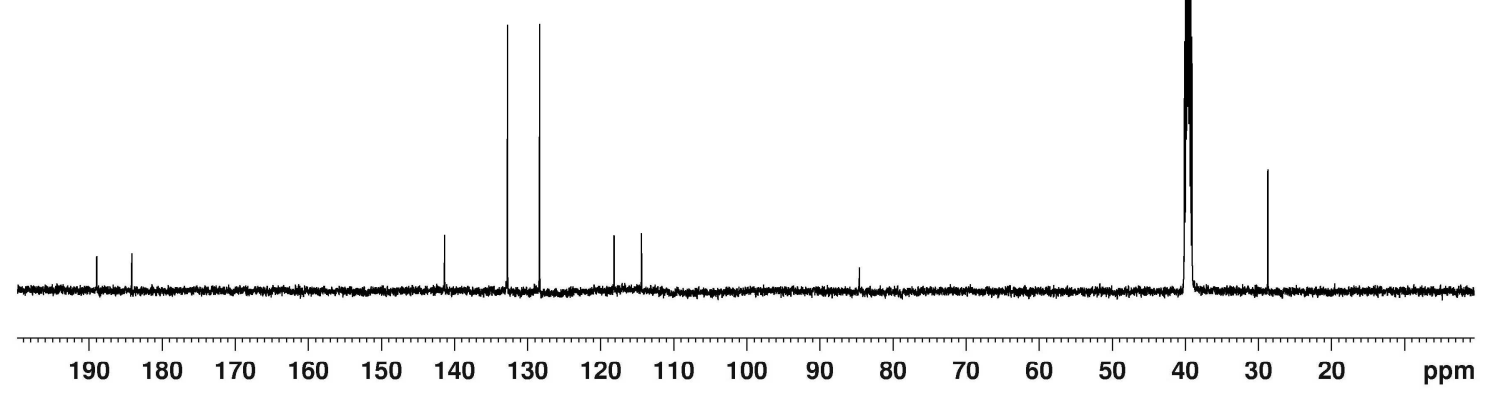


18 a proton spectrum $(500 \mathrm{MHz})$ in DMSO-d6

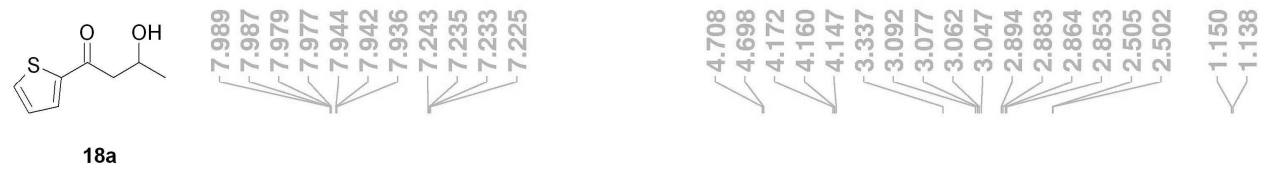

$18 a$

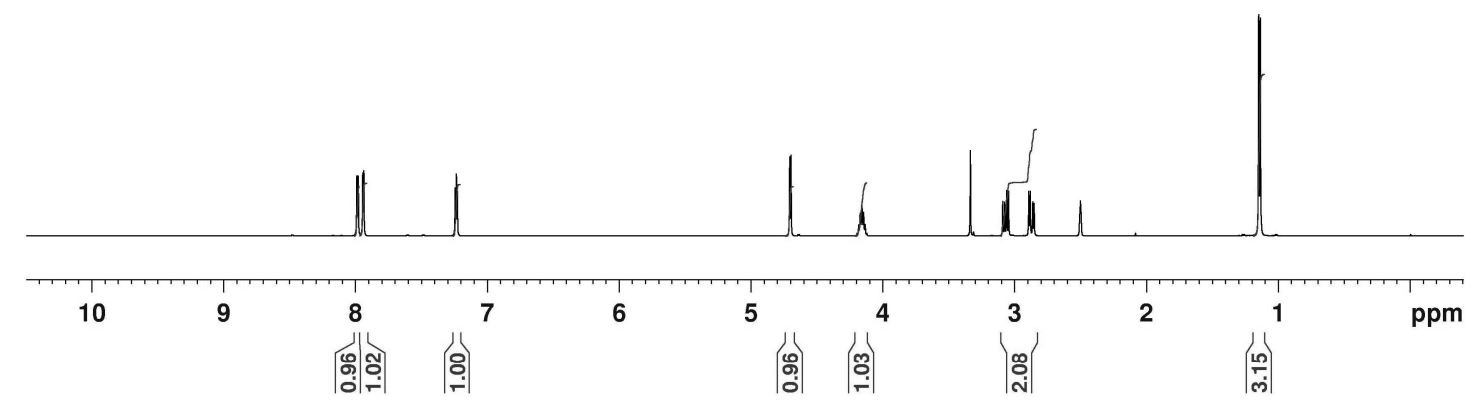

18 a carbon spectrum (125 MHz) in DMSO-d6
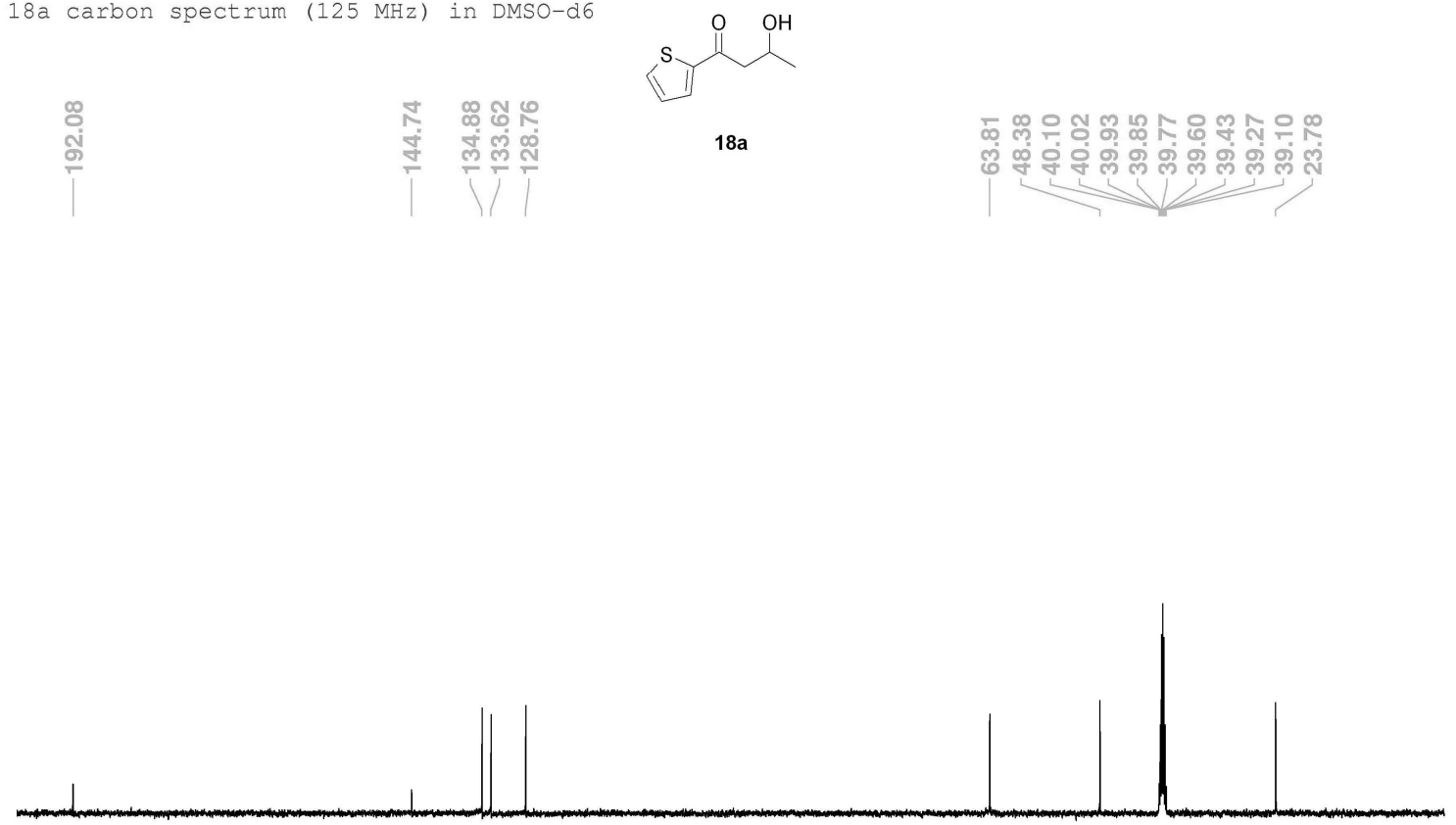

$\begin{array}{lllllllllllllllllll}190 & 180 & 170 & 160 & 150 & 140 & 130 & 120 & 110 & 100 & 90 & 80 & 70 & 60 & 50 & 40 & 30 & 20 & \mathrm{ppm}\end{array}$ 
18b proton spectrum ( $500 \mathrm{MHz})$ in DMSO-d6

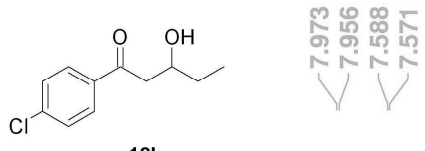

$18 b$

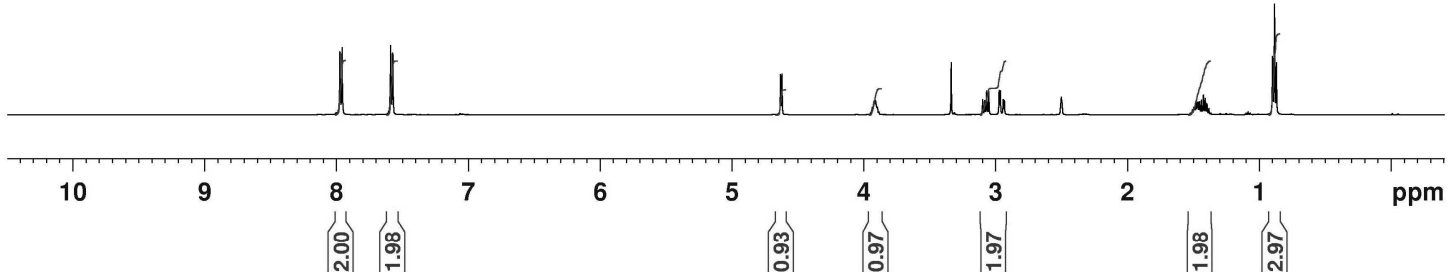

18b carbon spectrum (125 $\mathrm{MHz})$ in DMSO-d6

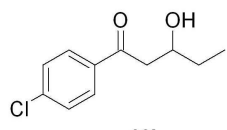

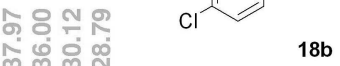

$\begin{array}{llll}8 & 0 & 0 & 8 \\ 0 & 0 & 0 \\ 0 & \text { 1 } & 0\end{array}$

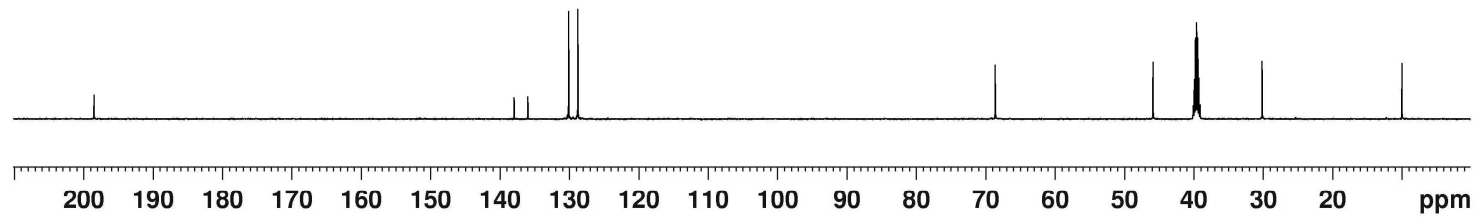




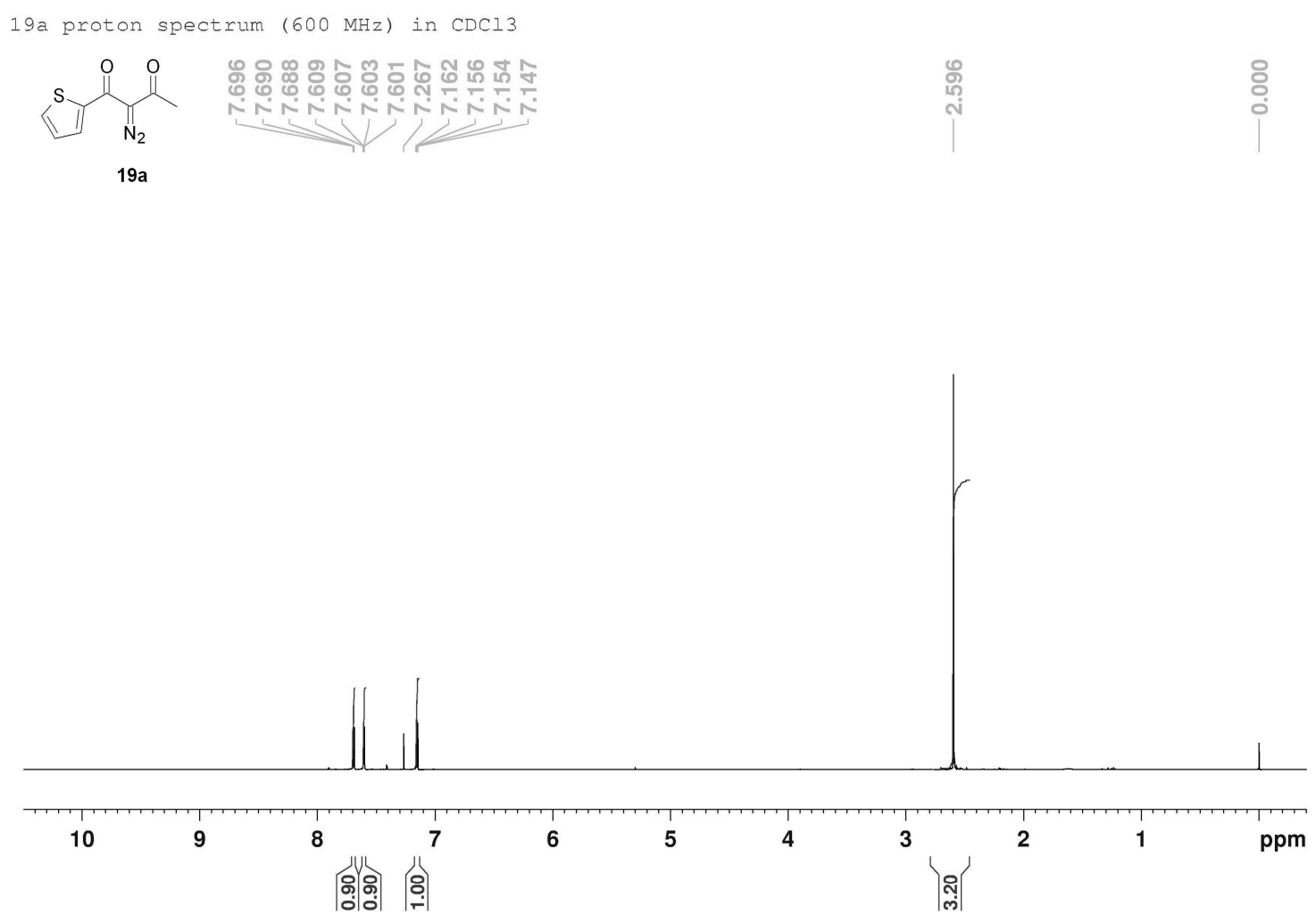

19a carbon spectrum (150 $\mathrm{MHz})$ in $\mathrm{CDCl3}$

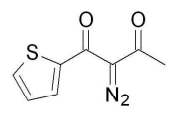

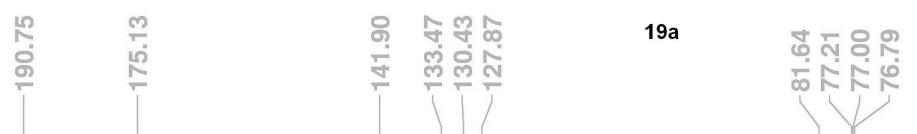

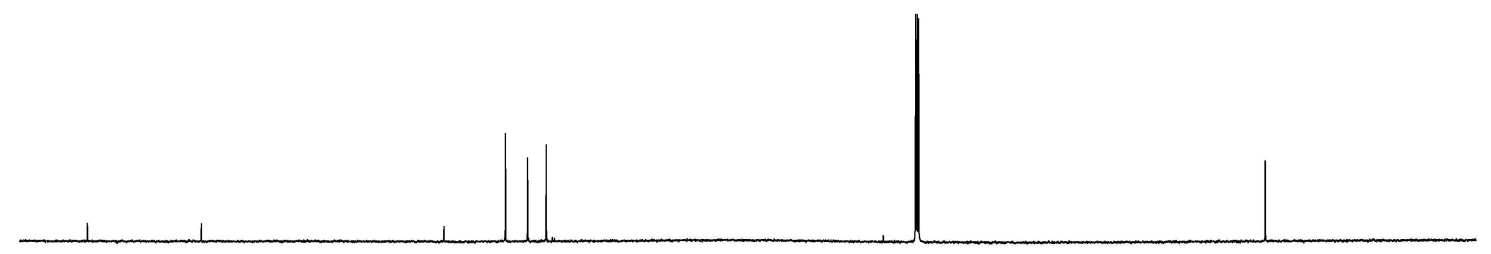

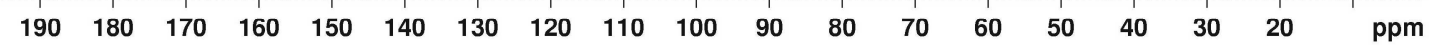


$19 \mathrm{~b}$ proton spectrum $(500 \mathrm{MHz})$ in DMSO-d6
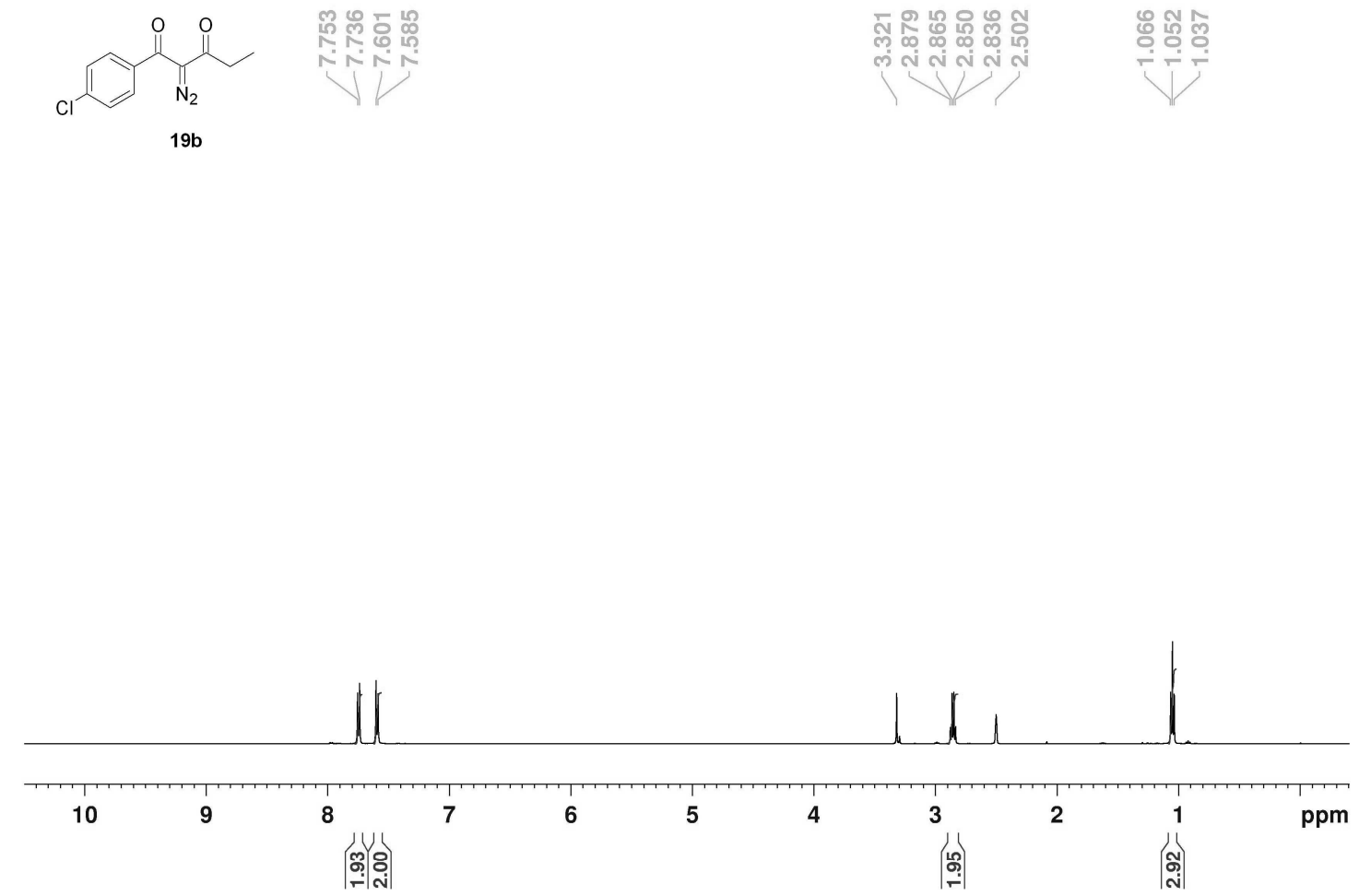

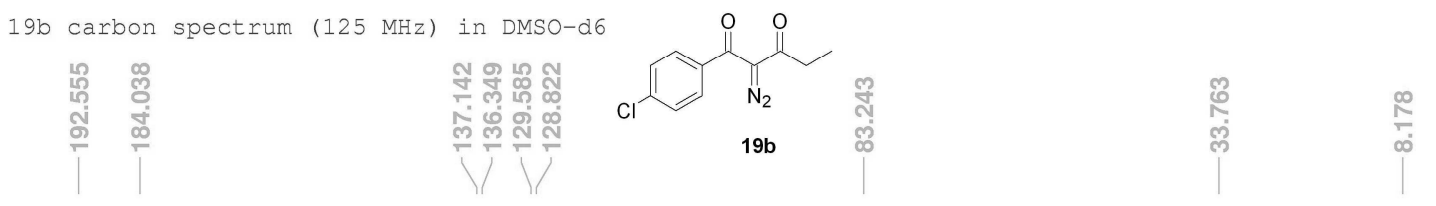

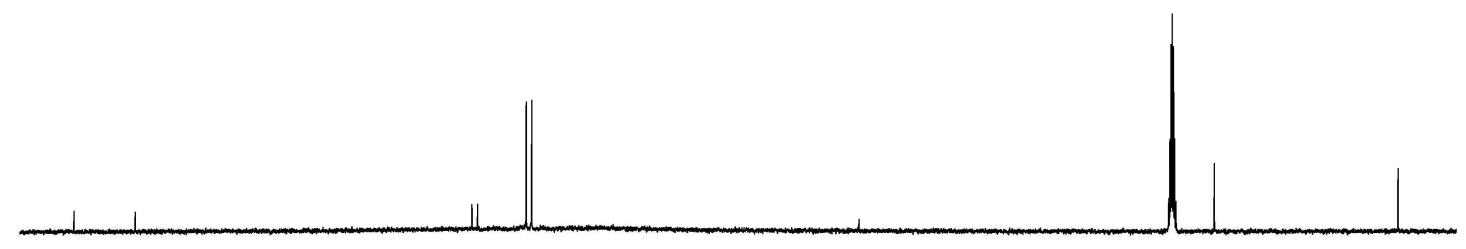

$\begin{array}{lllllllllllllllllllll}190 & 180 & 170 & 160 & 150 & 140 & 130 & 120 & 110 & 100 & 90 & 80 & 70 & 60 & 50 & 40 & 30 & 20 & \mathrm{ppm}\end{array}$ 
21 proton spectrum $(500 \mathrm{MHz})$ in DMSO-d6
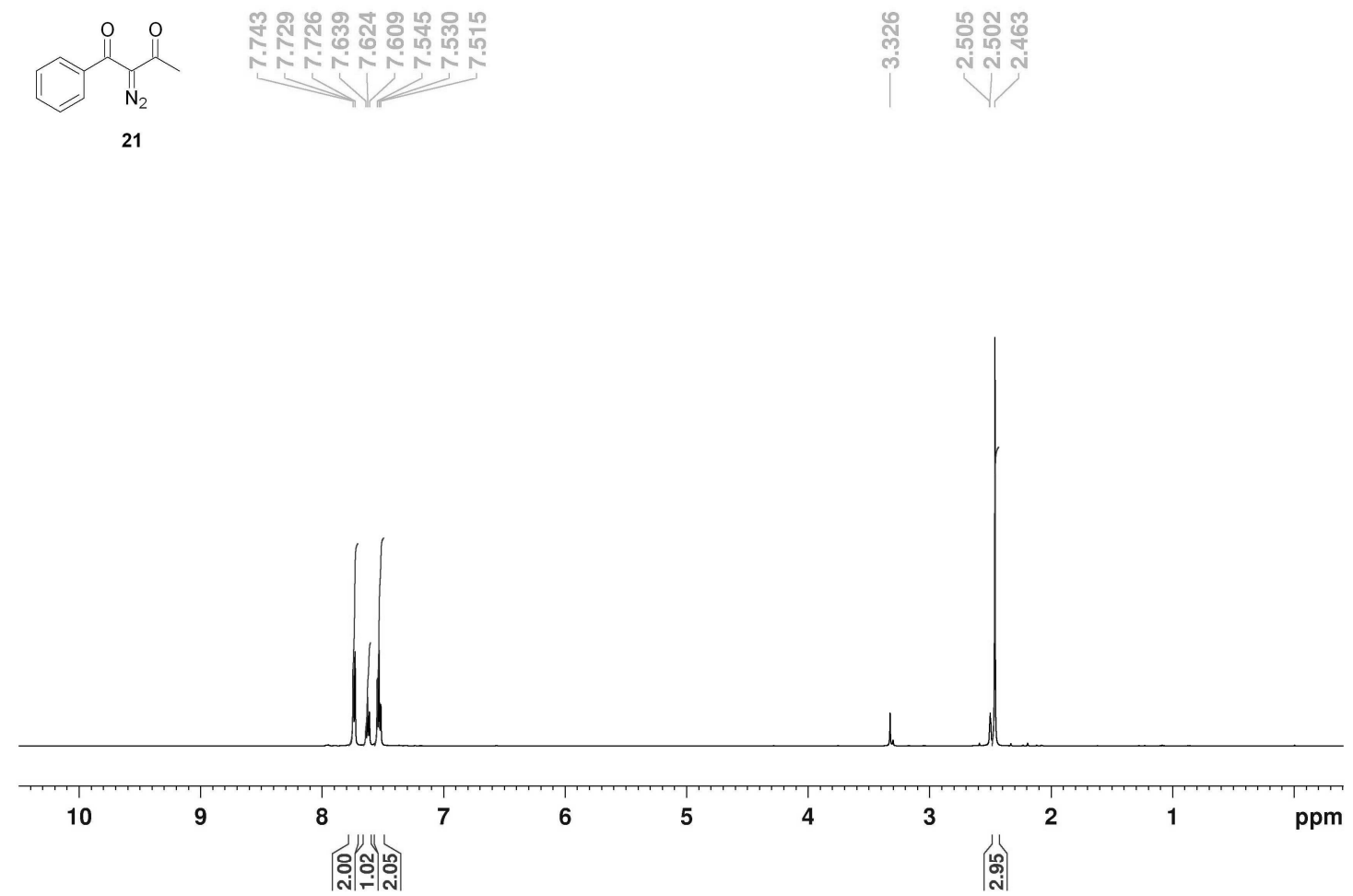

21 carbon spectrum (125 $\mathrm{MHz})$ in DMSO-d6

$\hat{N}$ 。

ณ ฌ

ๆั ำ

लำ ํำ ำ

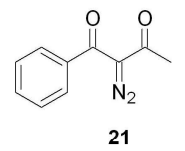

్ֶֻ

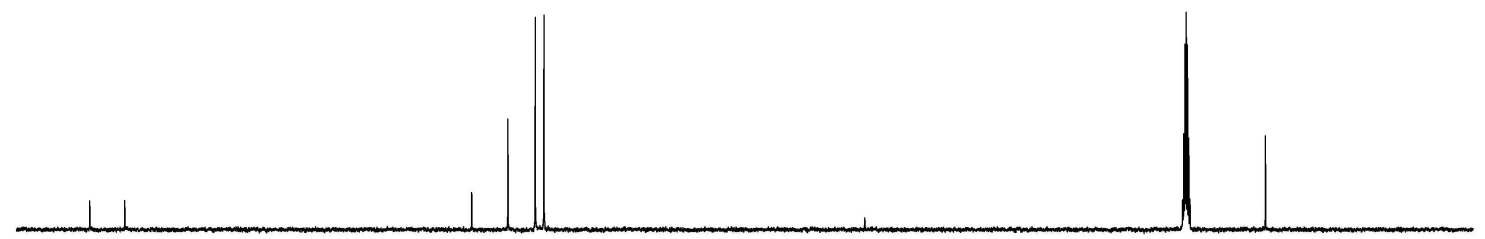

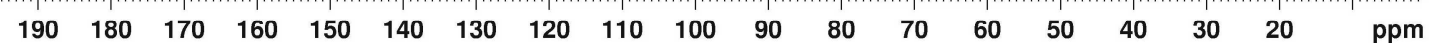


$22 \mathrm{~b}$ proton spectrum (300 $\mathrm{MHz})$ in DMSO-d6

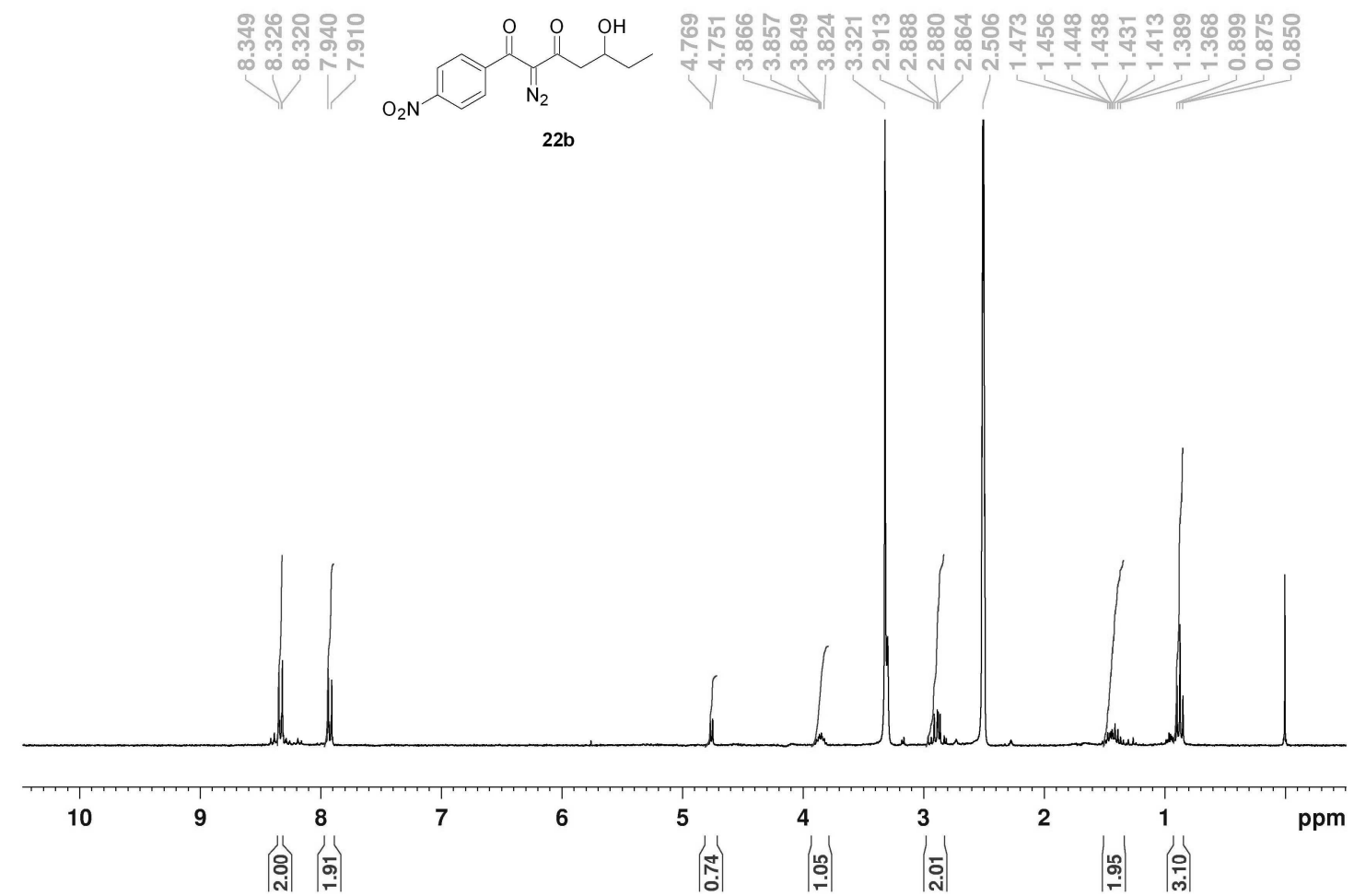

22b carbon spectrum ( $75 \mathrm{MHz}$ ) in DMSO-d6
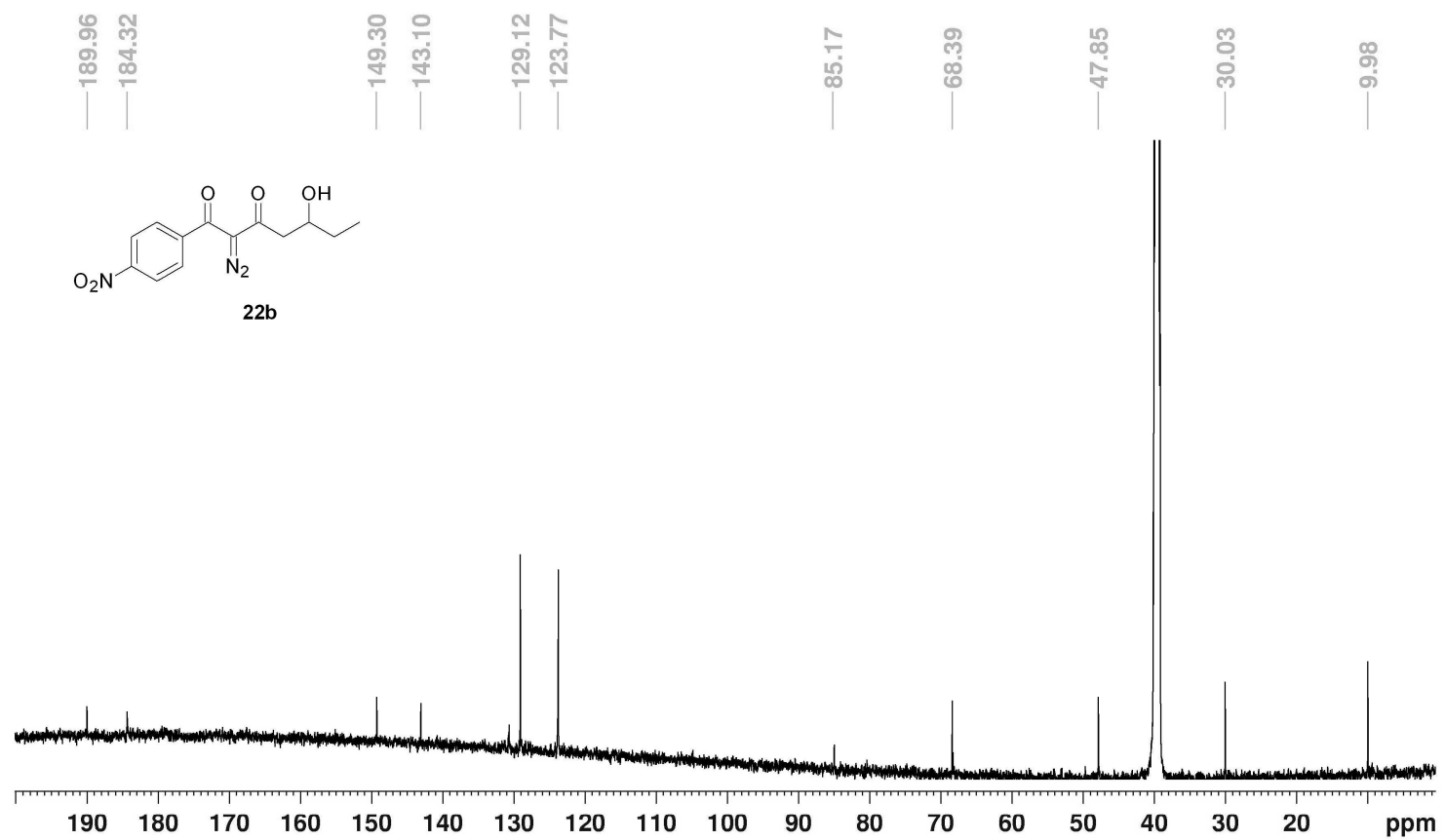
<smiles>CCC(O)CC(=O)C(=N)C(=O)c1ccc(C#N)cc1</smiles>

22c

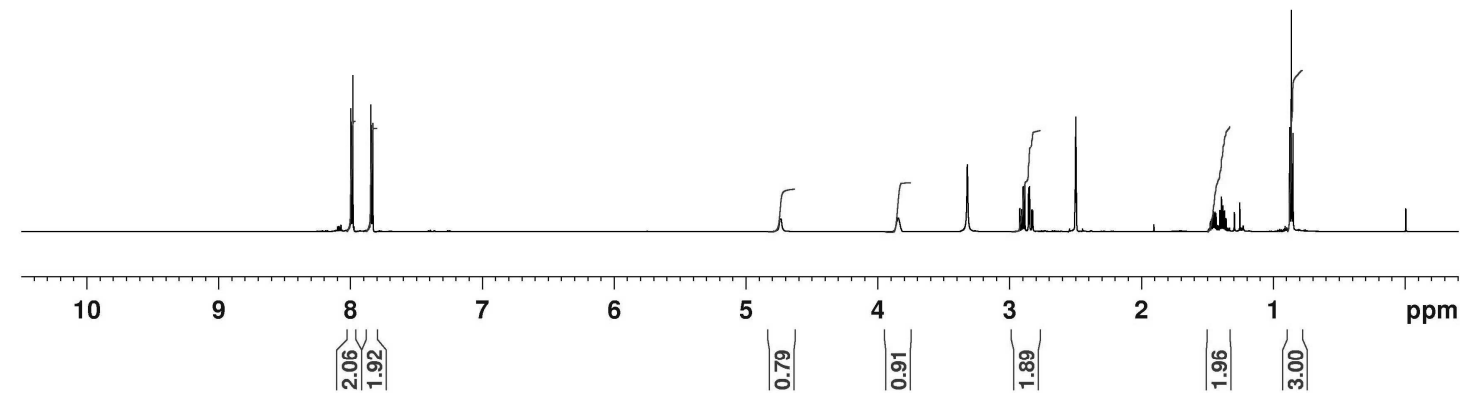

22c carbon spectrum (150 MHz) in DMSO-d6
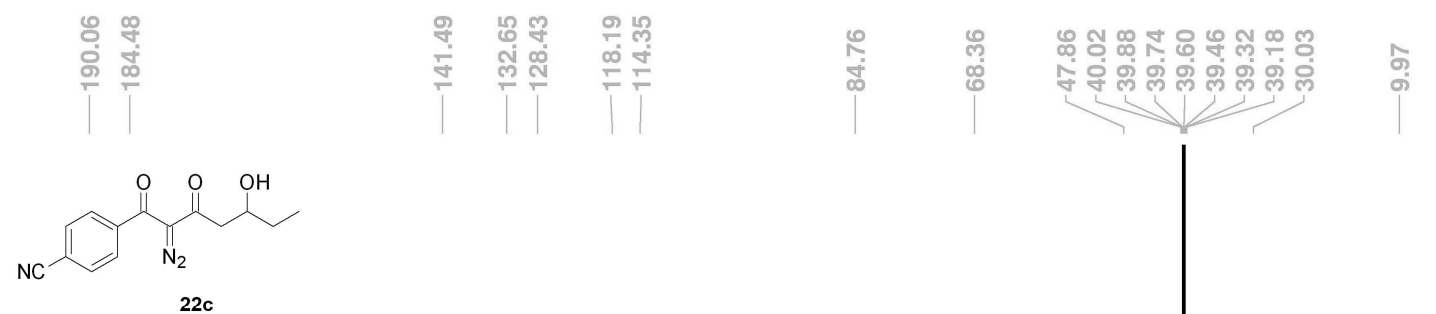

22c

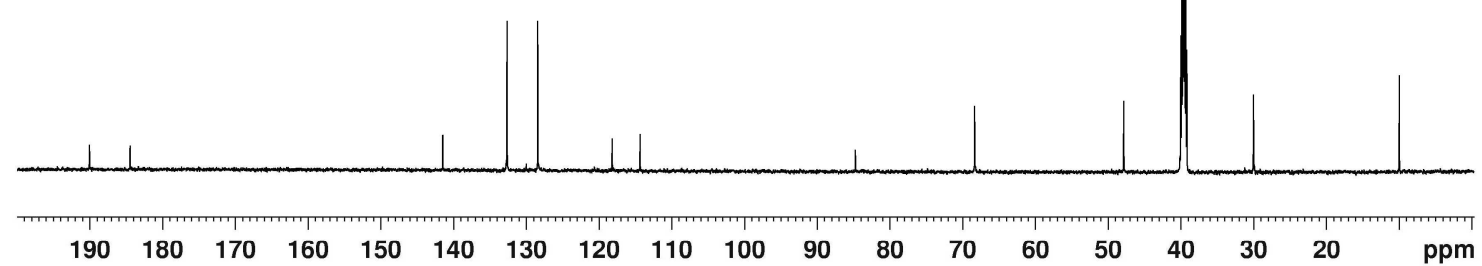




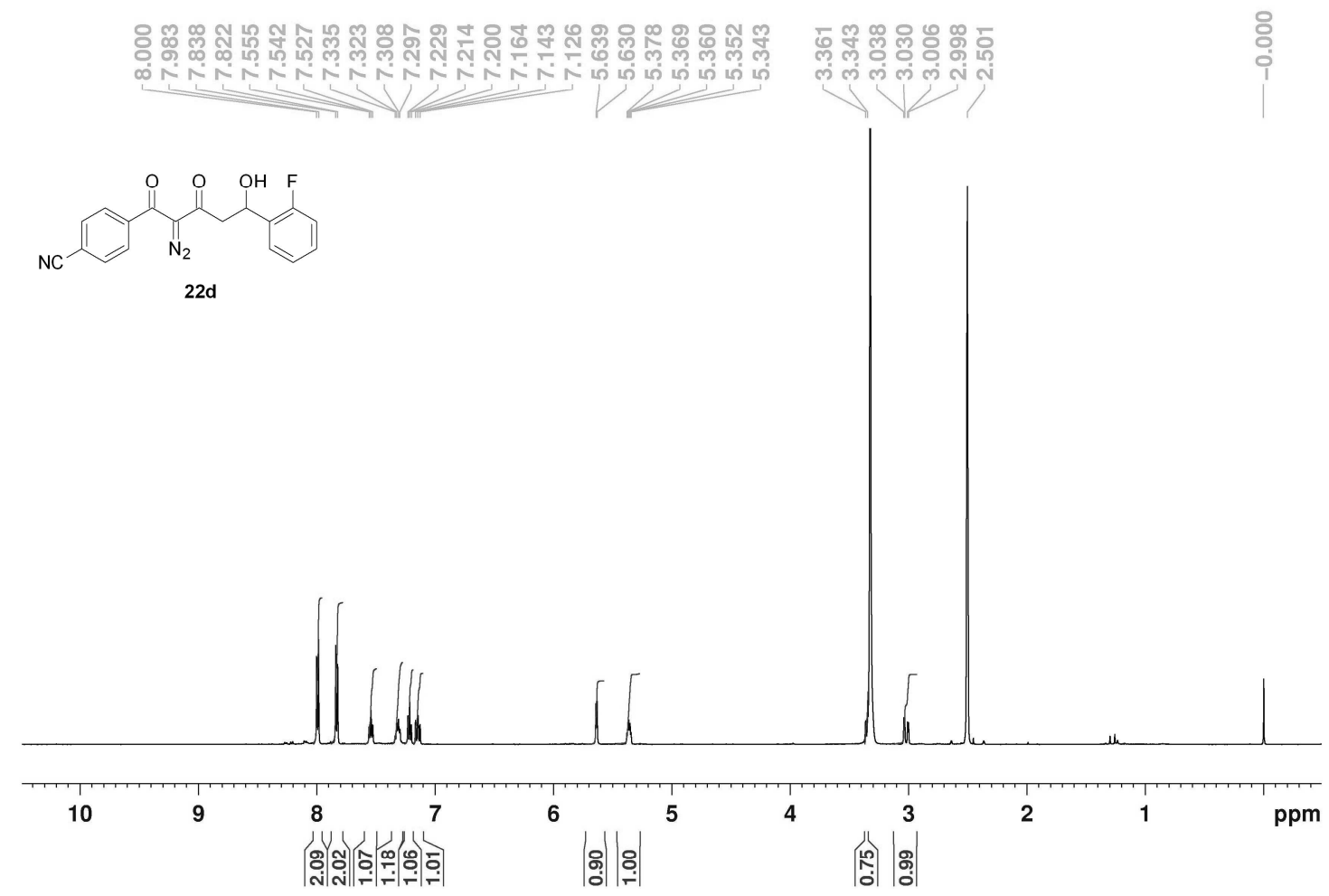

22d carbon spectrum (125 MHz) in DMSO-d6

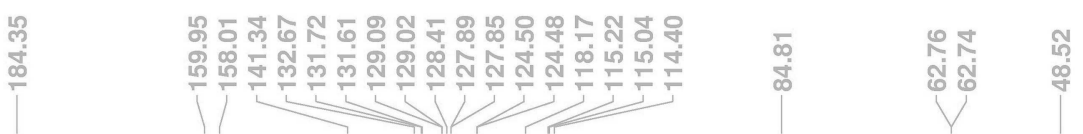
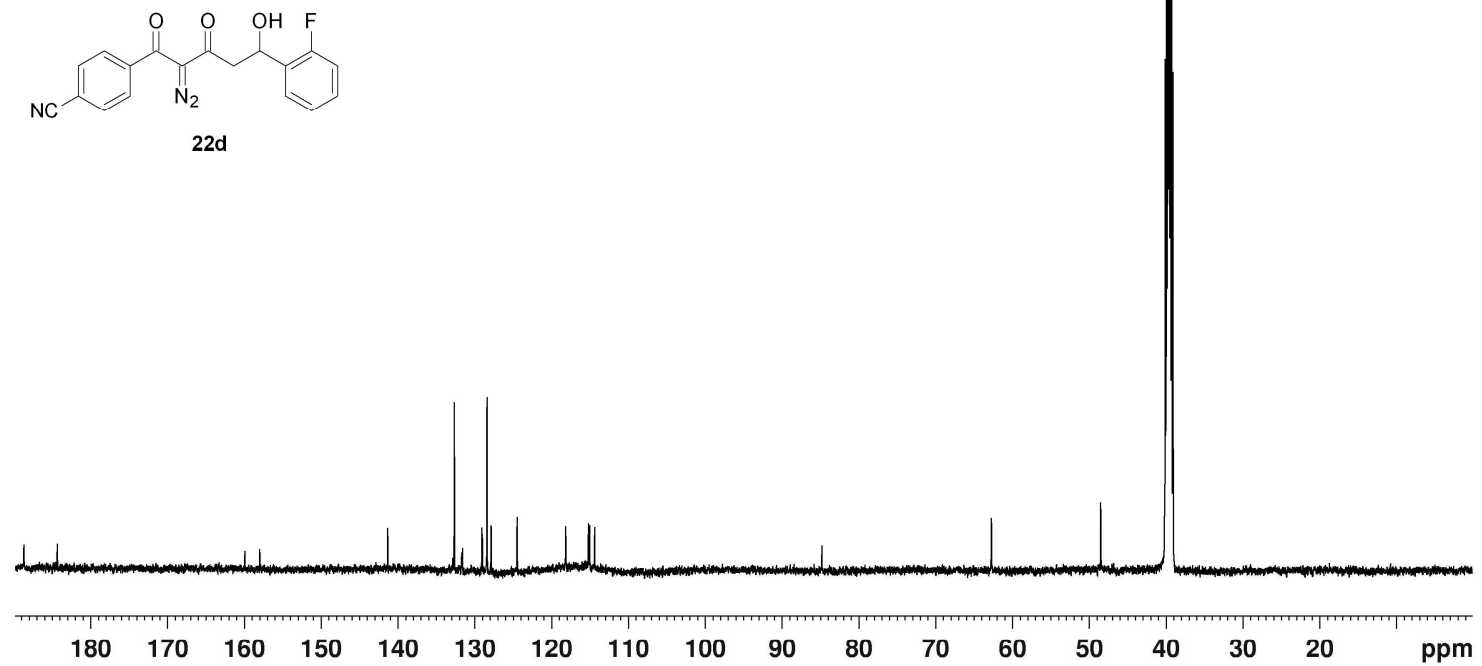


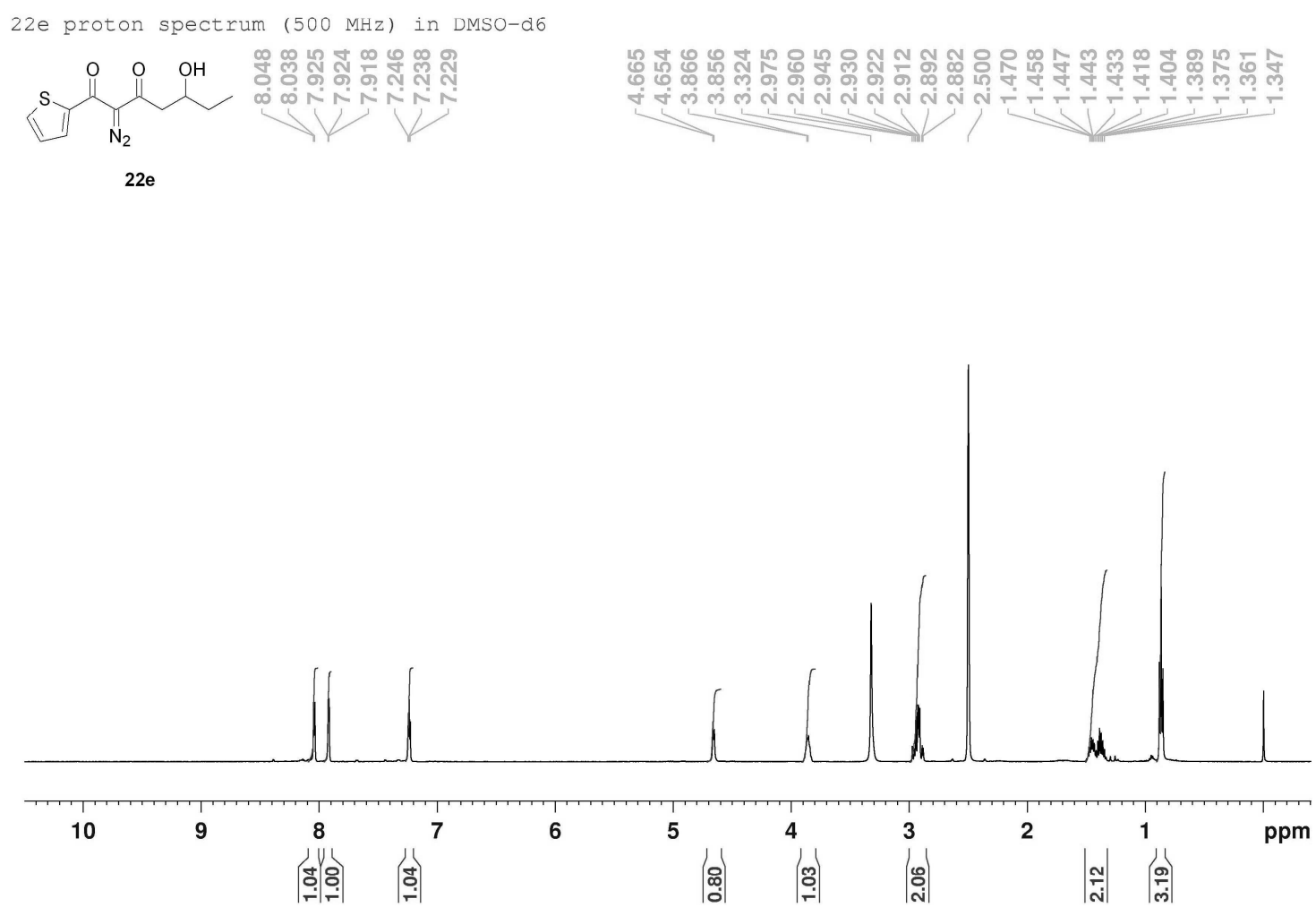

22 e carbon spectrum (125 MHz) in DMSO-d6
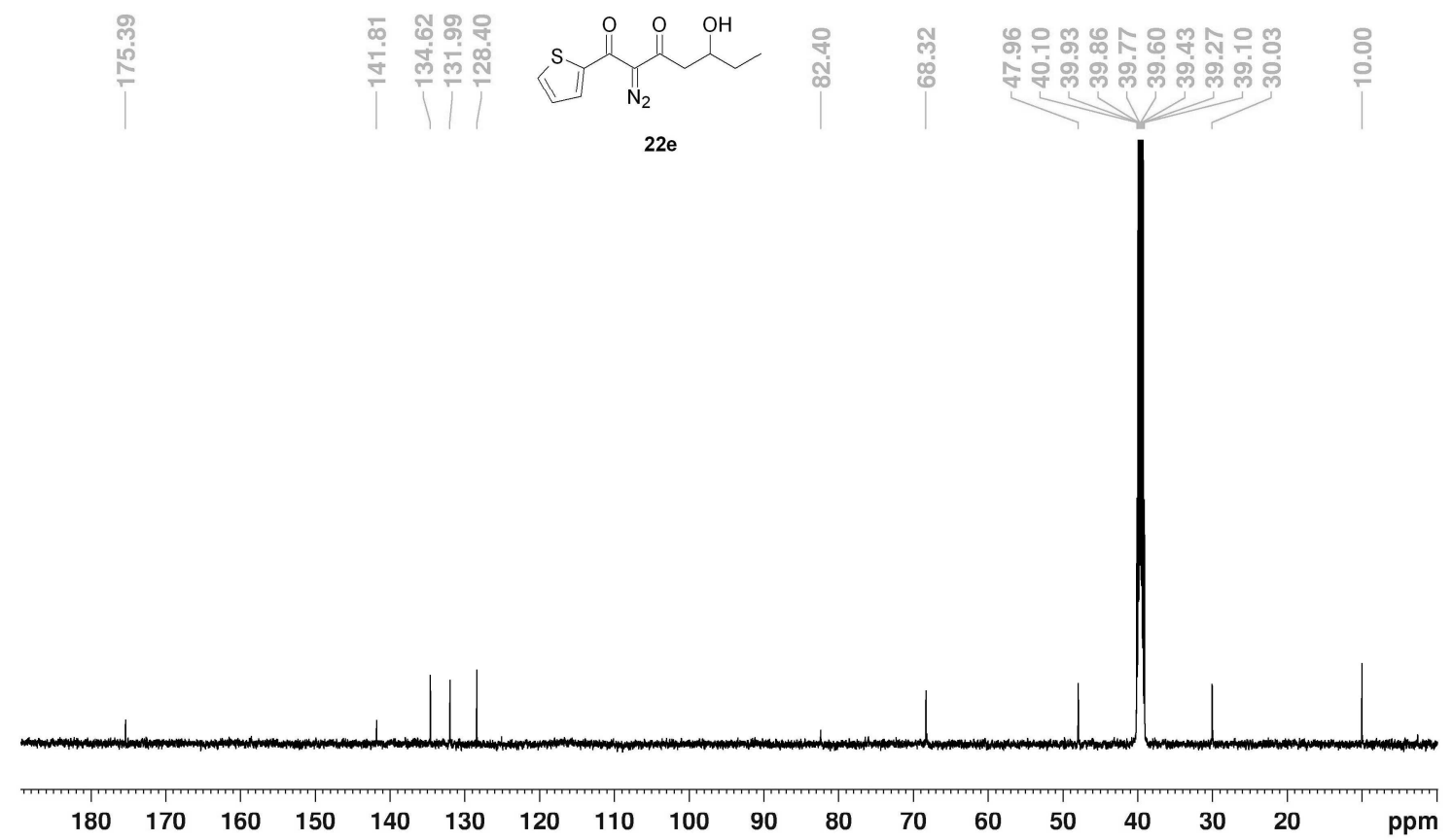


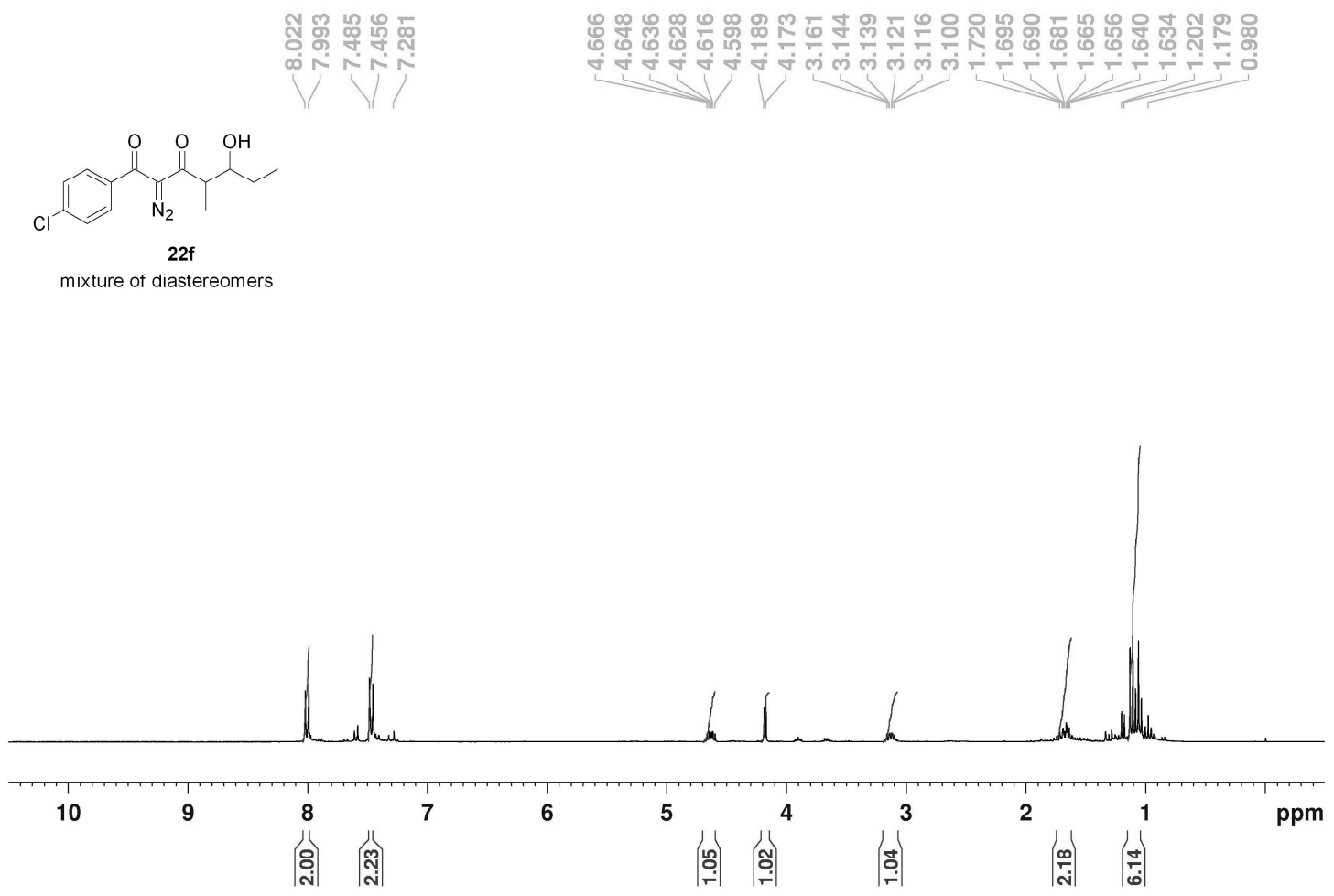

$22 \mathrm{f}$ carbon spectrum $(75 \mathrm{MHz})$ in $\mathrm{CDCl} 3$
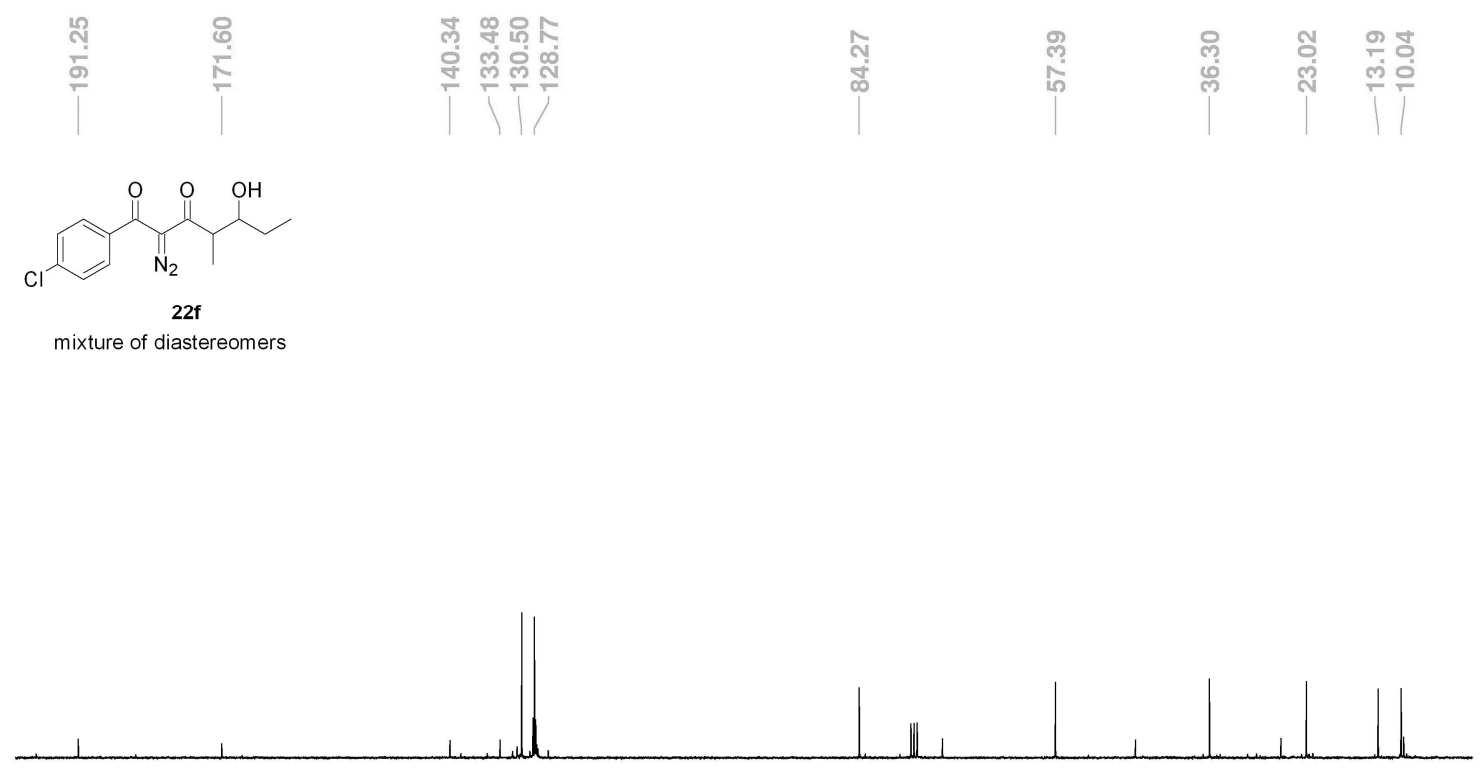

$\begin{array}{lllllllllllllllllll}190 & 180 & 170 & 160 & 150 & 140 & 130 & 120 & 110 & 100 & 90 & 80 & 70 & 60 & 50 & 40 & 30 & 20 & \mathrm{ppm}\end{array}$ 


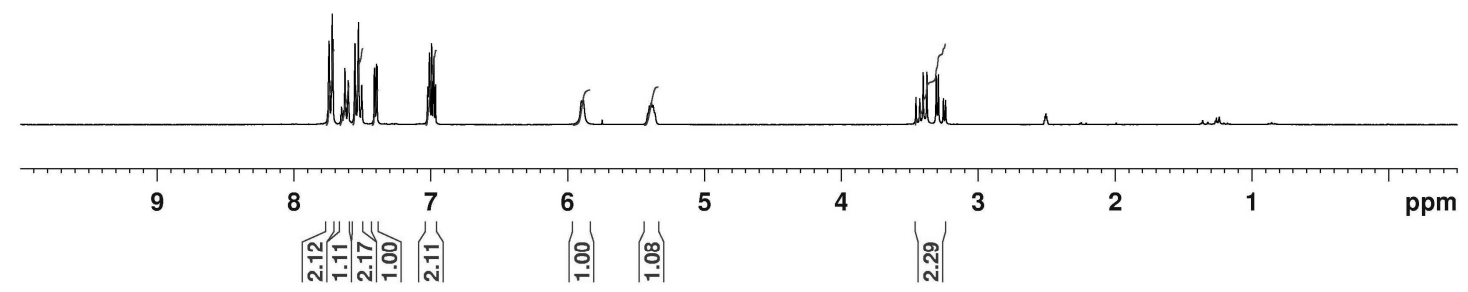

$22 \mathrm{~g}$ carbon spectrum $(75 \mathrm{MHz})$ in DMSO-d6
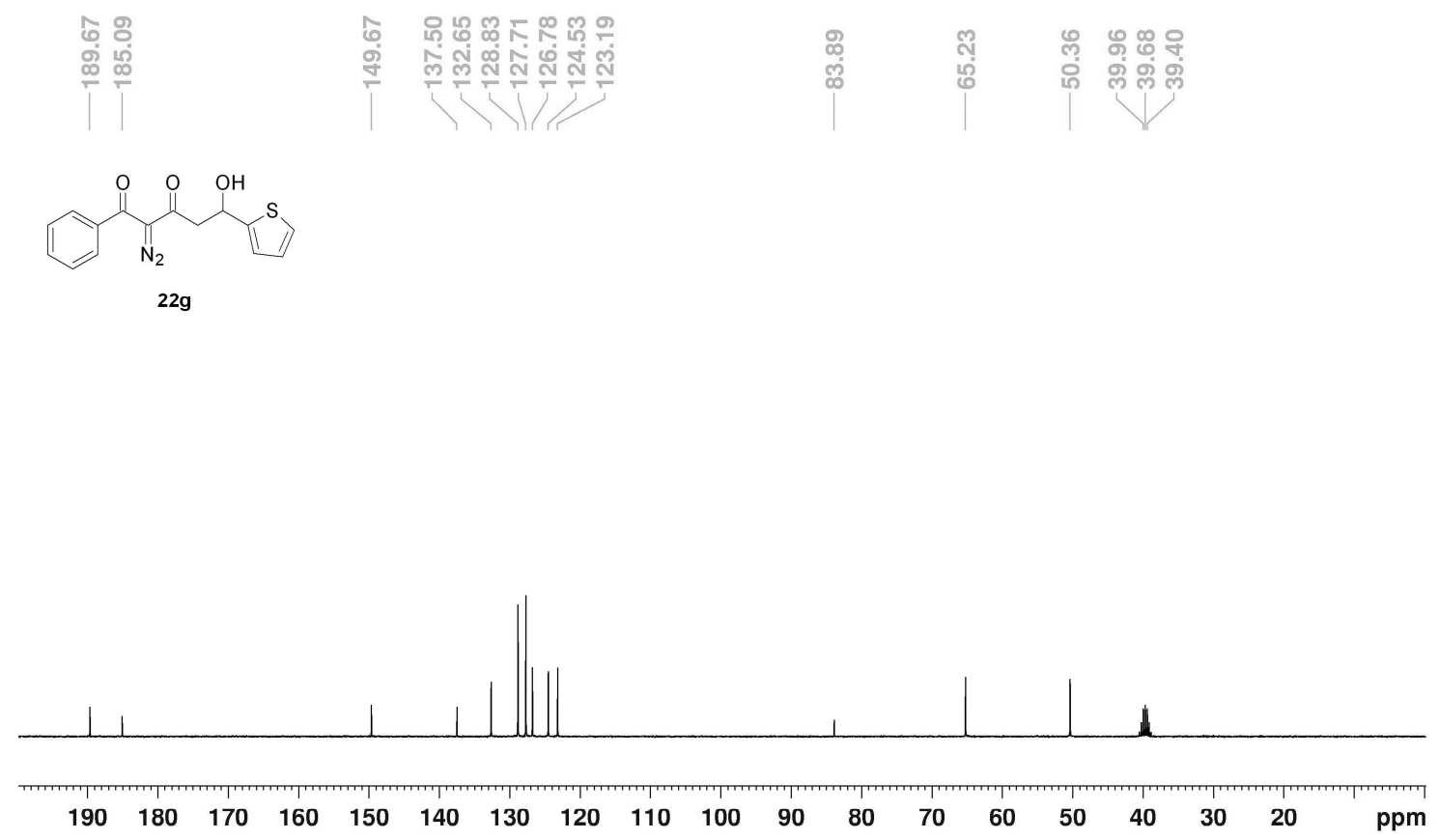
$22 \mathrm{~h}$ proton spectrum ( $300 \mathrm{MHz})$ in CDCl3

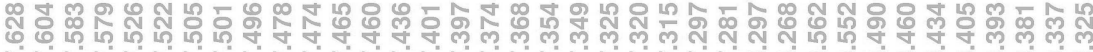

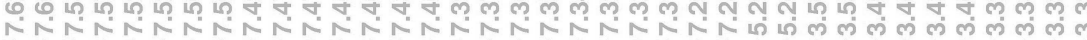

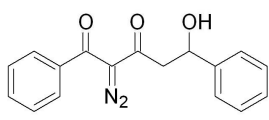

22h

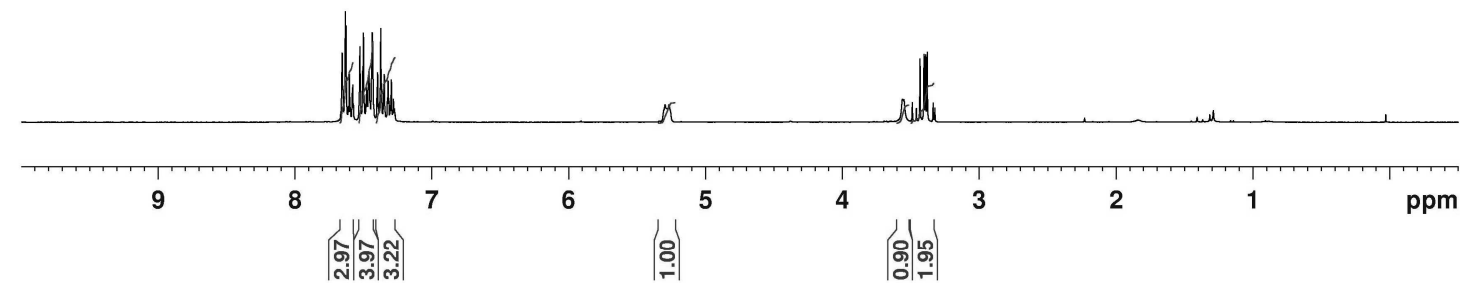

$22 \mathrm{~h}$ carbon spectrum ( $75 \mathrm{MHz})$ in $\mathrm{CDCl} 3$

๙ू ญ

ㅇำ

궁ำ

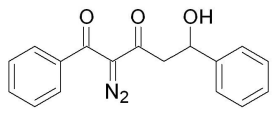

$22 \mathrm{~h}$

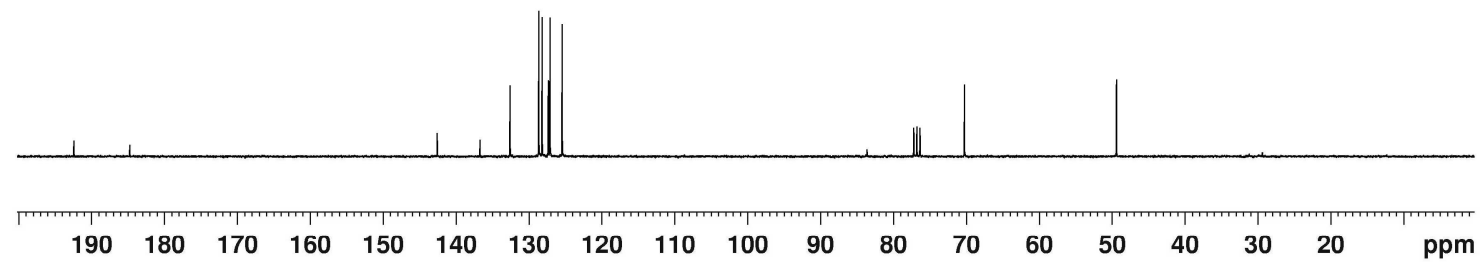




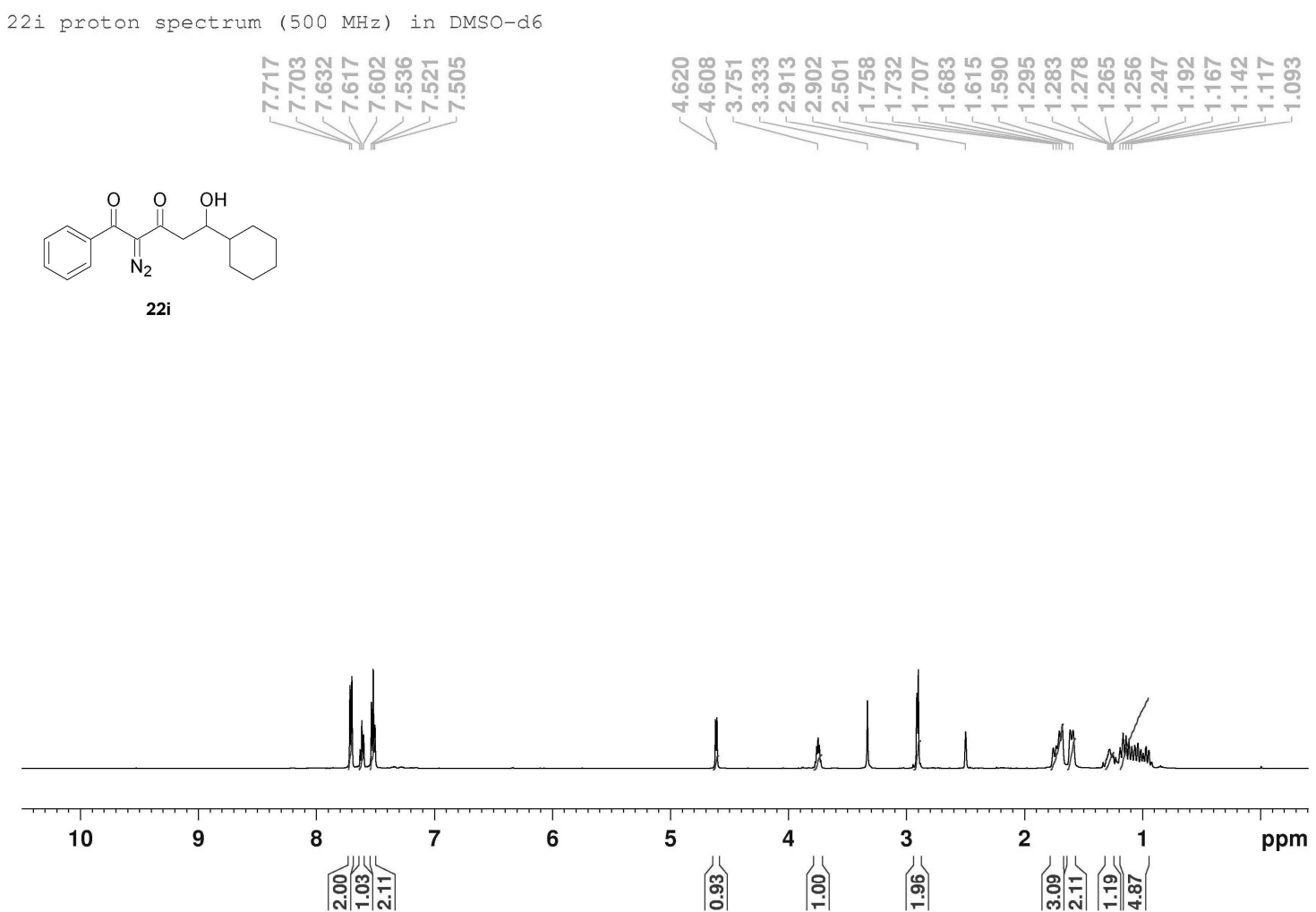

22 carbon spectrum (125 $\mathrm{MHz})$ in DMSO-d6

耐

$22 \mathbf{i}$

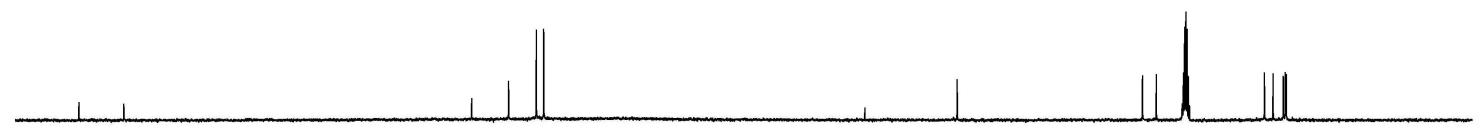

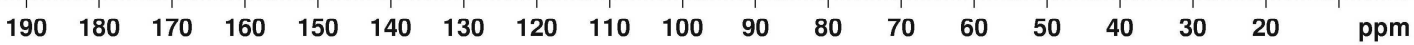


$22 j$ proton spectrum $(500 \mathrm{MHz})$ in DMSO-d6

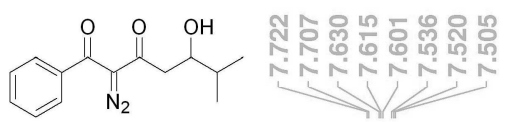

22j

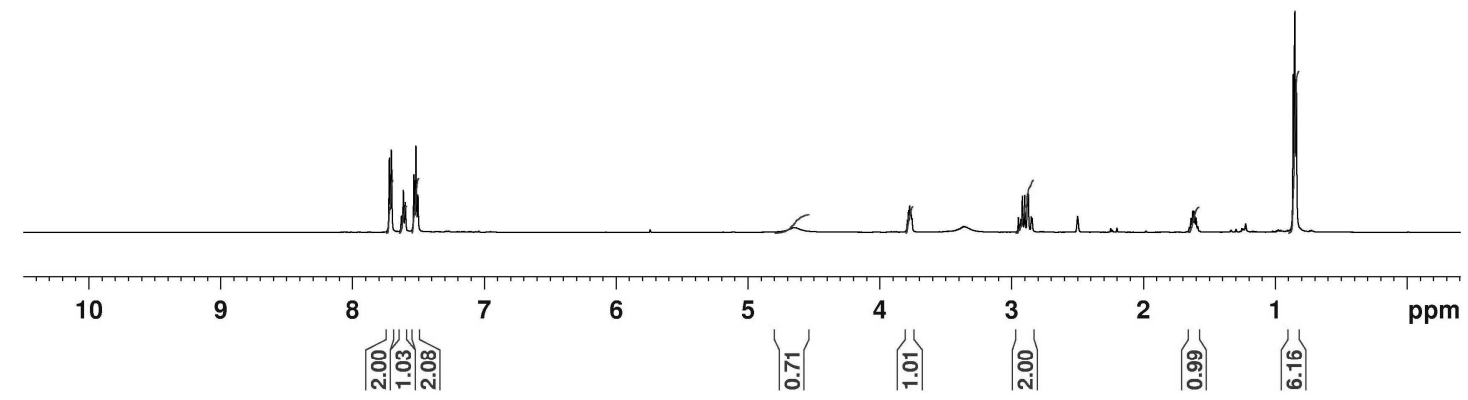

$22 j$ carbon spectrum (125 MHz) in DMSO-d6

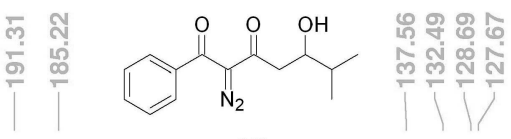

22j

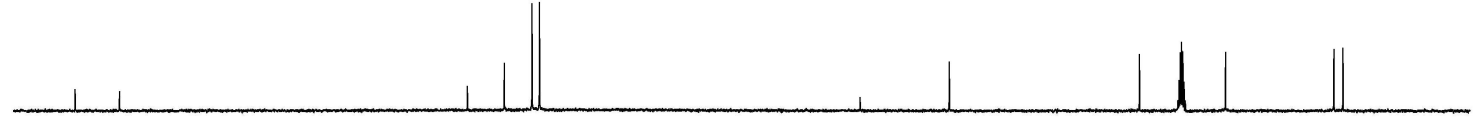

$\begin{array}{lllllllllllllllllll}190 & 180 & 170 & 160 & 150 & 140 & 130 & 120 & 110 & 100 & 90 & 80 & 70 & 60 & 50 & 40 & 30 & 20 & \mathrm{ppm}\end{array}$ 
23 proton spectrum $(500 \mathrm{MHz})$ in DMSO-d6

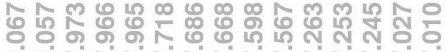

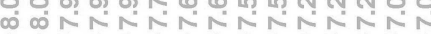

$11{ }_{\mathrm{N}_{2}}^{O}$

23

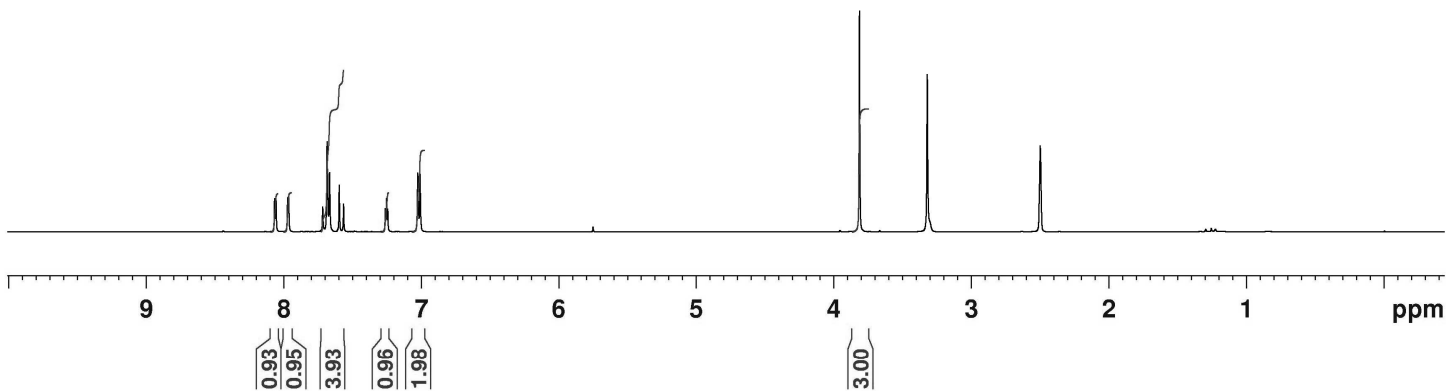

23 carbon spectrum (125 MHz) in DMSO-d6

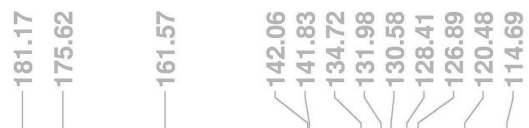
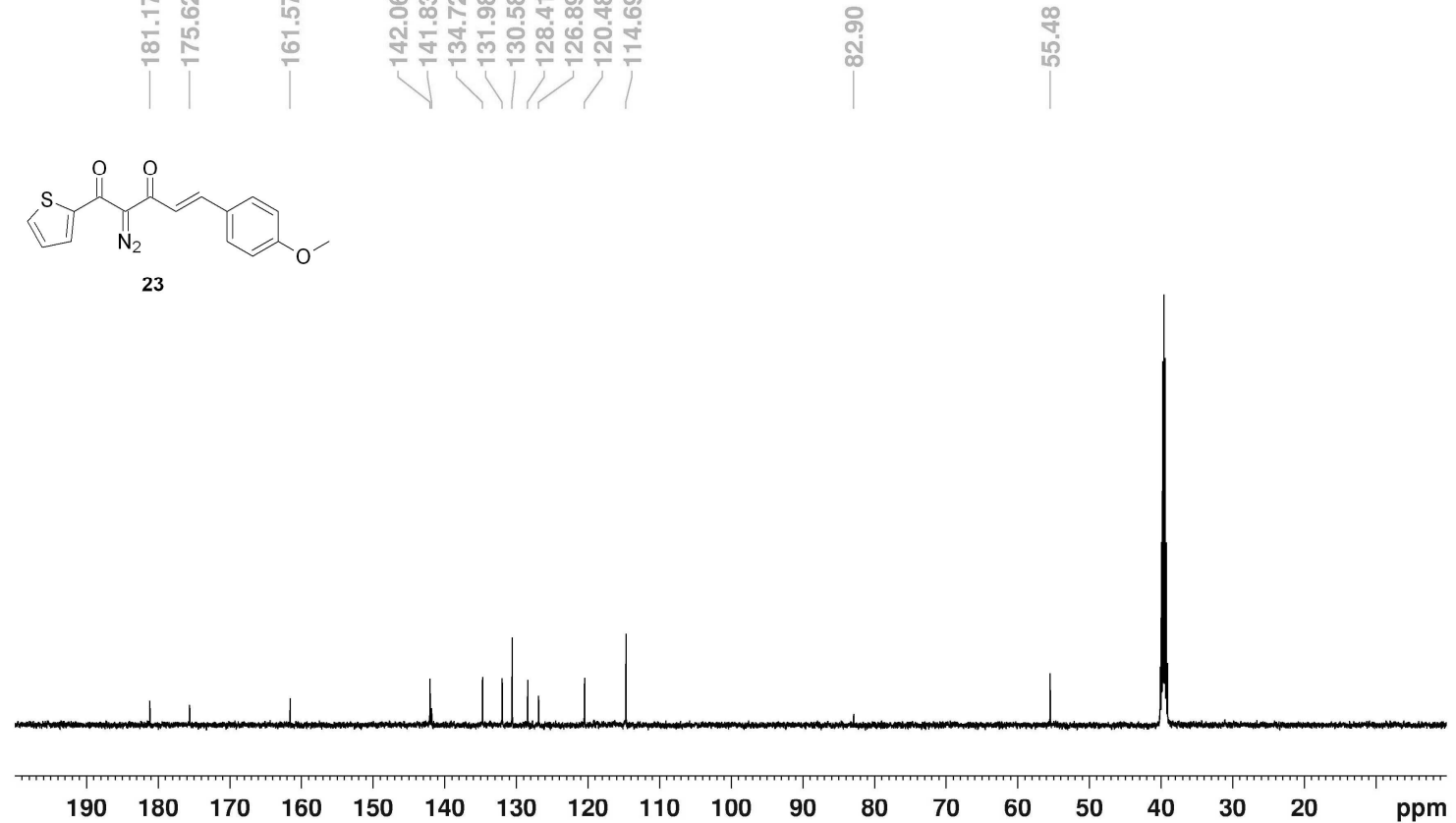
$24 \mathrm{~b}$ proton spectrum (500 MHz) in DMSO-d6

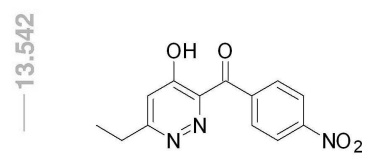

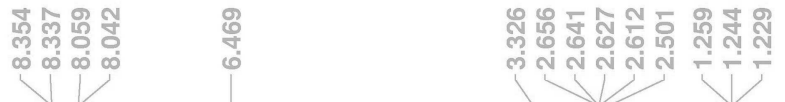

24b

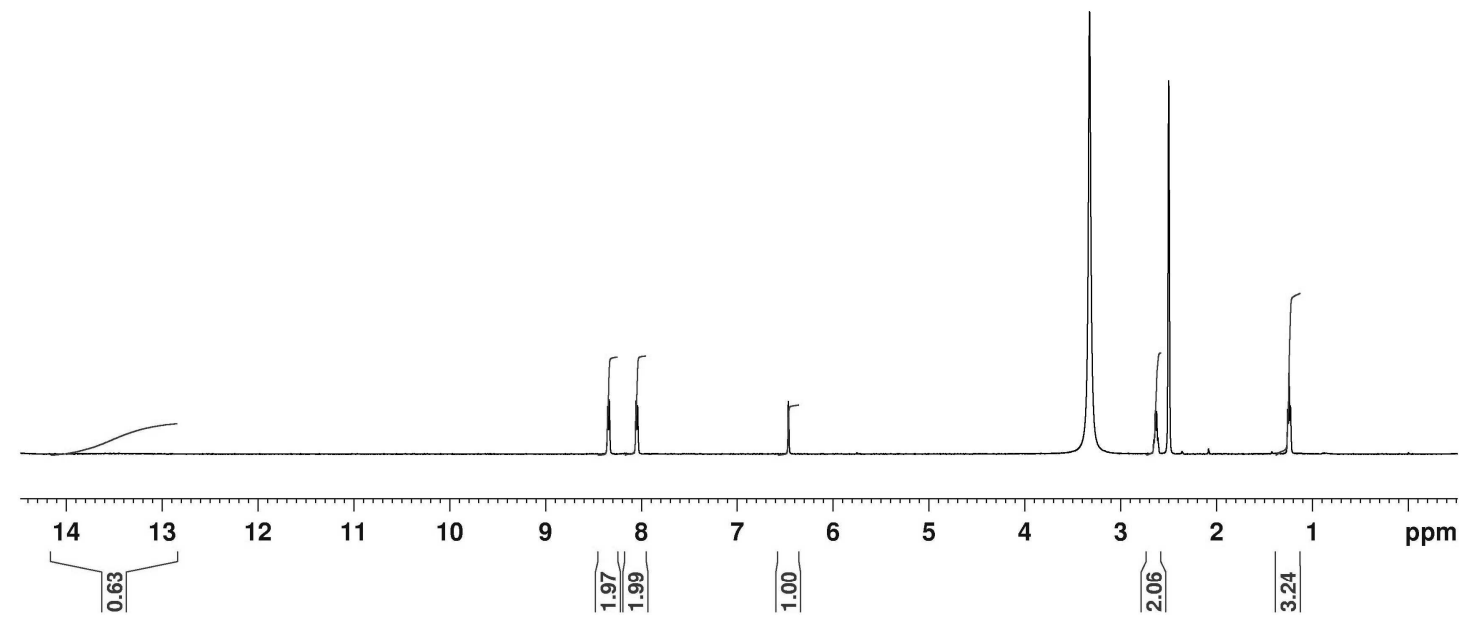

$24 \mathrm{~b}$ carbon spectrum (125 MHz) in DMSO-d6

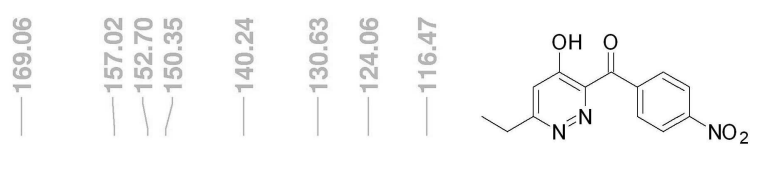

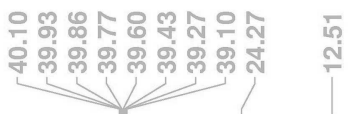

$24 b$

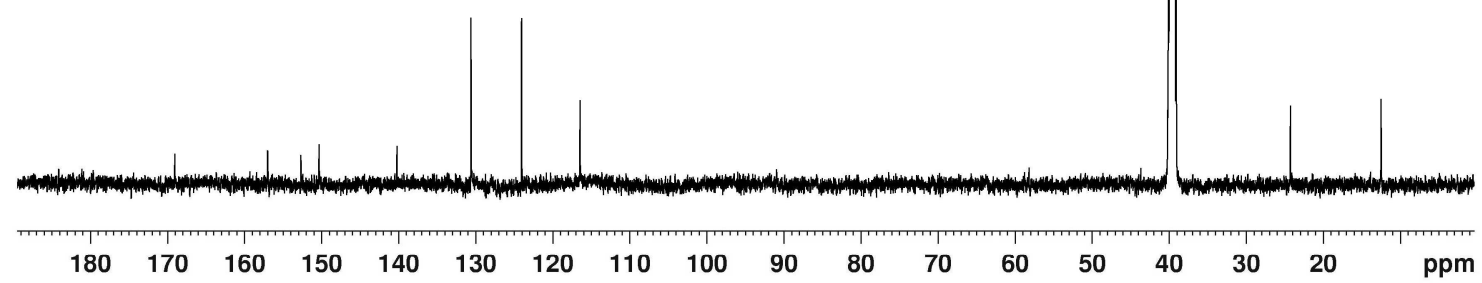


$24 \mathrm{C}$ proton spectrum $(600 \mathrm{MHz})$ in DMSO-d6

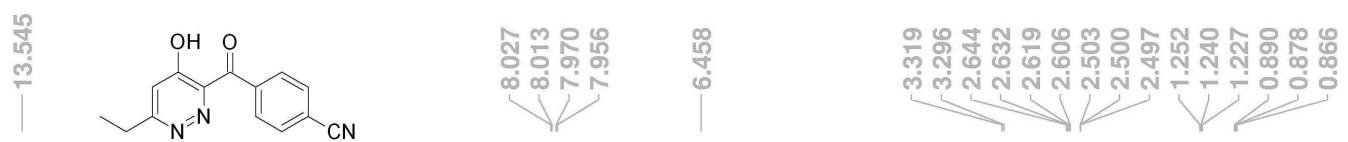

24c

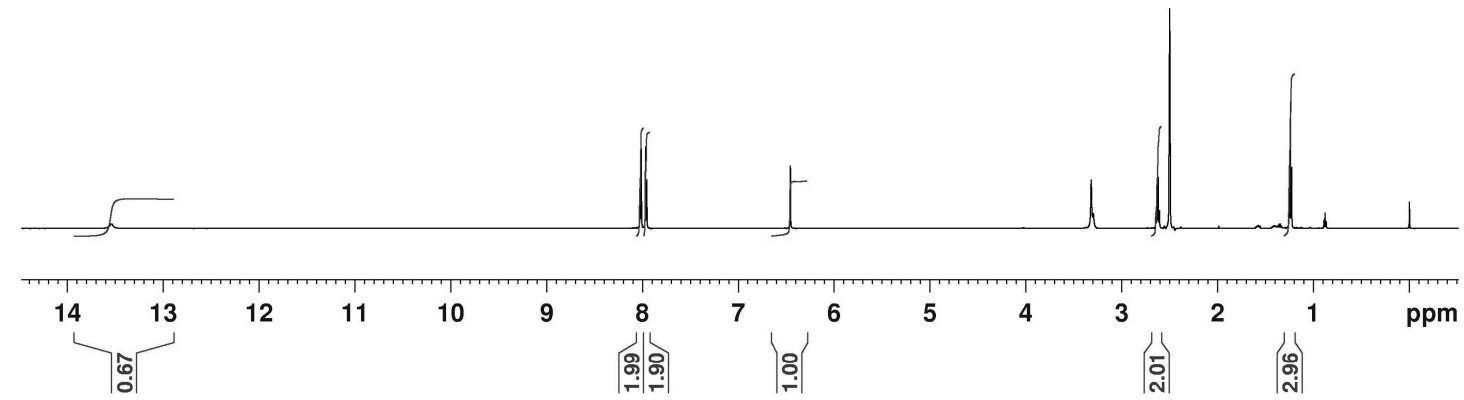

24c carbon spectrum (150 MHz) in DMSO-d6

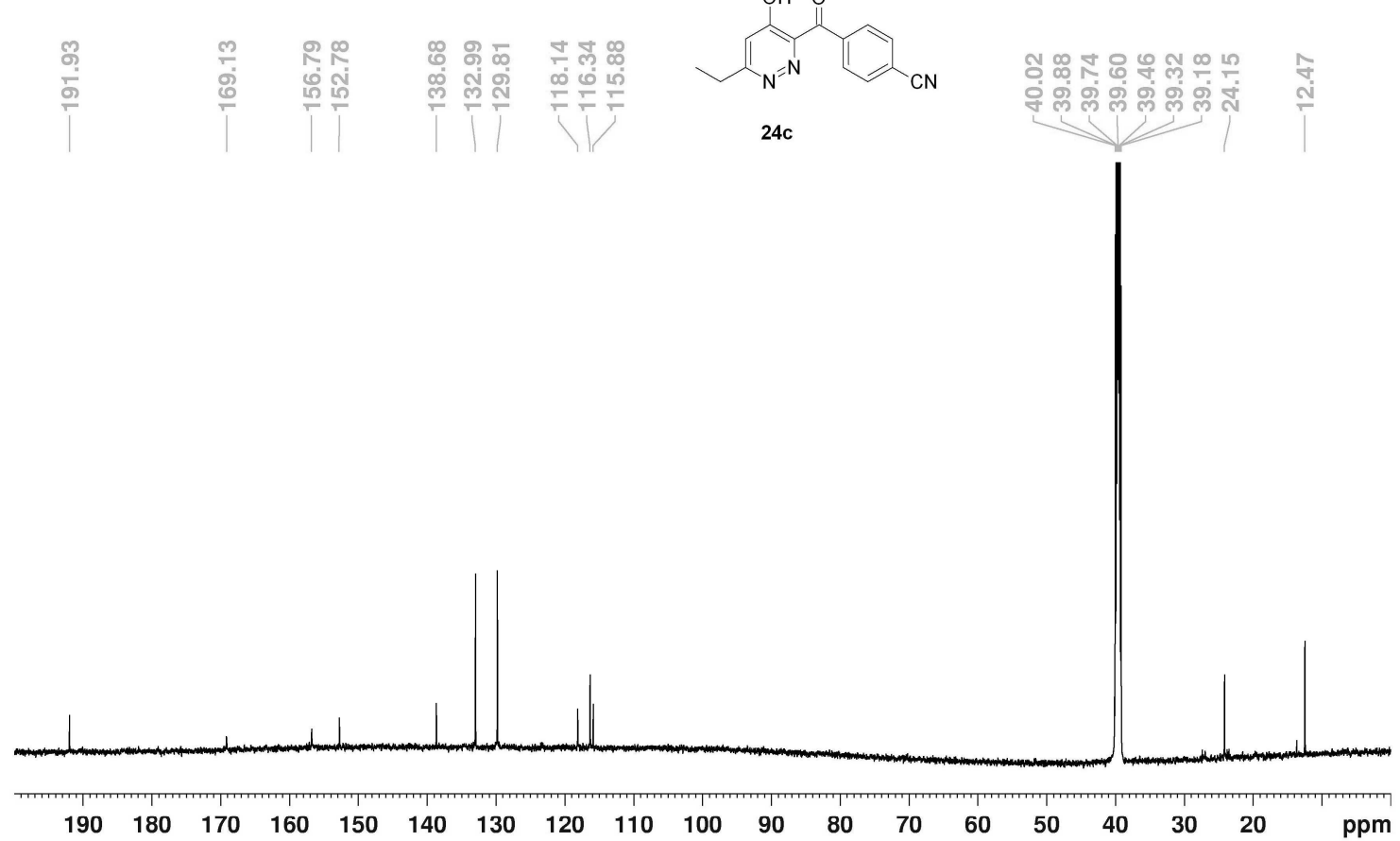


$24 \mathrm{~d}$ proton spectrum $(600 \mathrm{MHz})$ in DMSO-d6
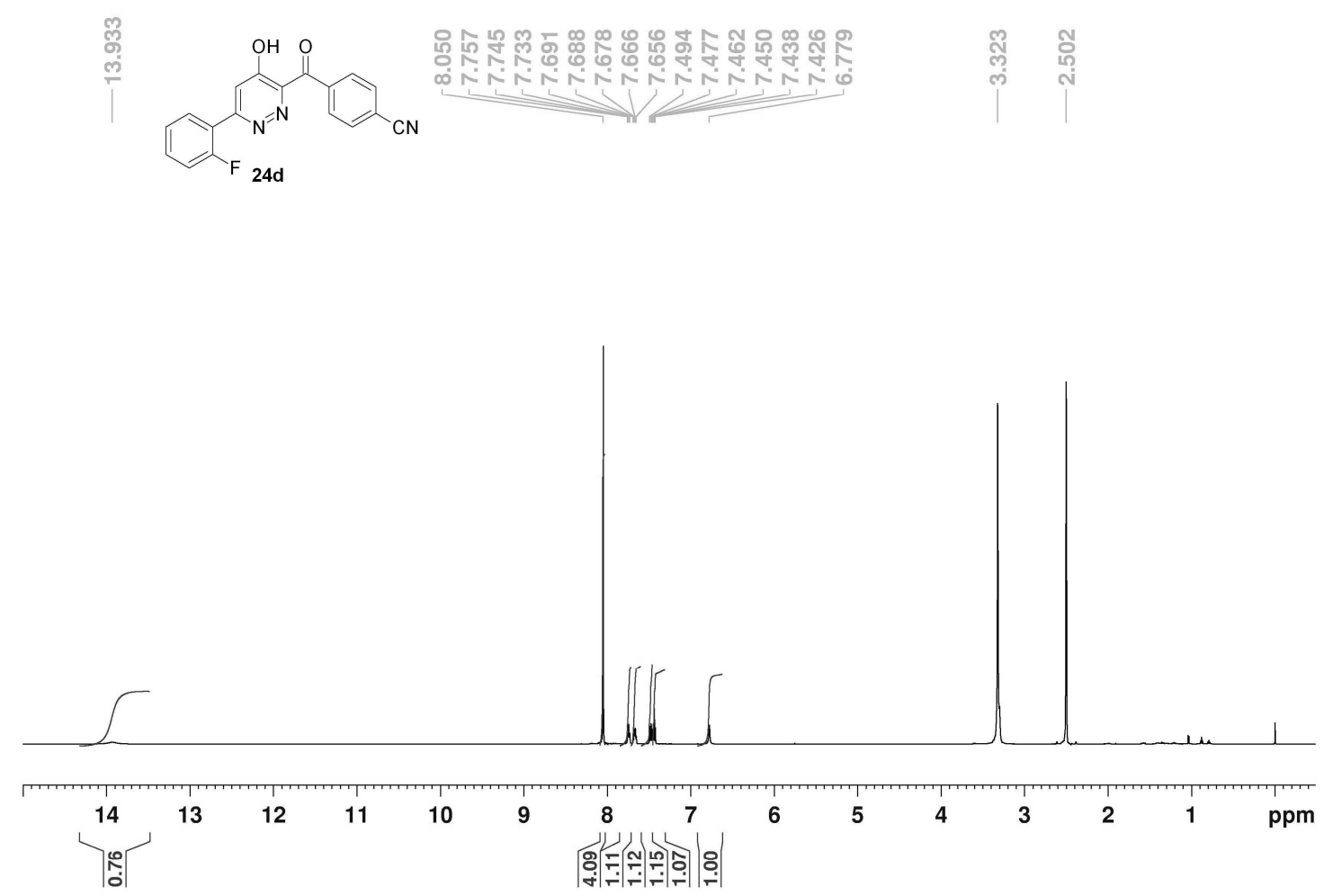

24d carbon spectrum (150 MHz) in DMSO-d6

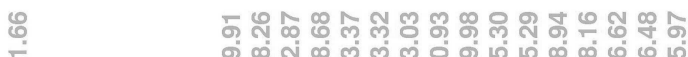

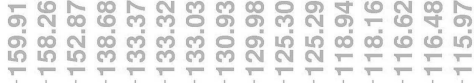

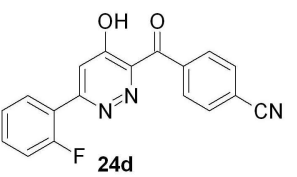

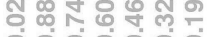

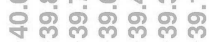

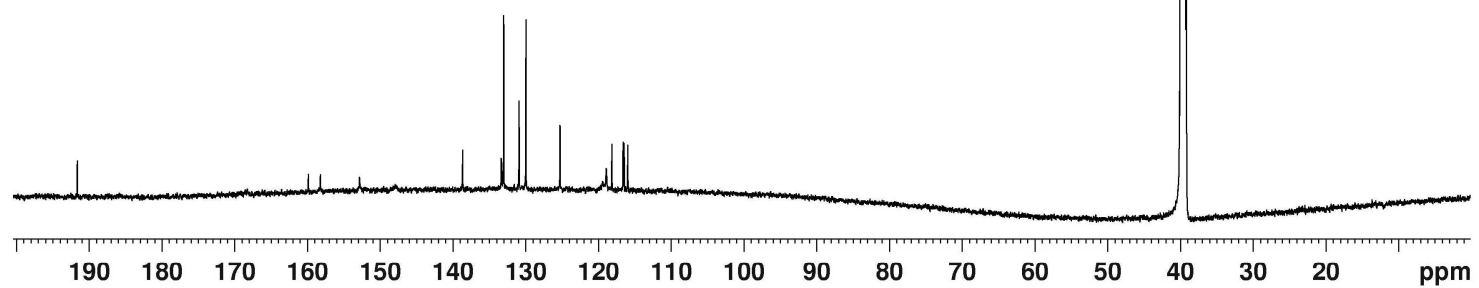


24 e proton spectrum (600 MHz) in DMSO-d6

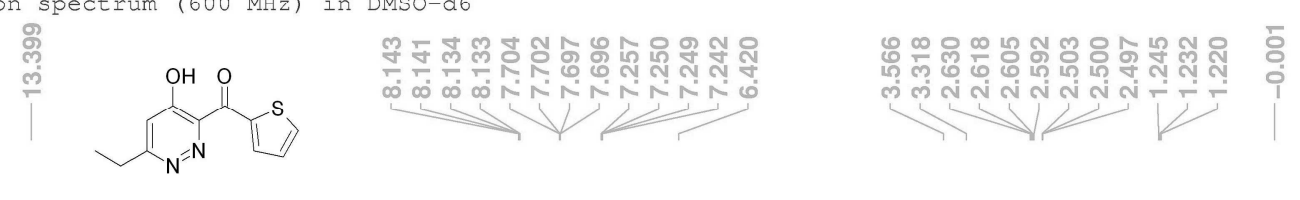

$24 \mathrm{e}$
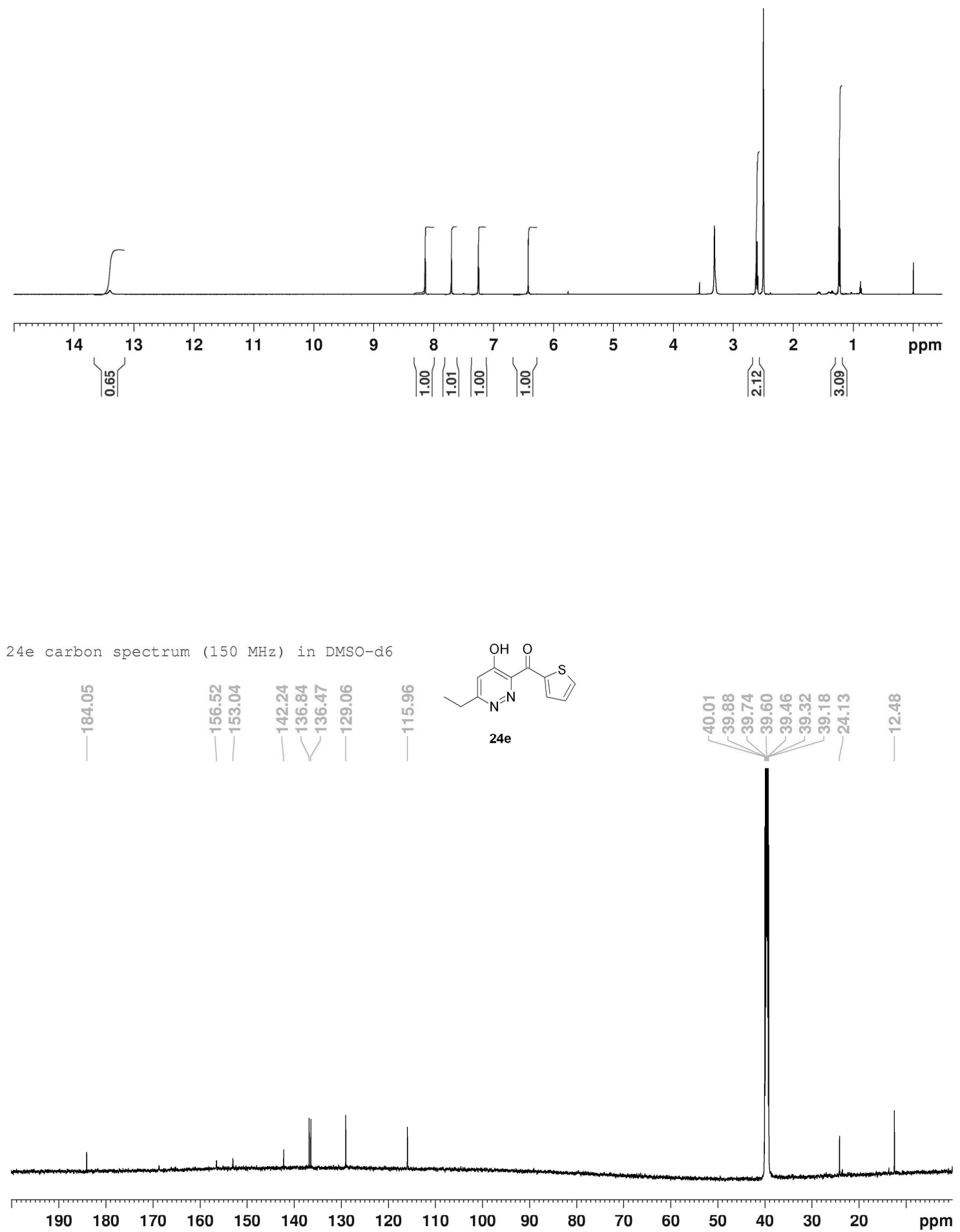
$21 \mathrm{f}$ proton spectrum (600 MHz) in DMSO-d6

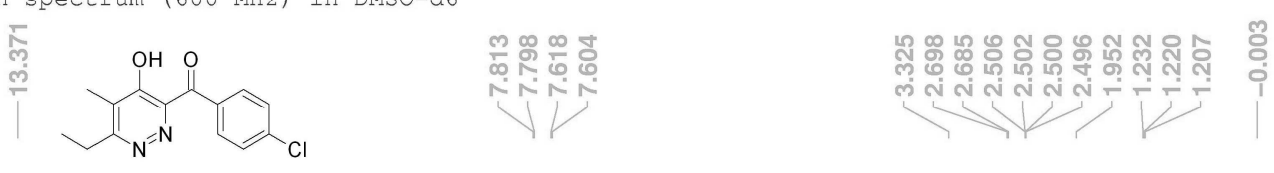

$24 f$

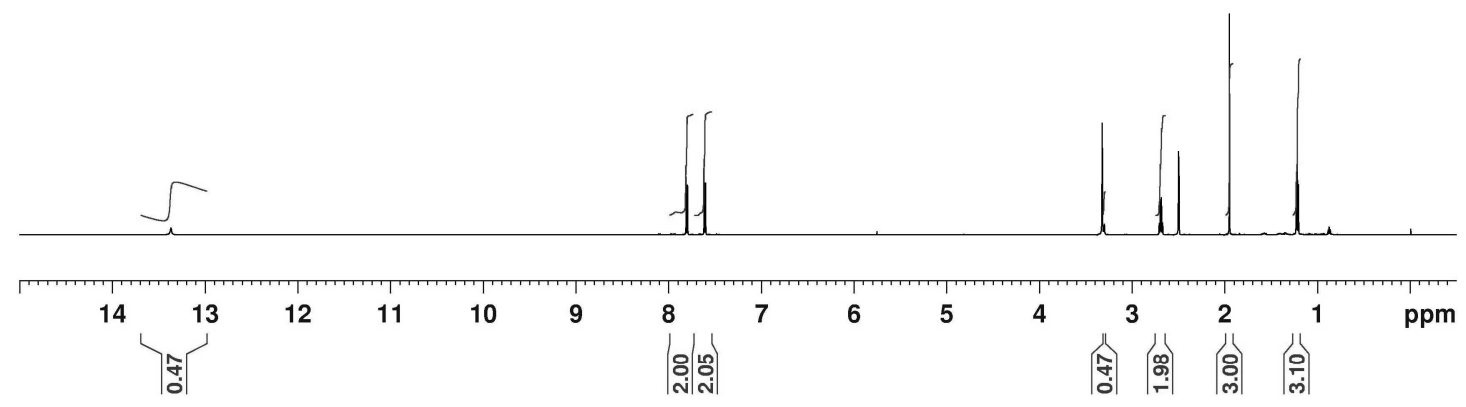

$24 \mathrm{f}$ carbon spectrum (150 MHz) in DMSO-d6

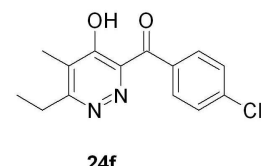

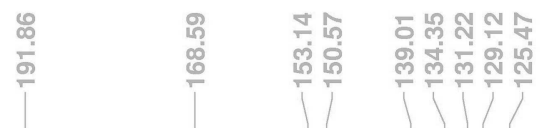

$24 f$

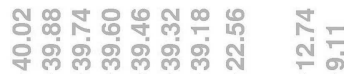

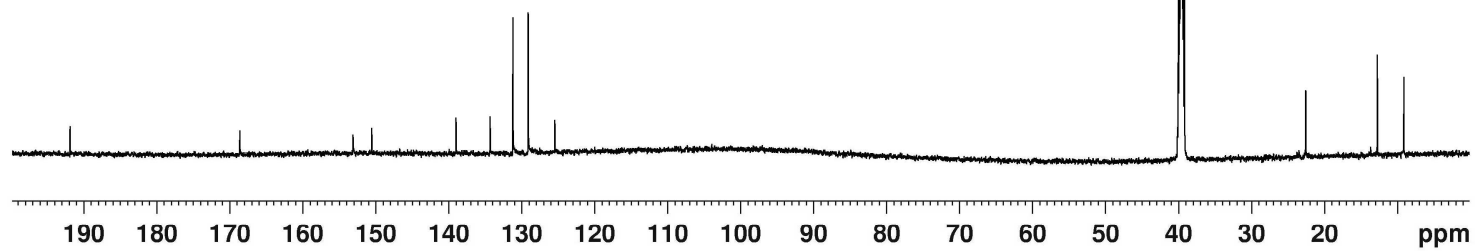


$24 \mathrm{~g}$ proton spectrum (600 MHz) in DMSO-d6

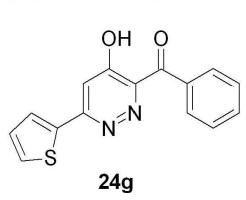

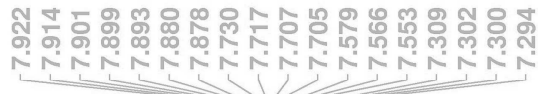

둥요웜

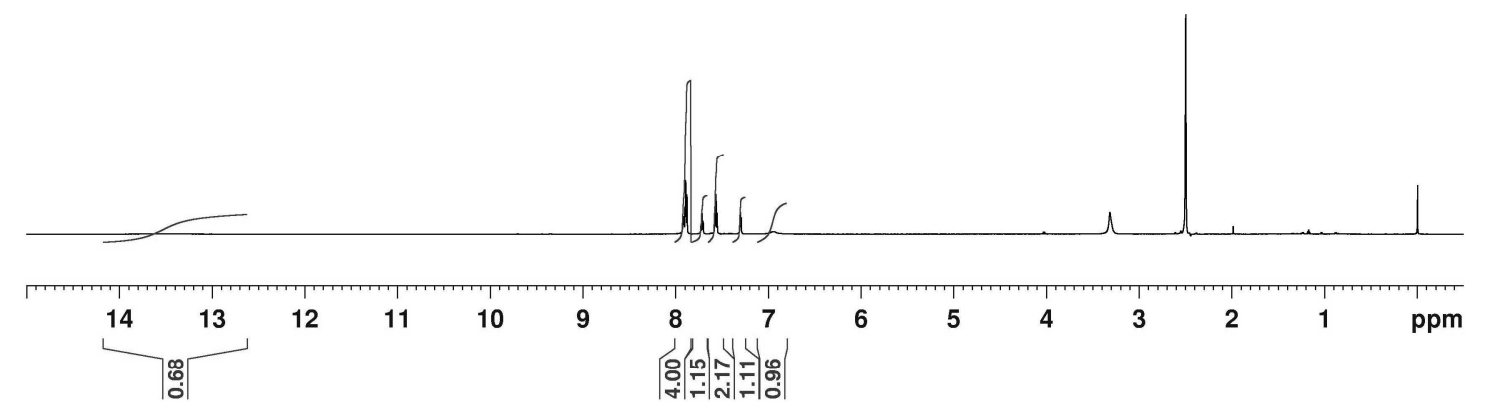

$24 \mathrm{~g}$ carbon spectrum (150 MHz) in DMSO-d6

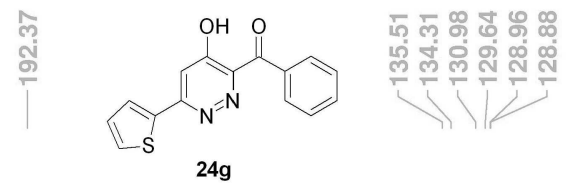

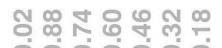

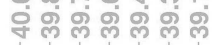
. 


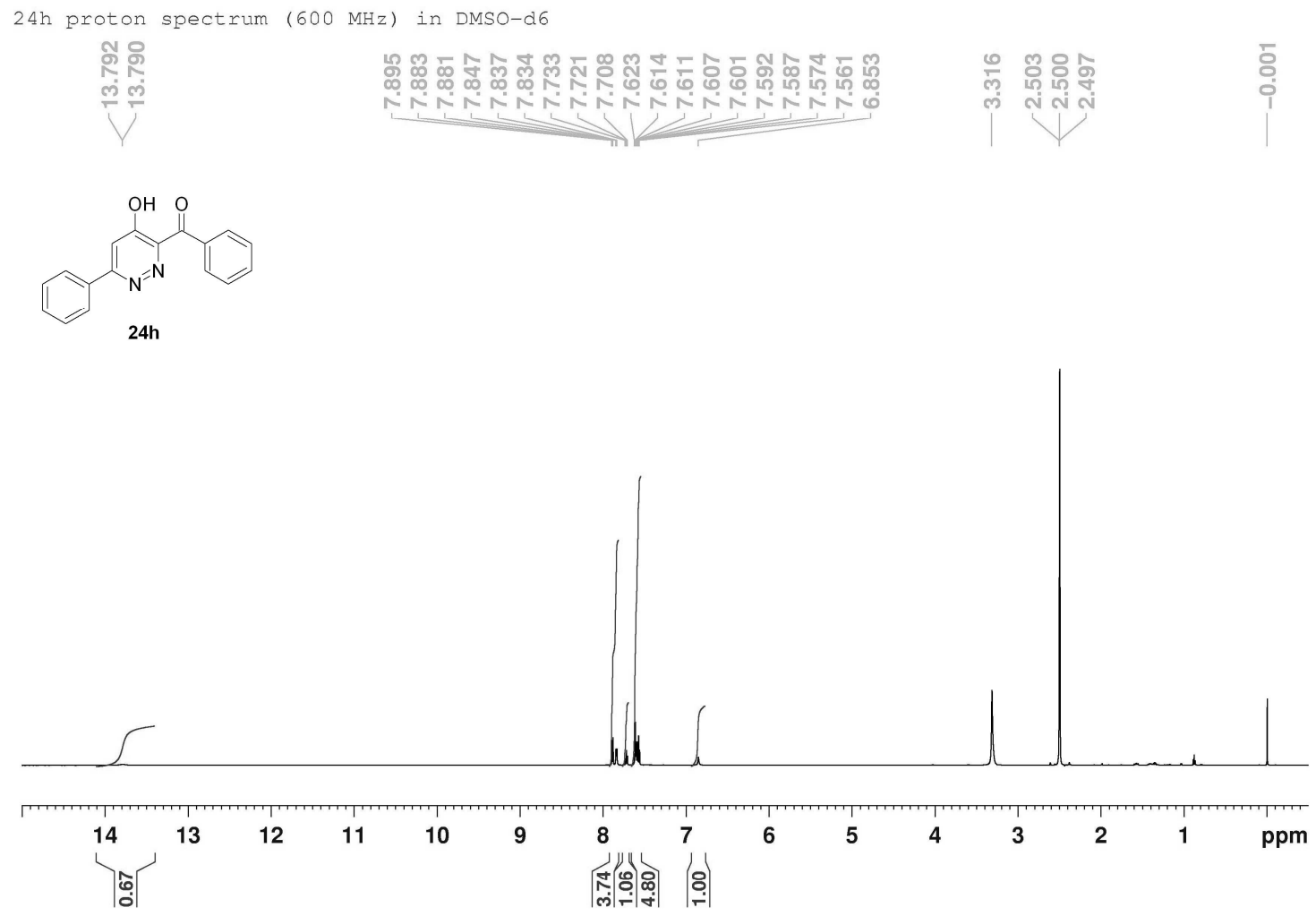

$24 \mathrm{~h}$ carbon spectrum (150 MHz) in DMSO-d6
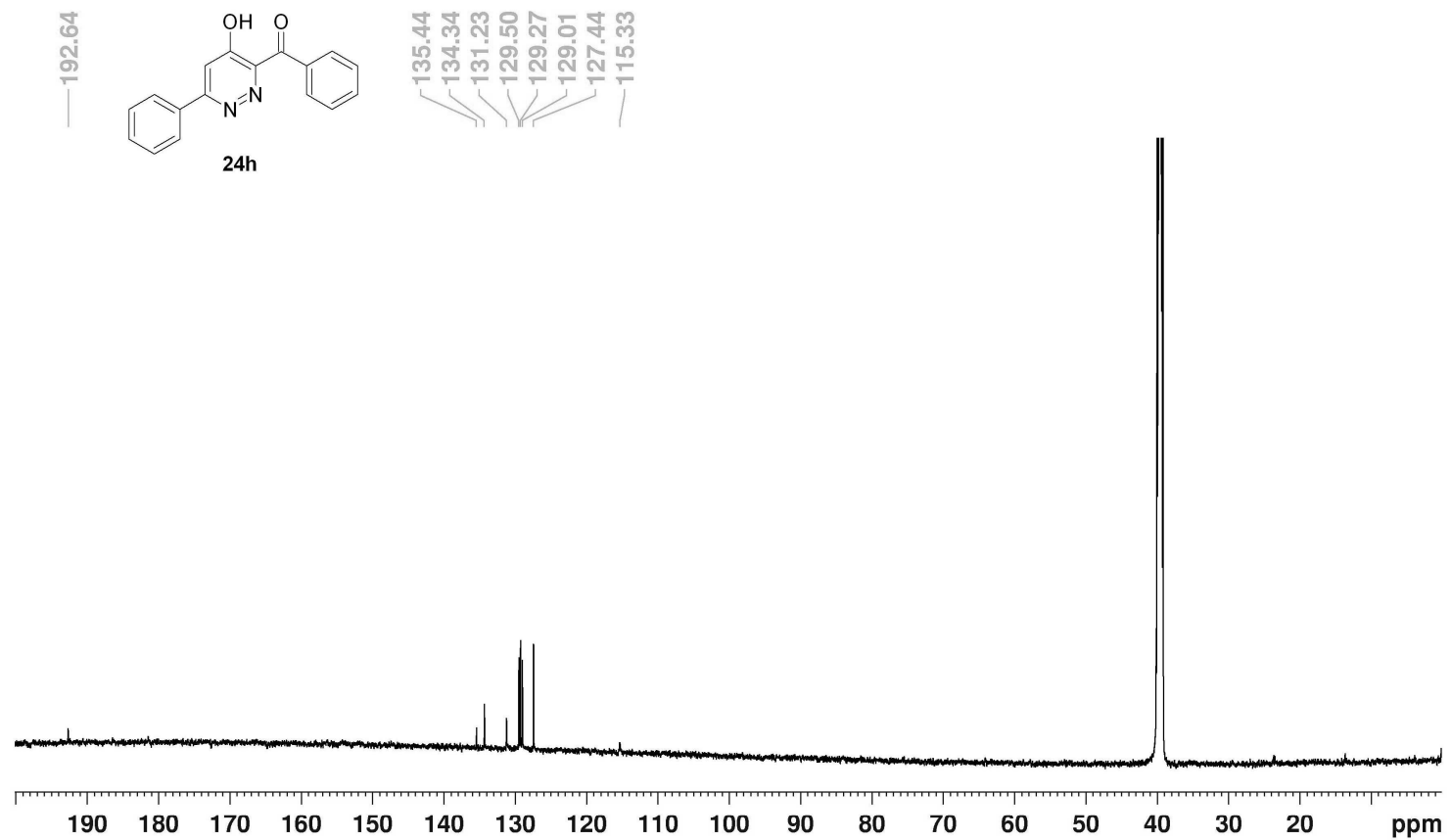
$24 i$ proton spectrum ( $600 \mathrm{MHz})$ in DMSO-d6

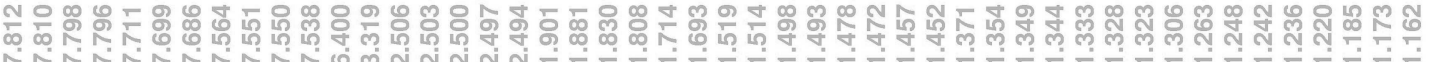
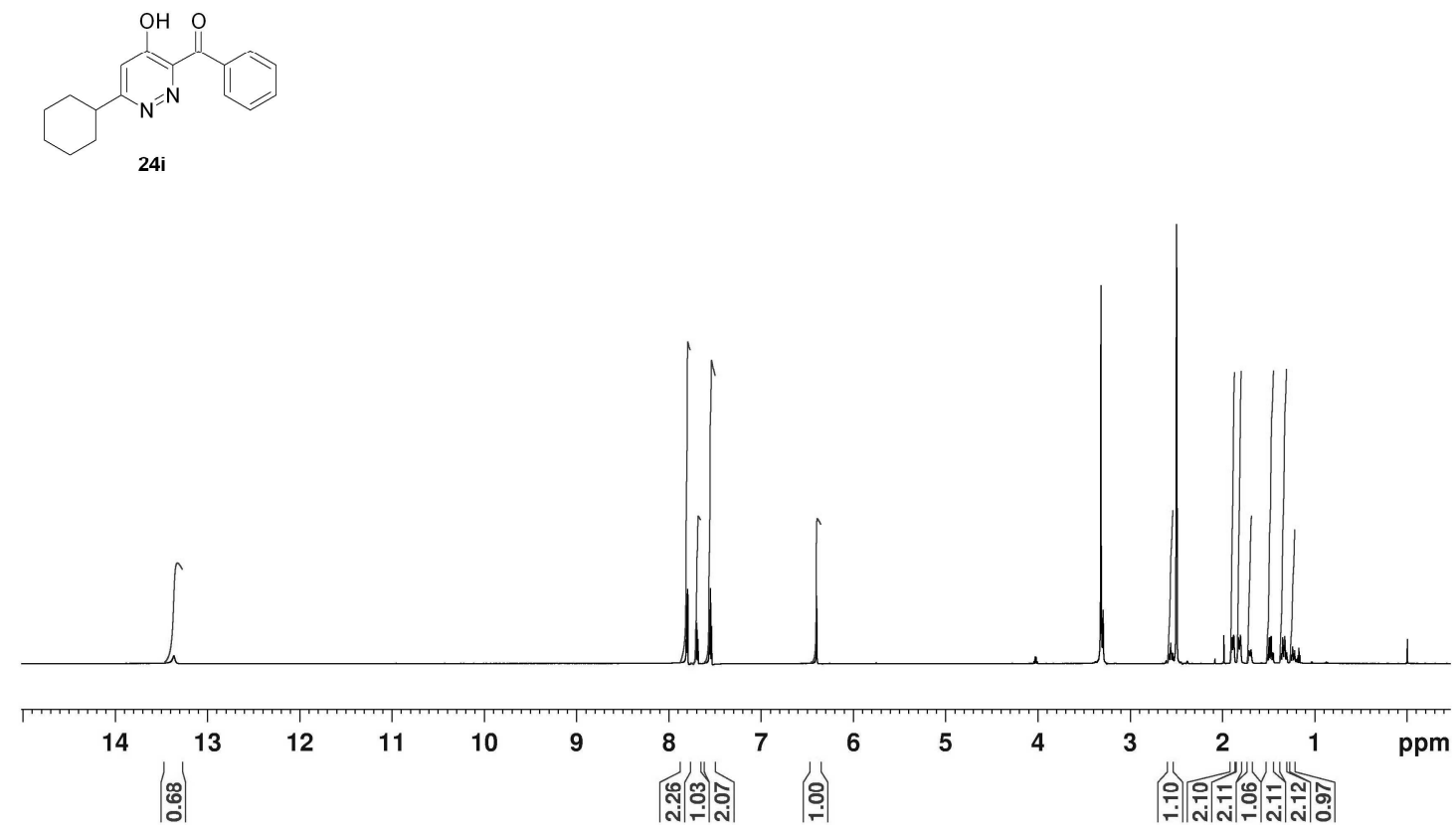

$24 i$ carbon spectrum (150 MHz) in DMSO-d6
N
กั
意億

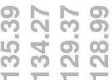
্ํㅁ

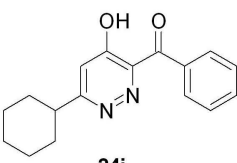

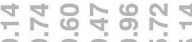

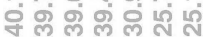
24i

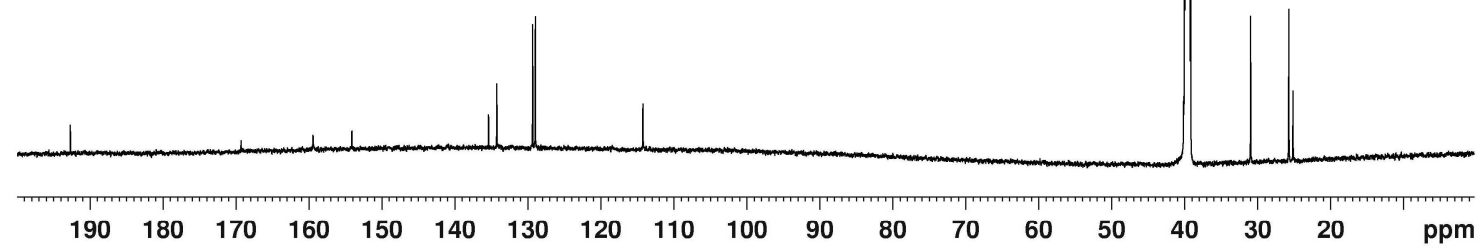


$24 j$ proton spectrum (600 MHz) in DMSO-d6
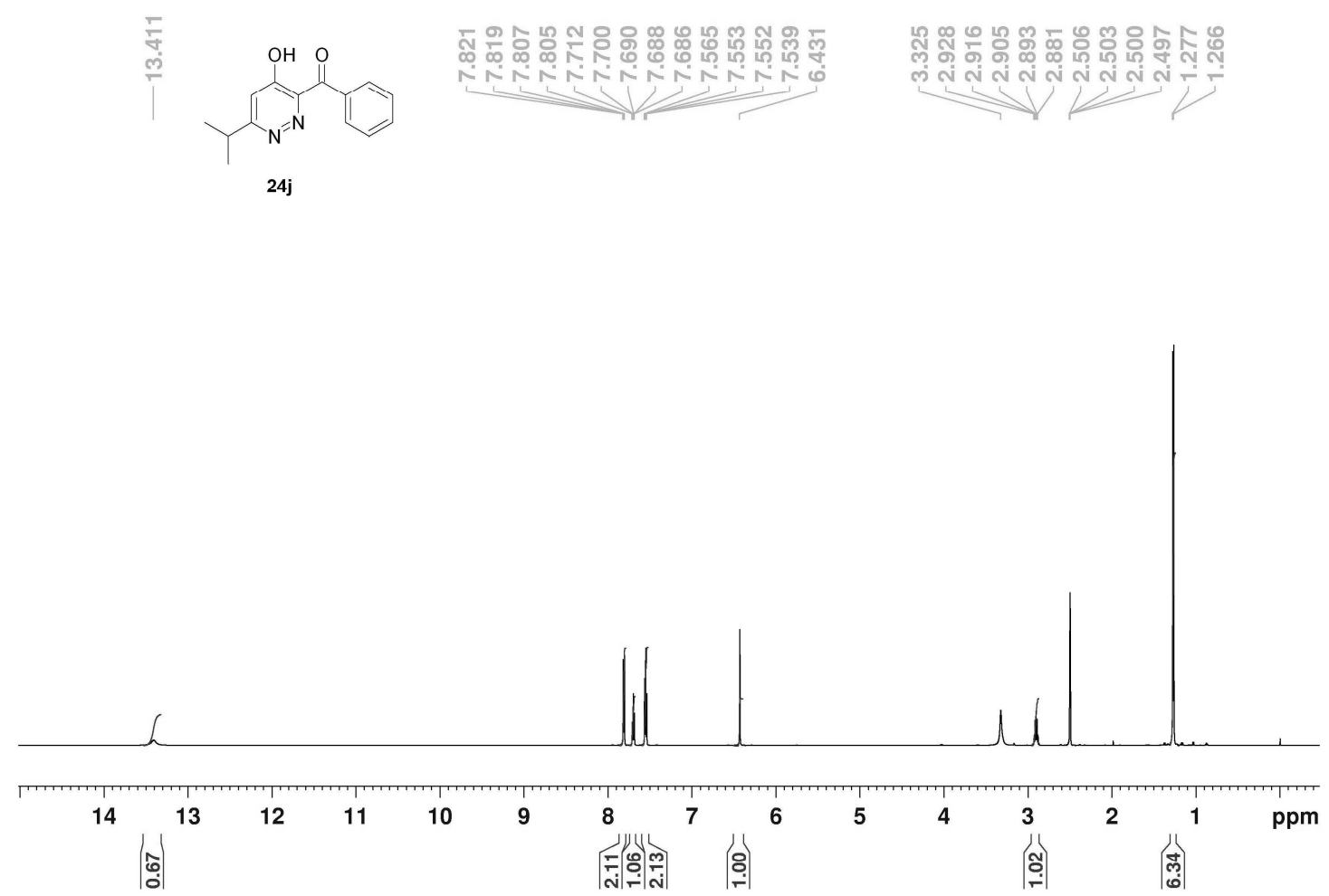

$24 j$ carbon spectrum (150 MHz) in DMSO-d6

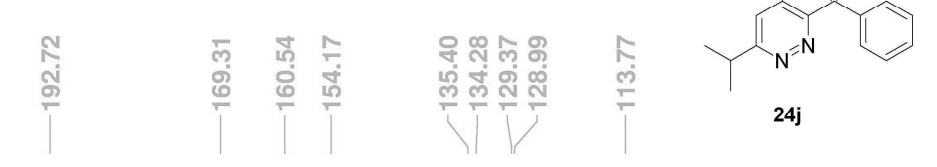

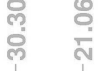

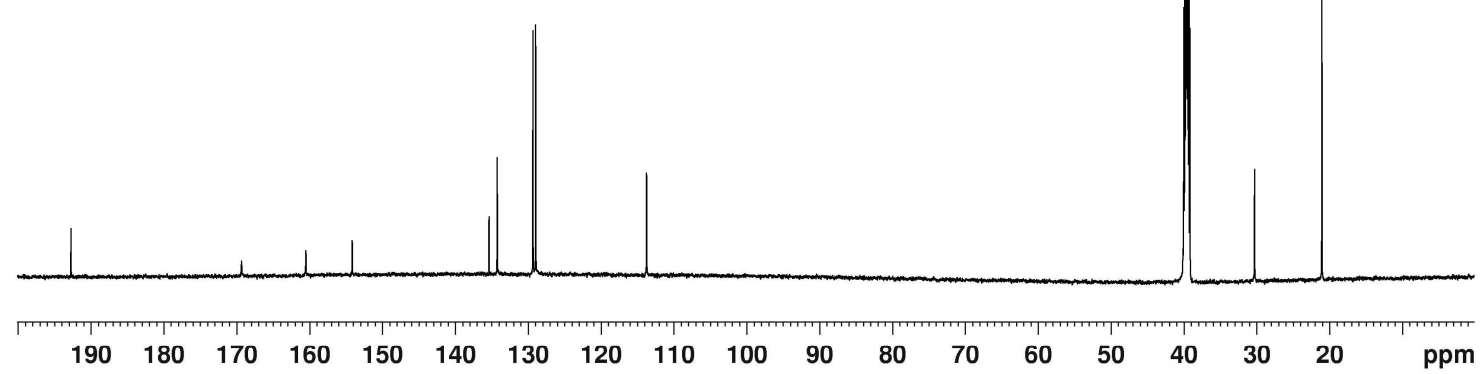


25 a proton spectrum $(600 \mathrm{MHz})$ in DMSO-d6

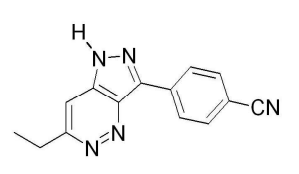

$25 a$

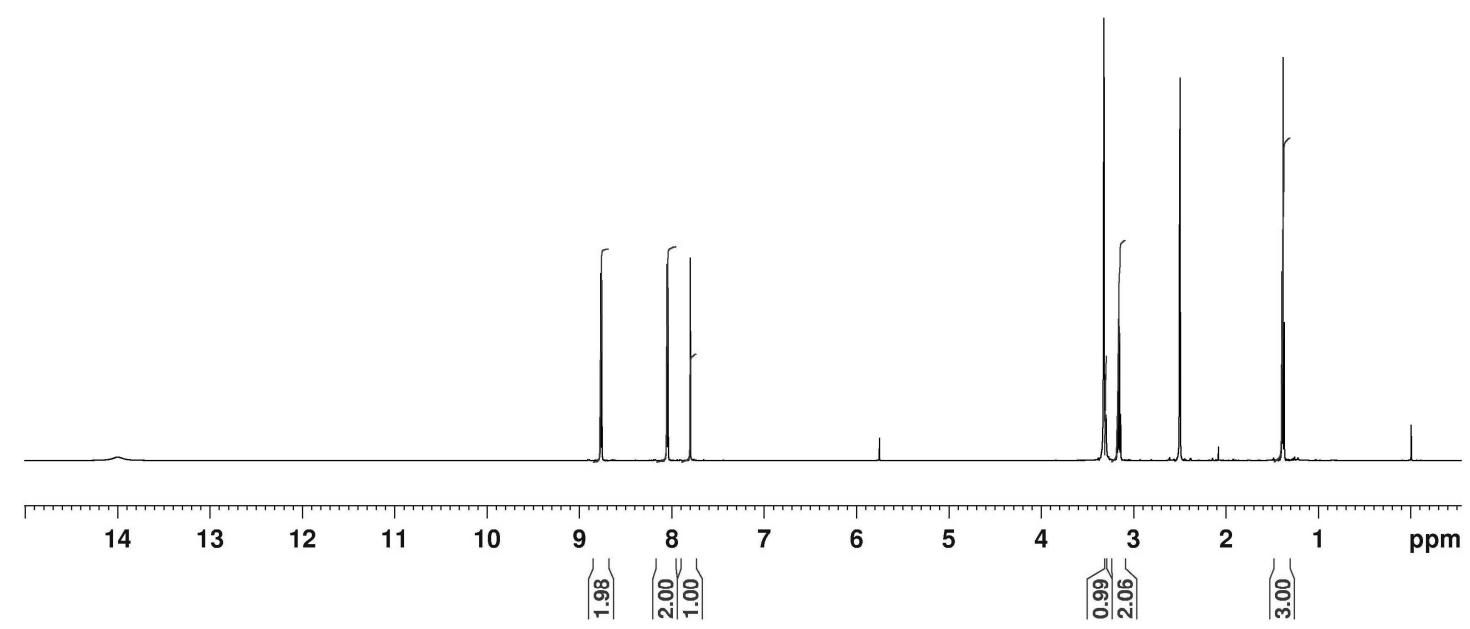

$25 a$ carbon spectrum (150 $\mathrm{MHz}$ ) in DMSO-d6

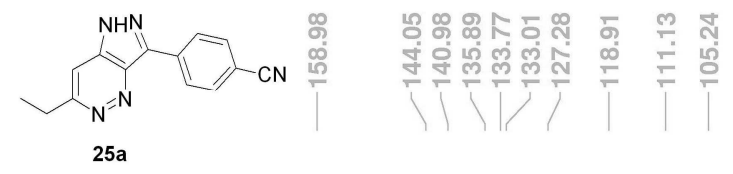

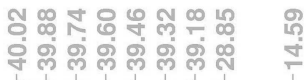

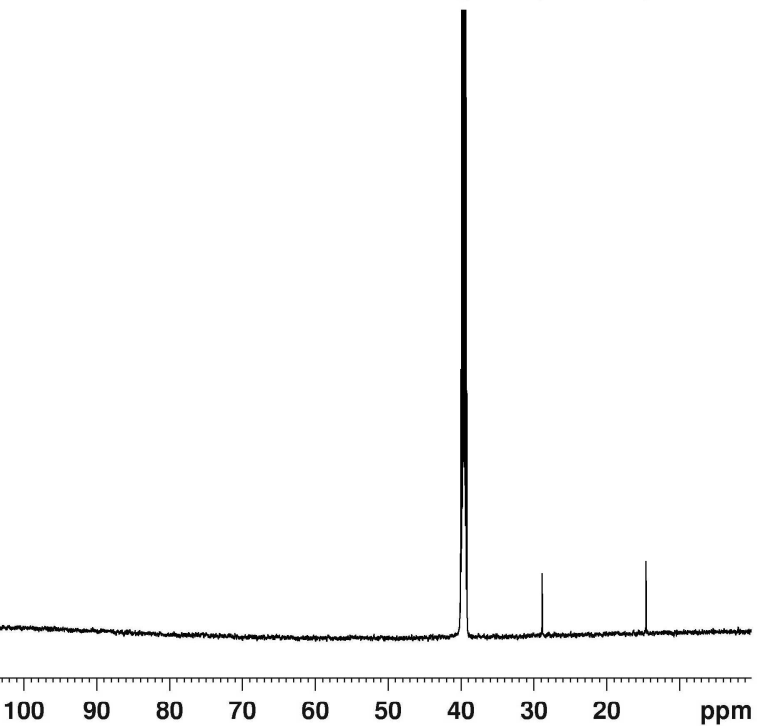


$25 \mathrm{~b}$ proton spectrum (300 $\mathrm{MHz})$ in DMSO-d6

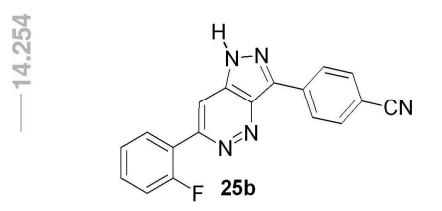

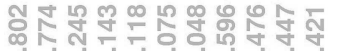

$\infty \infty \infty \infty \infty \infty)$

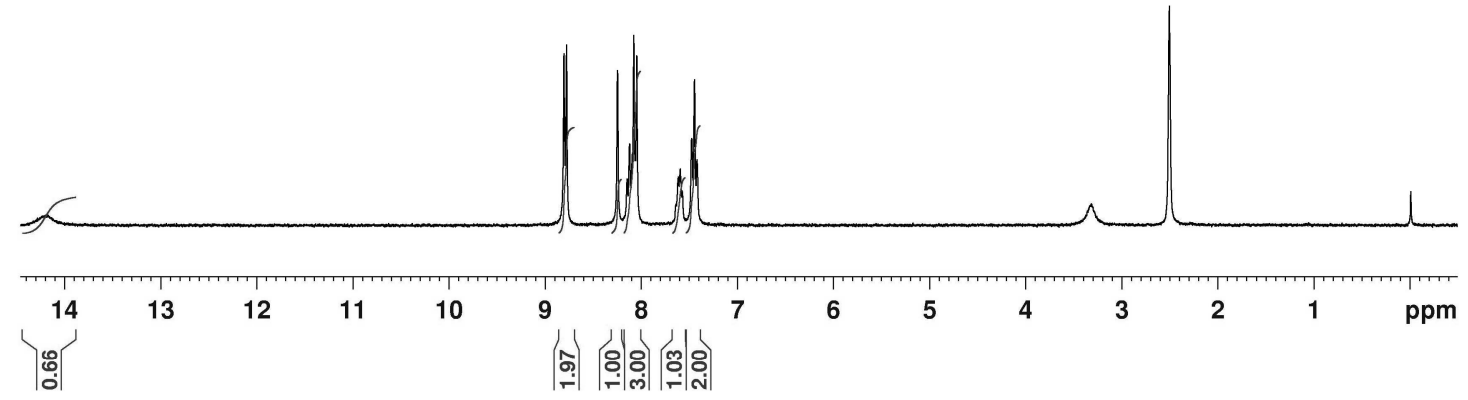

25b carbon spectrum ( $75 \mathrm{MHz})$ in DMSO-d6
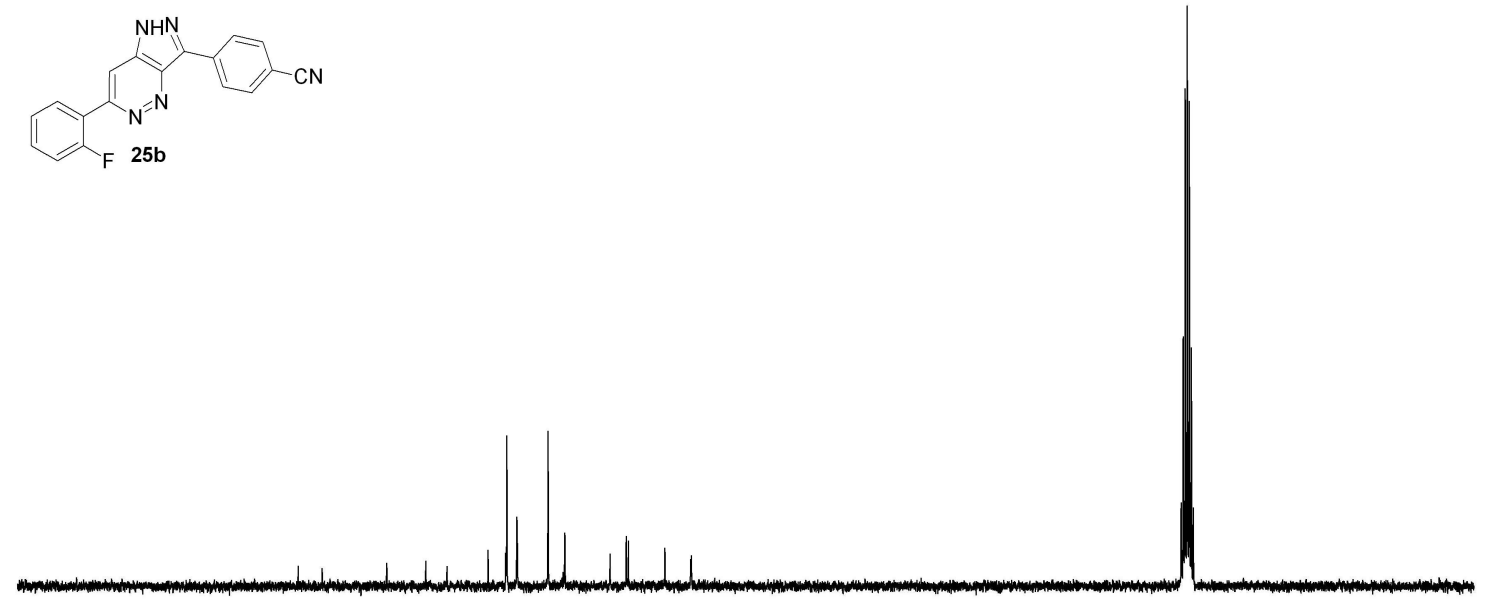

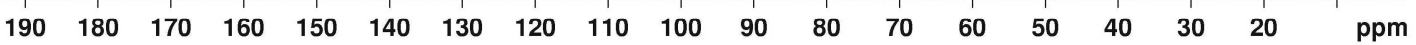


$25 \mathrm{c}$ proton spectrum (500 MHz) in DMSO-d6

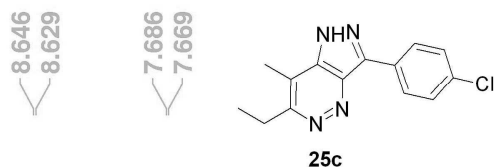

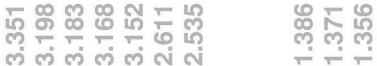

25c

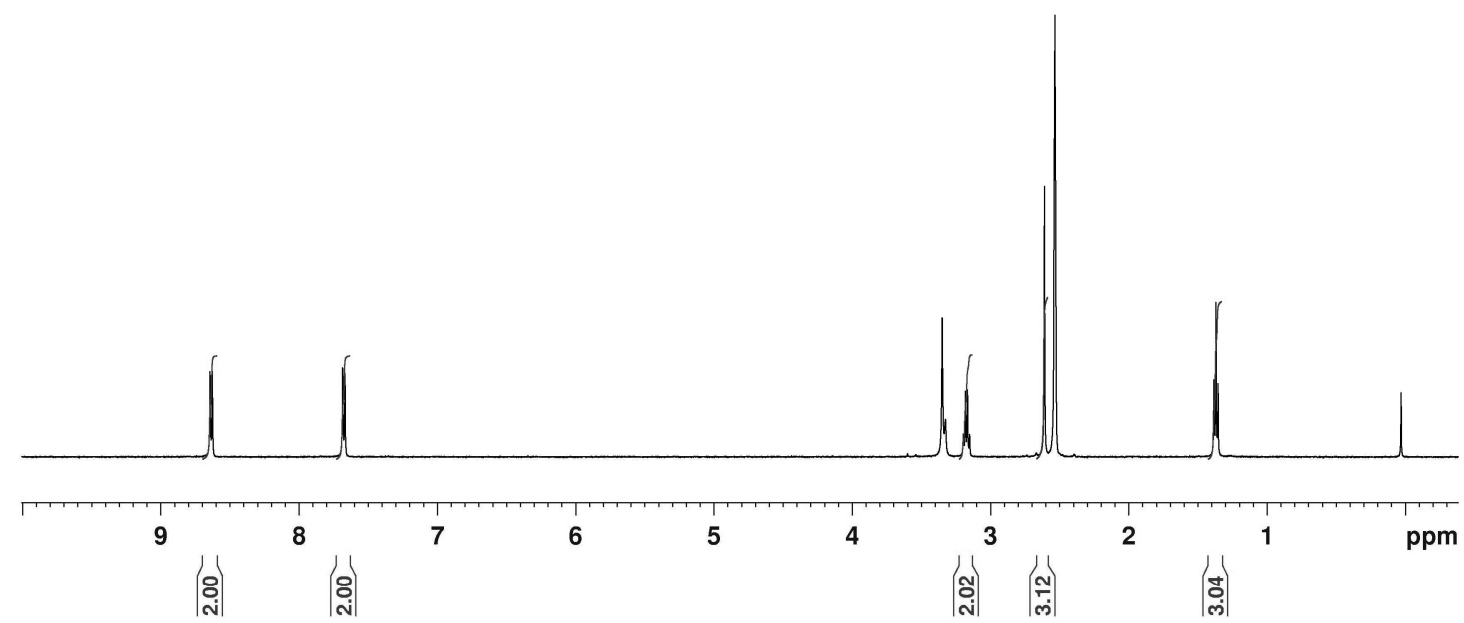

$25 \mathrm{c}$ carbon spectrum ( $125 \mathrm{MHz}$ ) in DMSO-d6

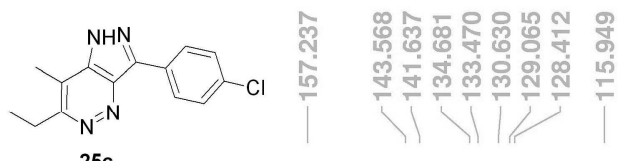

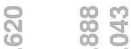

แึ่ लำ

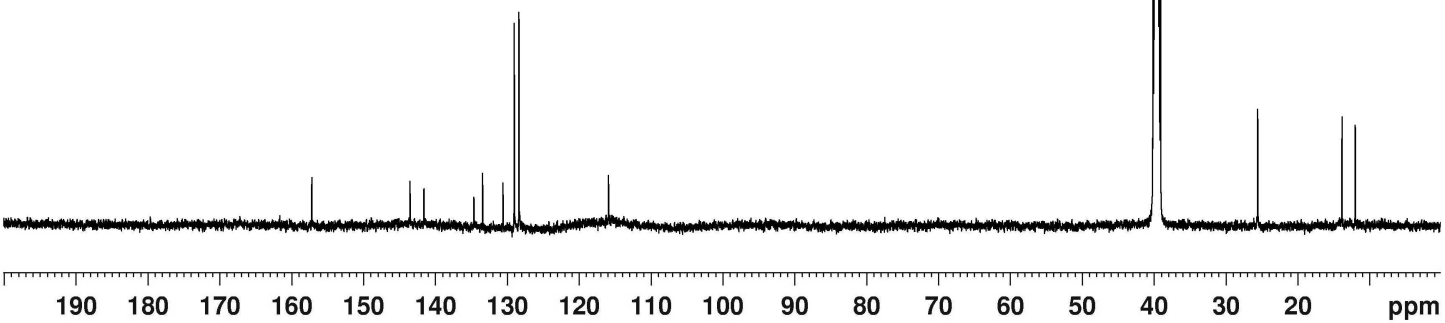




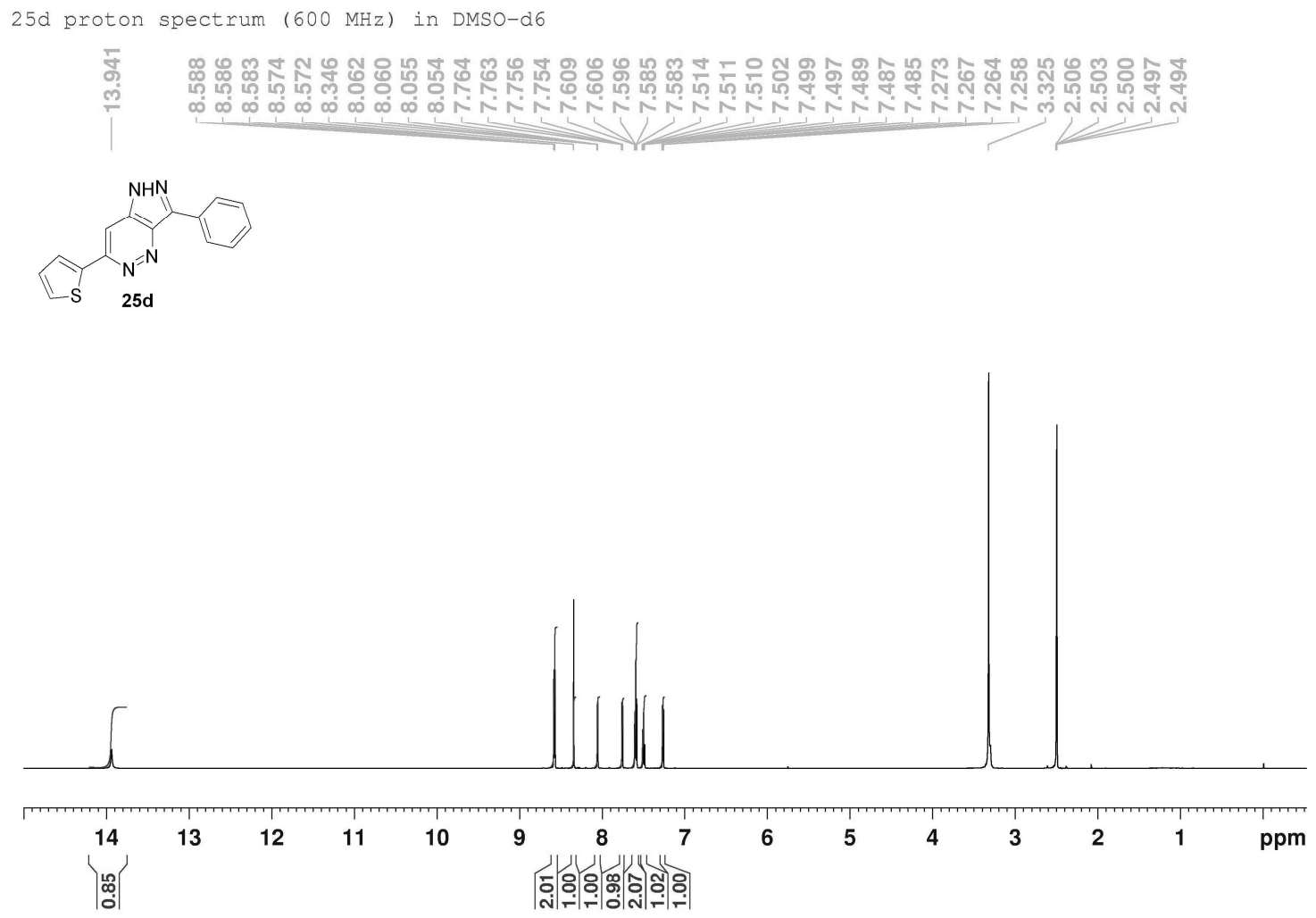

25d carbon spectrum (150 MHz) in DMSO-d6

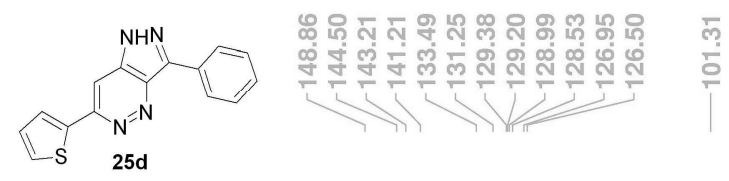

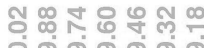

ㅇํำ

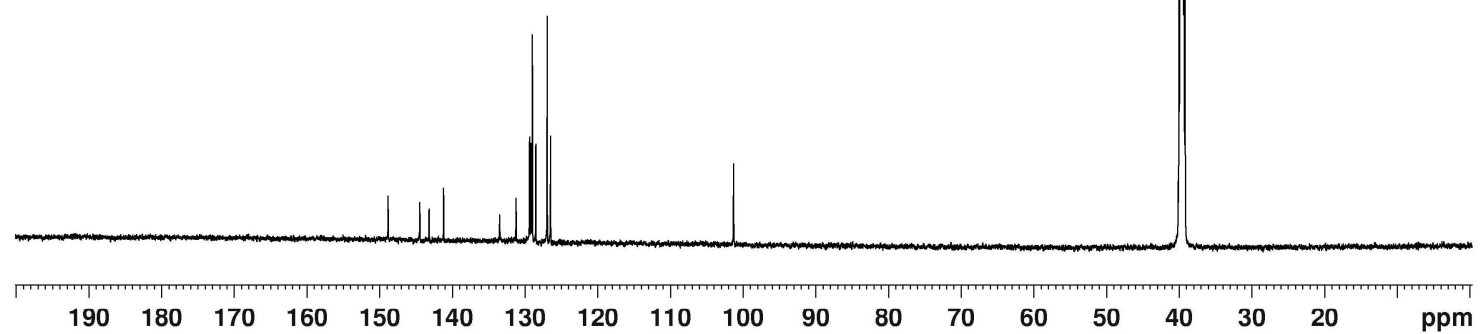


25 e proton spectrum (600 MHz) in DMSO-d6

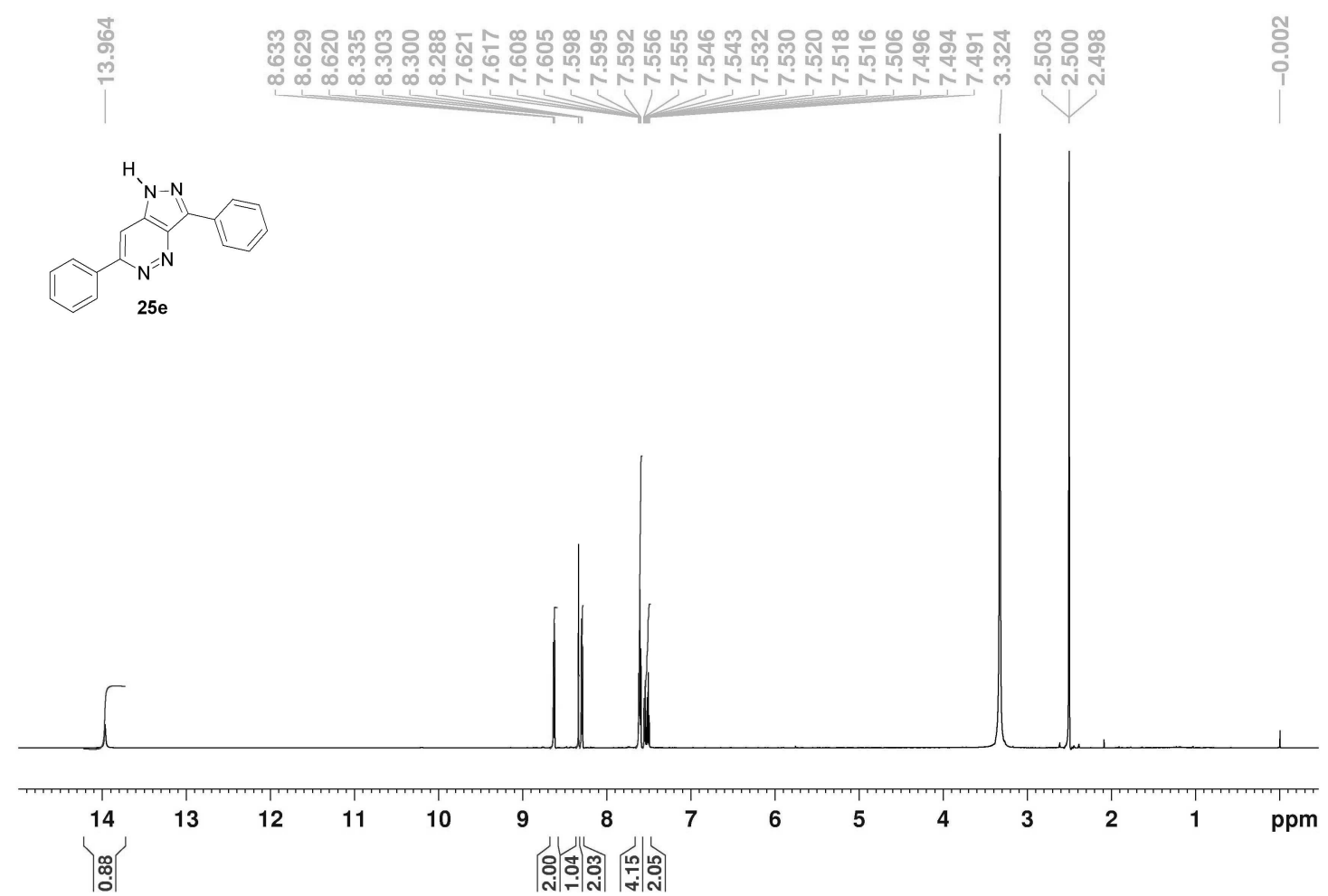

25 e carbon spectrum ( $150 \mathrm{MHz}$ ) in DMSO-d6

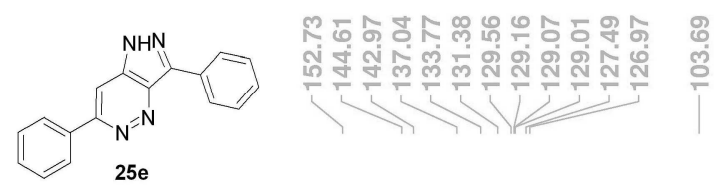

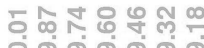

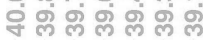

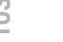


$25 \mathrm{f}$ proton spectrum $(600 \mathrm{MHz})$ in DMSO-d6

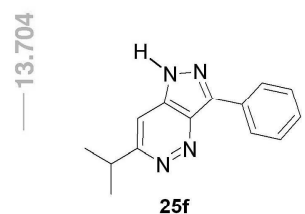

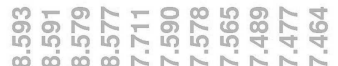

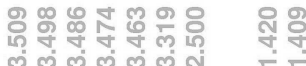

$25 f$ $\infty \infty \infty \infty N+N \mid N$
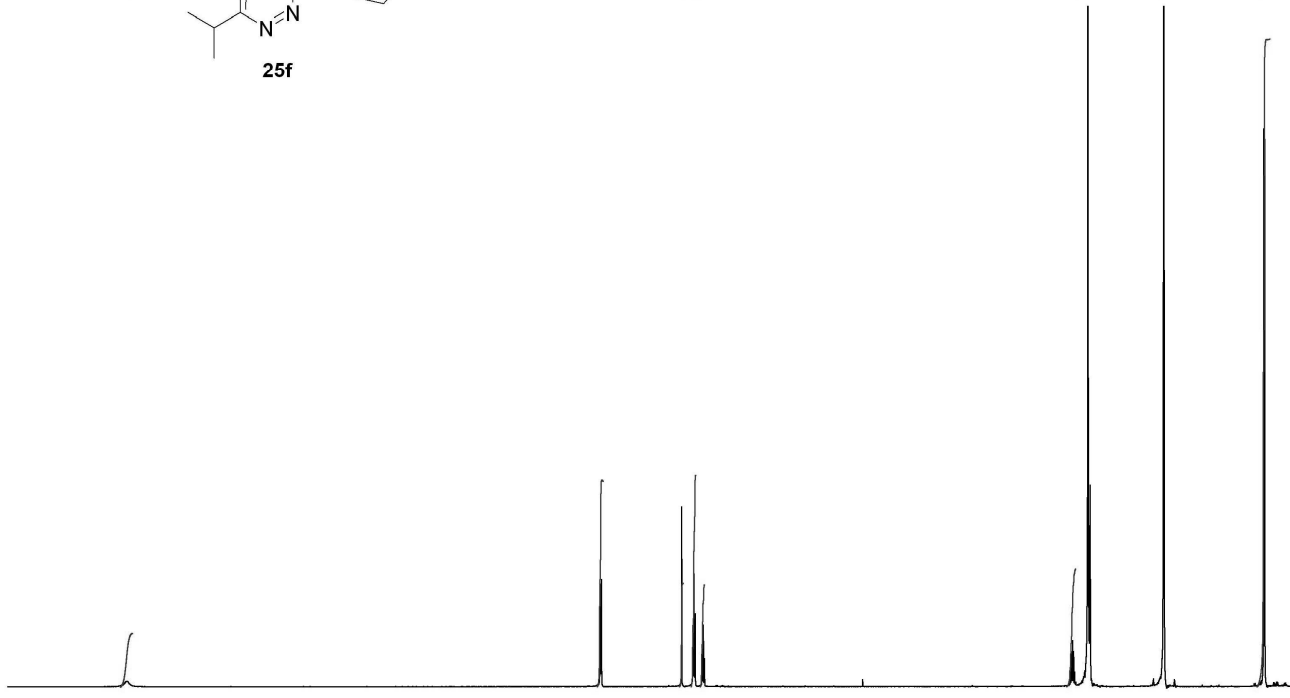

$14 \quad 13 \quad 12$ (ฟ్)

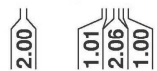

4 ?ִ.

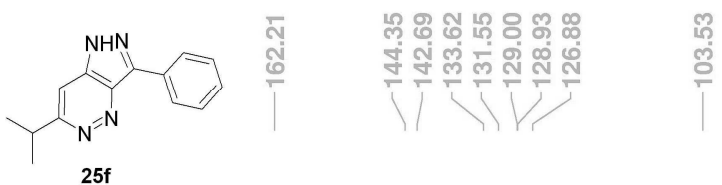

충ํำ

$25 f$

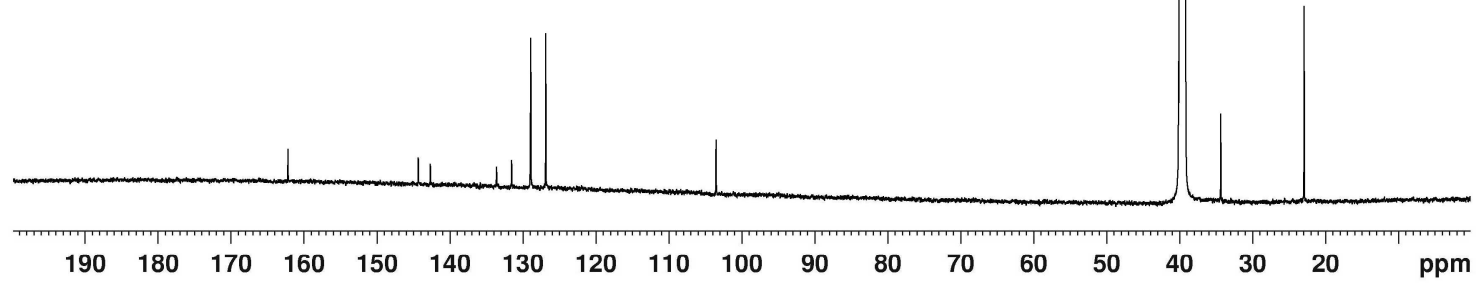




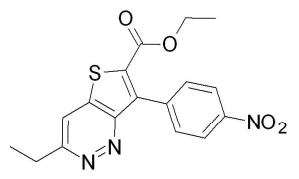

26a

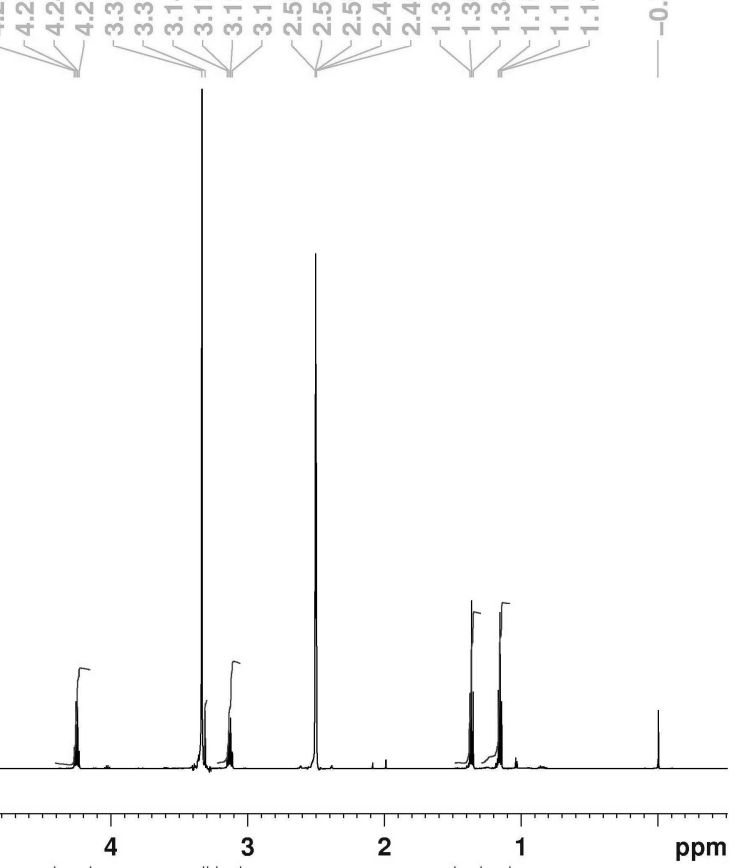

㐭学

|

|ฺฺָָ

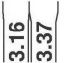

26a carbon spectrum (150 MHz) in DMSO-d6

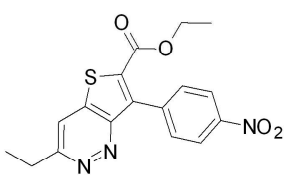

$26 a$

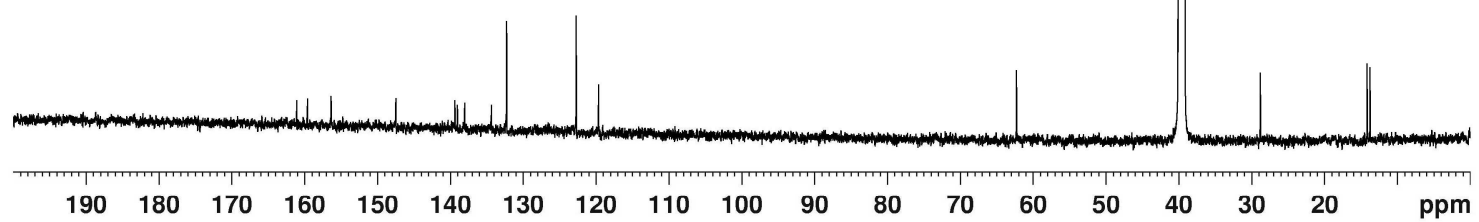


26b proton spectrum (600 $\mathrm{MHz}$ ) in $\mathrm{CDCl3}$

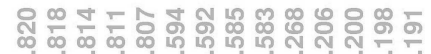

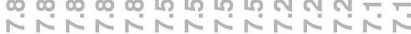

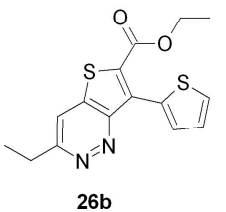

$26 \mathrm{~b}$

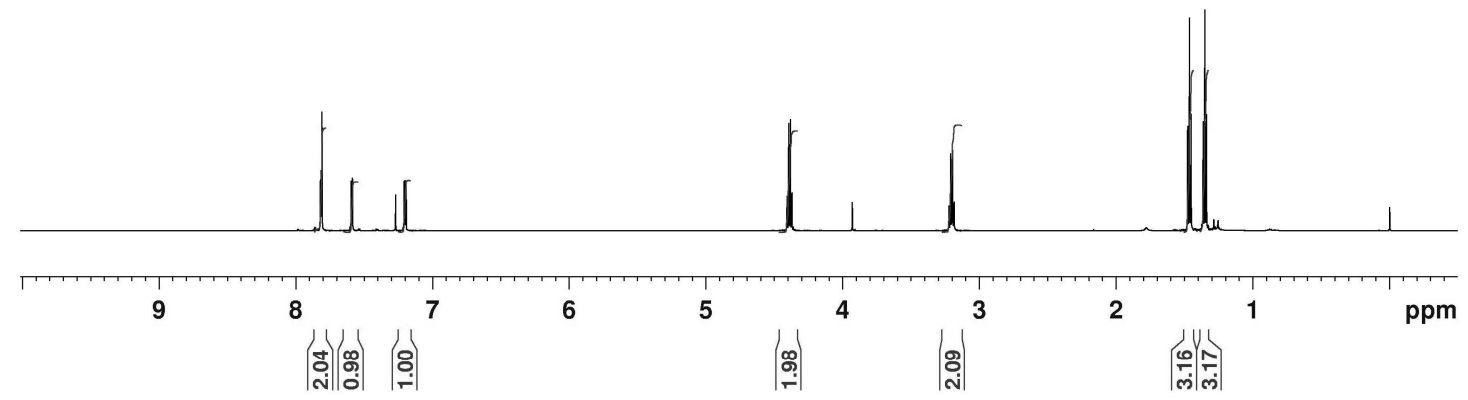

$26 \mathrm{~b}$ carbon spectrum (150 $\mathrm{MHz}$ ) in $\mathrm{CDCl} 3$

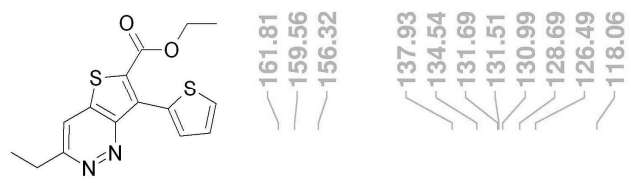

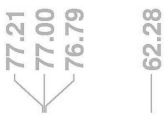

$26 b$

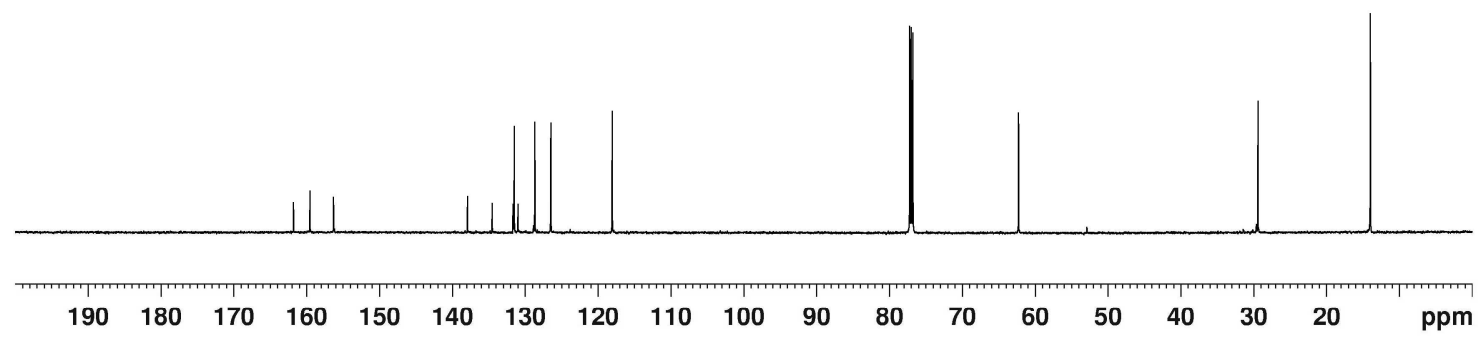




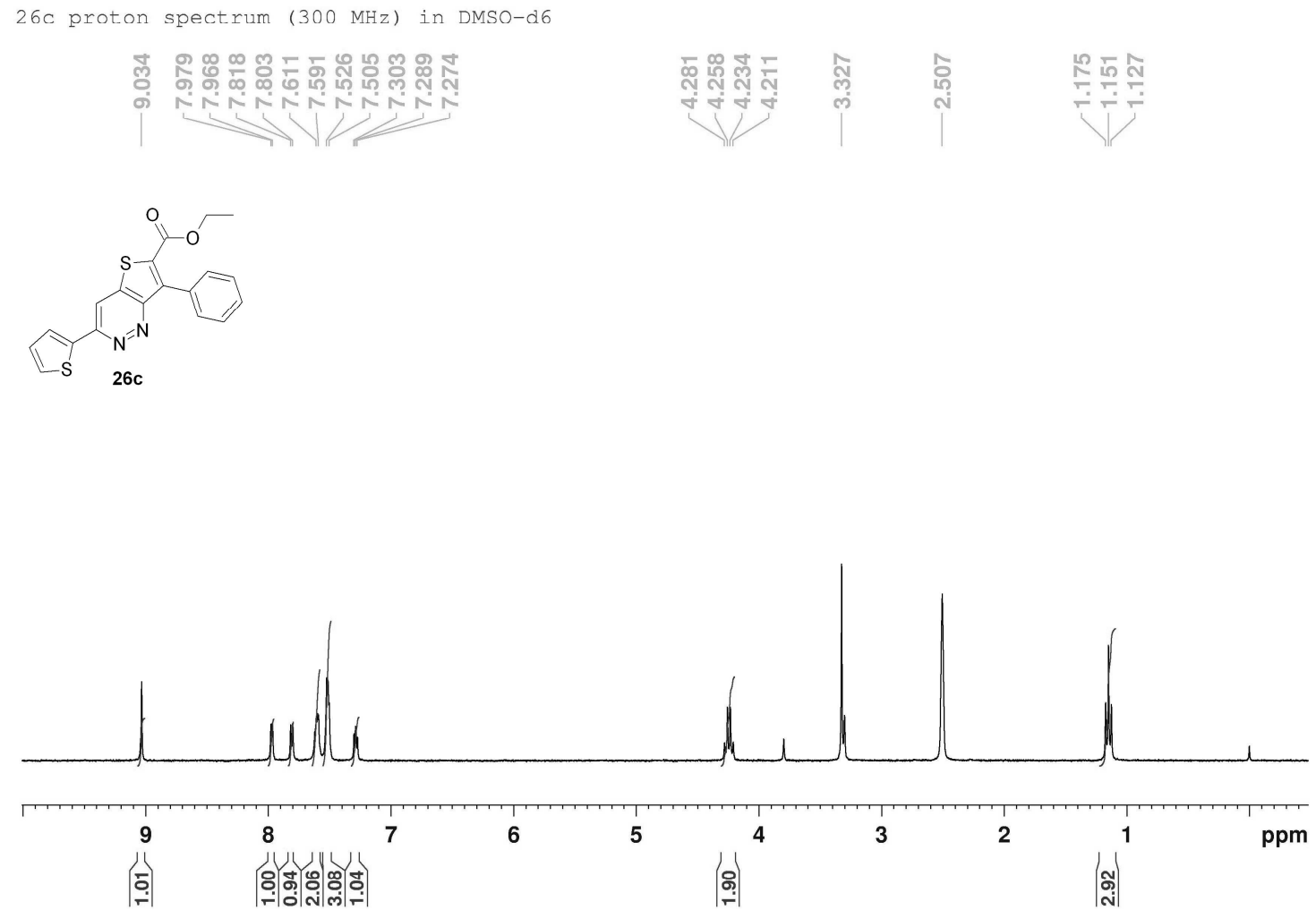

$26 \mathrm{c}$ carbon spectrum ( $75 \mathrm{MHz}$ ) in DMSO-d6
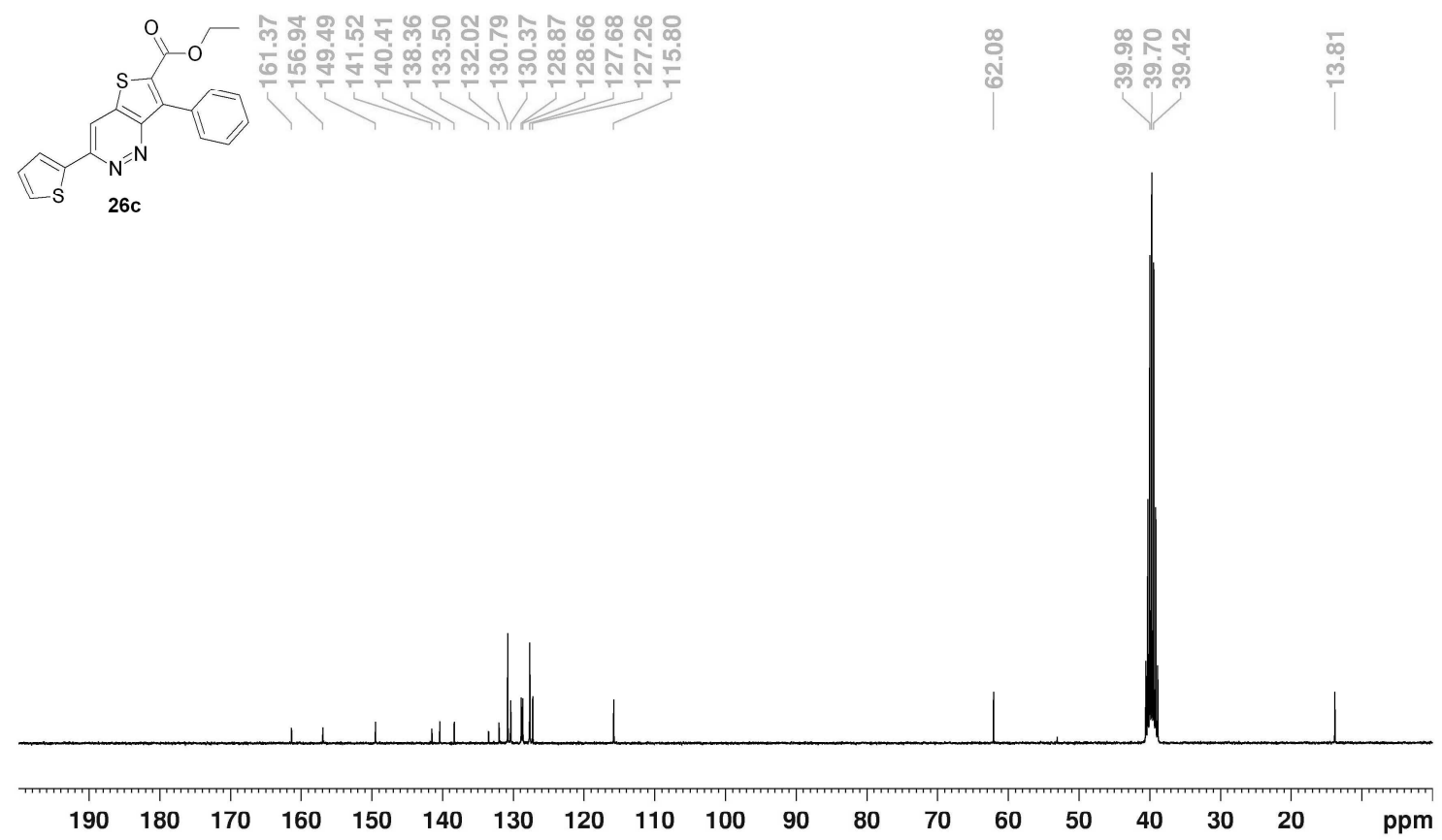
$26 \mathrm{~d}$ proton spectrum $(600 \mathrm{MHz})$ in DMSO-d6
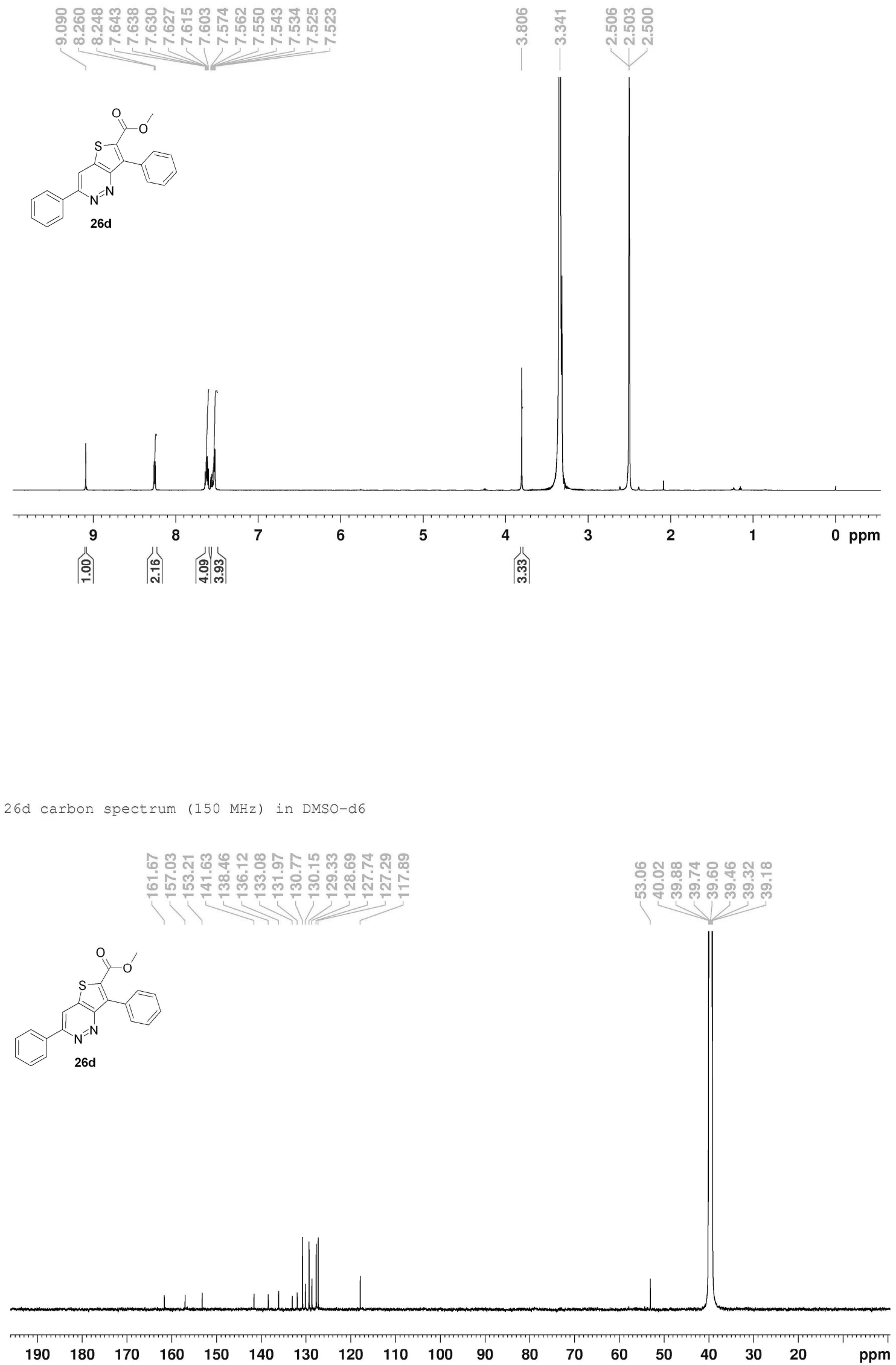


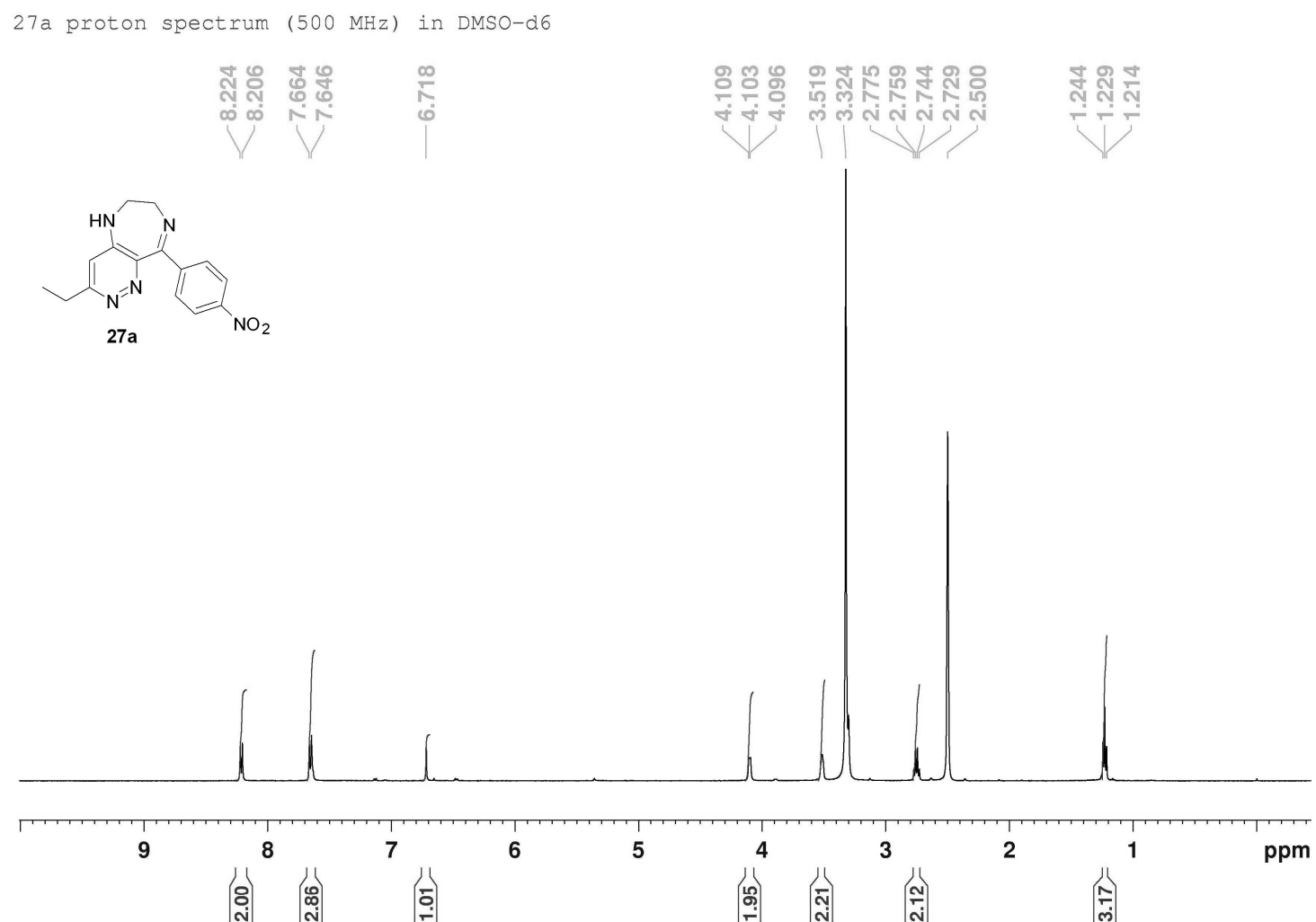

27a carbon spectrum ( $125 \mathrm{MHz}$ ) in DMSO-d6

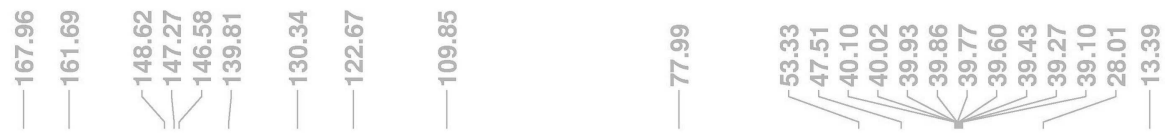
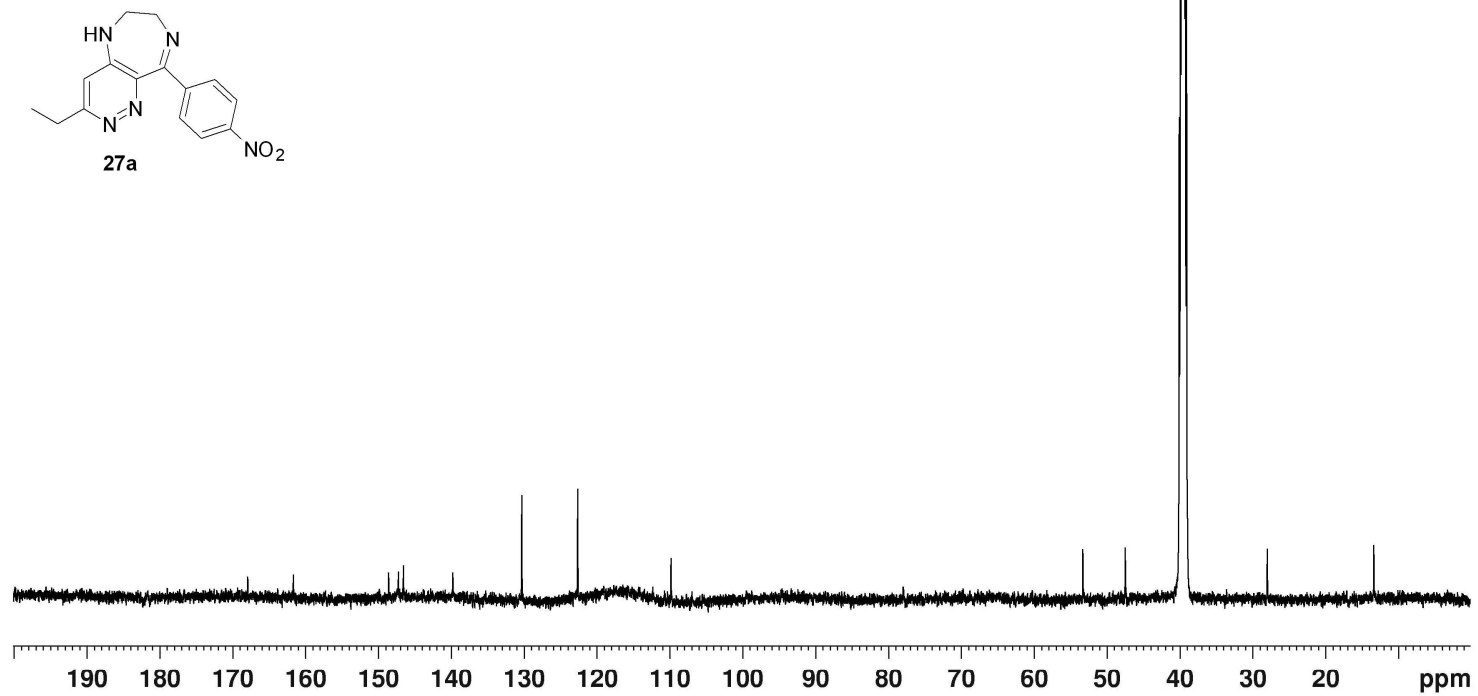


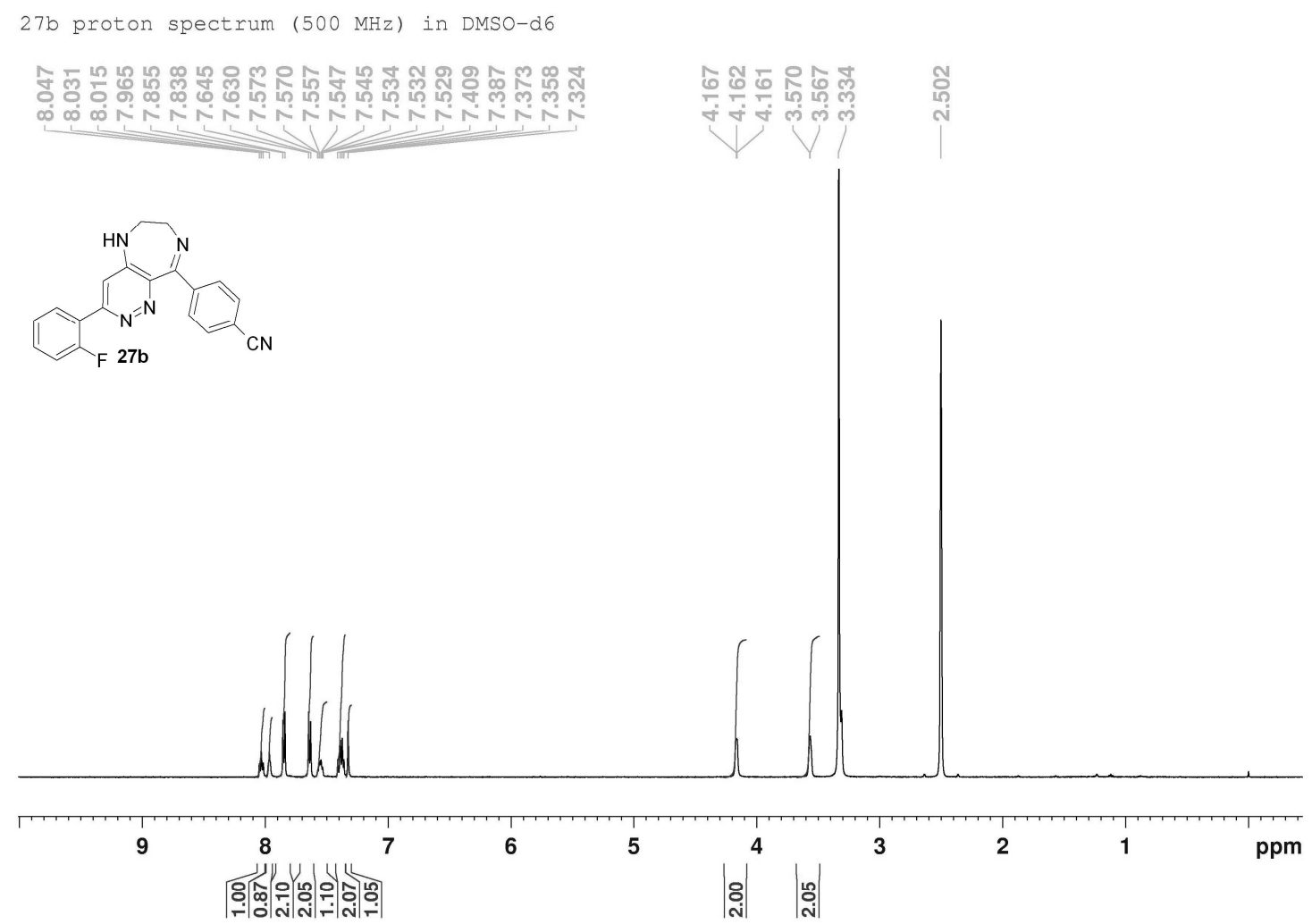

27b carbon spectrum (125 MHz) in DMSO-d6

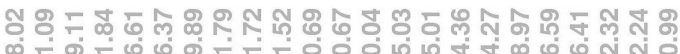

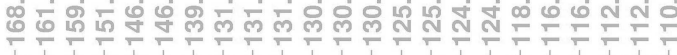

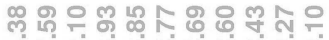

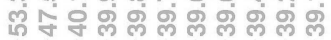
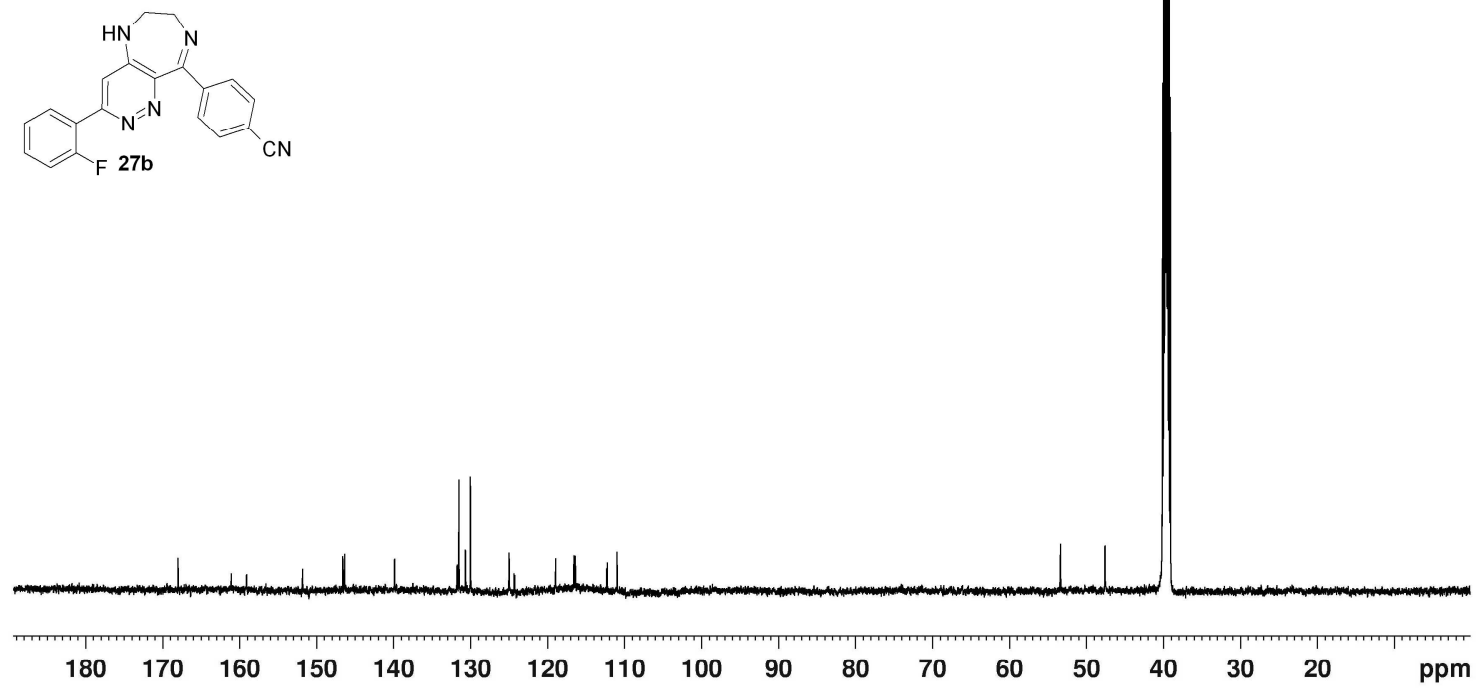


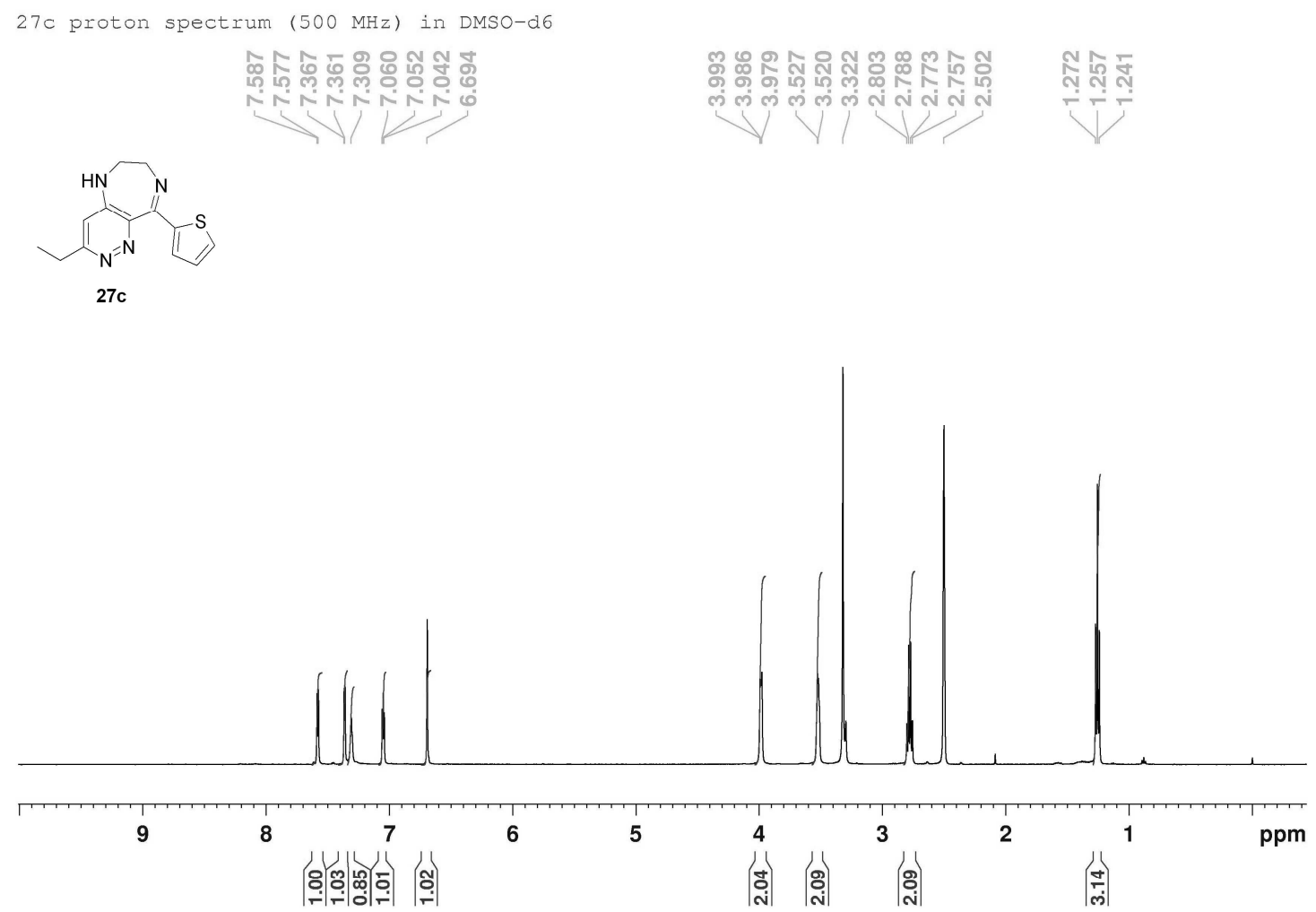

27c carbon spectrum (125 MHz) in DMSO-d6
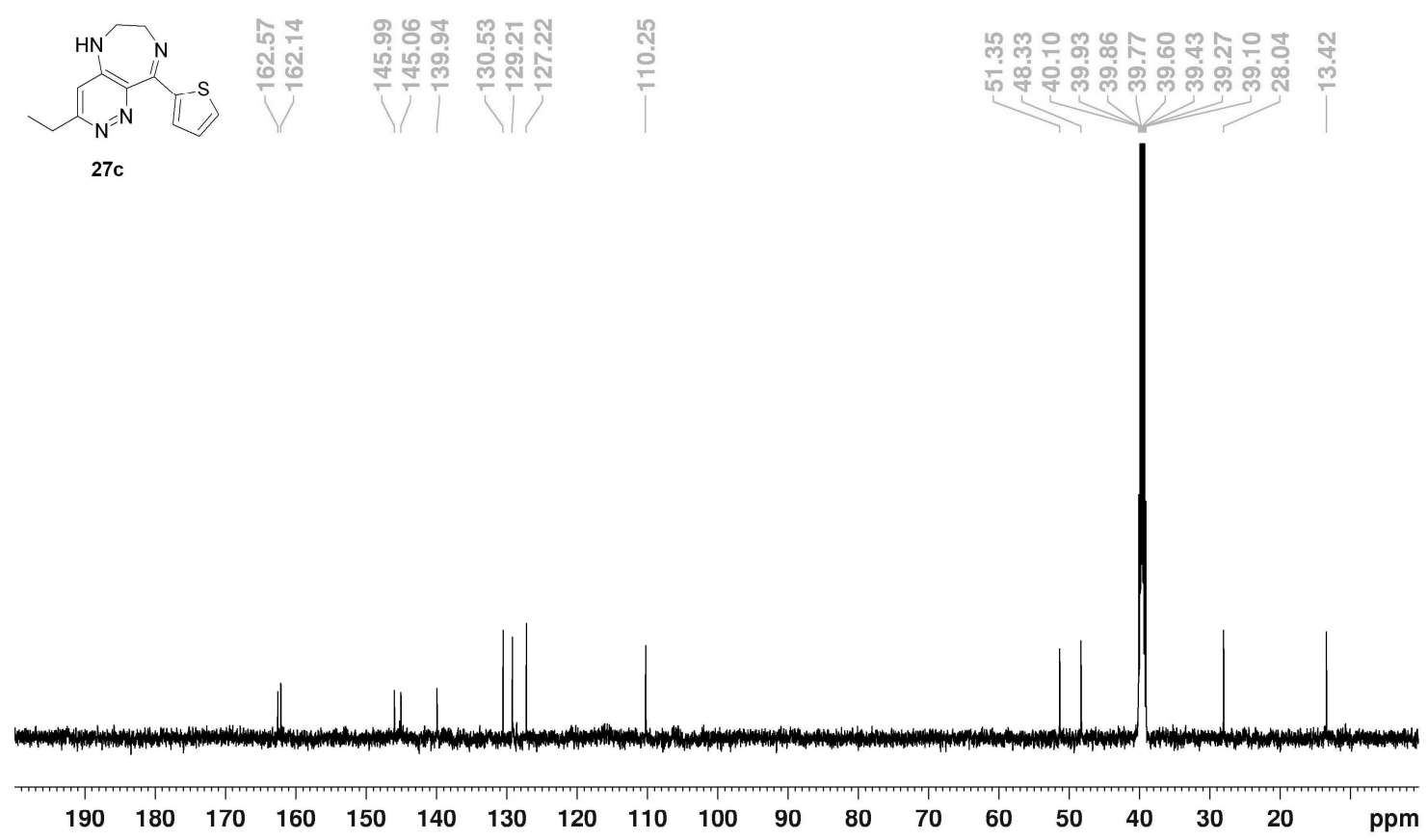
27d proton spectrum (600 MHz) in DMSO-d6

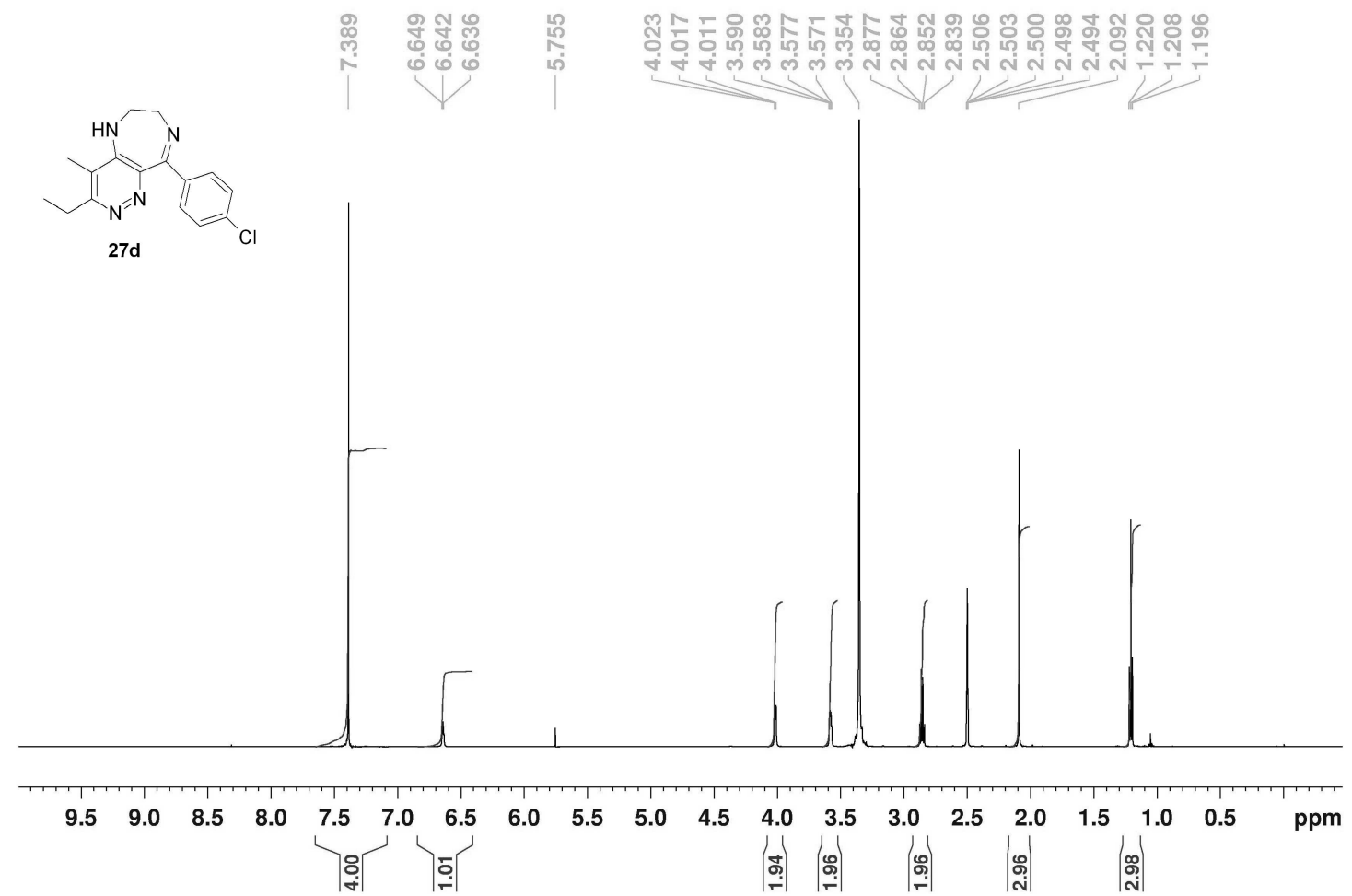

27d carbon spectrum (150 MHz) in DMSO-d6

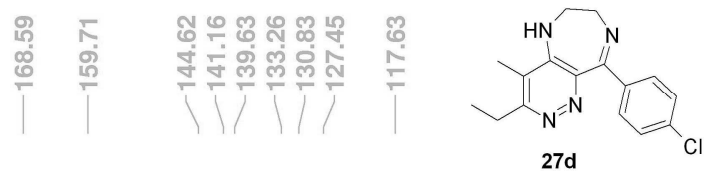

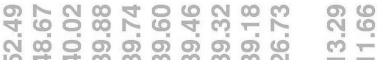

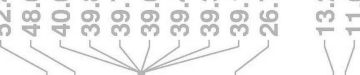

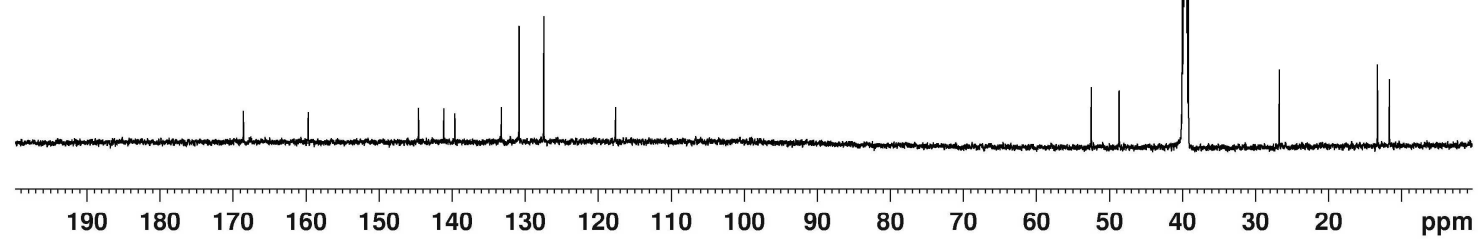


27 e proton spectrum (600 $\mathrm{MHz}$ ) in DMSO-d6

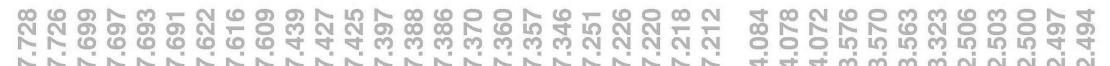
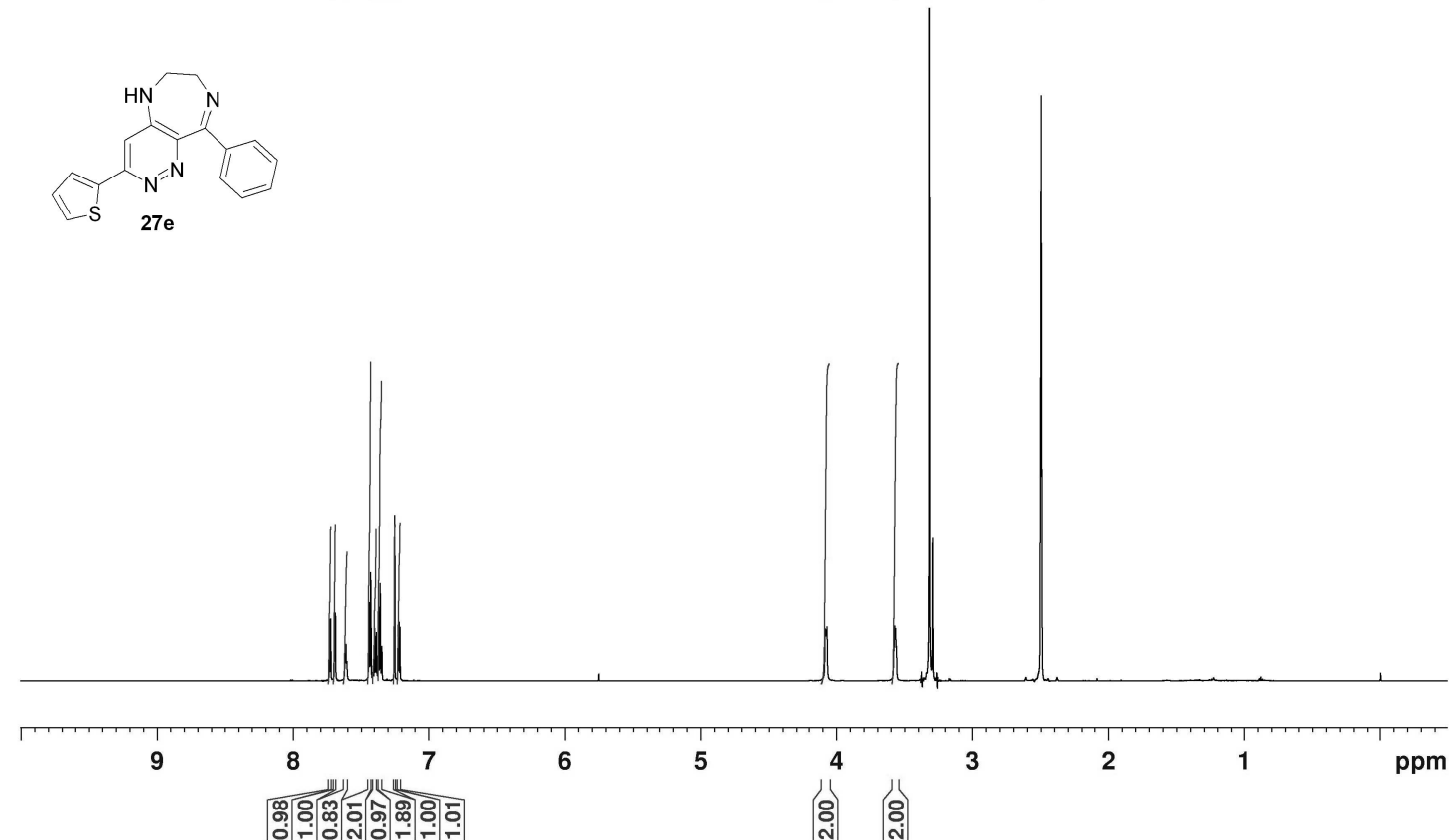

27 p proton spectrum (150 MHz) in DMSO-d6
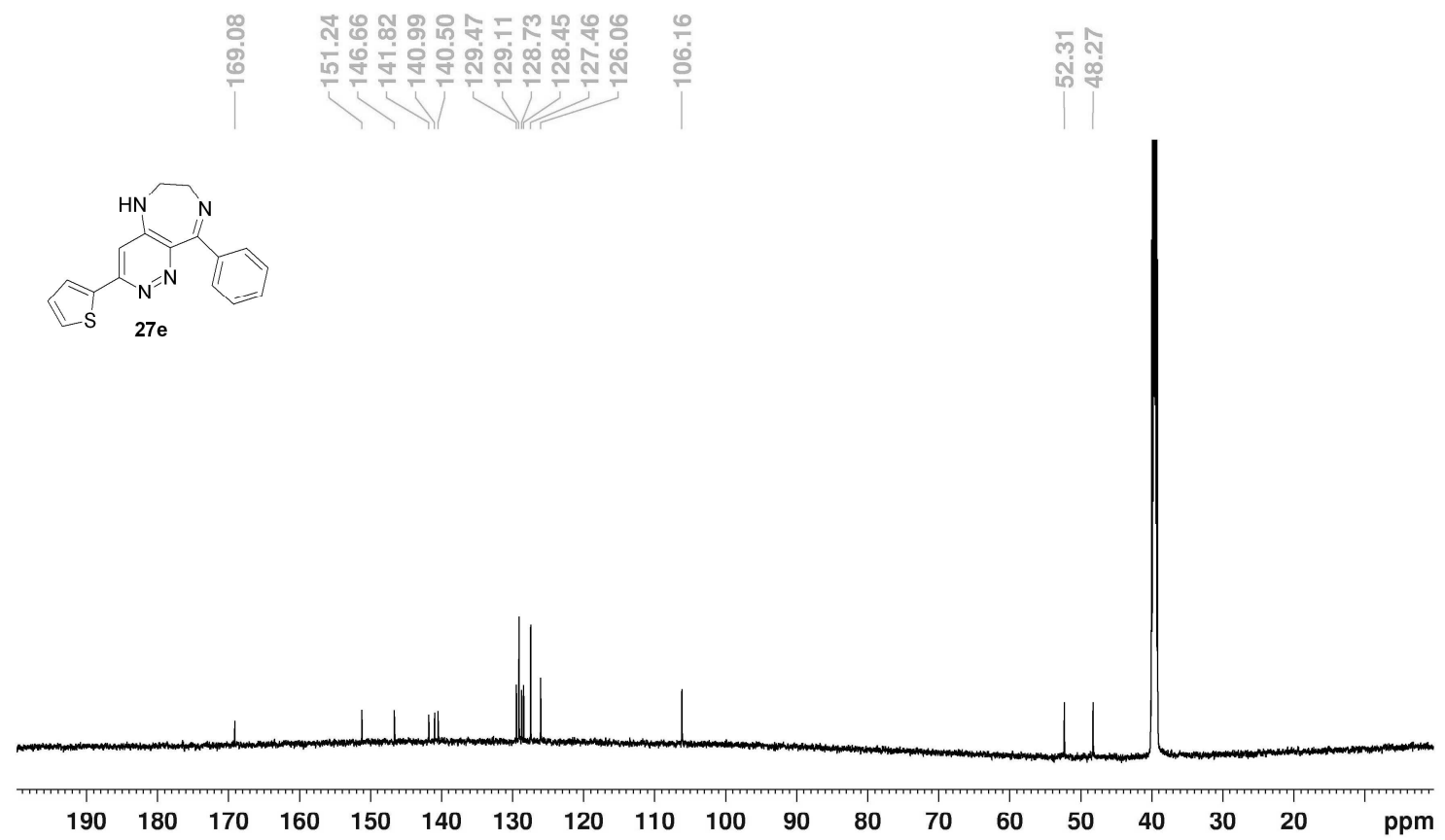

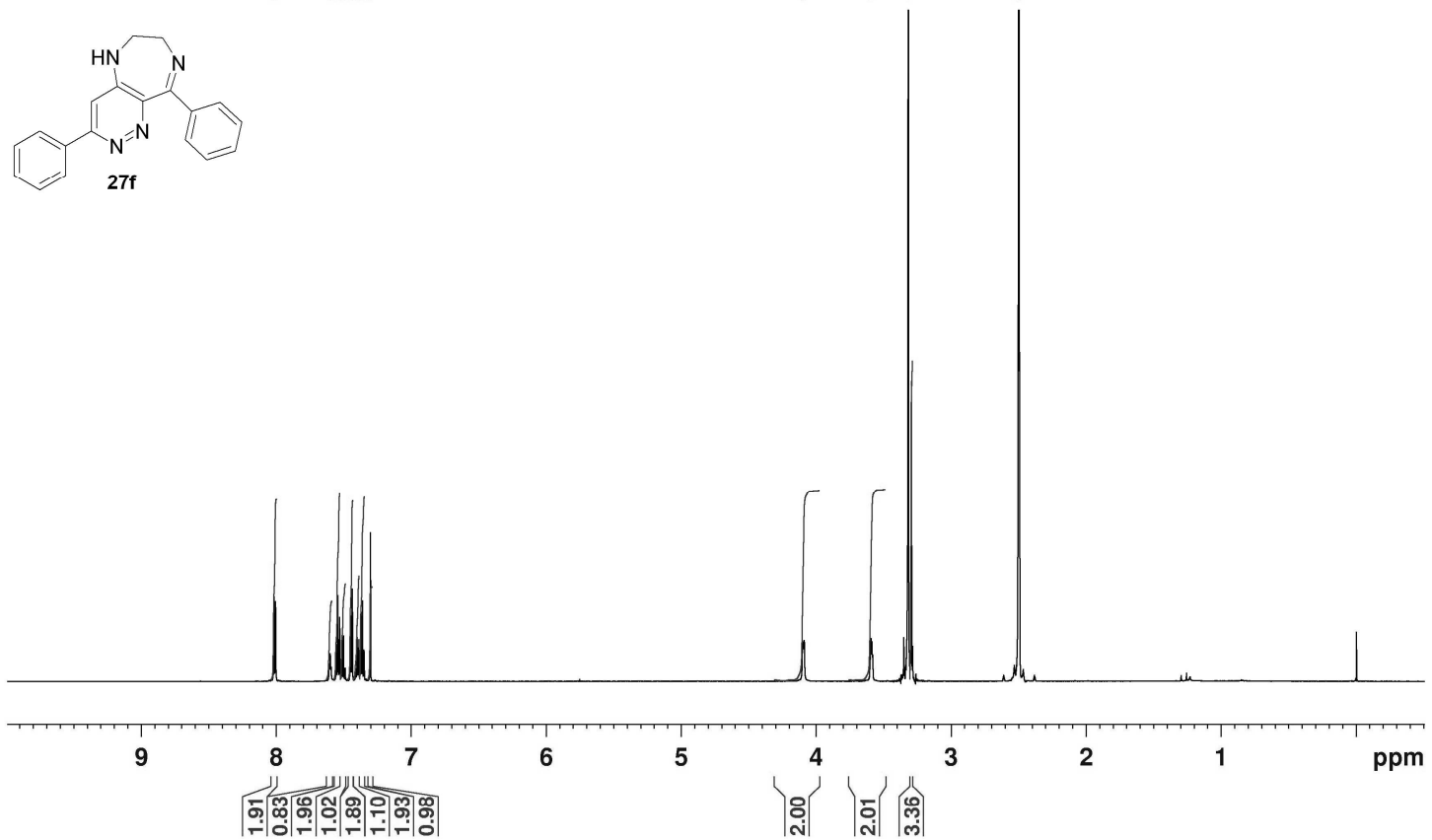

$27 \mathrm{f}$ carbon spectrum (150 MHz) in DMSO-d6
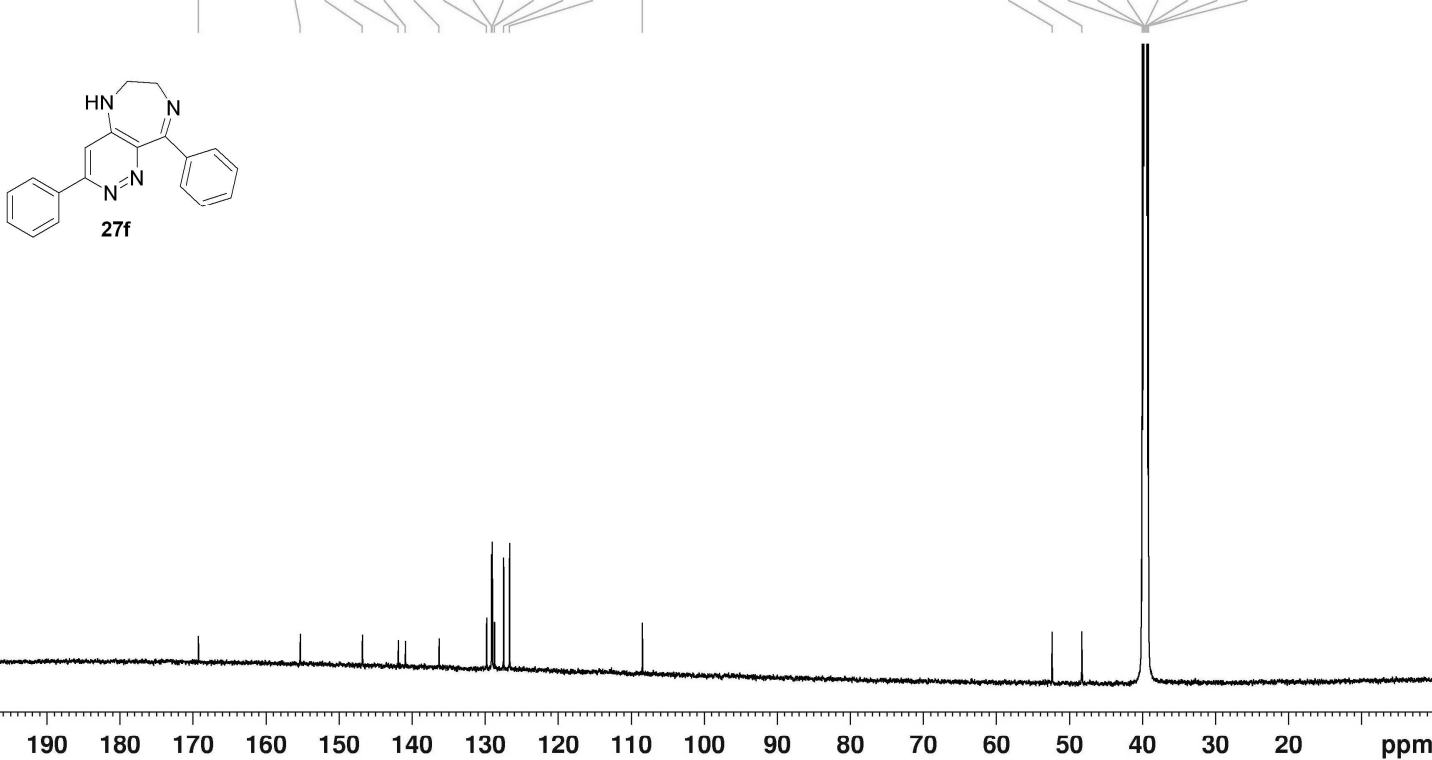
$27 \mathrm{~g}$ proton spectrum ( $500 \mathrm{MHz}$ ) in DMSO-d6

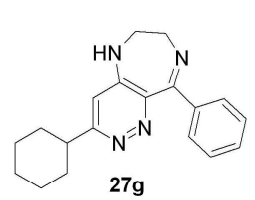

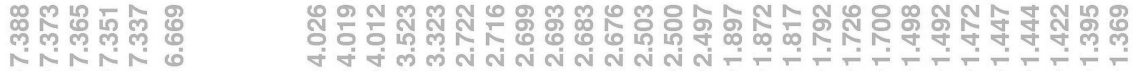

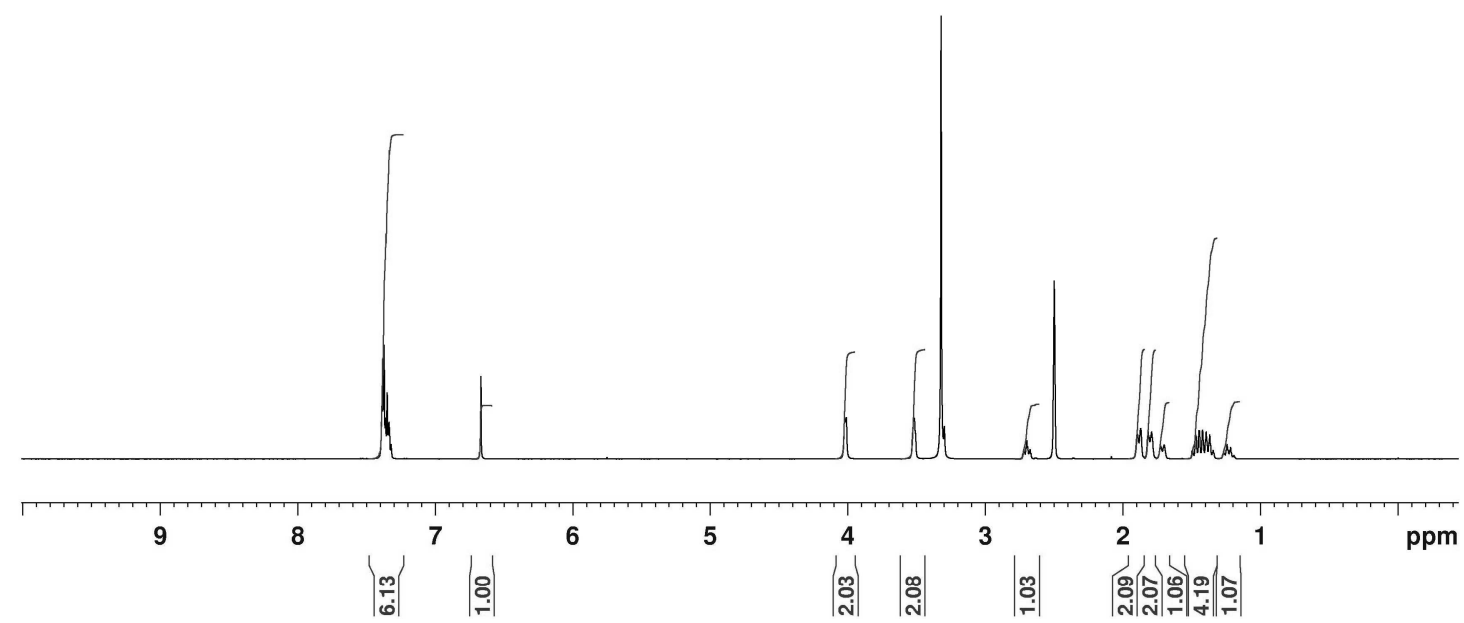

$27 \mathrm{~g}$ carbon spectrum (125 MHz) in DMSO-d6
ले ले

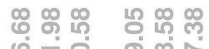

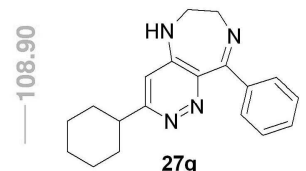

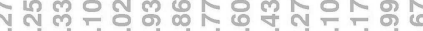

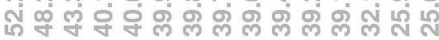

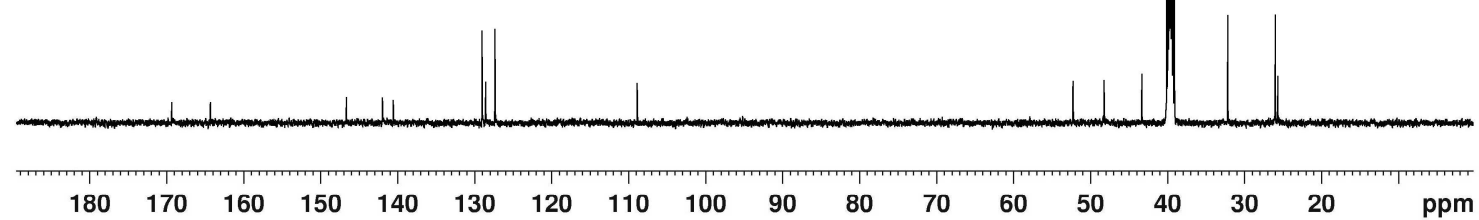


$27 \mathrm{~h}$ proton spectrum ( $500 \mathrm{MHz})$ in DMSO-d6
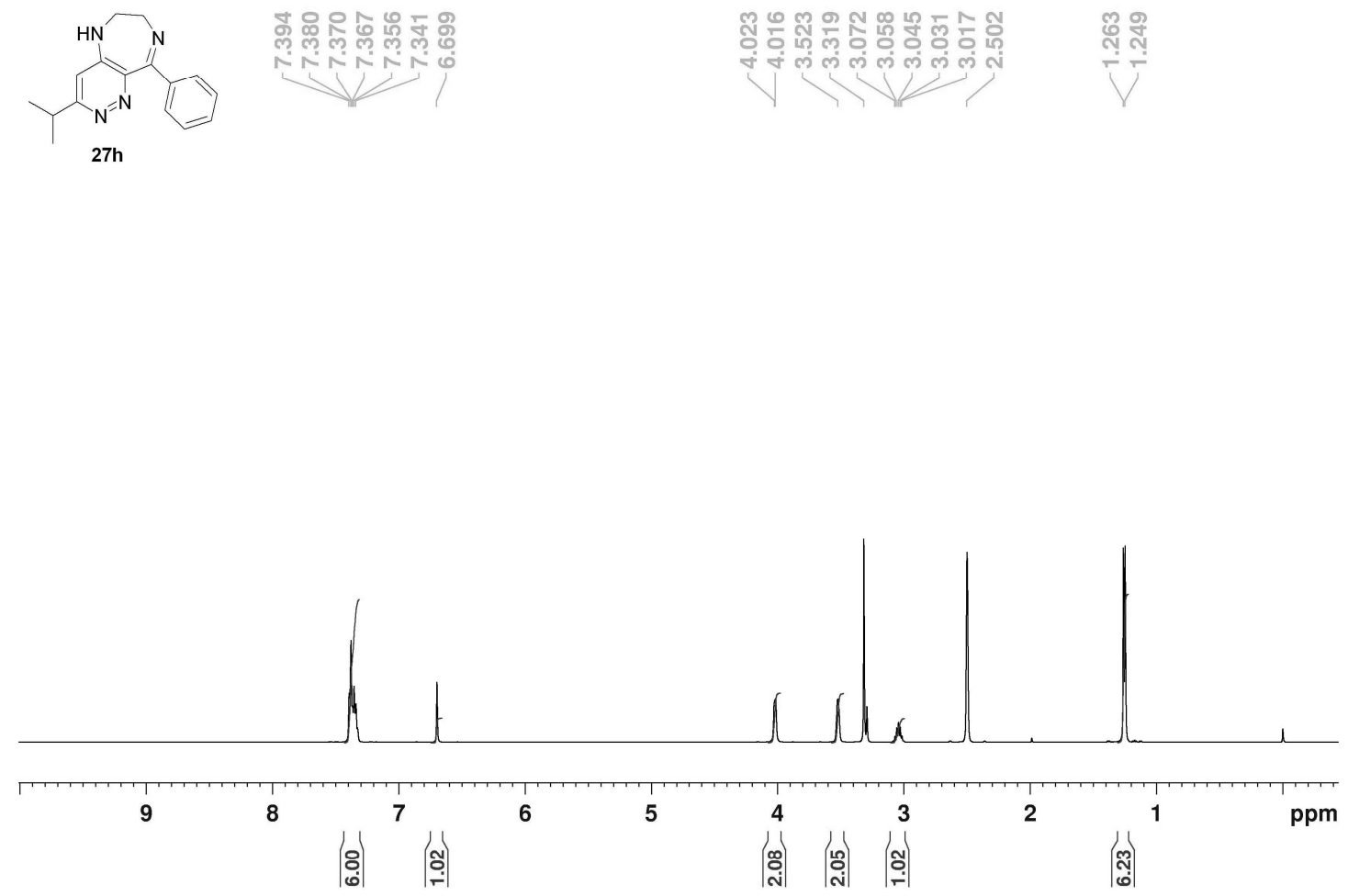

$27 \mathrm{~h}$ carbon spectrum (125 $\mathrm{MHz}$ ) in DMSO-d6
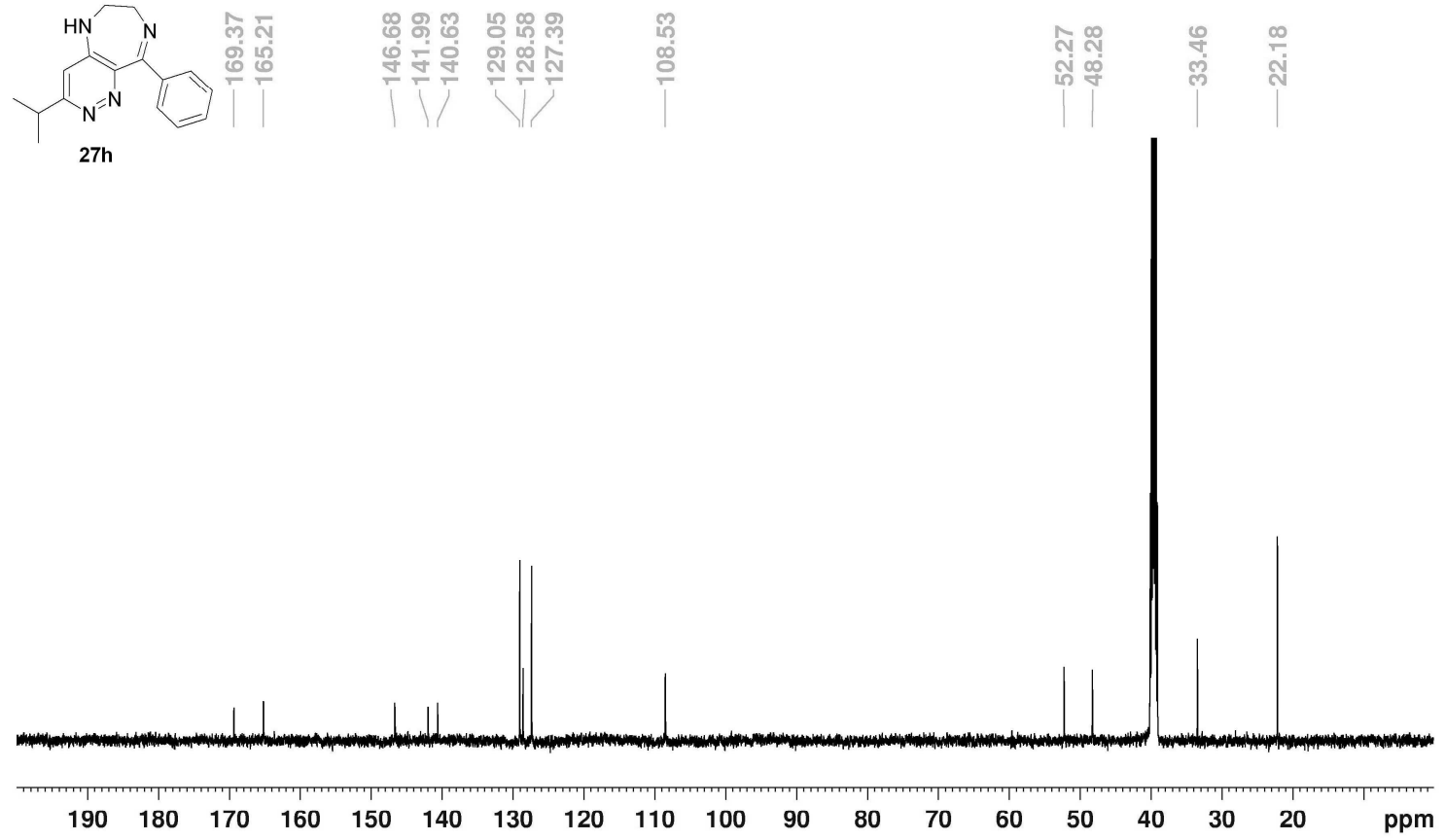


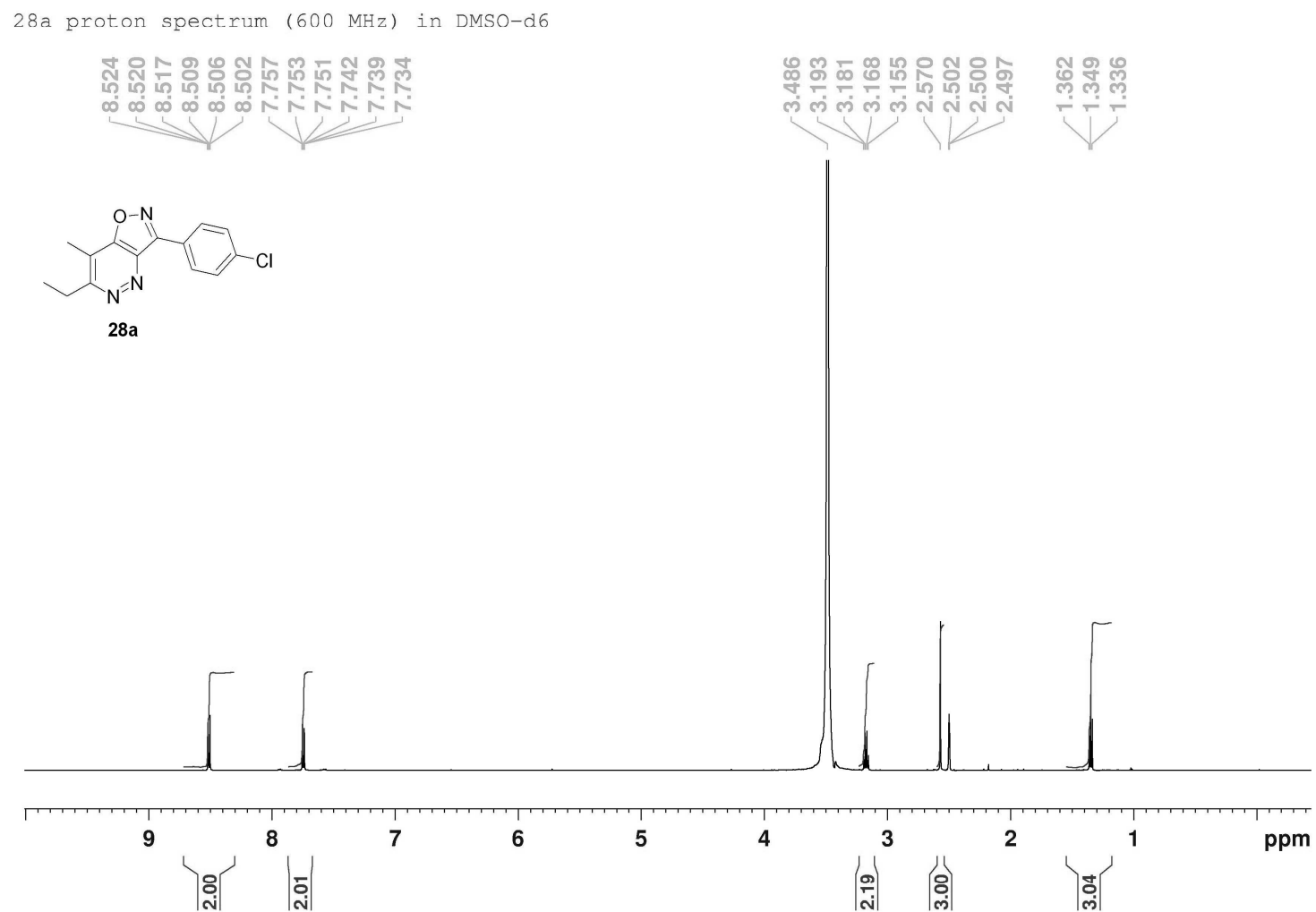

28a carbon spectrum (150 MHz) in DMSO-d6
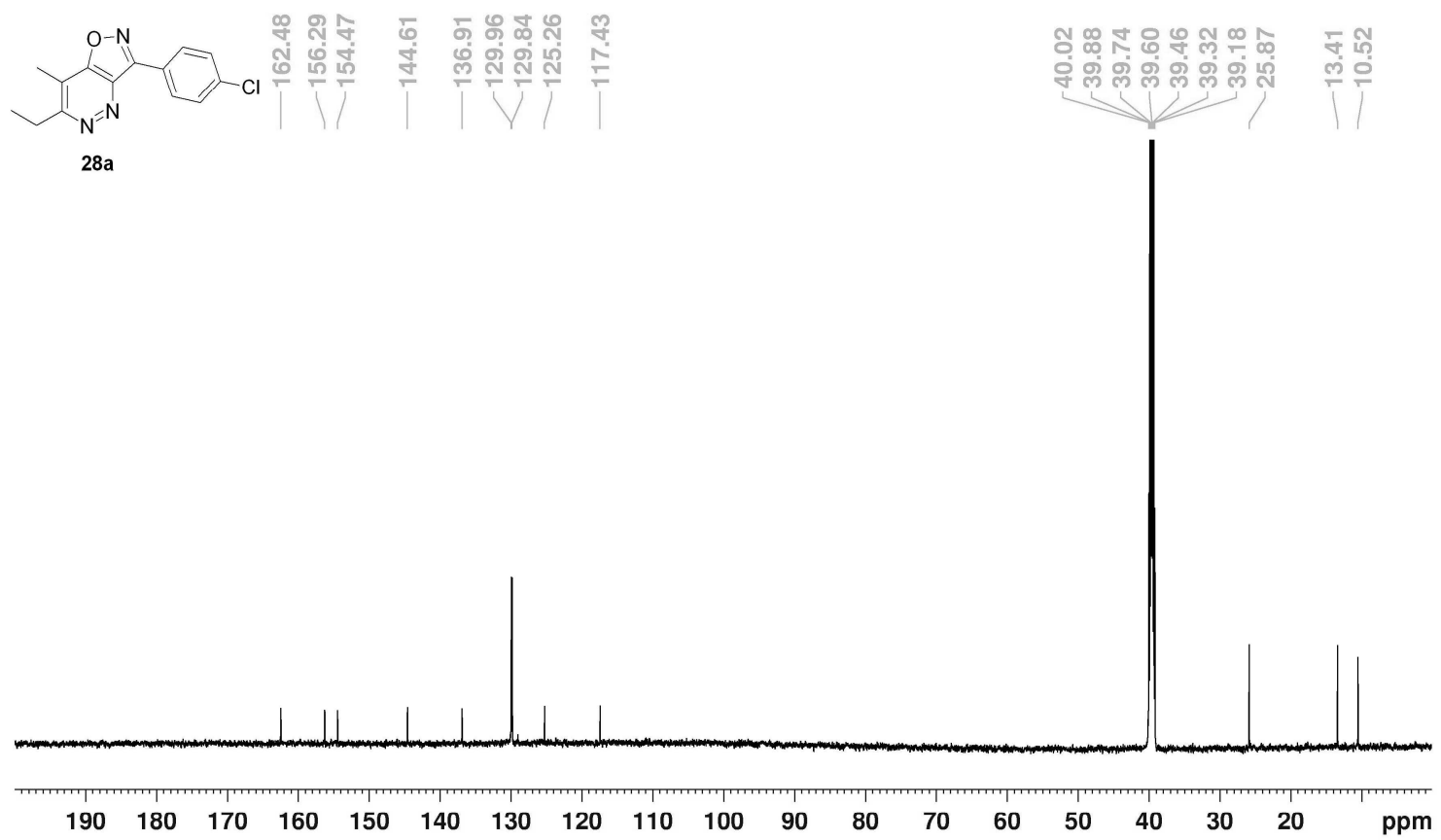


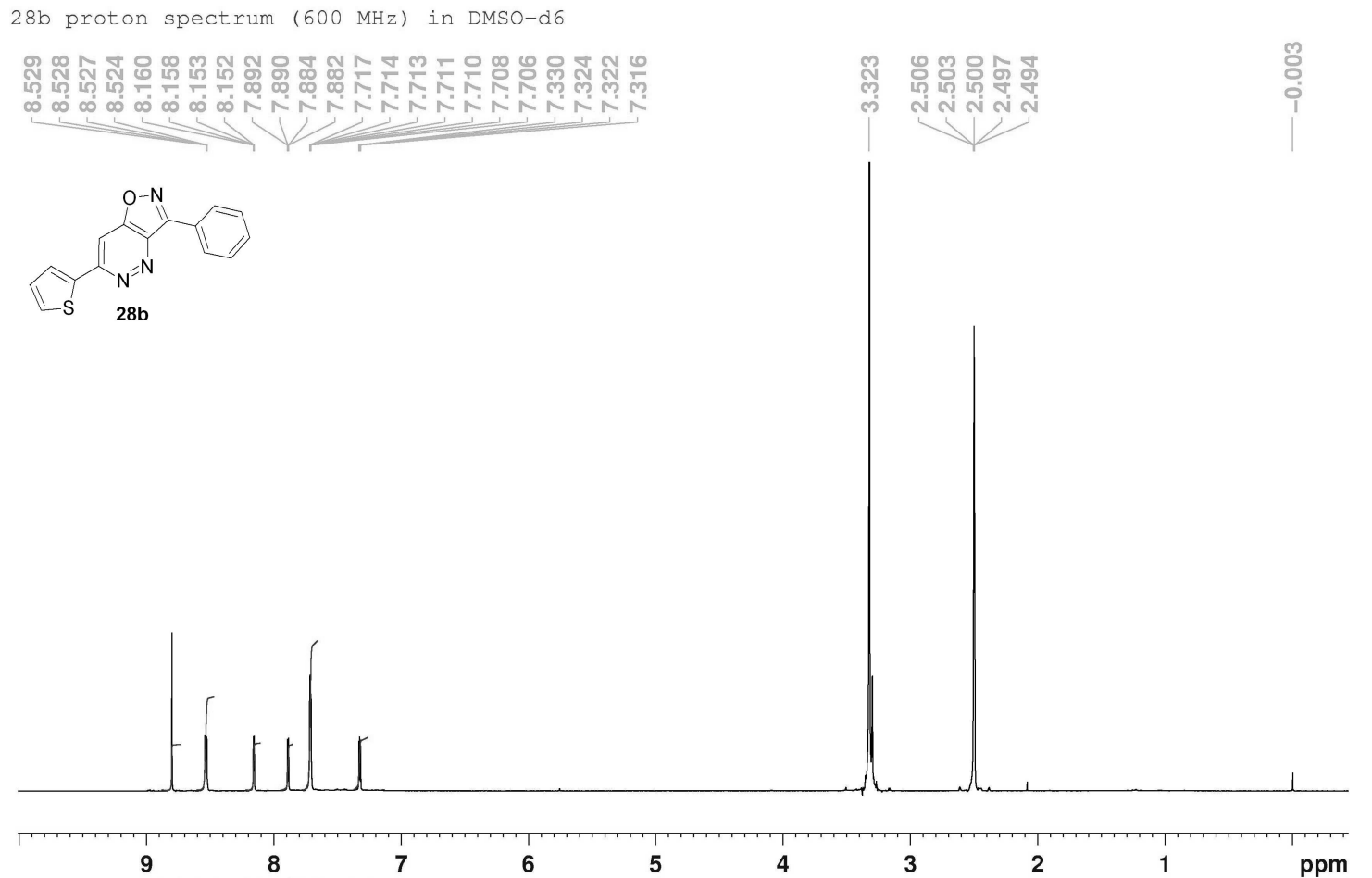

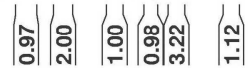

28.b carbon spectrum (150 $\mathrm{MHz})$ in DMSO-d6

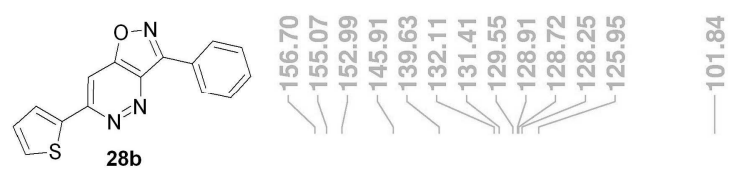

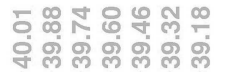

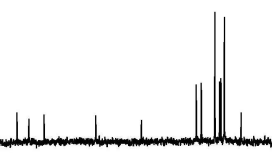

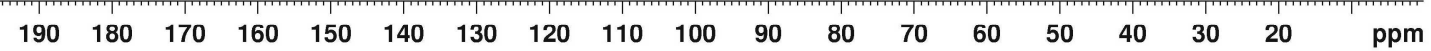


<smiles>CCc1cc(O)c(C(=O)c2ccc([N+](=O)[O-])cc2)nn1</smiles>

24b

UV Detector: TIC

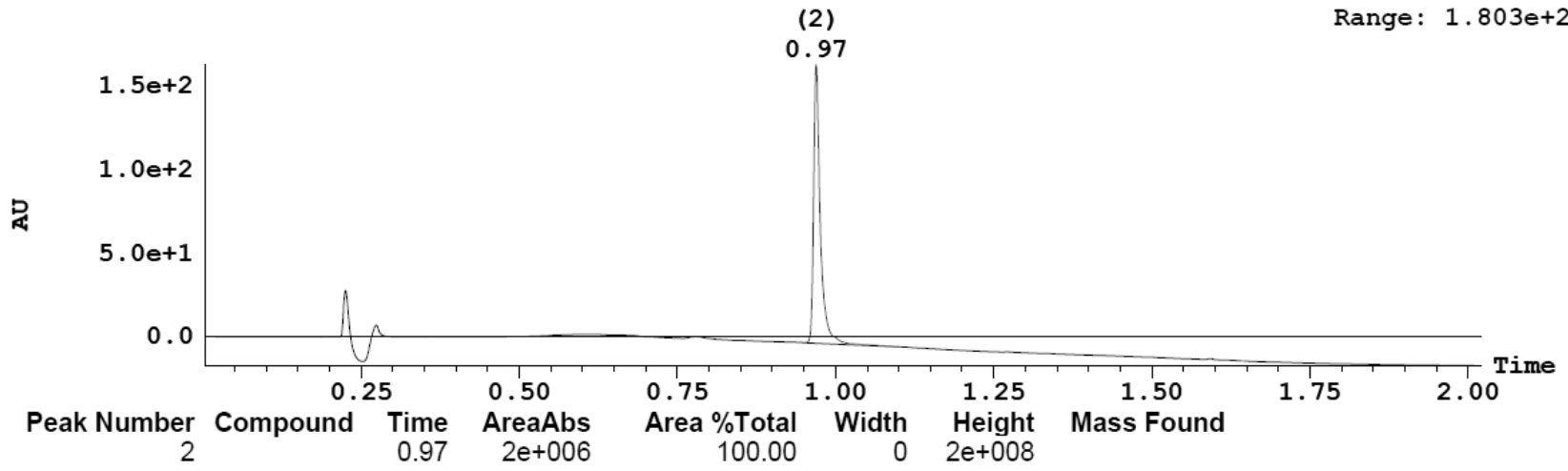

Peak ID Compound Time Mass Found

$\begin{array}{ll} & \\ & \\ \text { SAMPLE: } & 2: 97 \\ & \text { Combine }(227: 232)\end{array}$
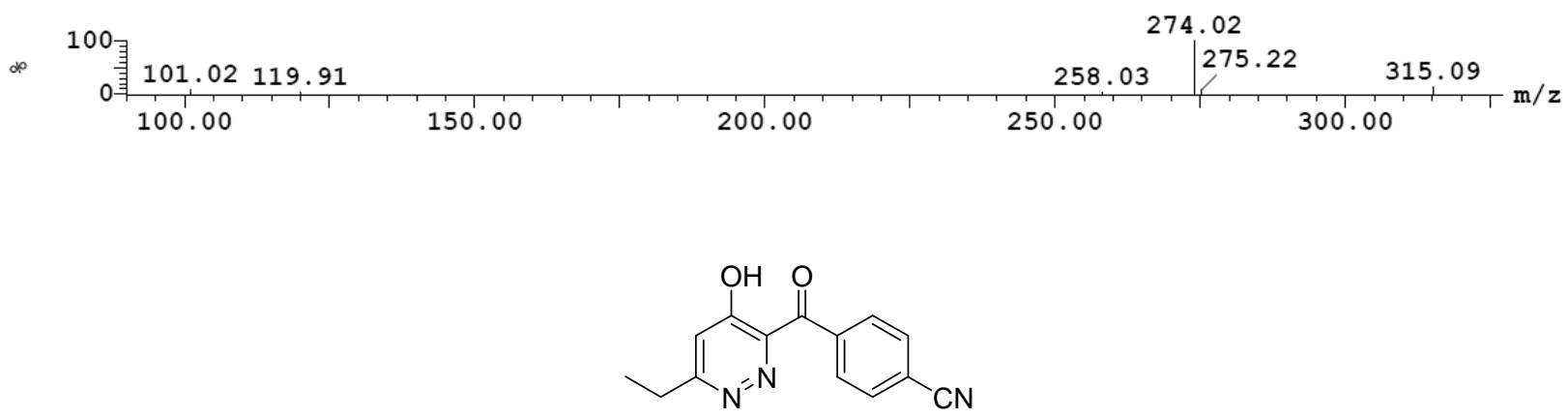

24c

UV Detector: TIC

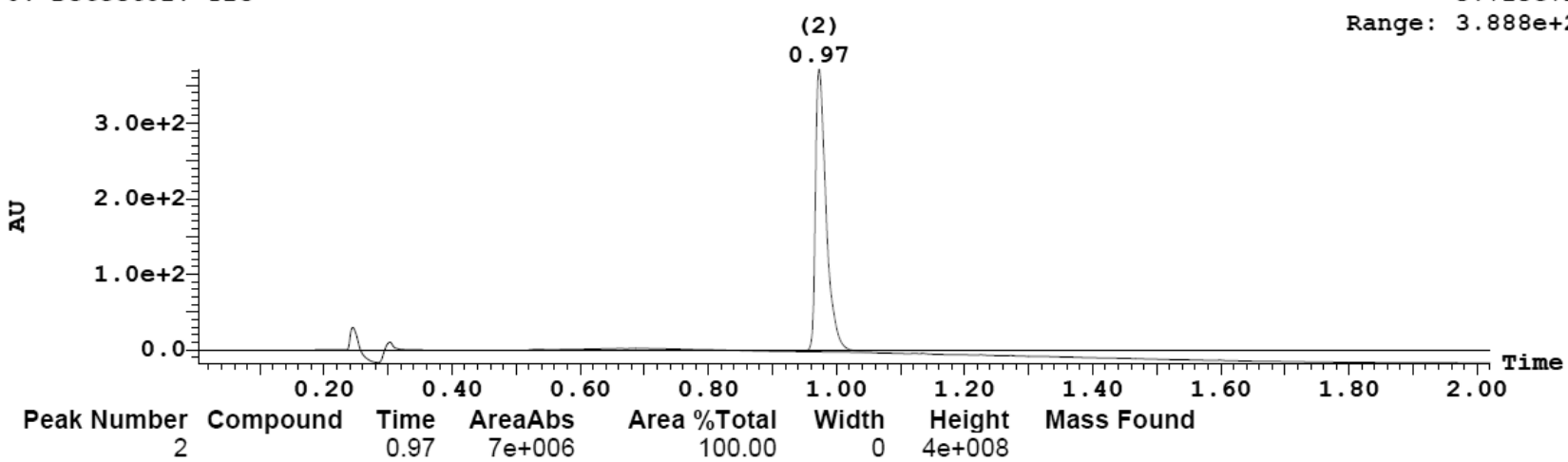

\section{$\begin{array}{rrr}\text { Peak ID Compound Time } & \text { Mass Found } \\ 2 & 0.97 & \end{array}$}

SAMPLE: $1: 7$ Combine (228:233)

do 254.03


<smiles>N#Cc1ccc(C(=O)c2nnc(-c3ccccc3F)cc2O)cc1</smiles>

UV Detector: TIC

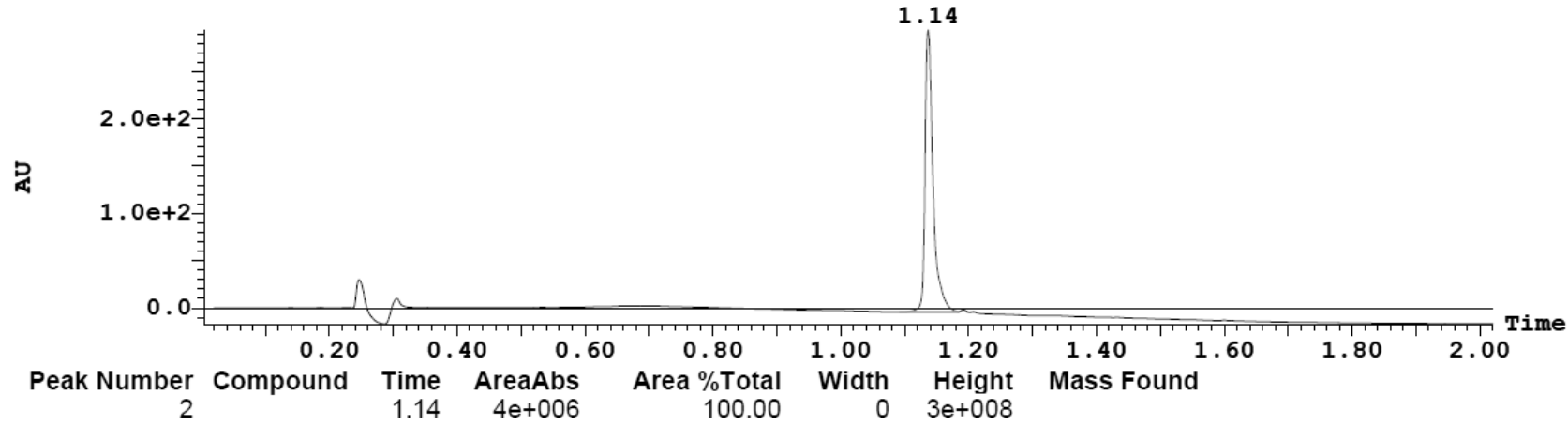

Peak ID Compound Time Mass Found

SAMPLE: $1: 6$ Combine $(267: 272)$

$2.4 e+007$

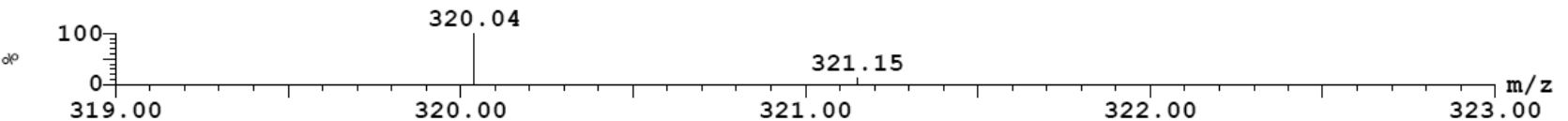<smiles>CCc1cc(O)c(C(=O)c2cccs2)nn1</smiles>

$24 e$

UV Detector: TIC

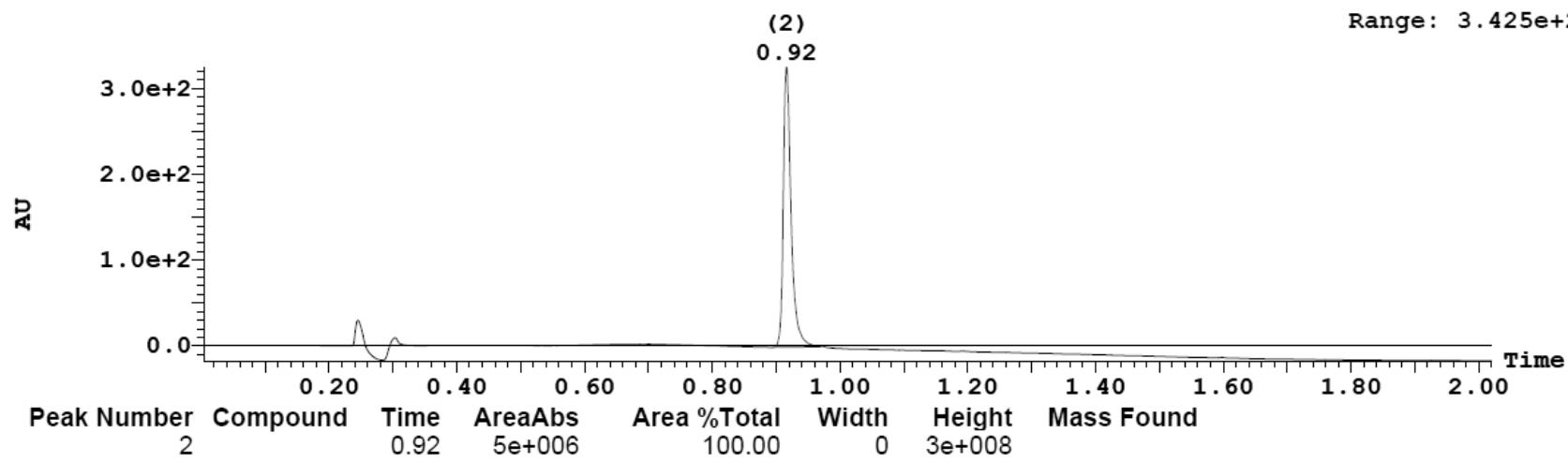

\section{Peak ID Compound Time Mass Found}

SAMPLE: 2:48 Combine $(215: 220)$

$7.7 e+007$

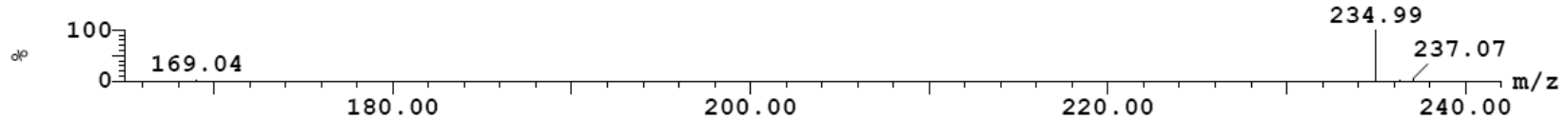




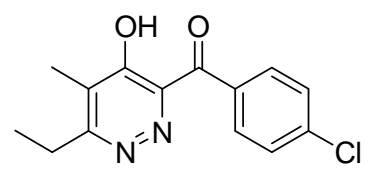

$24 f$

UV Detector: TIC

9. $871 e+1$

Range: $1.148 e+2$

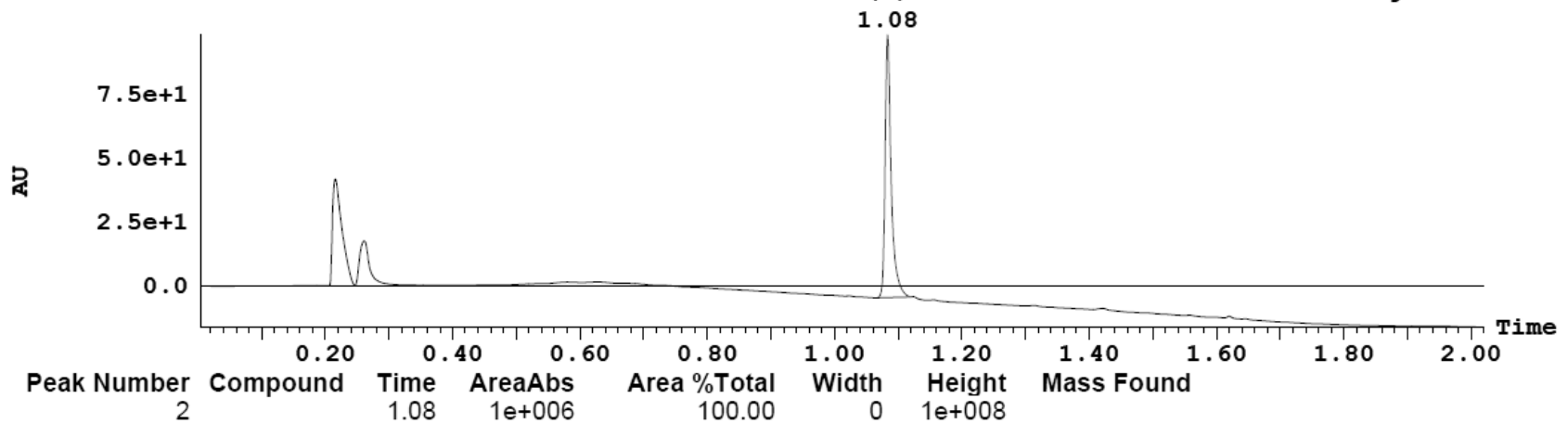

Peak ID Compound Time Mass Found
2

SAMPLE: $1: 20$ Combine (255:259)

$1.8 e+007$

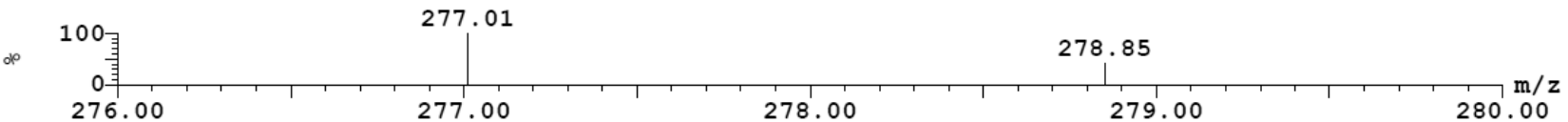<smiles>O=C(c1ccccc1)c1nnc(-c2cccs2)cc1O</smiles>

$24 \mathrm{~g}$

UV Detector: TIC

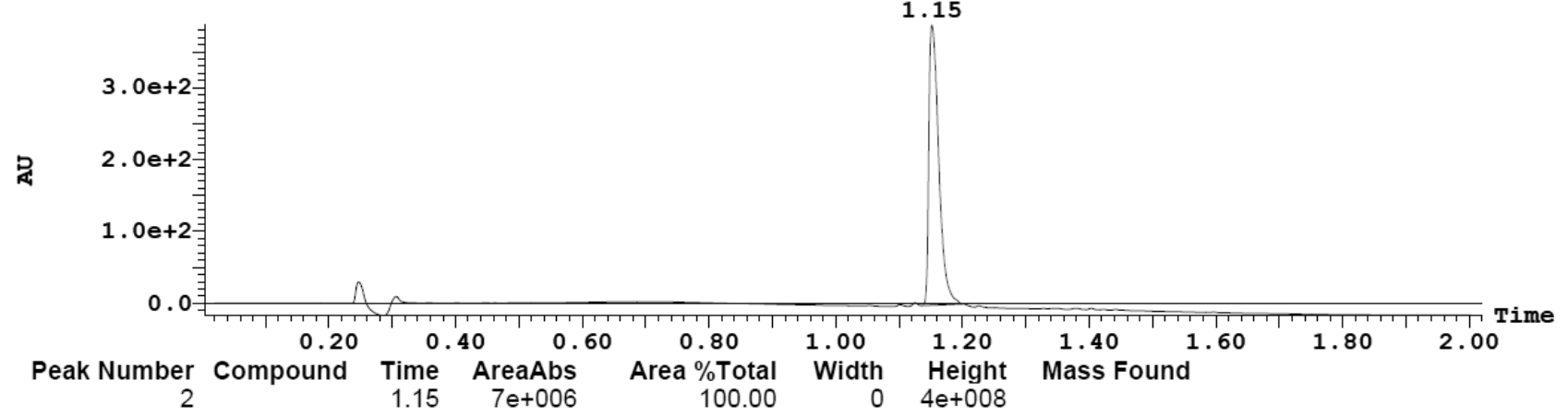

\section{Peak ID Compound Time Mass Found}

SAMPLE: $2: 47$ Combine (271:275)

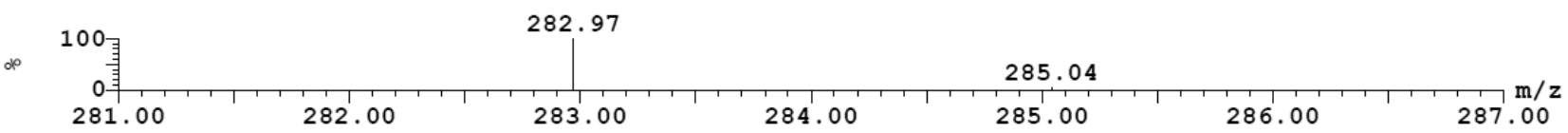


<smiles>O=C(c1ccccc1)c1nnc(-c2ccccc2)cc1O</smiles>

UV Detector: TIC

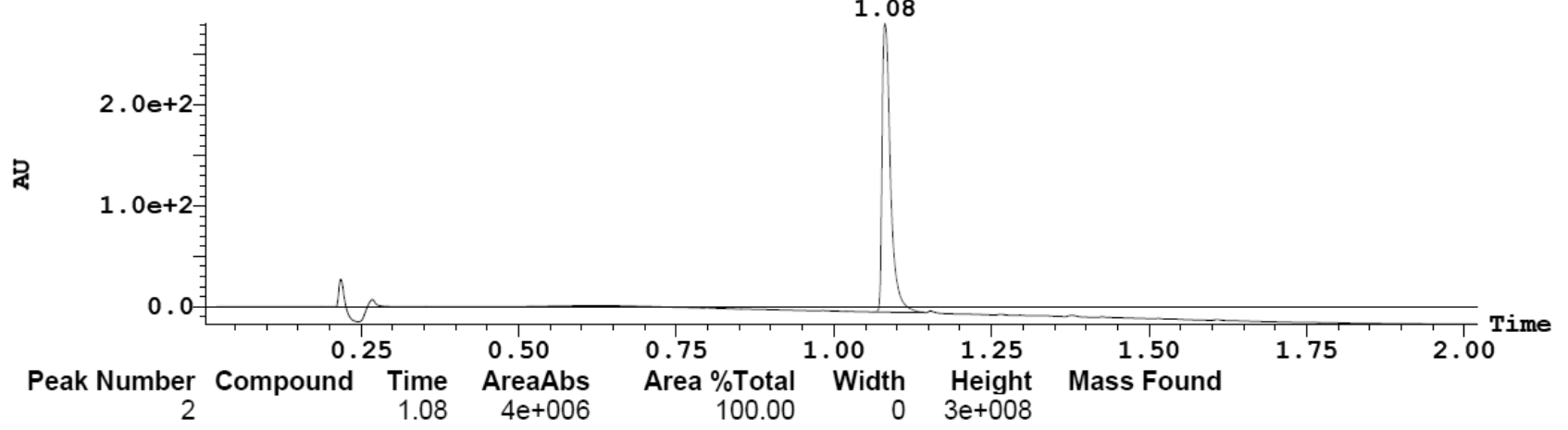

Peak ID Compound Time Mass Found
2

$\begin{array}{lll}\text { SAMPLE: } 2: 13 \text { Combine }(254: 259) & 5.8 e+007\end{array}$

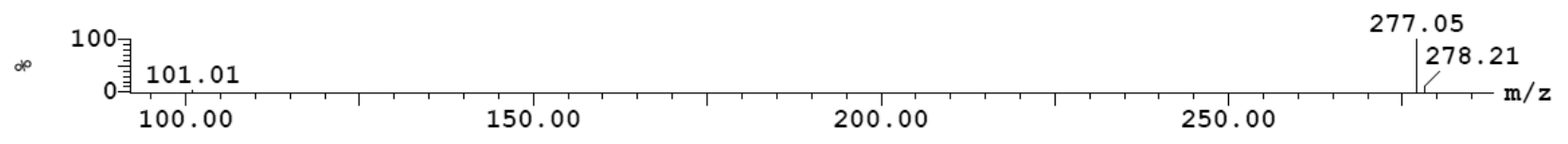<smiles>O=C(c1ccccc1)c1nnc(C2CCCCC2)cc1O</smiles>

UV Detector: TIC

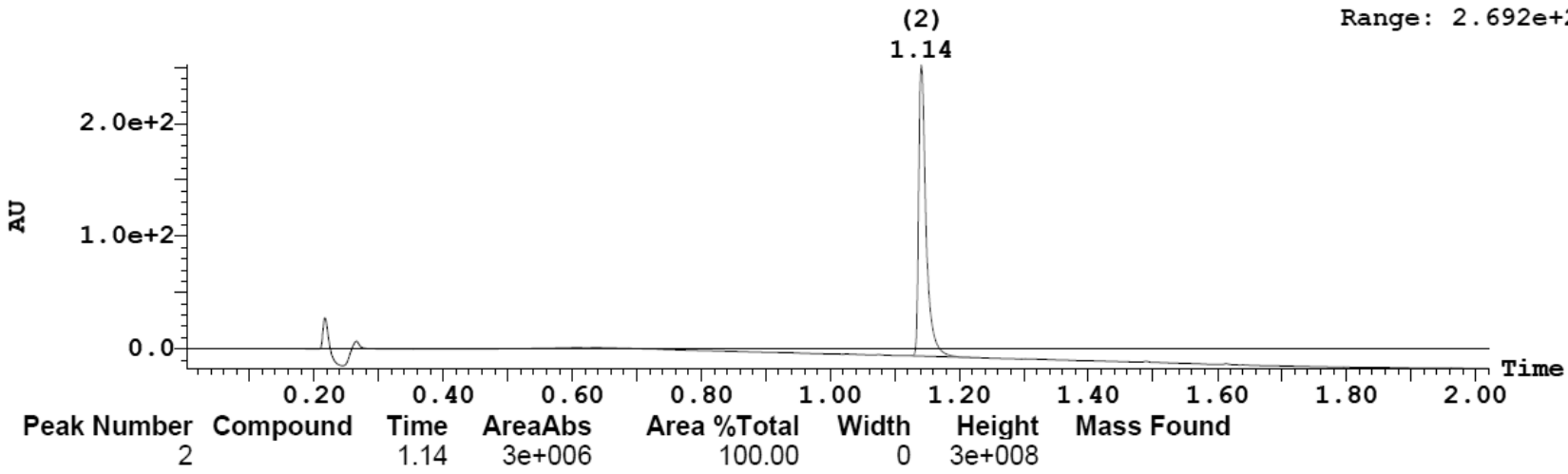

\section{Peak ID Compound Time Mass Found}

SAMPLE: $2: 14$ Combine $(268: 273)$

8. $9 e+007$

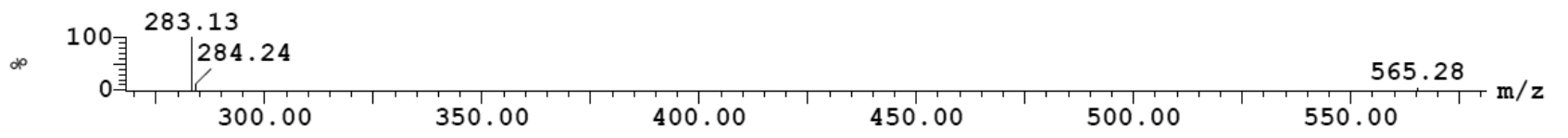




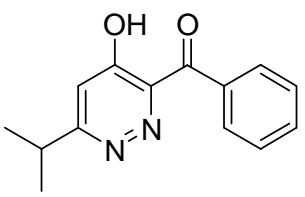

$24 j$

UV Detector: TIC

(2)

0.96
$3.33 e+2$

Range: $3.499 e+2$

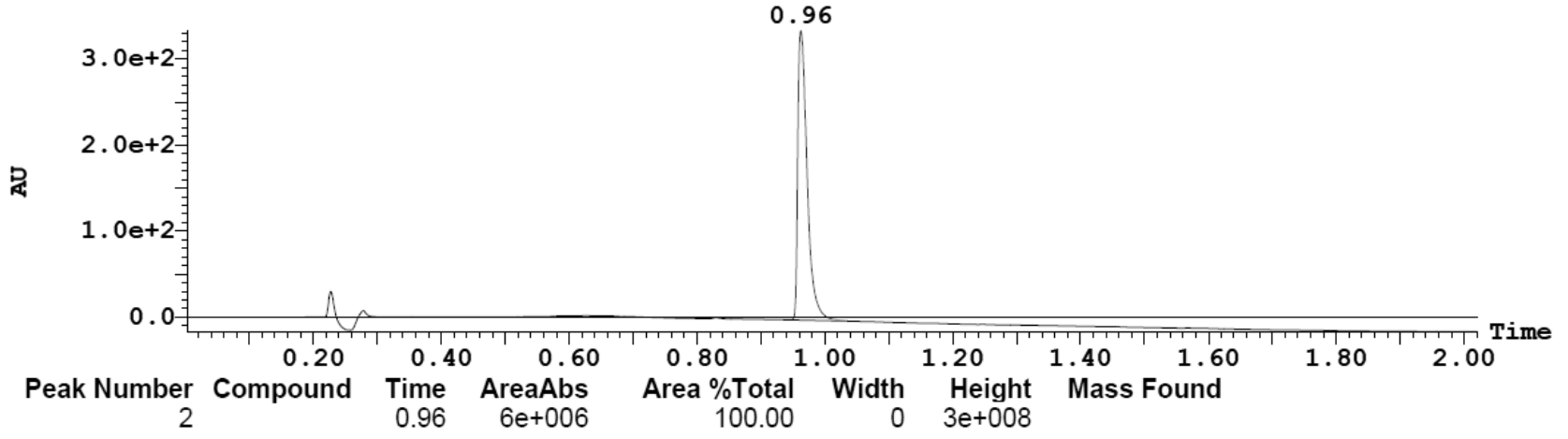

Peak ID Compound Time
0.96

SAMPLE: $1: 37$ Combine $(226: 231)$

$1.1 e+008$

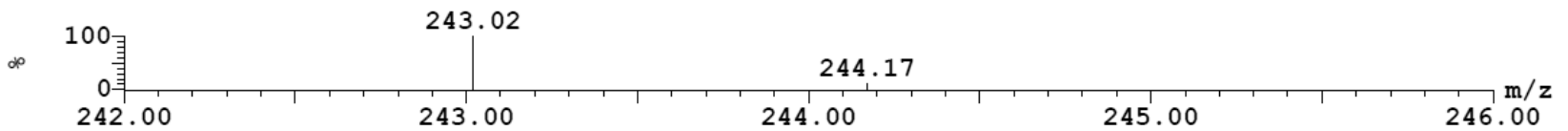




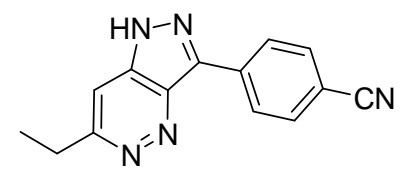

$25 a$

UV Detector: TIC

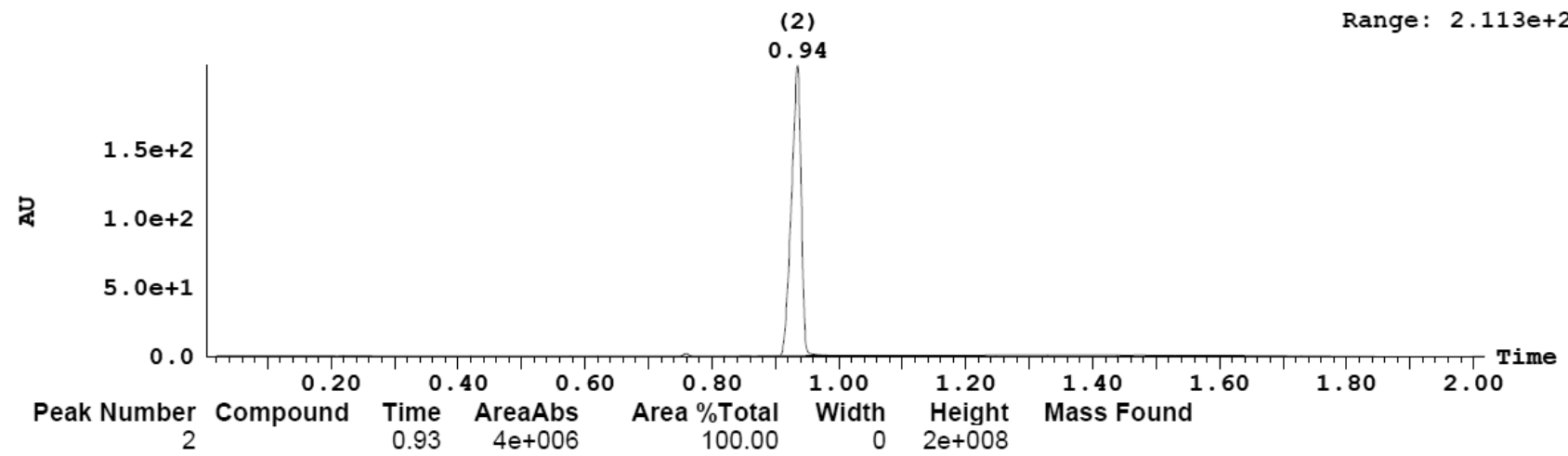

Peak ID Compound Time Mass Found

2
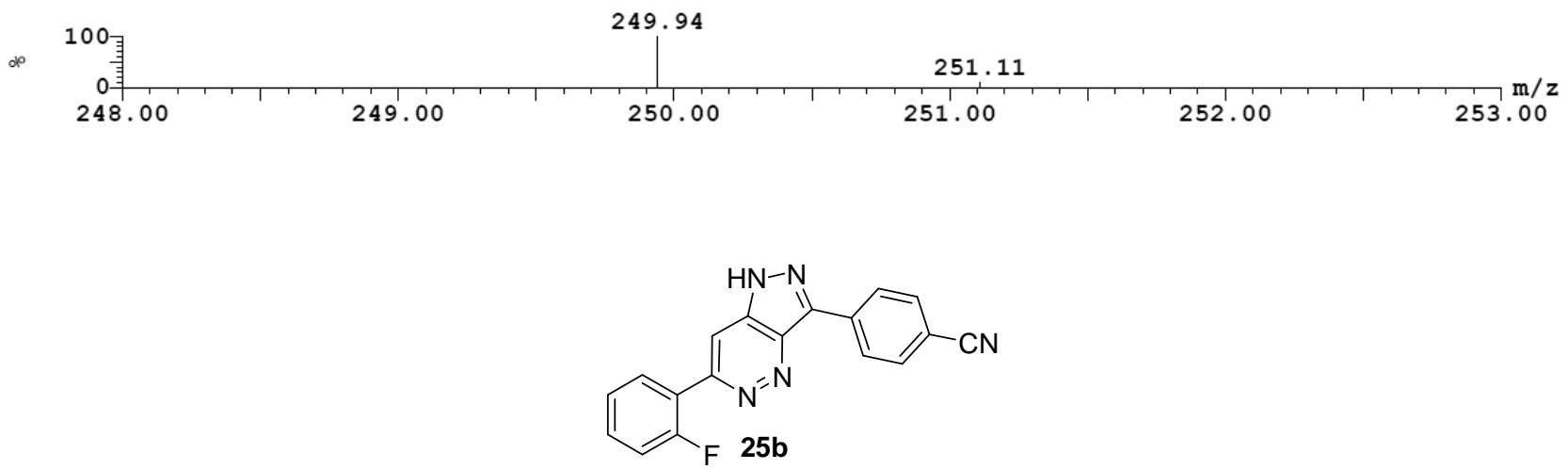

UV Detector: TIC
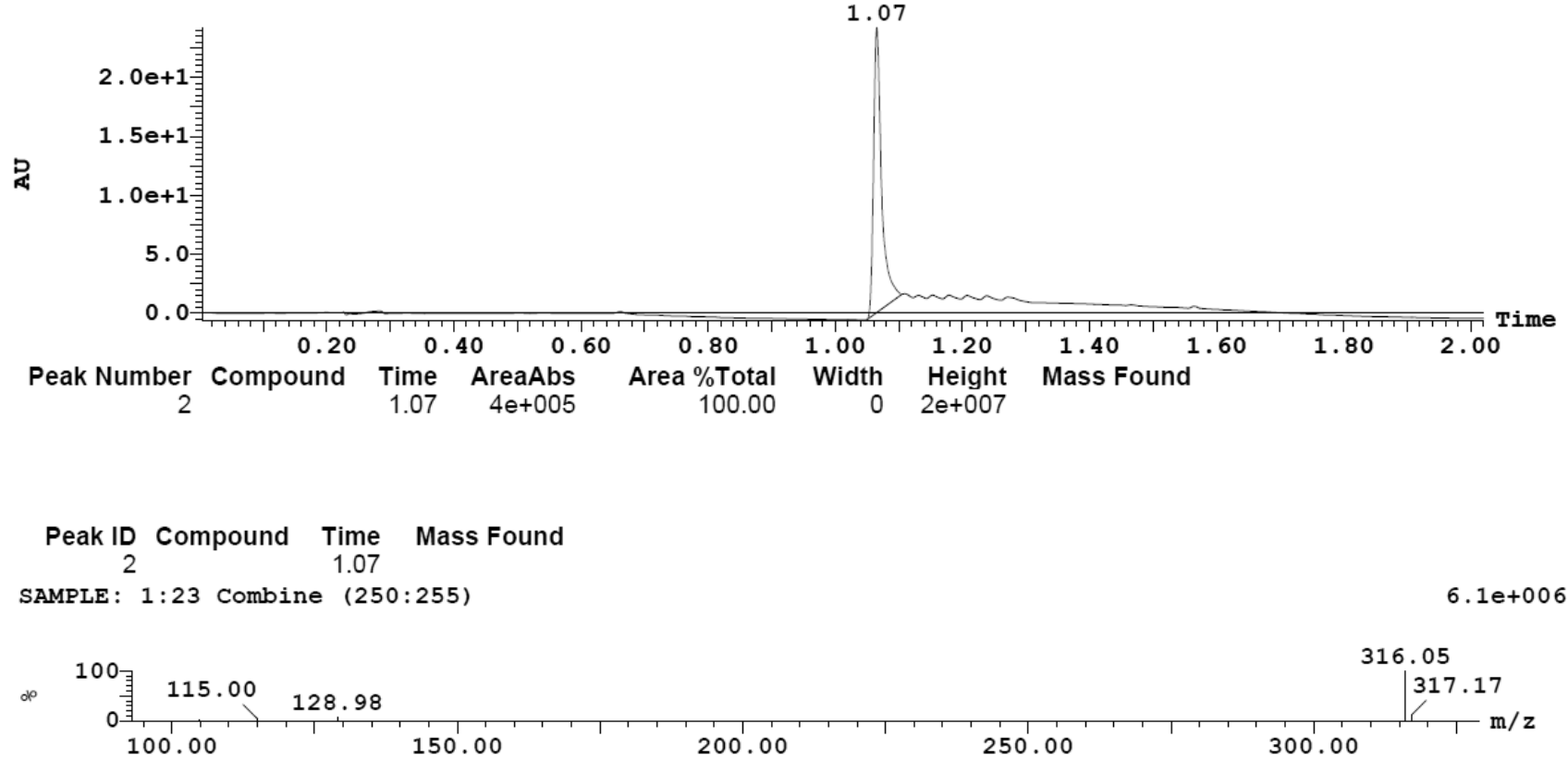


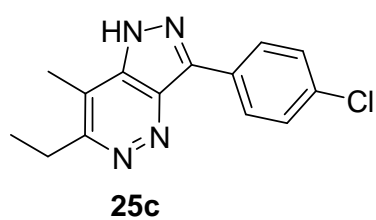

UV Detector: TIC

8. $962 e+1$

(1)

Range: $8.962 e+1$

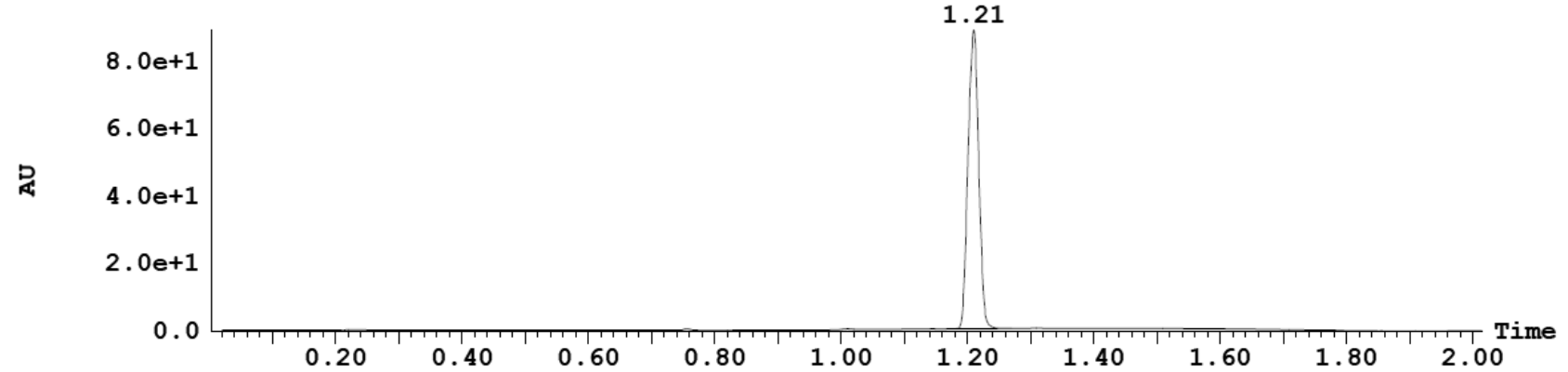

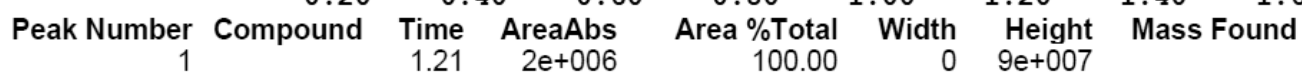

$1.212 e+006$

\section{Peak ID Compound Time Mass Found
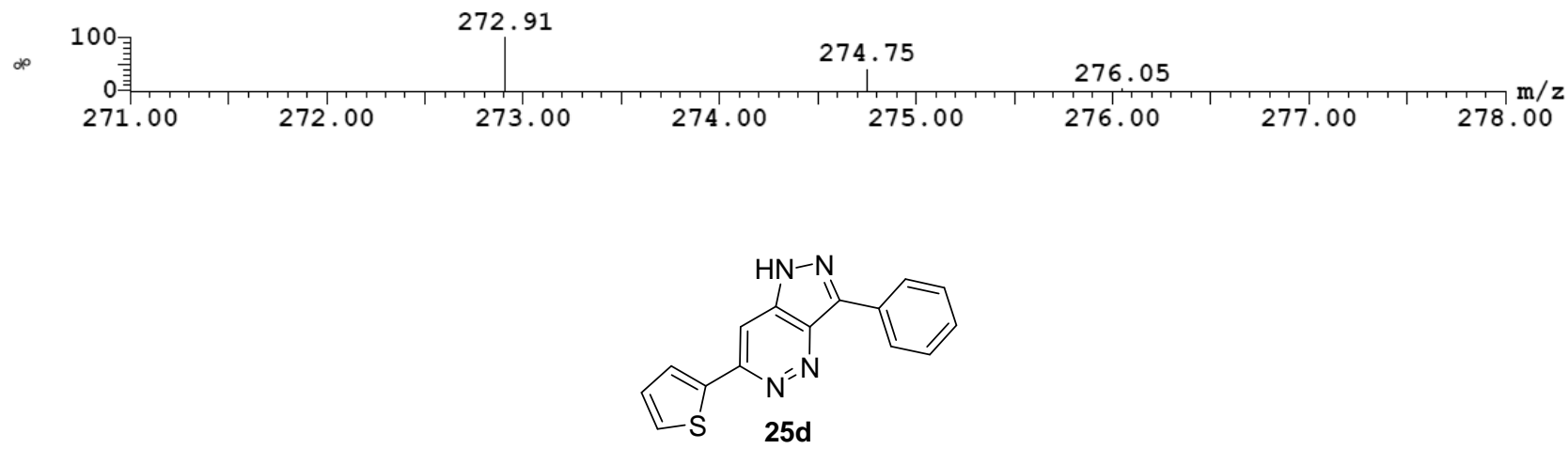

UV Detector: TIC

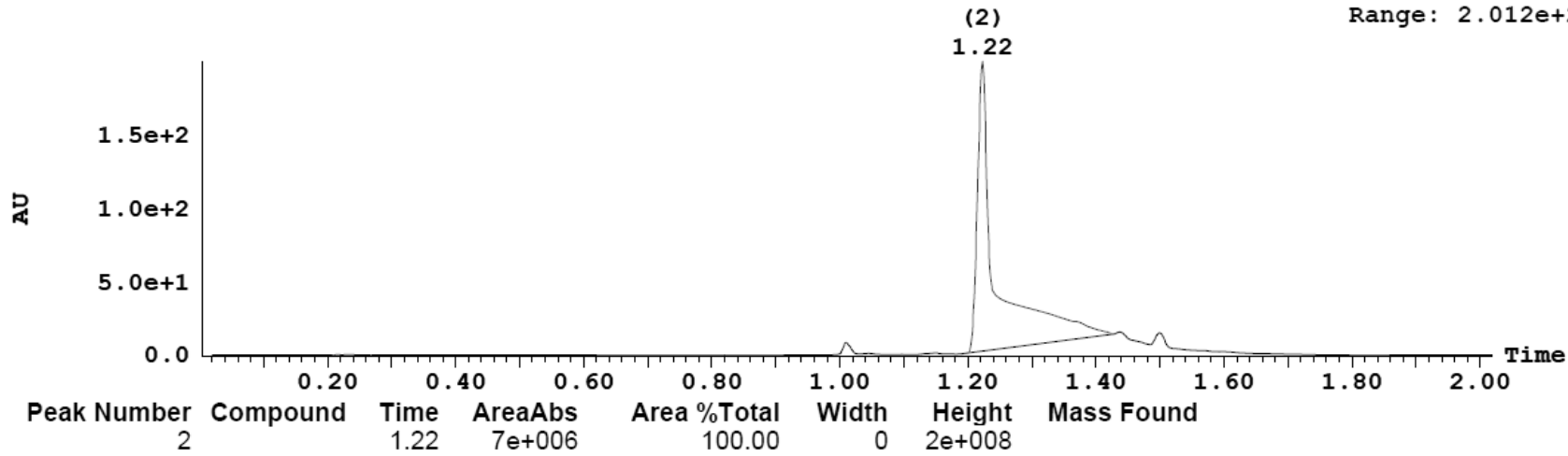

\section{Peak ID Compound Time Mass Found
2}

SAMPLE: 2:15 Combine (289:293)

$1 \cdot 7 e+007$

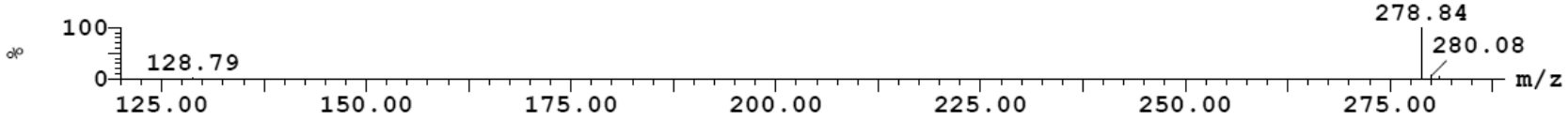




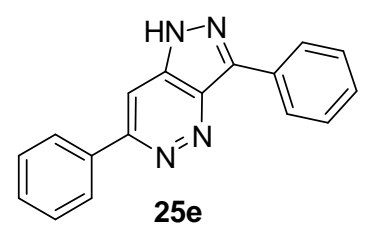

UV Detector: TIC

$7.906 e+1$

Range: $7.906 e+1$

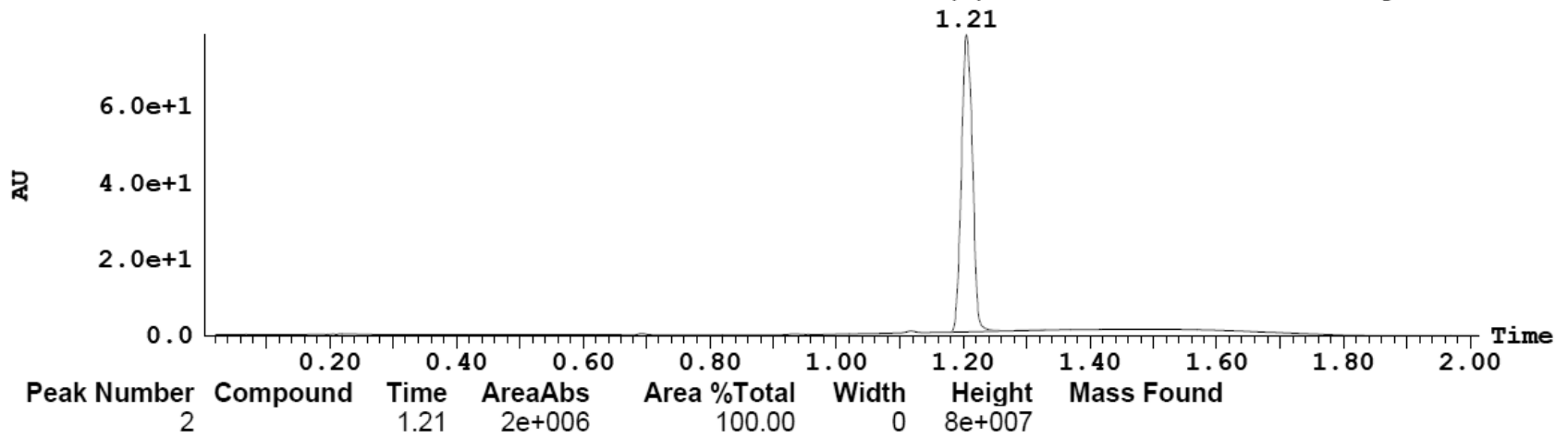

\section{Peak ID Compound Time Mass Found

2 1.21

SAMPLE: $2: 13$ Combine $(284: 289)$

$1.7 e+007$

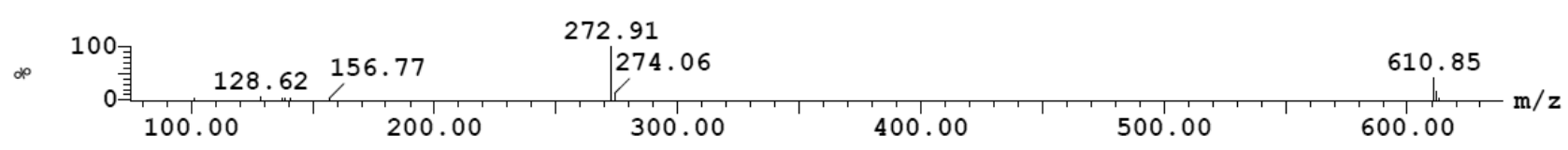<smiles>CC(C)c1cc2[nH]nc(-c3ccccc3)c2nn1</smiles>

$25 f$

UV Detector: TIC

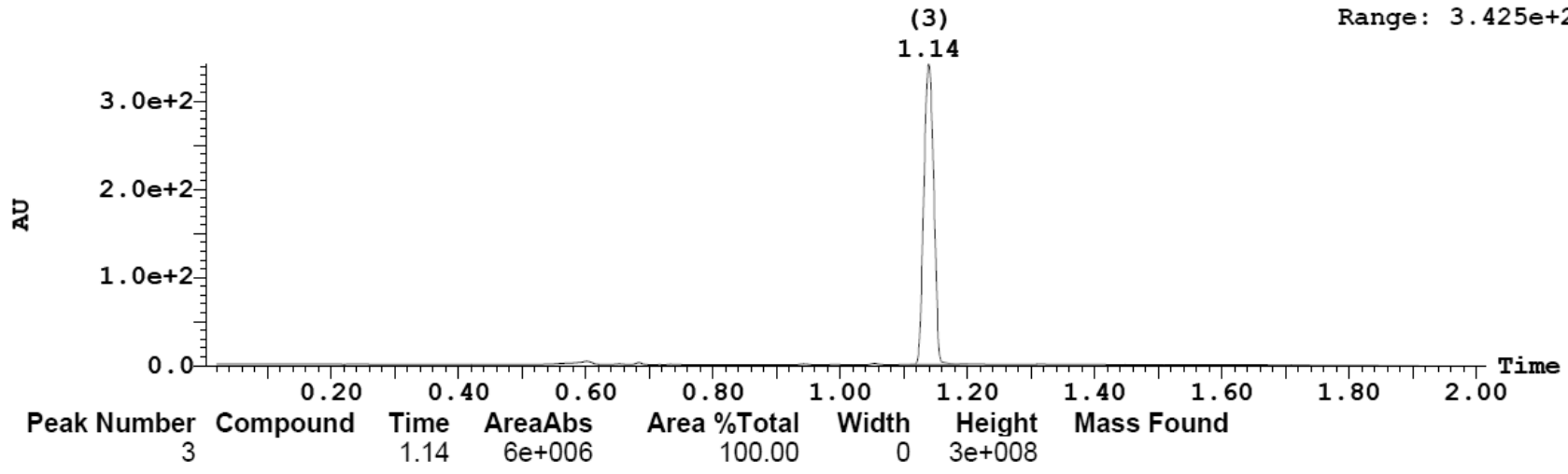

\section{Peak ID Compound Time Mass Found}

SAMPLE: $2: 48$ Combine $(269: 273)$

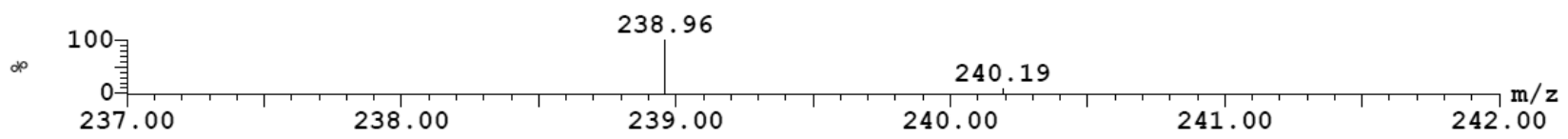




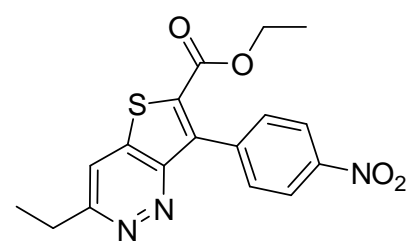

26a

UV Detector: TIC

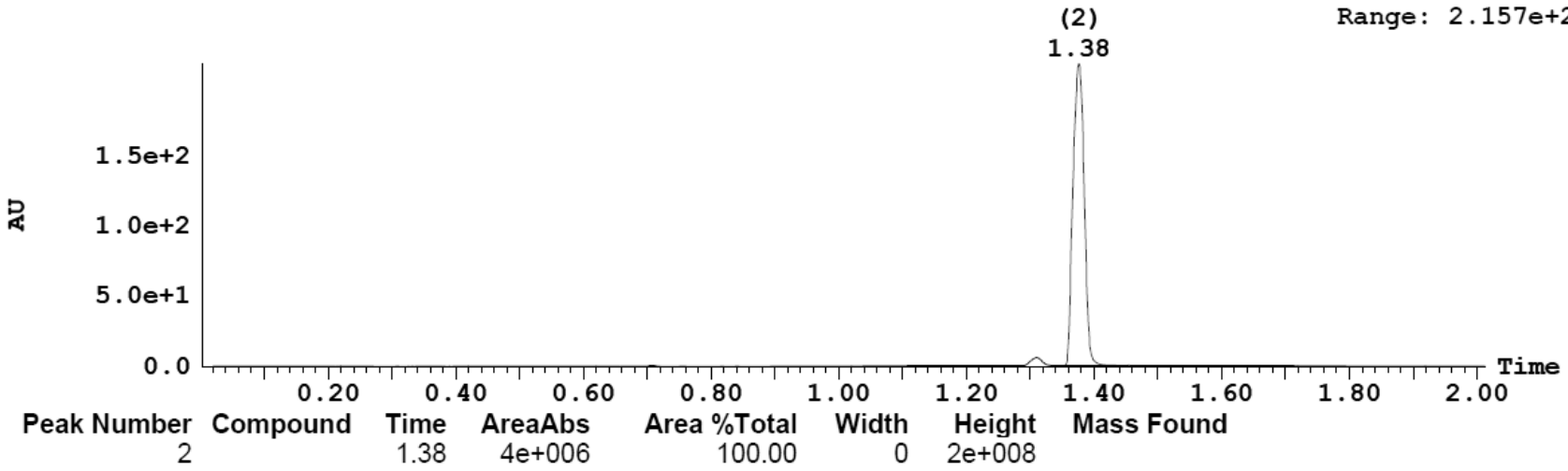

\section{Peak ID Compound Time
1.38}

SAMPLE: 2:21 Combine (325:330)
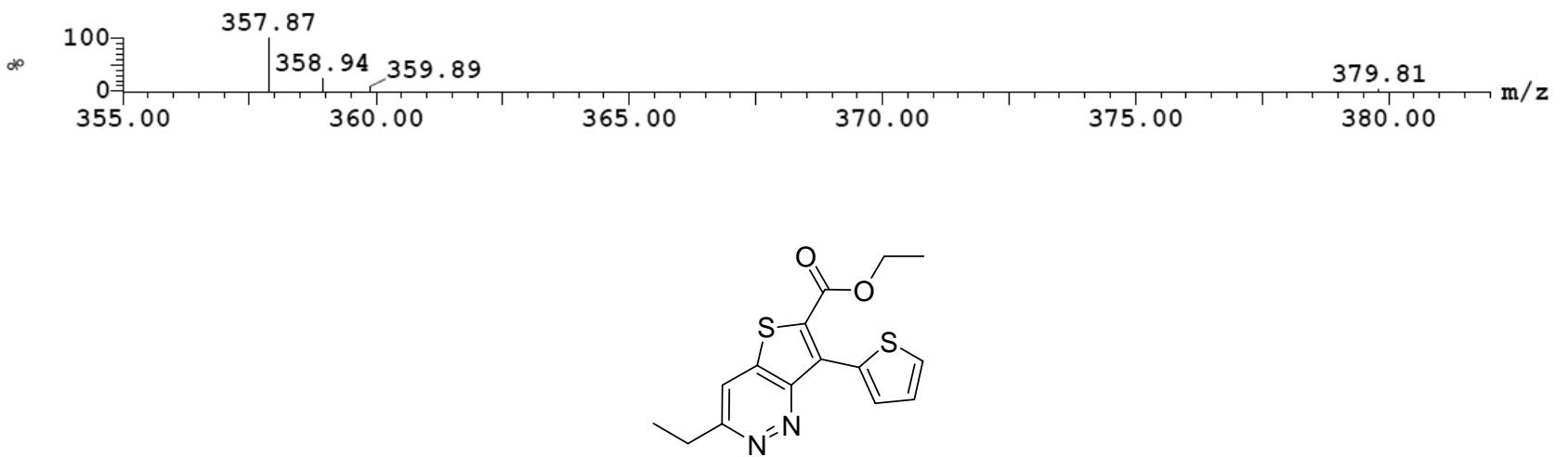

26b

UV Detector: TIC

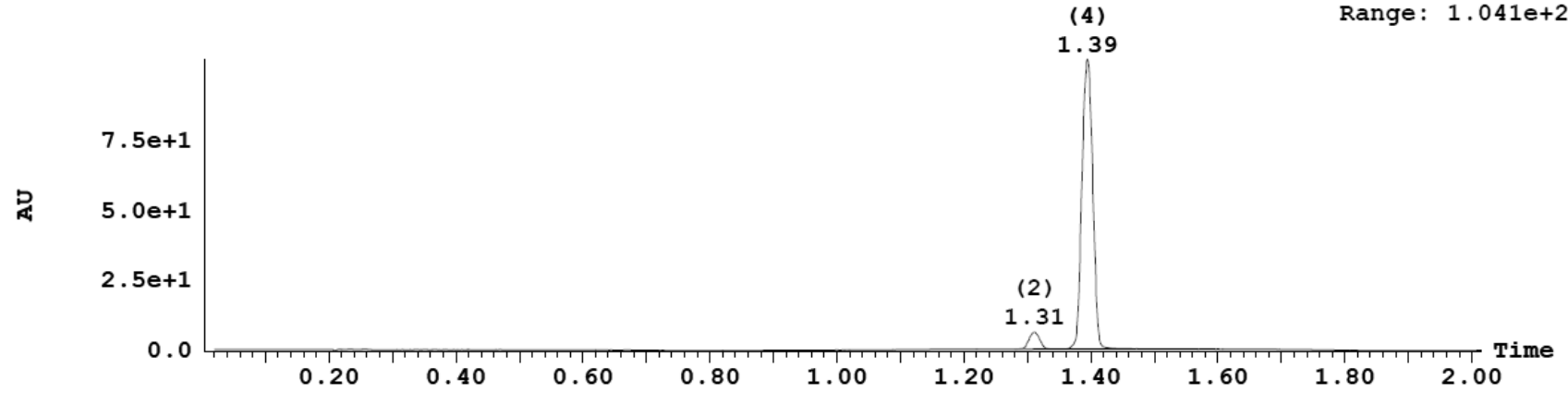

Peak Number Compound Time AreaAbs

$\begin{array}{rrrrrr}2 & 1.31 & 1 e+005 & 5.42 & 0 & 6 e+006 \\ 4 & 1.39 & 2 e+006 & 94.58 & 0 & 1 e+008\end{array}$

Area \%Total Width Height

Mass Found

Peak ID Compound Time
4

SAMPLE: $1: 6$ Combine (329:334)

1. $1 e+008$

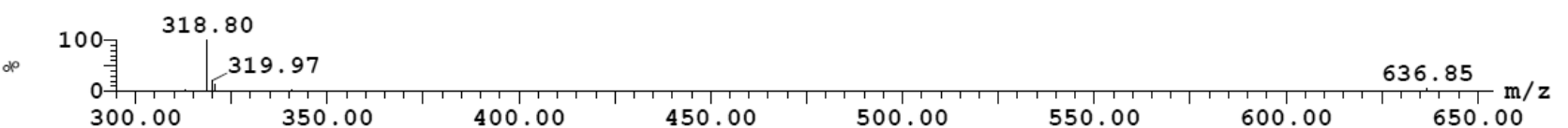




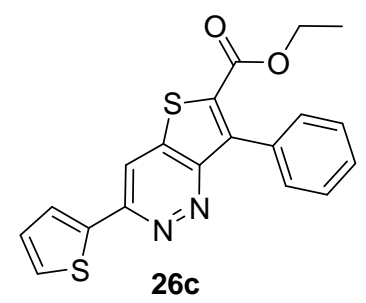

UV Detector: TIC

2. $517 e+2$
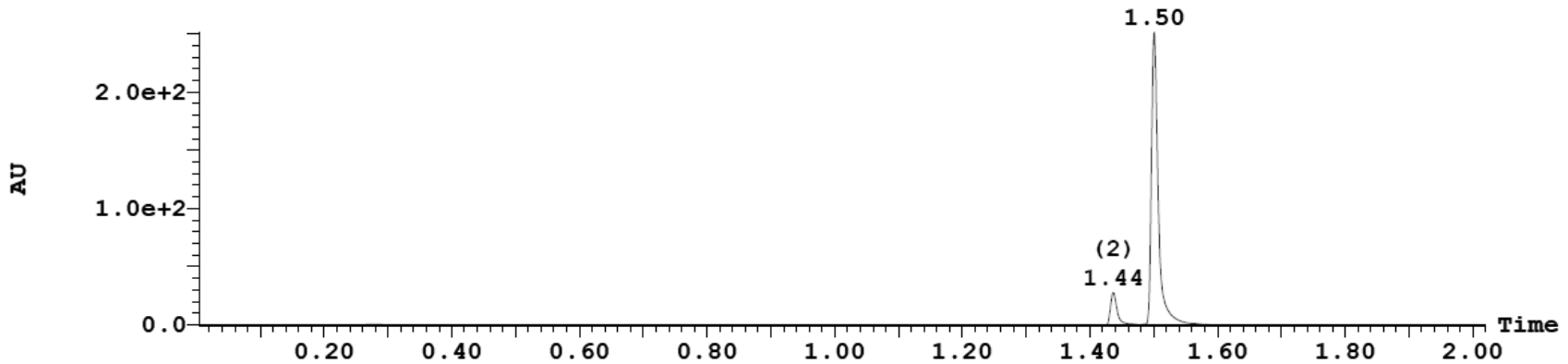

Peak Number Compound 2 $\begin{array}{rr}\text { Time } & \text { AreaAbs } \\ 1.44 & 4 e+005 \\ 1.50 & 3 e+006\end{array}$ Area \%Total
9.97
90.03

$90.03 \quad 0 \quad 3 e+008$

\section{Peak ID Compound Time Mass Found}

SAMPLE: $1: 12$ Combine (354:358)

$2.7 e+007$
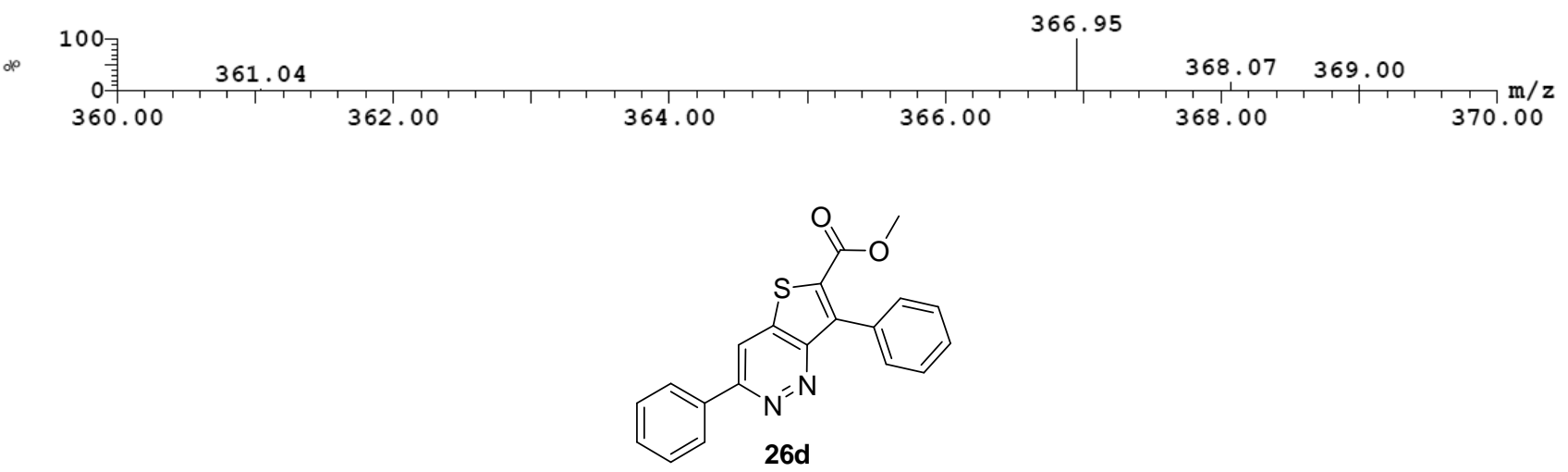

UV Detector: TIC

$1.776 \mathrm{e}+2$

Range: $1.776 \mathrm{e}+2$

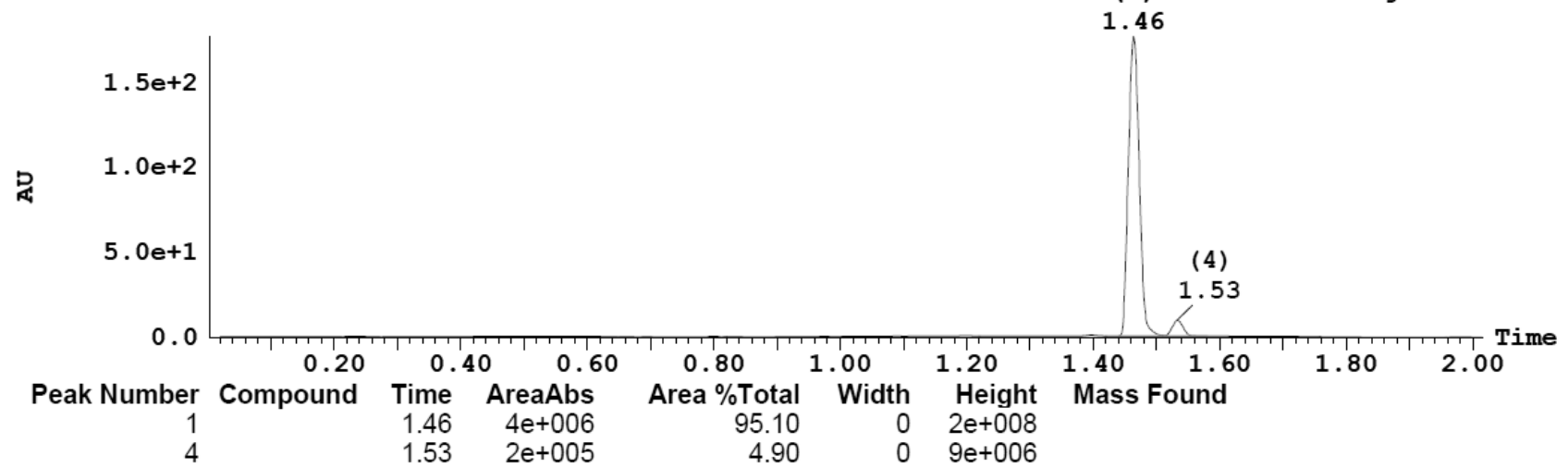

\section{Peak ID Compound Time Mass Found}

SAMPLE: 2:15 Combine (346:351)

$6.9 e+007$

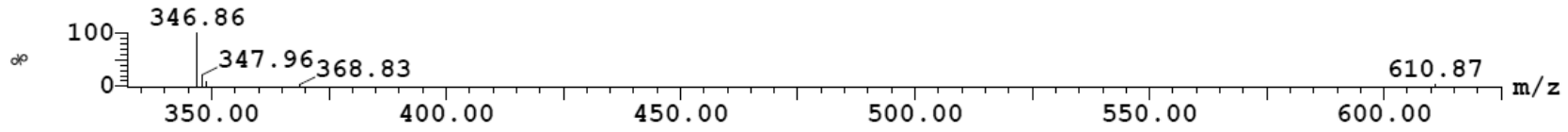




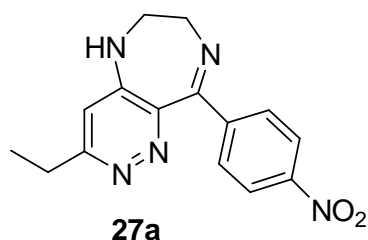

UV Detector: TIC

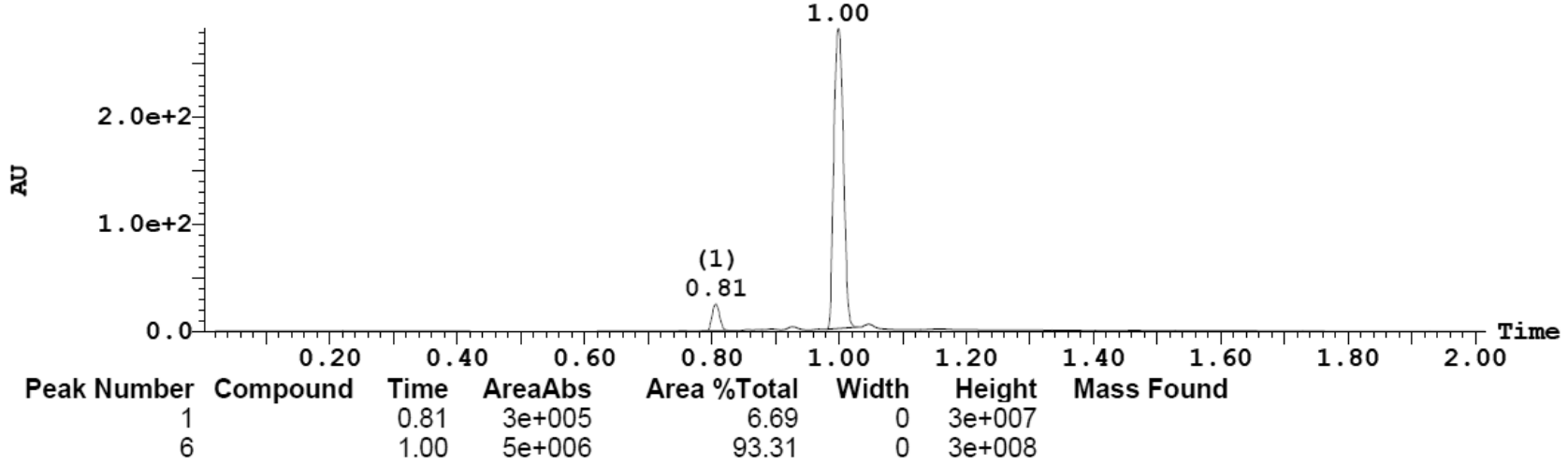

\section{Peak ID Compound Time Mass Found}

SAMPLE : 2:16 Combine (235:240)
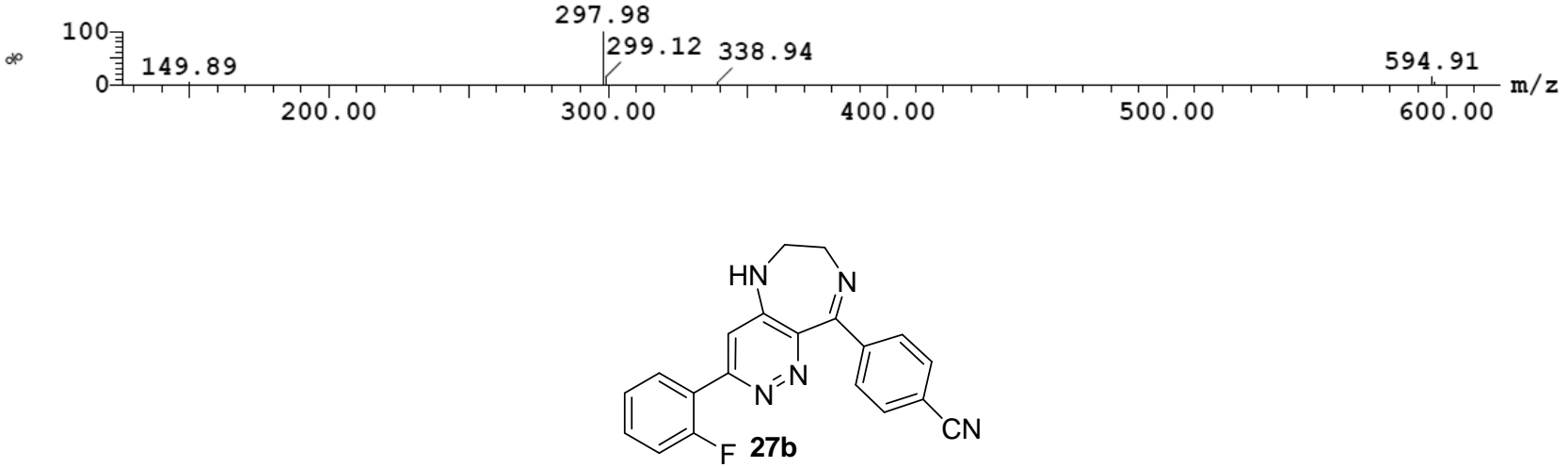

UV Detector: TIC

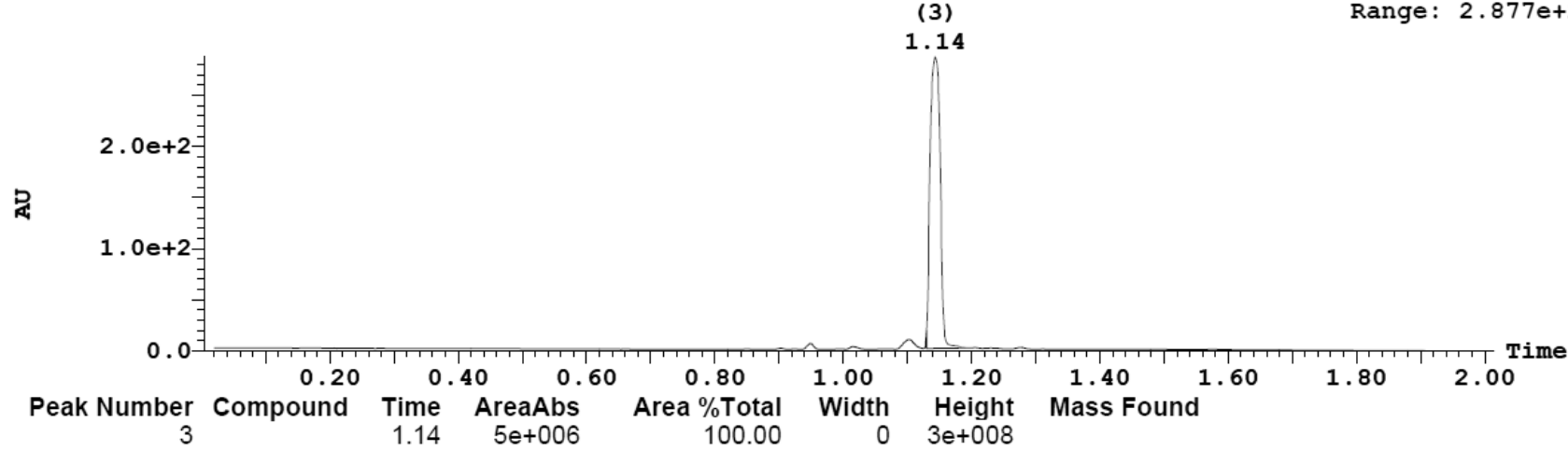

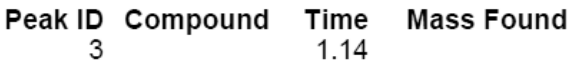

SAMPLE: 2:11 Combine (270:274)

$7.1 e+007$

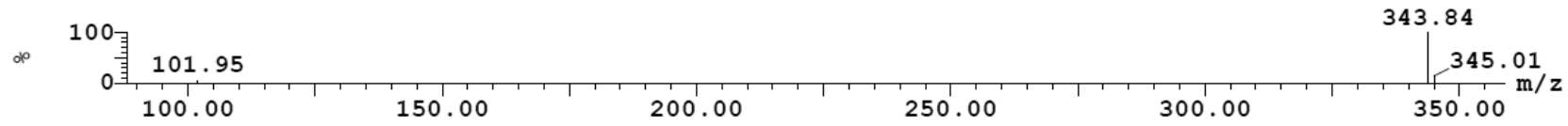




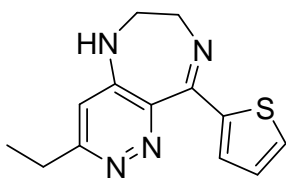

$27 c$

UV Detector: TIC

(4)

$2.596 e+2$

Range: $2.596 \mathrm{e}+2$

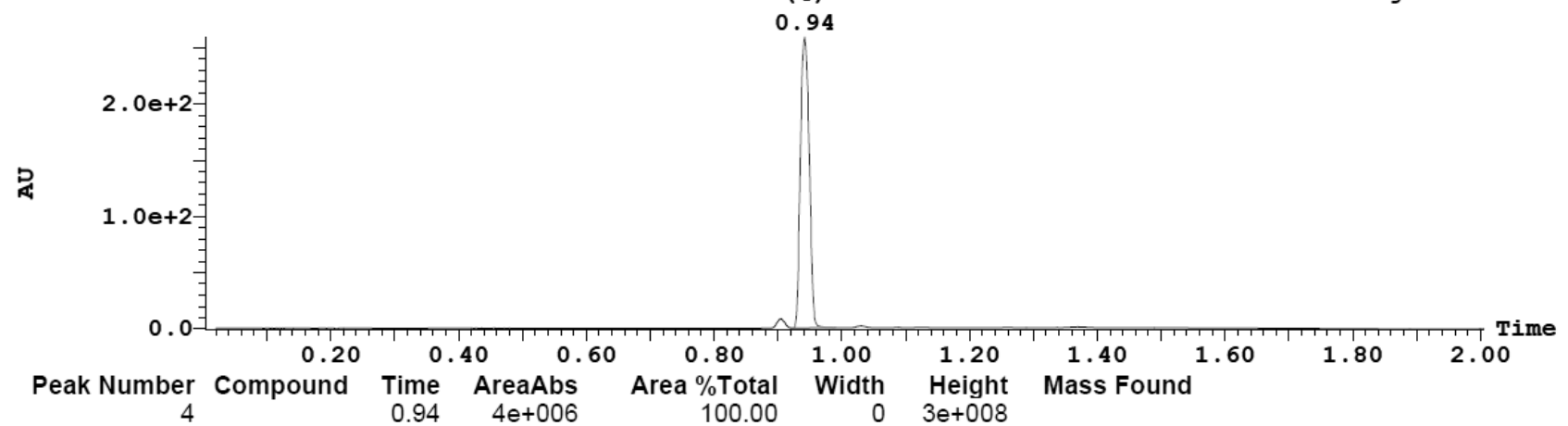

Peak ID Compound Time Mass Found

SAMPLE: $1: 13$ Combine (222:226)

1. $3 e+008$
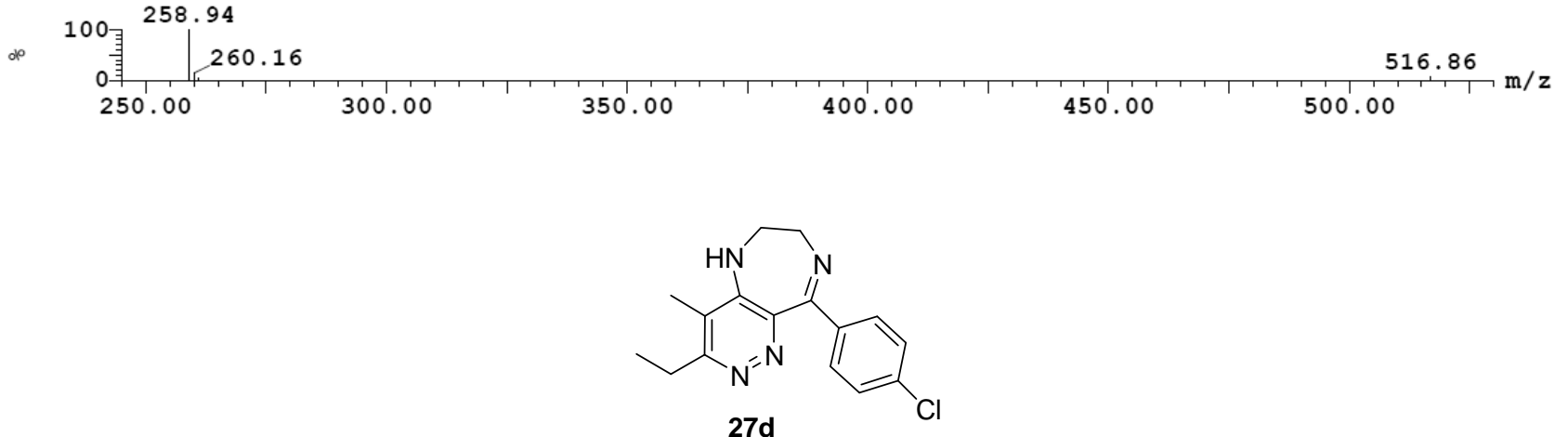

UV Detector: TIC

(1)

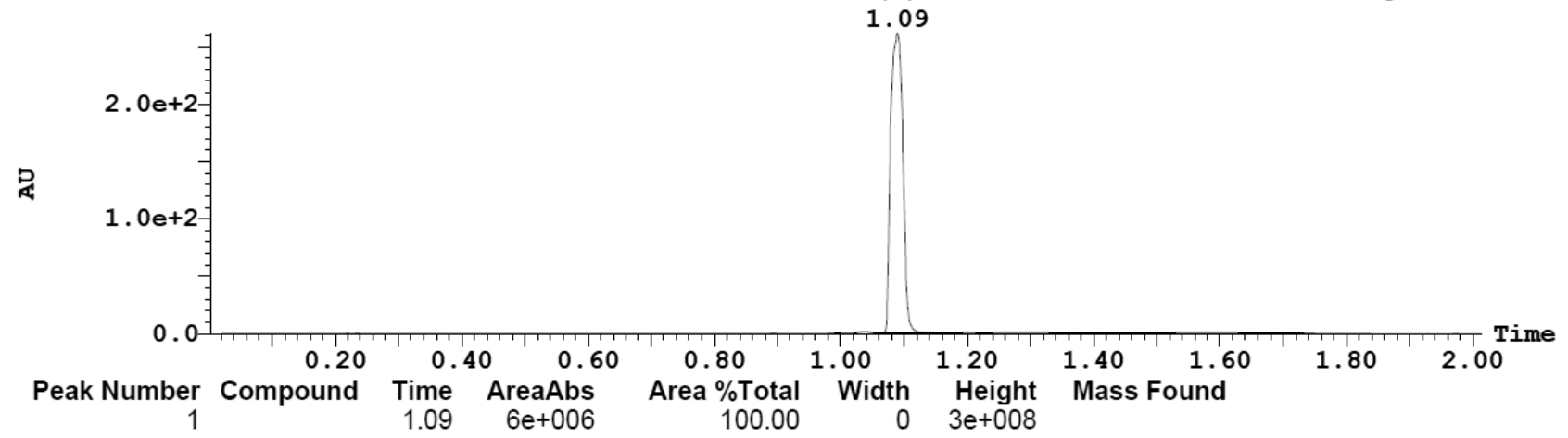

\section{Peak ID Compound Time Mass Found \\ 11.09}

SAMPLE: 2:8 Combine (257:262)

1. $1 e+008$

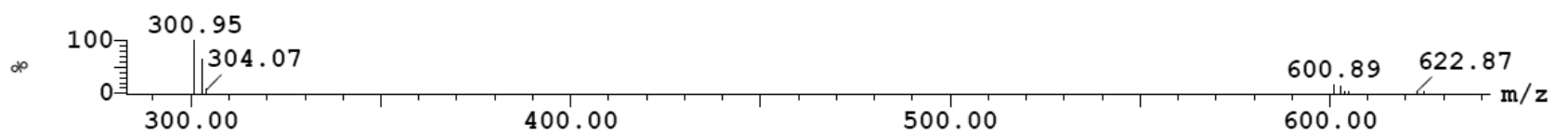




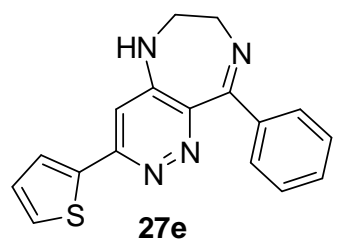

UV Detector: TIC

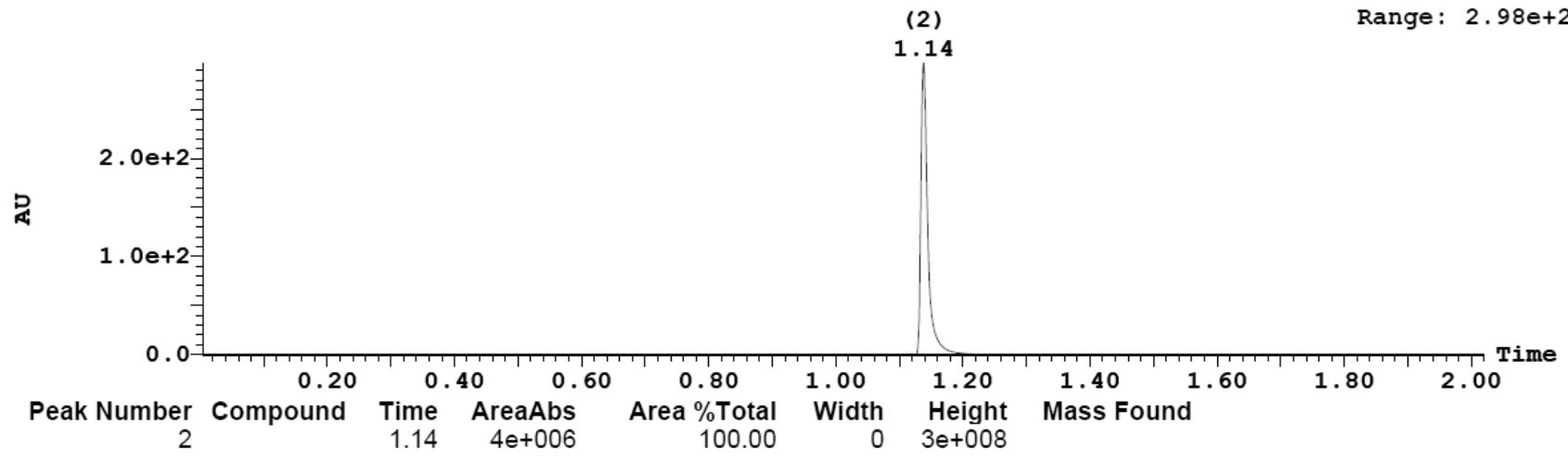

Peak ID Compound Time Mass Found

SAMPLE: $1: 24$ Combine $(268: 272)$

$3.7 e+007$
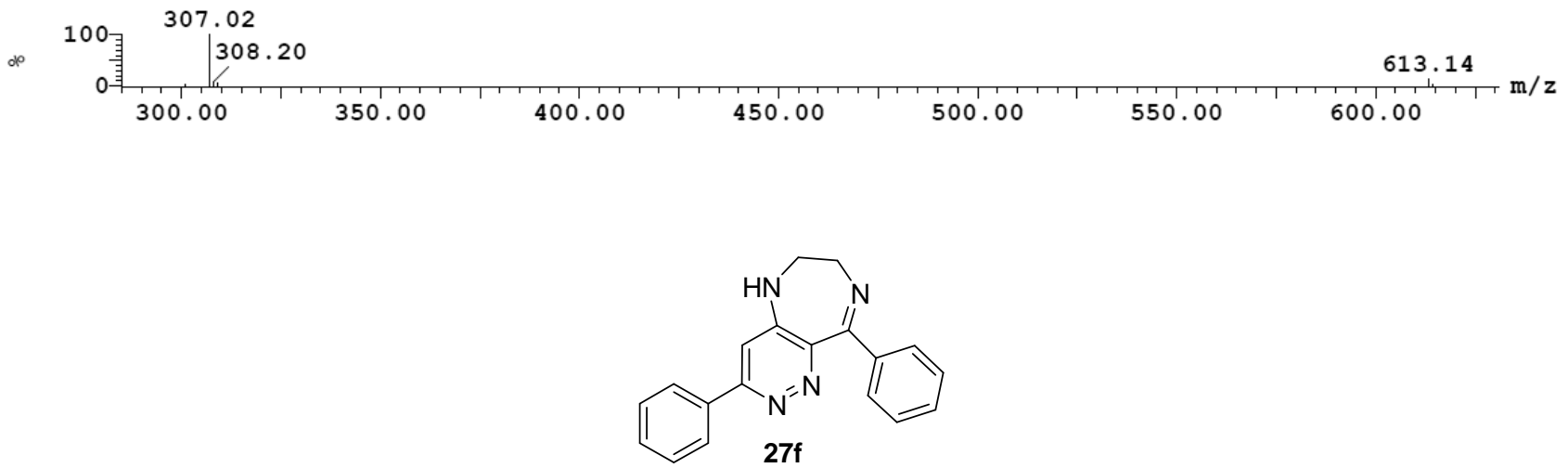

UV Detector: TIC

(1)

$2.153 e+2$

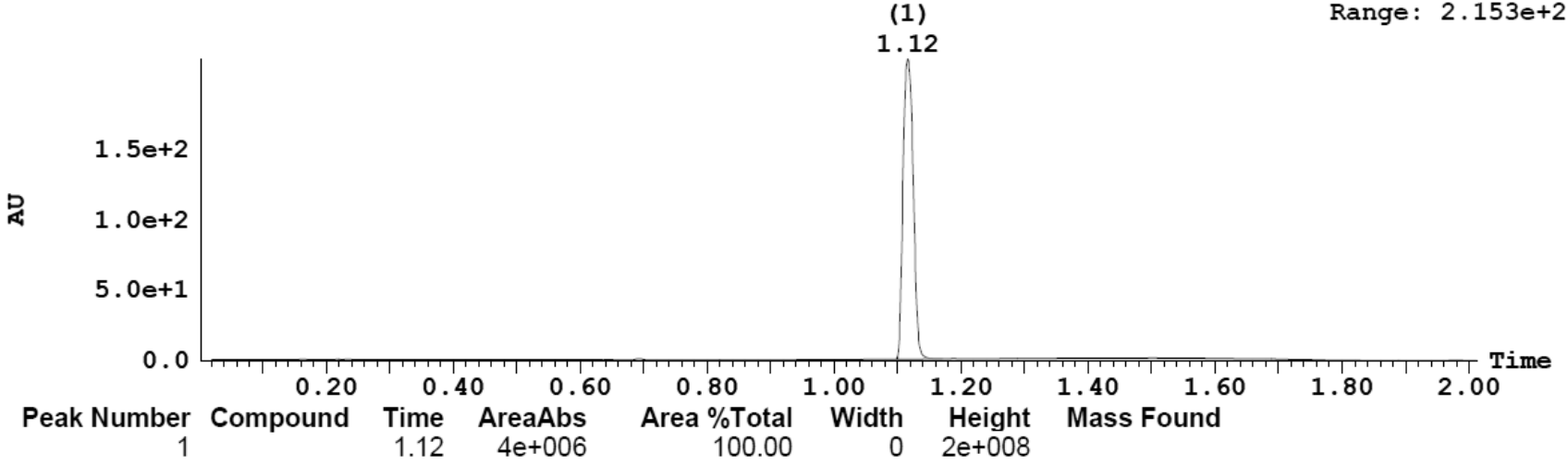

Peak ID Compound Time Mass Found 
<smiles>c1ccc(C2=NCCNc3cc(C4CCCCC4)nnc32)cc1</smiles>

UV Detector: TIC

$1.889 e+2$

$(2)$

Range: $1.889 e+2$

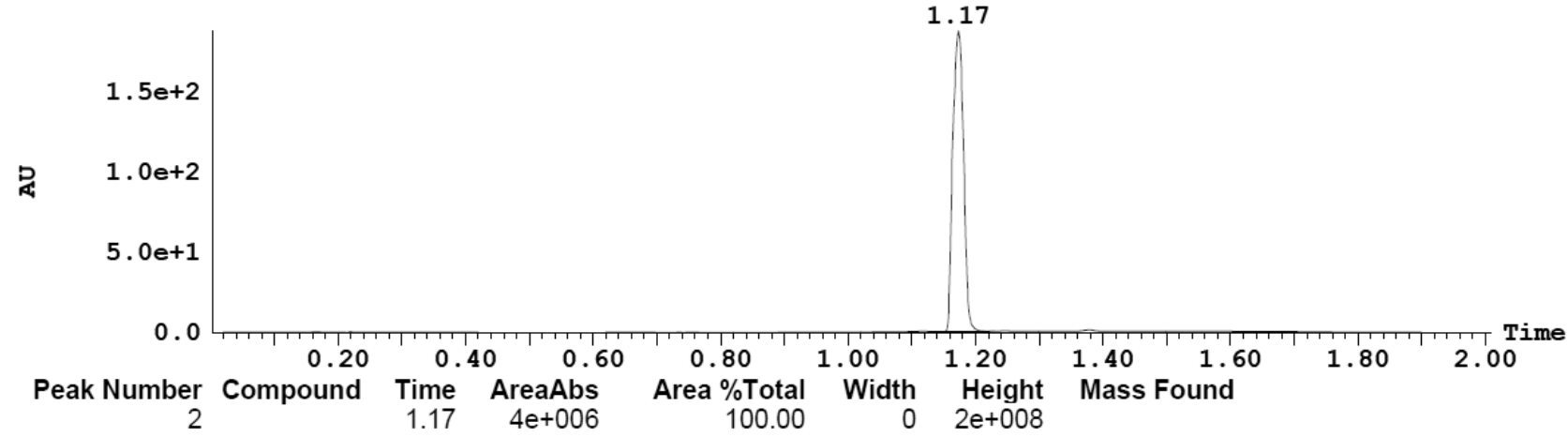

Peak ID Compound Time
1.17

SAMPLE: $2: 22$ Combine $(277: 282) \quad 1.1 e+008$
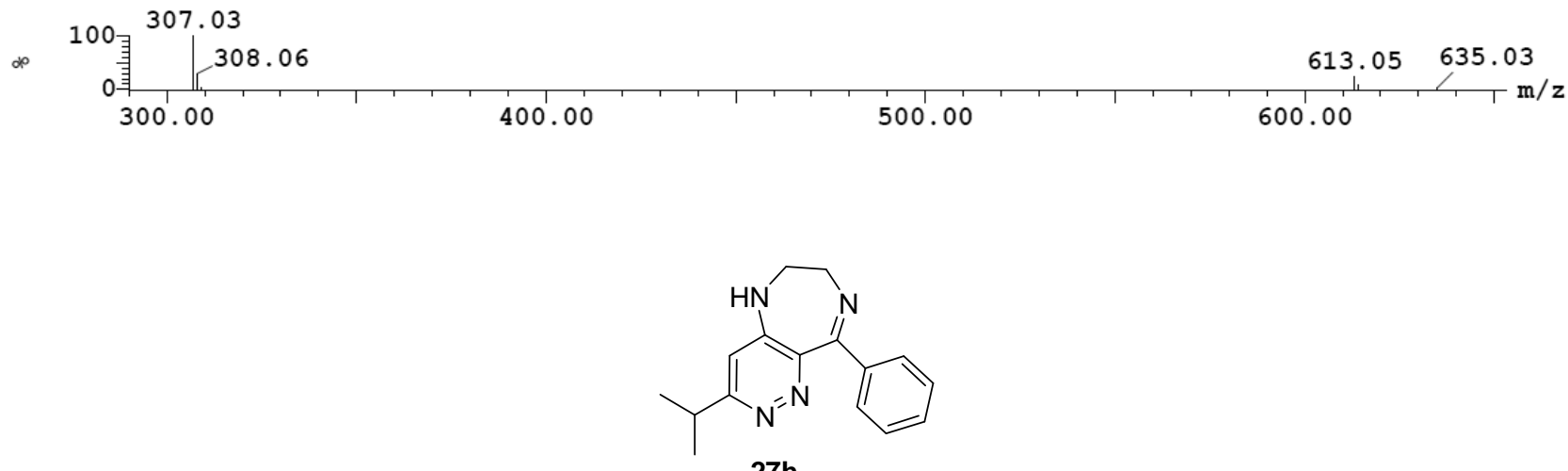

27h

UV Detector: TIC

(2)

$3.36 e+2$

UV Detector: IIC

Range: $3.36 e+2$

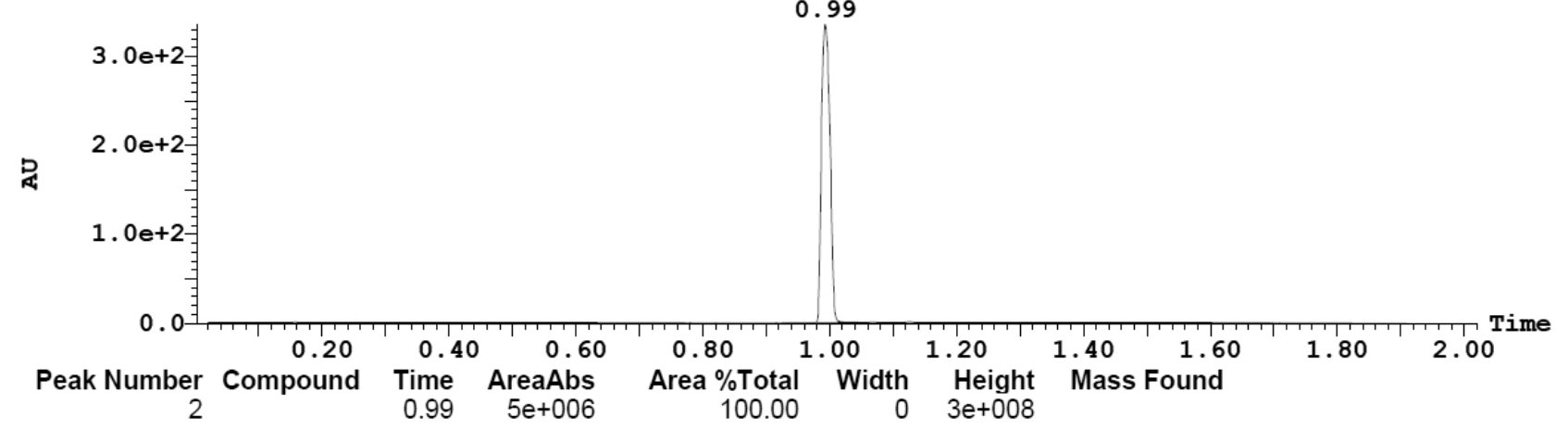

\section{Peak ID Compound Time Mass Found}

SAMPLE: $1: 2$ Combine (234:239)

1. $3 e+008$

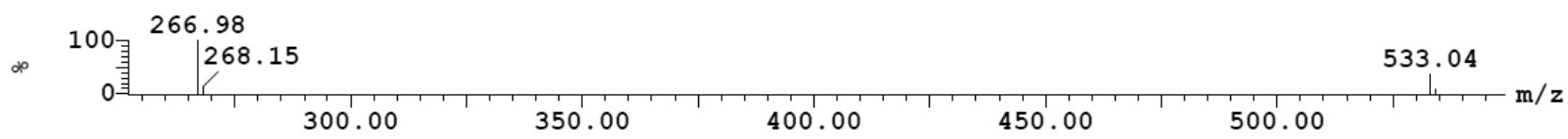




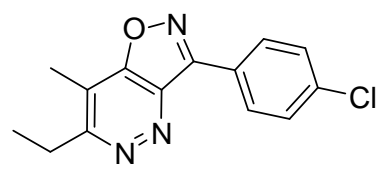

$28 a$

UV Detector: TIC

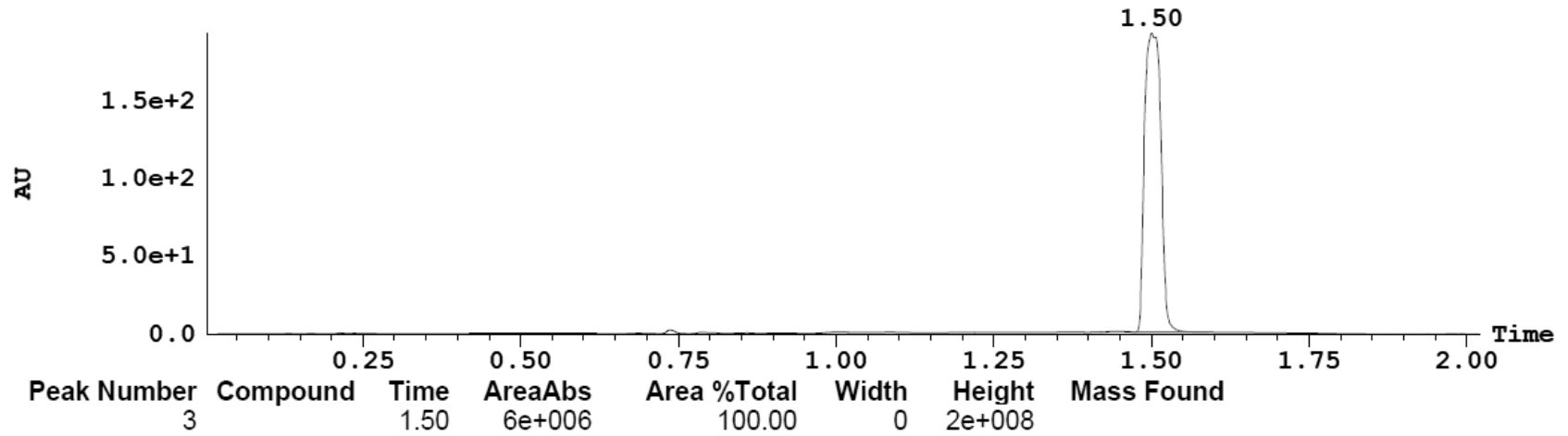

\section{Peak ID Compound Time Mass Found
3}

SAMPLE: $2: 9$ Combine (355:359)
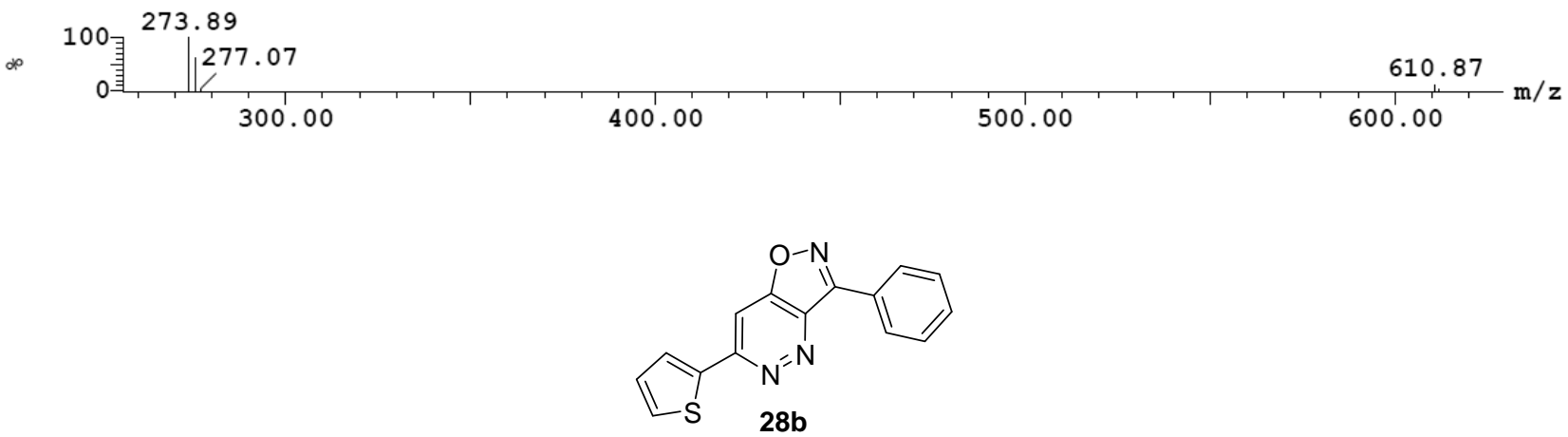

UV Detector: TIC

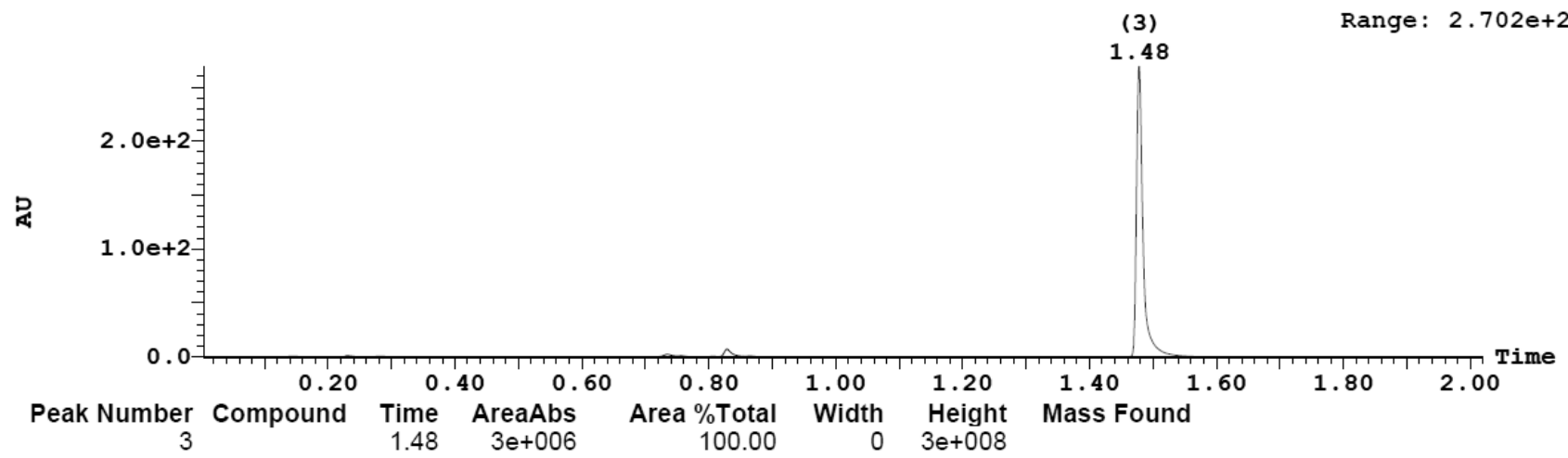

\section{Peak ID Compound Time
3}

SAMPLE: $1: 13$ Combine $(348: 353)$

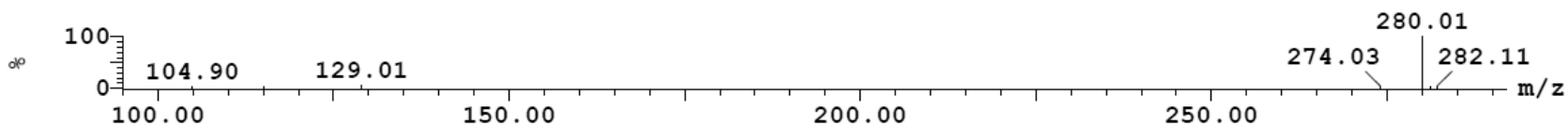

